\title{
Dual practice of medical professionals in public hospitals in south- eastern Nigeria: An economic and policy analysis
}

\author{
Eze, Bartholomew S.
}

How to cite:

Eze, Bartholomew S. (2019) Dual practice of medical professionals in public hospitals in south-eastern Nigeria: An economic and policy analysis. Doctoral thesis, Swansea University.

http://cronfa.swan.ac.uk/Record/cronfa51641

Use policy:

This item is brought to you by Swansea University. Any person downloading material is agreeing to abide by the terms of the repository licence: copies of full text items may be used or reproduced in any format or medium, without prior permission for personal research or study, educational or non-commercial purposes only. The copyright for any work remains with the original author unless otherwise specified. The full-text must not be sold in any format or medium without the formal permission of the copyright holder. Permission for multiple reproductions should be obtained from the original author.

Authors are personally responsible for adhering to copyright and publisher restrictions when uploading content to the repository.

Please link to the metadata record in the Swansea University repository, Cronfa (link given in the citation reference above.)

http://www.swansea.ac.uk/library/researchsupport/ris-support/ 

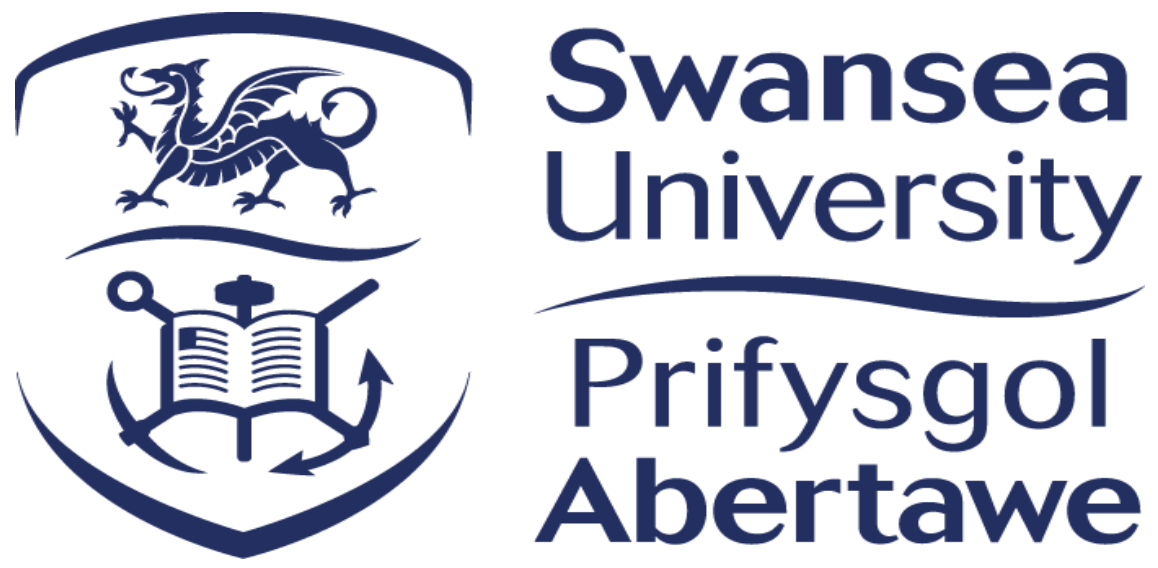

Dual practice of medical professionals in public hospitals in south-eastern Nigeria:

An economic and policy analysis

\section{Bartholomew Soludo Eze}

This thesis is submitted to Swansea University in fulfillment of the requirements for the

Degree of Doctor of Philosophy 


\begin{abstract}
Dual practice (DP) occurs when doctors employed in the public sector work concurrently in private practice. This study examines the perceived effects of DP on the public healthcare system in Enugu Urban area, Nigeria and the burden on patient finances. The study comprises a survey of 407 service users who had visited both public and private health facilities in the last year, focus groups exploring service users' perceptions of benefits and disbenefits, interviews with DP and non-DP doctors exploring why they engaged in DP or refrained from doing so, and interviews with policy makers and senior administrators on their views of DP. Additionally, sensitivity analysis was used to calculate doctors' hourly pay in the public sector and to create hypothetical estimates of the value of hours lost due to absences associated with DP. The estimated annual value of hours lost from DP to a single public tertiary care hospital was US $\$ 16,013$ (best case scenario) and US $\$ 63,399$ (worst case scenario). Benefits of DP were identified as speedier attention, reduced bureaucracy in private practice and reduction of pressure in public hospitals among others. Reported disbenefits included low commitment to public patients, late reporting and absence of doctors from work, and sharp practices. The public system was ranked higher in respect of the structure element of healthcare quality, whereas private practice performed better in the process and outcome elements of quality. The main motives for DP were seen as income enhancement followed by prestige and fringe benefits enjoyed in the public system. Reasons given by non-DPs for working exclusively in the public sector included the stress of private practice, family issues and career development. Among the different mechanisms and options proposed for managing the issue of DP in Nigeria, intramural practice appeared to command most support from policy makers and other senior stakeholders.
\end{abstract}




\section{Declaration and statements}

This work has not previously been accepted in substance for any degree and is not being concurrently submitted in candidature for any degree.

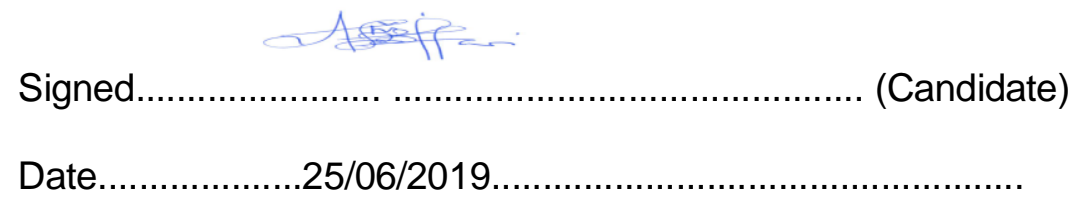

\section{STATEMENT 1}

This thesis is the result of my own investigations, except where otherwise stated.

Signed................................................. (Candidate)

\section{STATEMENT 2}

I hereby give consent for my thesis, if accepted, to be available for photocopying and for interlibrary loan, and for the title and summary to be made available to outside organisations.

Signed

(Candidate)

Date. $25 / 06 / 2019$ 


\section{Dedication}

This thesis is dedicated to my beloved mother, Roseline Eze (Nee-Orinwodoanyichifu

Iwana), who sadly passed away in the first year of this programme. 


\section{Contents}

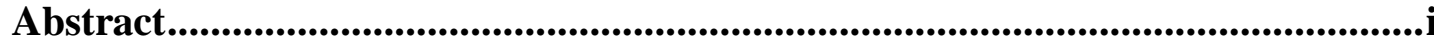

Declaration and statements ...................................................................................ii

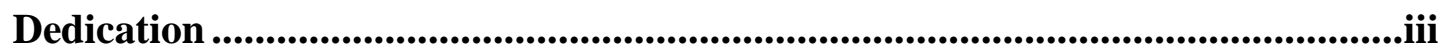

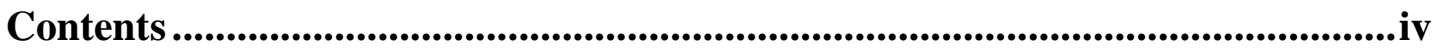

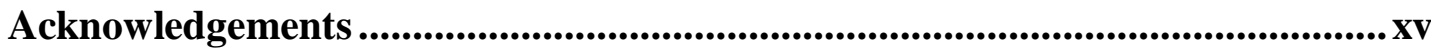

List of Tables ................................................................................................................. Xvi

List of Figures ...............................................................................................................xvii

List of Abbreviations .....................................................................................xviii

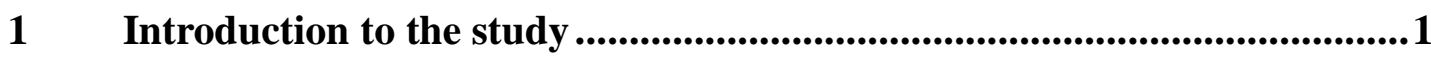

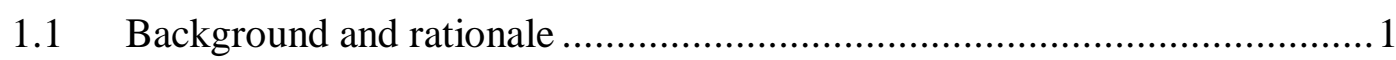

1.2 Structure of Nigerian healthcare system............................................. 7

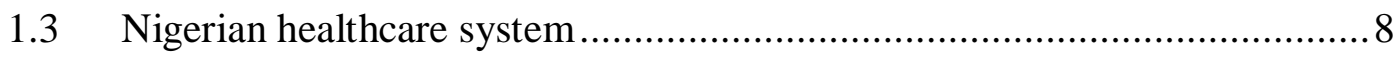

1.4 Performance of Nigerian healthcare system .................................... 10

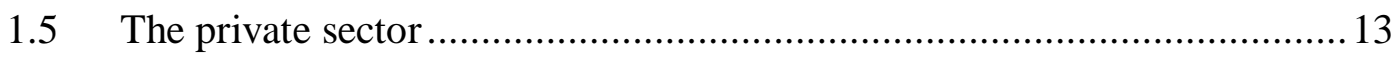

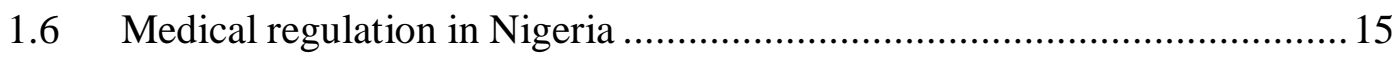

1.7 The Code of Medical Ethics in Nigeria (formerly Rules of Professional Conduct for Medical and Dental Practitioners in Nigeria) ............................................ 19

1.8 Constitutional provisions regarding private practice in Nigeria ................20

1.9 Research aims and objectives ...................................................... 22

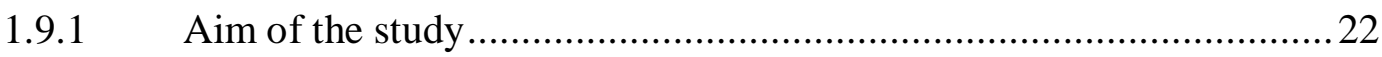

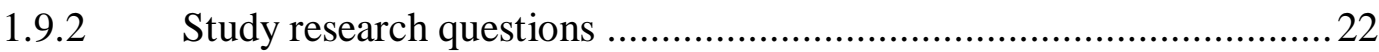

$2 \quad$ Methods and methodology ..............................................................24

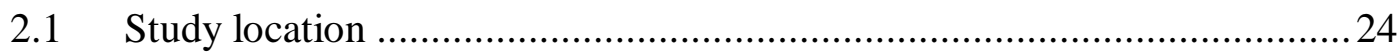

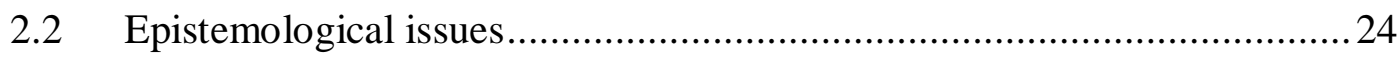

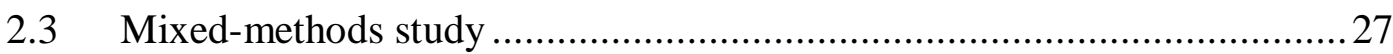

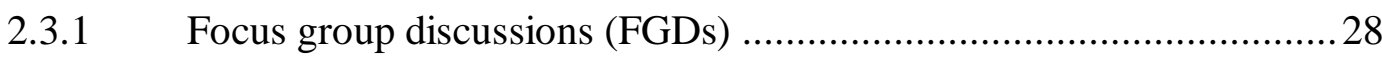

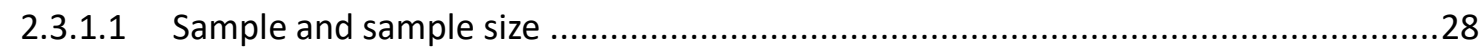

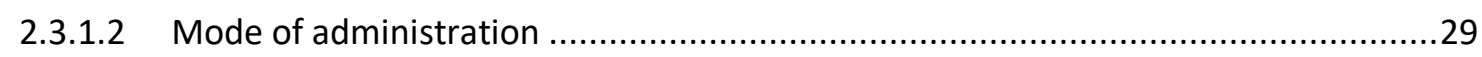

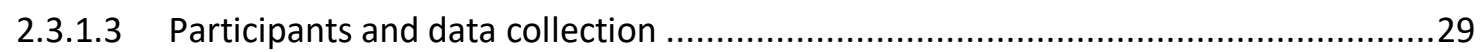

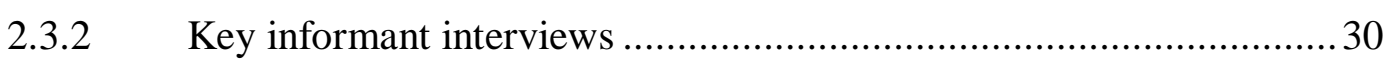

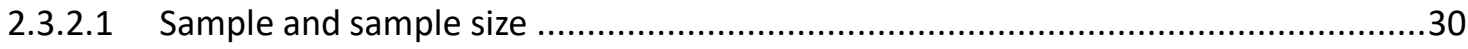




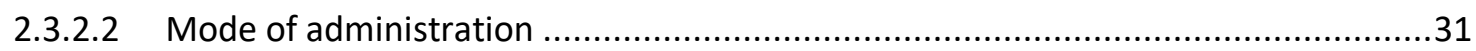

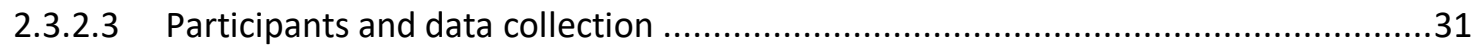

2.3.3 In-depth interviews with dual physicians and non-dual physicians ..... 32

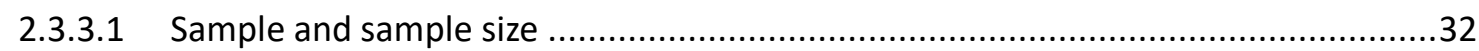

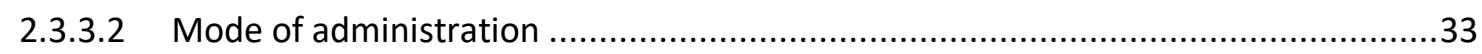

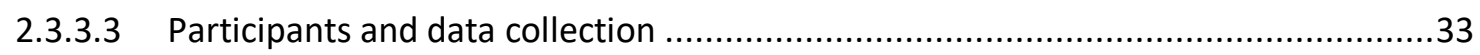

2.3.4 Ranking of motives for dual practice physicians via ranking questionnaire 33

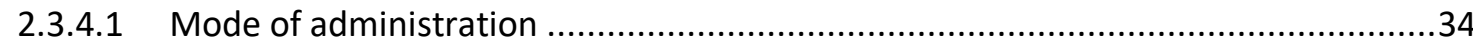

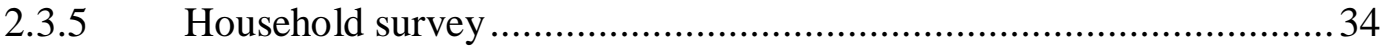

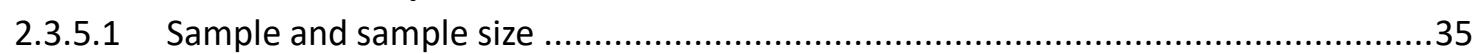

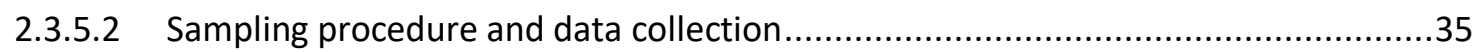

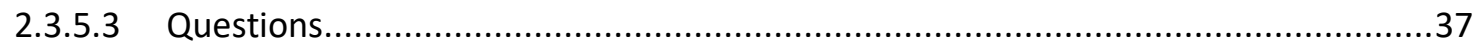

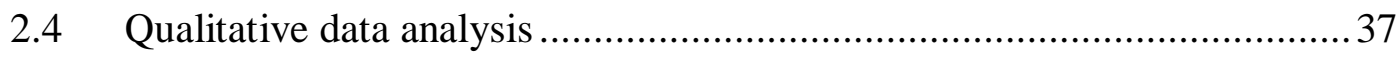

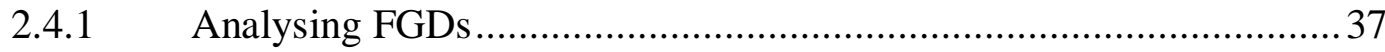

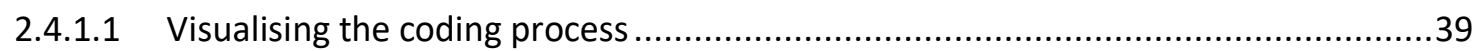

2.4.2 Key informant interviews ...................................................... 41

2.4.3 Interviews with dual physicians and non-dual physicians.................. 42

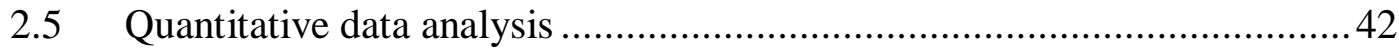

2.5.1 Ranking of motives for dual practice physicians ............................ 42

2.5.2 Household survey ................................................................. 42

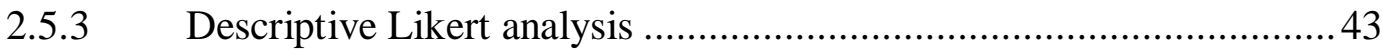

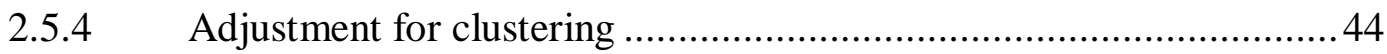

2.5.5 Sensitivity analysis of value of hours lost ......................................4

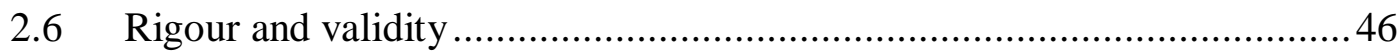

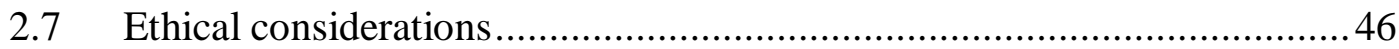

$2.8 \quad$ Literature review methodology ................................................... 47

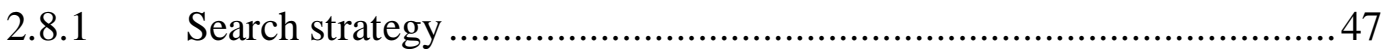

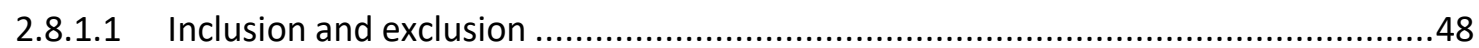

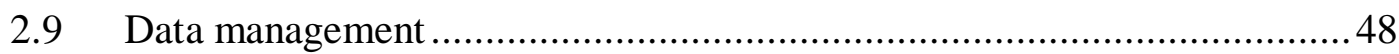

2.10 Assessing quality of study articles .....................................................48

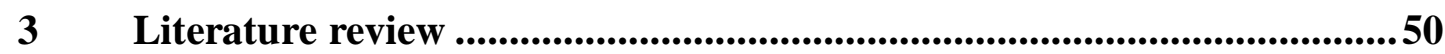

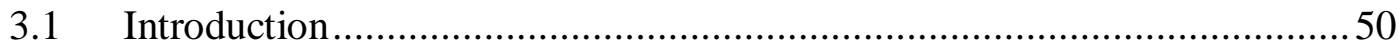

3.2 Benefits and disbenefits of dual practice among medical professionals in the health sector ..................................................................50 
3.2.1 Dual practice and access to health care .......................................50

3.2.2 Dual practice and healthcare quality ............................................52

3.2.3 Dual practice and skills development..........................................53

3.2.4 Compensation for giving up full-time private practice .......................55

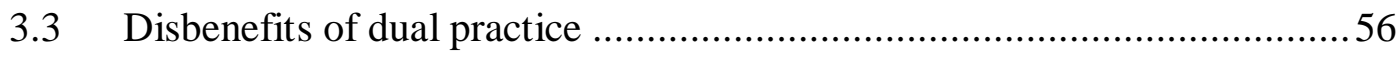

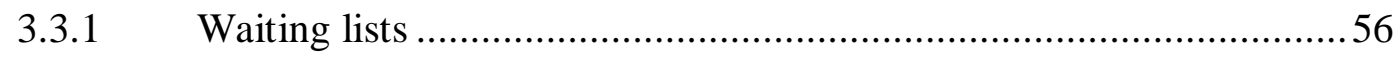

3.3.2 Competition for time/shifting of attention to private practice .............57

3.3.3 Stealing /outflow of government resources ....................................58

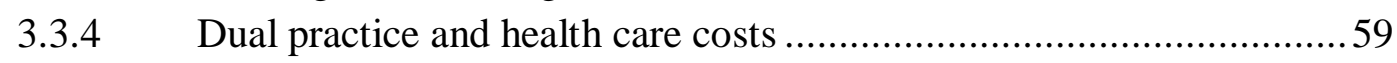

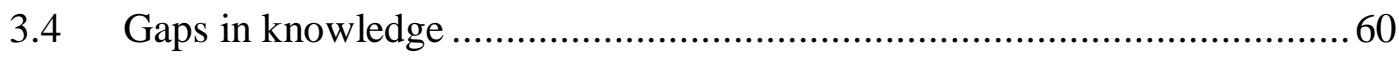

3.5 The extent of physician self-referral ..................................................... 61

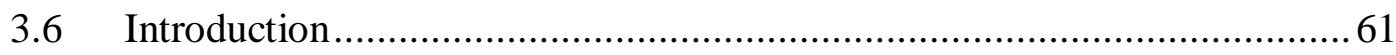

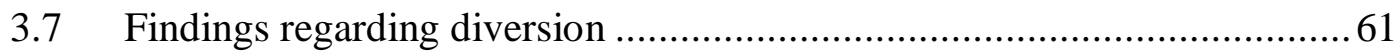

3.7.1 Dual practice and the extent of self-referral ...................................62

3.7.2 Self-referral and cream skimming...............................................6 63

3.7.3 Dual practice and indirect patient diversion ..................................6 64

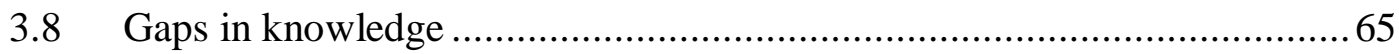

3.9 The motives for engaging in dual practice ..........................................65

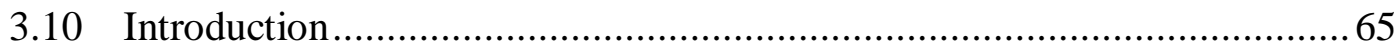

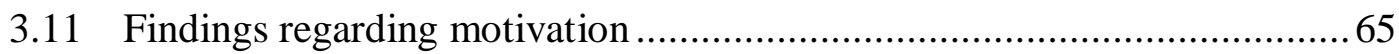

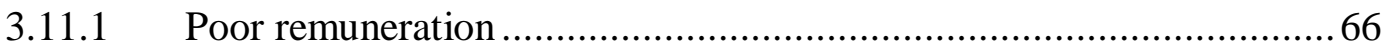

3.11.2 Confers credibility, prestige, and job security ...............................6 67

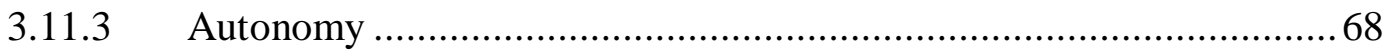

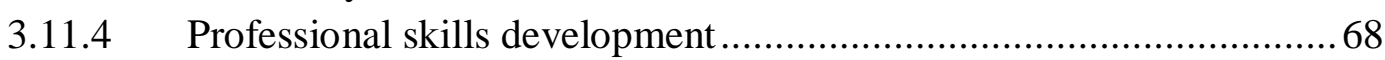

3.11.5 Growing demand for private health care .........................................69

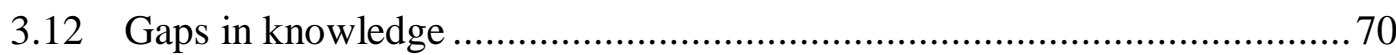

3.13 Quality of healthcare in the public sector compared with private practice .70

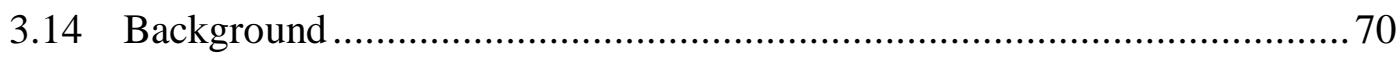

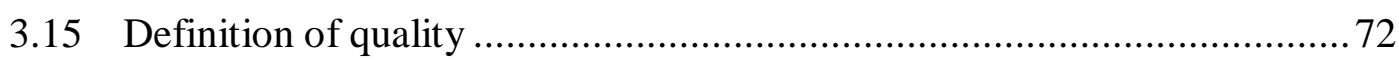

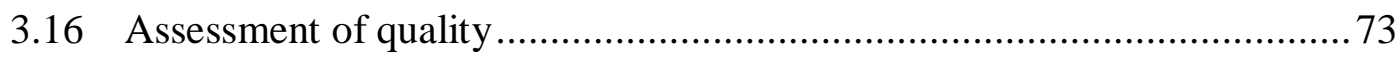

3.17 Comparing the quality of private and public healthcare......................... 77

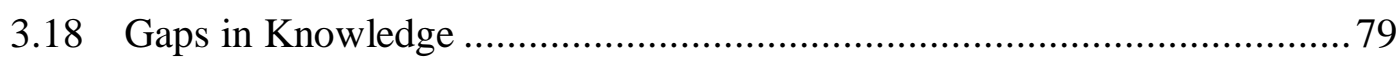

3.19 Regulatory mechanisms for dual practice in different health systems ........80 80

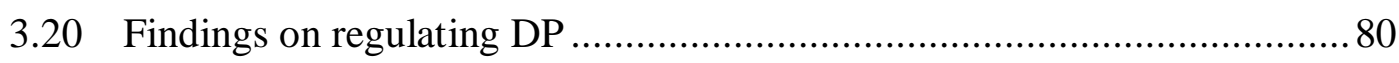

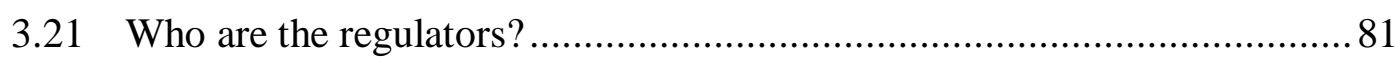




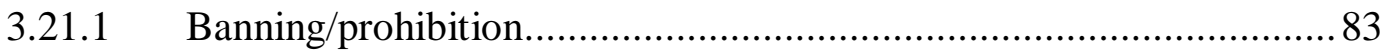

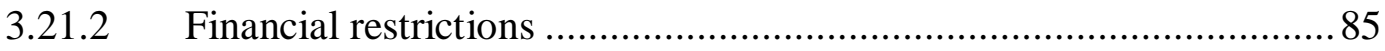

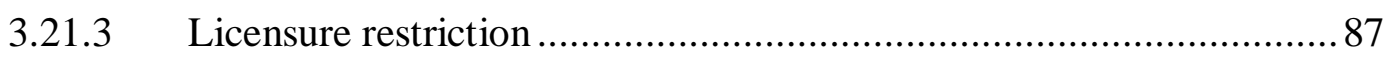

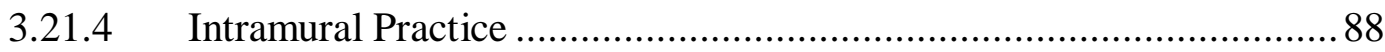

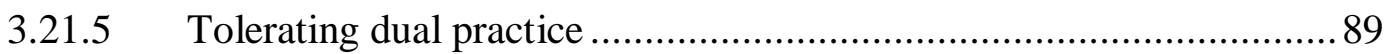

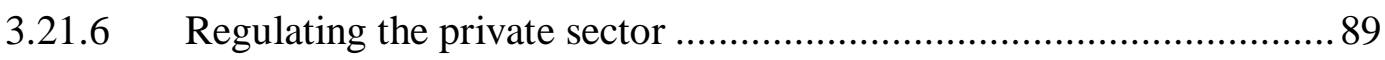

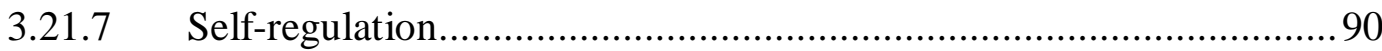

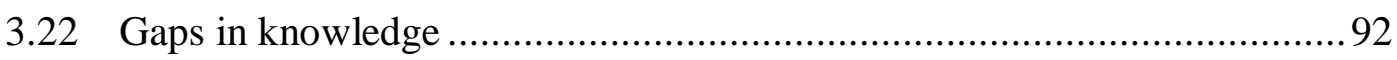

3.23 The power of the medical profession …………….................................

3.24 Findings from the profession's literature...................................................92

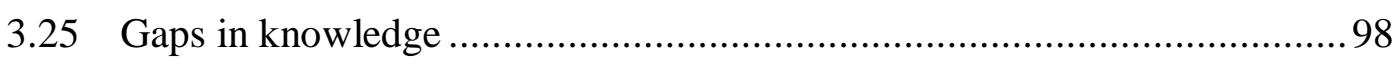

$4 \quad$ Does dual practice bring benefits? ..................................................................99

4.1 Benefits of dual practice in the health sector ..............................................99

4.2 Physicians' and stakeholders' socio-demographic Characteristics............ 100

4.3 Socio-demographic characteristics of non-dual practice physicians 101

4.4 Socio-demographic characteristics of policymakers/stakeholders............. 102

4.5 Socio-demographic characteristics of FGD participants ............................ 102

4.5.1 Socio-demographic characteristics of FGD female group1 participants 102

4.5.2 Socio-demographic characteristics of FGD female group 2 participants 103

4.5.3 Socio-demographic characteristics of FGD male group 3 participants 104

4.5.4 Socio-demographic characteristic of FGD male group 4 participant.....104

4.6 Descriptive Likert analysis of benefits of DP..................................

4.7 Perspectives of respondents on benefits of dual practice in the health sector 109

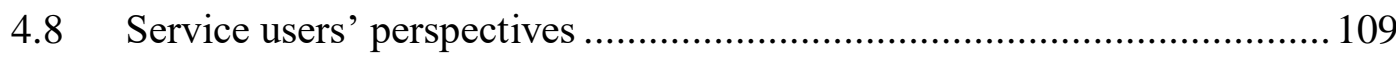

4.8.1 Easier payment plans for private patients ............................................... 109

4.8.2 An enhanced doctor/patient relationship .......................................... 111

4.8.3 Availability of dual practitioners in private practice........................... 112

4.9 Doctors' perspectives on benefits of DP .............................................. 112

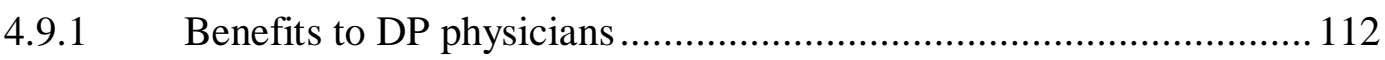

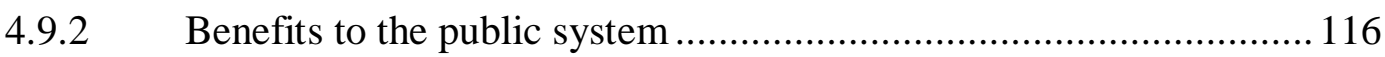

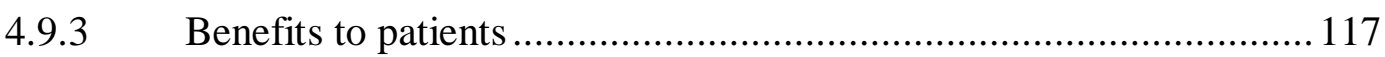

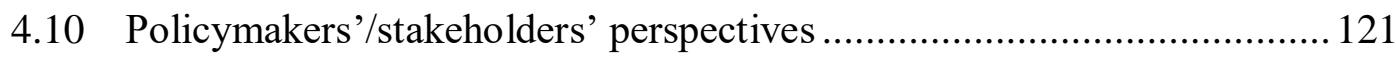

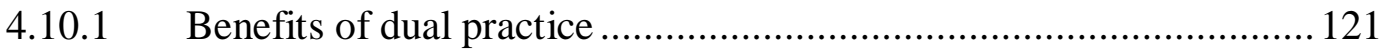

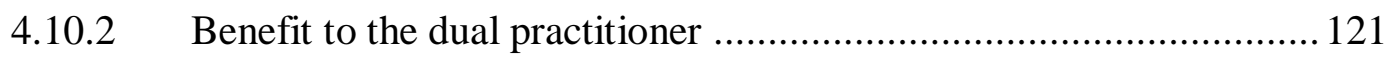

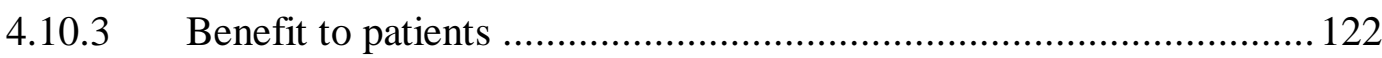

4.10.4 DP is beneficial to the private sector................................................ 123 
4.10.5 Policymakers' perceptions of dual practice benefit ....................... 124

4.11 Chapter summary/conclusion......................................................... 124

5 Disbenefits of dual practice .......................................................................126

5.1 Disbenefits of dual practice in the health sector .................................. 126

5.2 Disbenefits of dual practice to service users ........................................ 126

5.2.1 Descriptive Likert analysis of disbenefits of DP to service users ..... 126

5.3 Impacts of dual practice in terms of the burden on patients' finances ...... 128

5.3.1 Cost of treatment in private hospitals when referred from the public system 128

5.3.2 Estimated mean cost of treatment in public hospitals for the same health conditions for which patients were referred to the private sector.....

5.4 How did referred patient group cope with paying for private treatment? .132

5.5 Impacts of dual practice in terms of value of hours lost to the public system 133

5.6 Hypothetical estimates of on call hours lost to dual practice and financial implications in a tertiary hospital

5.7 Disbenefits of dual practice through the eyes of respondents ................. 136

5.7.1 Service users' perspectives on disbenefits..................................... 137

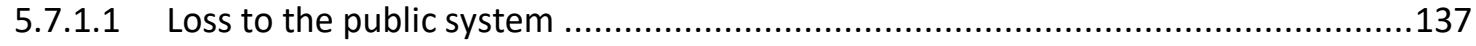

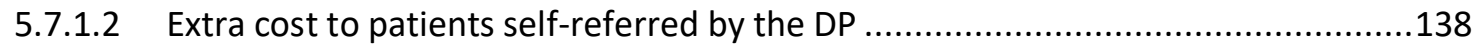

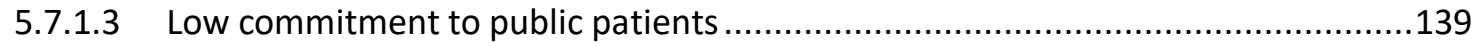

5.7.1.4 Low commitment to public hospital work........................................................140

5.8 Doctors' perspectives on disbenefits of DP .......................................... 141

5.8.1 Disbenefits to patients ............................................................... 141

5.8.2 Dual practice affects medical training in the public system .............. 141

5.8.3 Low productivity in the public sector............................................ 143

5.8.4 Sharp practices in the private sector............................................. 145

5.9 Policymakers'/stakeholders' perspectives on disbenefits of DP .............. 147

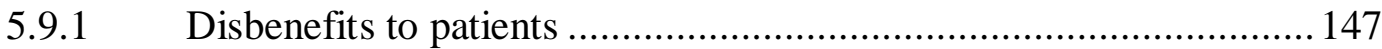

5.9.2 High cost of care in the private sector .......................................... 147

5.9.3 Denying services to public patient ............................................... 148

5.9.4 Sharp practice in the private sector .............................................. 148

5.9.5 Suboptimal quality of service ................................................... 149

5.9.6 Disbenefits to the public system ..................................................... 149

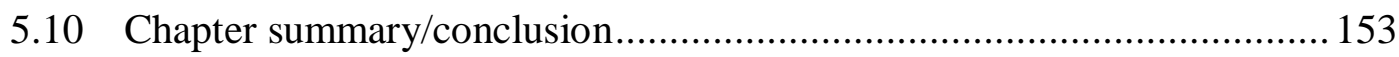

6 Referral patterns by public sector providers ........................................ 155

6.1 Extent of physician self-referral ......................................................... 155 


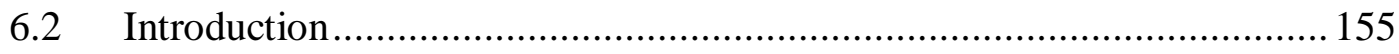

6.3 Respondents' socio-economic characteristics........................................... 155

6.3.1 Socio-demographic characteristics of respondents ........................... 155

6.3.2 Socio-economic status of patients referred ....................................... 158

6.3.3 Age groups of patients referred from public system to private sector 158

6.3.4 Proportion of male and female patients referred from public to private hospitals .159

6.3.5 Referral patterns used by public sector providers ............................. 160

6.4 Perspectives on self-referral................................................................. 161

6.5 Service users' perspectives on self-referral ............................................. 162

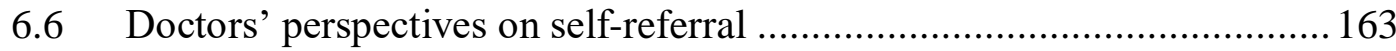

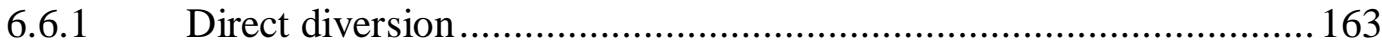

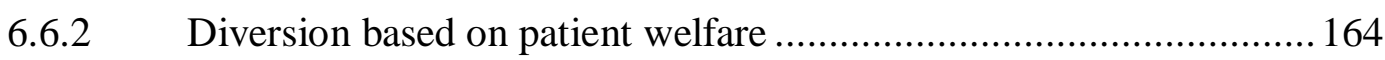

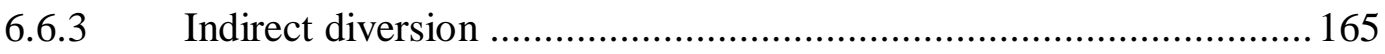

6.7 Policy makers'/stakeholders' perspectives on the diverting of patients to private care 166

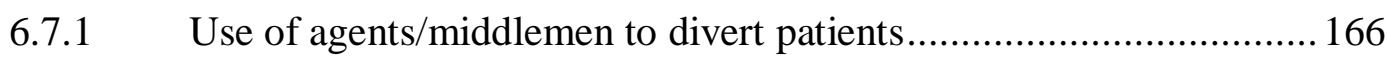

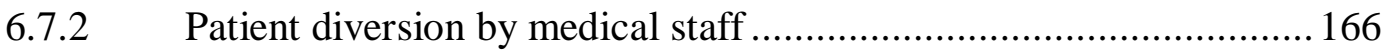

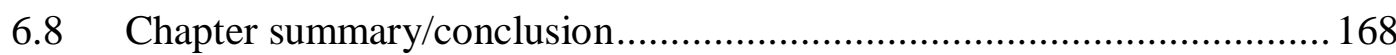

7 Are there differences in quality of healthcare in public system and private practice managed by dual practice physicians? ...................................................169

7.1 Comparing differences in quality of healthcare in public system and private practice managed by dual practitioners. 169

7.2 Descriptive Likert analysis of service users' experience of healthcare quality in public system and private practice managed by dual practice physicians ............ 169

7.3 How do respondents perceive healthcare quality? ..................................... 175

7.4 Respondents' perceptions of healthcare quality........................................ 175

7.5 Comparing quality of healthcare in public system and private practice using

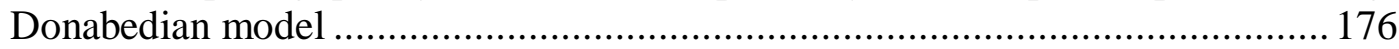

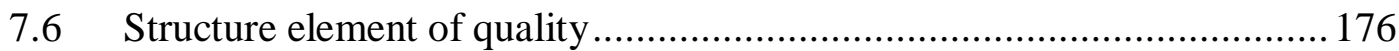

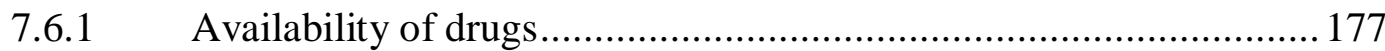

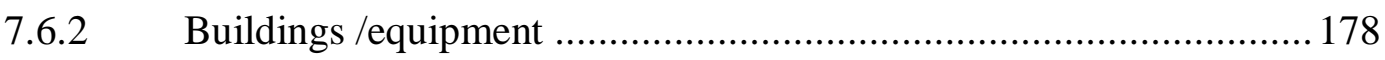

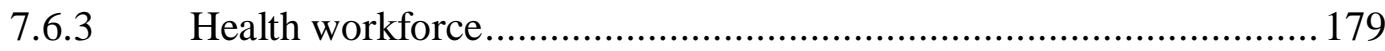

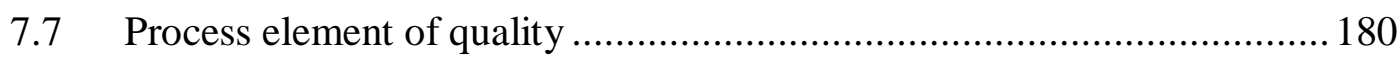

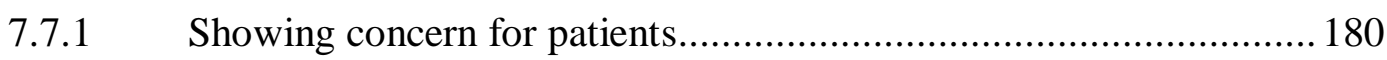

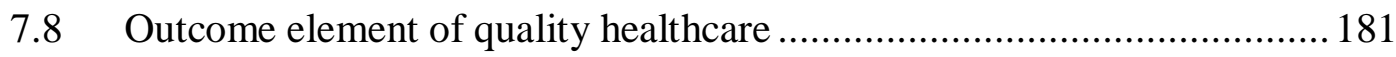


7.8.1 Positive outcome element experience ........................................ 182

7.8.2 Negative outcome element experience ....................................... 183

7.8.3 Treatment satisfaction in public and private sector......................... 183

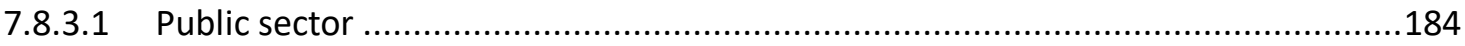

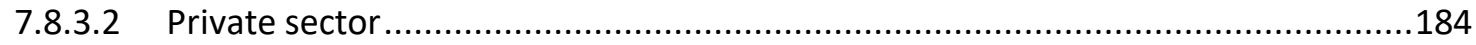

7.9 Chapter summary/conclusion..................................................... 185

8 Motives for dual practice in the public system .......................................... 186

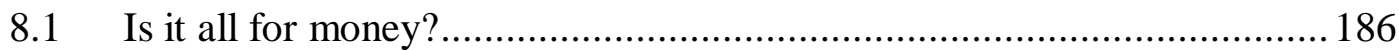

8.1.1 Ranking the motives for DP by government-employed doctors engaged in dual

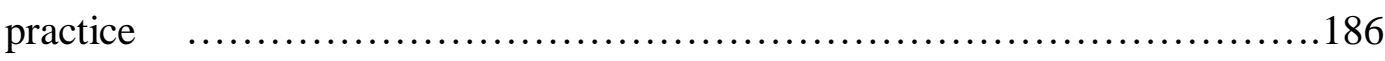

8.1.2 Descriptive Likert analysis of service users' perceptions of DP .......187

8.1.3 Cluster analysis of study locations ................................................ 189

8.2 Perspectives on motives for dual practice .......................................... 191

8.3 Service users' views on doctors engaged in dual practice...................... 191

8.3.1 DPs make financial gain from private practice ............................... 191

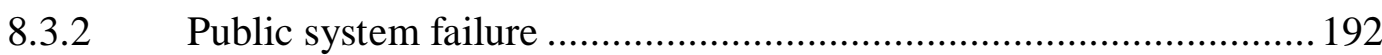

8.4 Doctors' views on dual practice by public sector doctors ...................... 192

8.4.1 Escaping bureaucracy in the public system ................................... 193

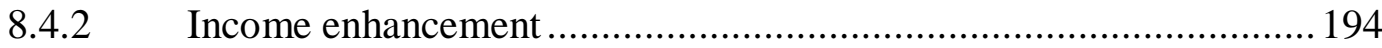

8.4.3 Poor working conditions in the public sector ................................ 195

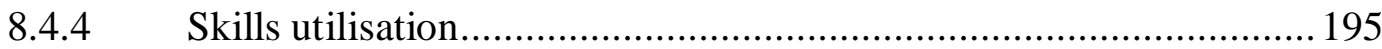

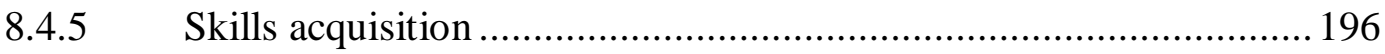

8.5 Policy makers'/stakeholders' views on doctors engaged in dual practice. 197

8.5.1 Bureaucracy in government hospitals ........................................ 197

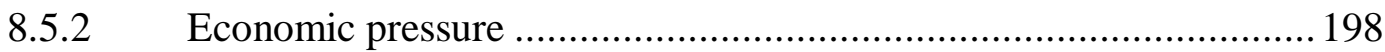

8.5.3 Poor conditions in public hospital............................................. 199

8.5.4 Community demand for private clinics .......................................201

8.6 What conditions could make a doctor work exclusively in the public system? 202

8.6.1 Reducing bureaucracy in public hospitals .................................... 202

8.6.2 Opportunities for conferences and medical updates ......................203

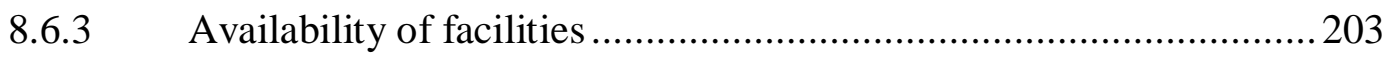

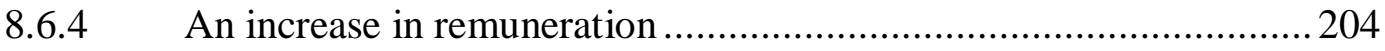

8.6.5 Improved conditions of work in the public sector ........................ 205

8.7 Reasons for an exclusive public work by non-dual practice physicians ...206

8.7.1 Avoiding the stress in private practice ...........................................206

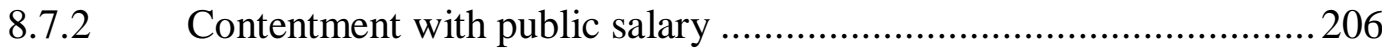

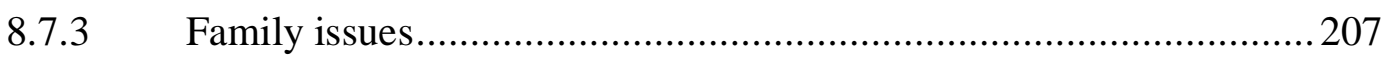


8.7.4 Career development.................................................................. 208

8.8 Policy of dual practice of medical professionals in Nigeria .....................209

8.9 Challenges in combating dual practice in Nigeria ...............................209

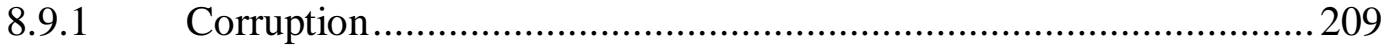

8.9.2 Funding and logistical challenges ............................................ 210

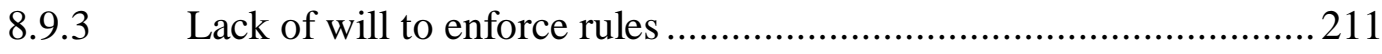

8.9.4 Poor remuneration of doctors..................................................... 212

8.9.5 Weak sanctions for rule-breakers.............................................. 212

8.10 Perspectives on mechanisms/options to manage DP in Nigeria and challenges for reform. 213

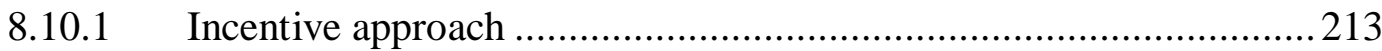

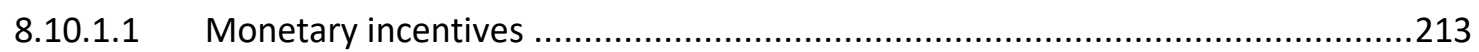

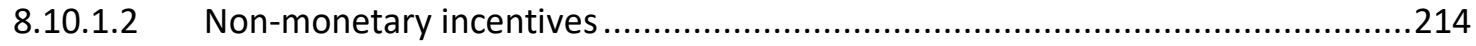

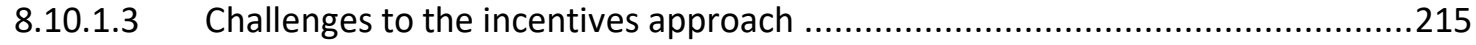

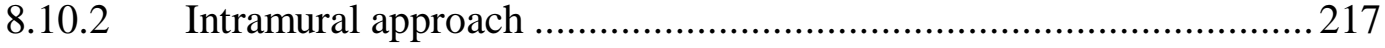

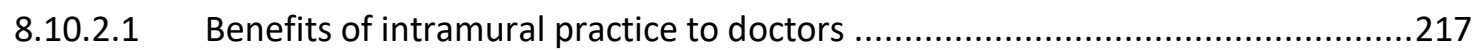

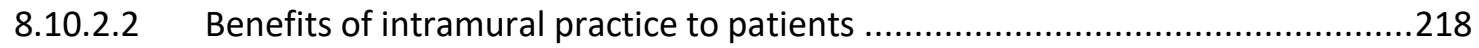

8.10.2.3 Benefits of intramural practice to public healthcare facilities..........................219

8.10.3 Challenges of intramural practice ..............................................221

8.10.3.1 Doctors' implicit opposition to the intramural approach.................................222

8.10.4 Allowing dual practice as the norm..............................................2223

8.10.5 Limiting types of services offered in the private sector ...................224

8.10.5.1 Negative effects of limiting types of services in the private sector ...................224

8.10.6 Challenges in limiting types of services offered in the private sector 225

8.10.7 Banning dual practice .............................................................. 226

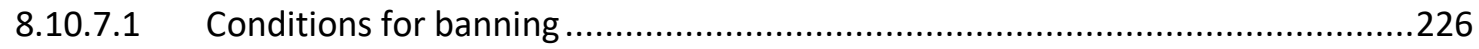

8.10.8 Consequences of banning dual practice in Nigeria ......................... 227

8.10.9 Policy makers' views on banning dual practice.............................. 229

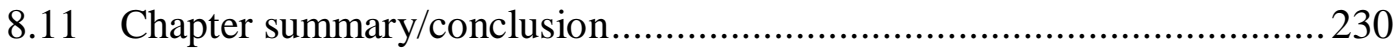

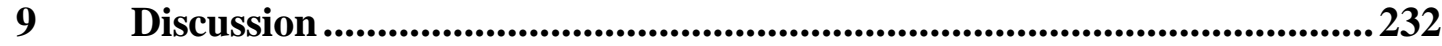

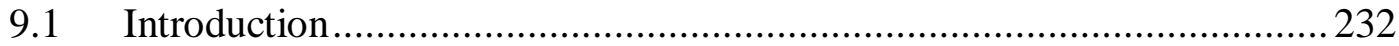

9.1.1 Revisiting the research questions in this study ............................2232

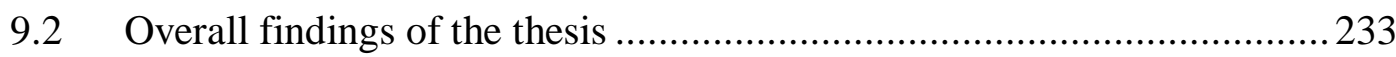

9.3 Research question 1a: What are the benefits of DP in the health sector in Nigeria?

9.3.1 Speedier attention to private patients ......................................... 233 
9.3.2 Availability of DP physicians in private practice ......................... 234

9.3.3 Enhanced doctor/patient relationship in private practice ................2.234

9.3.4 DP helps patients to avoid bureaucracy in the public sector .............235

9.3.5 Reduction of pressure in public hospitals .................................. 235

9.3.6 Paying in instalments for private practice ...................................2235

9.3.7 Extra income for dual practitioners ...............................................2236

9.3.8 Professional development of DP doctors...................................... 236

9.4 Research question $1 \mathrm{~b}$ : What are the disbenefits of dual practice in the health sector in Nigeria? 237

9.4.1 Low commitment to public patients..............................................2237

9.4.2 Late reporting and absence of doctors from work ......................... 237

9.4.3 DP affects medical training in government hospitals...................... 237

9.4.4 Sharp practice in the private sector ............................................. 238

9.4.5 Impacts of dual practice in terms of the burden on patients' finances 238

9.4.6 Expenditures for treatment in the private hospitals when referred from the

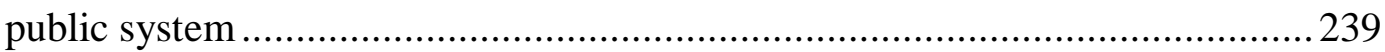

9.4.7 Estimated mean cost of treatment in public hospitals for the same health conditions for which patients were referred to the private sector....................239

9.4.8 The extent of self-rated financial impact of private treatment for the referral

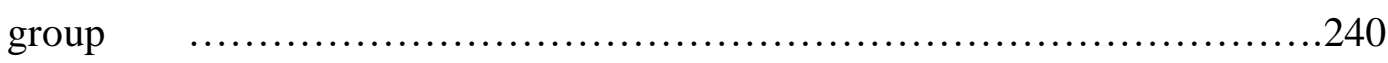

9.4.9 Coping strategies for paying for private treatment by the referral group240

9.4.10 Impact of dual practice in terms of value of hours lost to the public system due to absences by dual practitioners from normal routine ................................... 241

9.4.11 Best-case and worst-case scenarios ............................................. 242

9.4.12 Impact of dual practice in terms of value of hours lost to the public system due to absences by dual practitioners from on call hours ...................................... 243

9.4.13 Best-case and worst-case scenarios..............................................2 243

9.5 Research question 2: What is the extent of physician self-referral in public hospitals

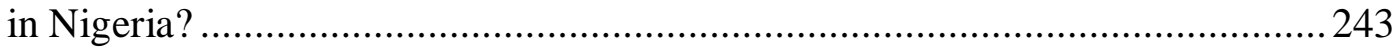

9.5.1 Age group of patients referred from public hospitals......................244

9.5.2 Proportion of male and female referred from public hospitals .......... 244

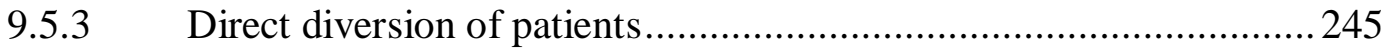

9.5.4 Indirect diversion of patients ...................................................... 245

Research question 3: How does the perceived quality of healthcare in the public sector compare with quality of healthcare in private healthcare facilities managed by dual

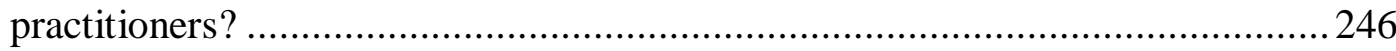

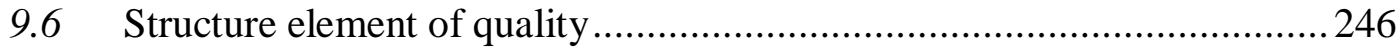

9.6.1 Better infrastructure/ equipment and availability of drugs in public hospitals than in private practice 246 
9.6.2 Extended business hours, but fewer qualified healthcare staff in private practice

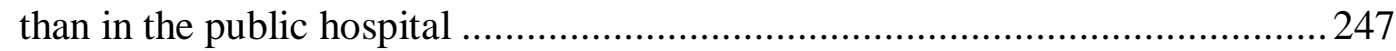

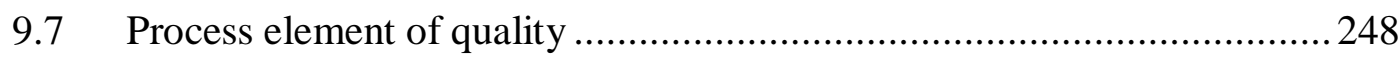

9.7.1 Better rapport with doctors in private practice ...............................248

9.7.2 Less waiting time in private practice/ more time to explain things in a way

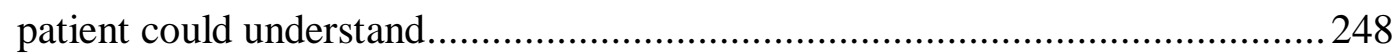

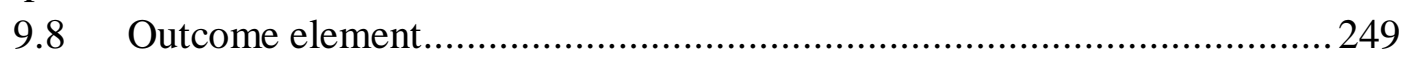

9.8.1 Better health outcomes in private practice...................................... 249

9.9 Research question 4: What are the motives that lead Nigerian doctors to engage in dual practice, or refrain from doing so? ..........................................................2 250

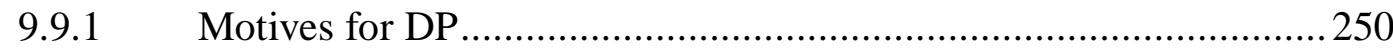

9.10 Perception of service users of doctors engaged in DP ......................... 251

9.11 Doctors' views of private practice .................................................... 251

9.11.1 What conditions could make a doctor work exclusively in the public system? .252

9.12 Reasons for an exclusive public work by non-dual practice physicians ...252

9.13 Research question 5: What are stakeholders' views on the implementation of existing regulations on DP, alternative regulatory mechanisms /options for controlling dual practice and the challenges for reform? 254

9.13.1 Stakeholders' views on challenges in combating dual practice in Nigeria 254

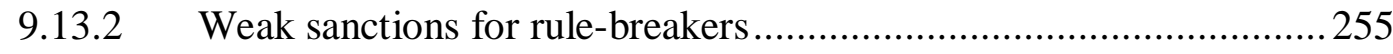

9.13.3 Poor remuneration of doctors.................................................... 255

9.14 Perspectives on mechanisms/options to manage DP in Nigeria and challenges for

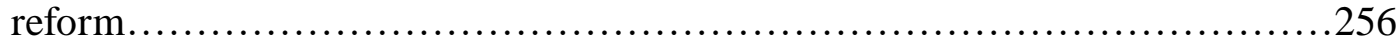

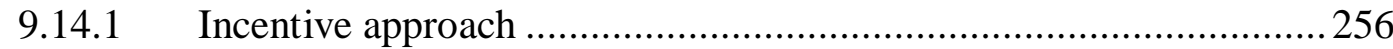

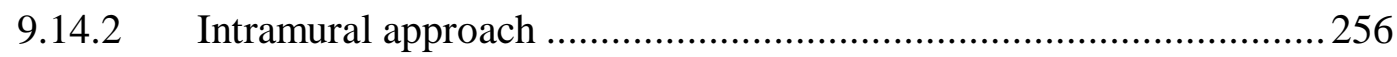

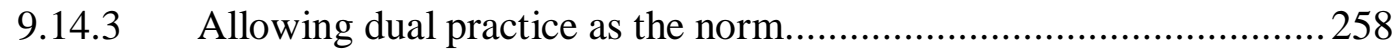

9.14.4 Limiting types of services offered in the private sector ..................258

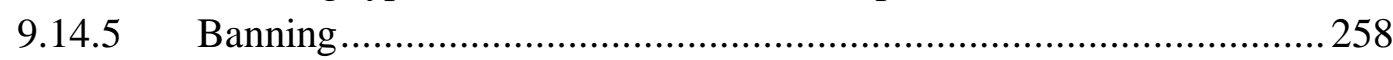

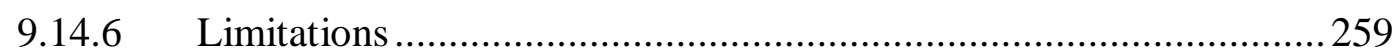

9.15 Main contributions of the thesis to knowledge ....................................263

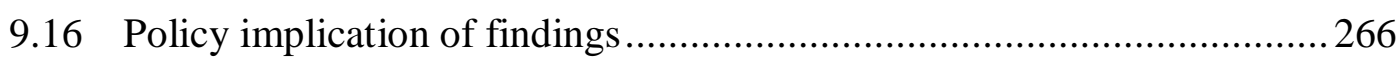

10 Conclusions and recommendations ................................................2270

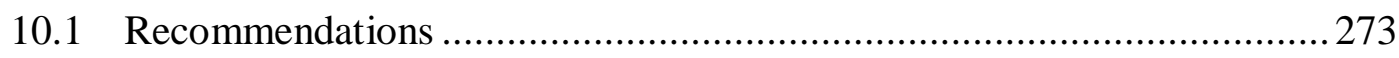

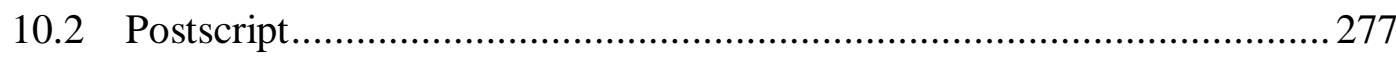

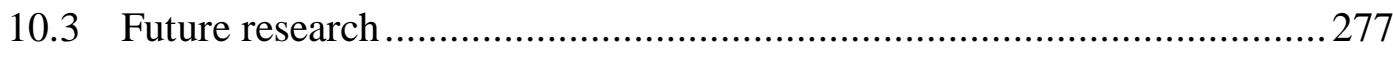

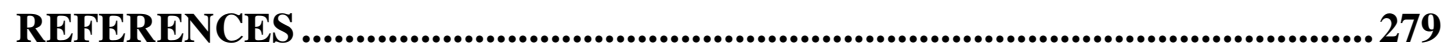




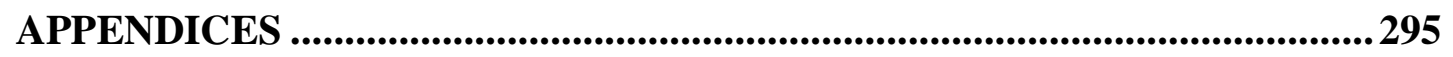

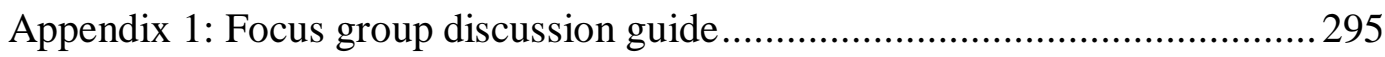

Appendix 2: Participant information sheet.................................................297

Appendix 3: Interview guide for policy makers/stakeholders..........................2 298

Appendix 4a: In-depth interview guide for dual practitioners (PART 1) ...........298

Appendix 4b: Ranking on motives for DP by dual practice doctors PART 2..299

Appendix 5: In-depth interviews guide for non-dual practitioners ....................300

Appendix 6: Questionnaire on dual practice of medical professionals in public health

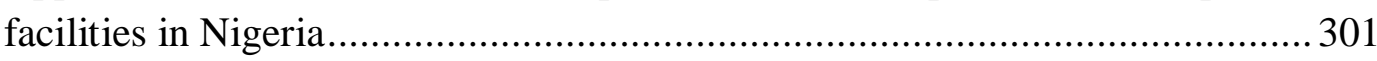

Appendix 7: Visual representation of a theme on perception of quality healthcare (Focus group discussions with service users) ......................................................... 316

Appendix 8: Visual representation of a theme on intramural practice (policy makers' interview) ....................................................... 317

Appendix 9: Visual representation of a theme on motives for dual practice (doctors'

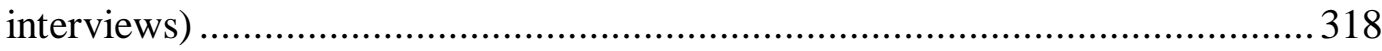

Appendix 10: Consolidated Medical Salary Structure (doctors' salary with other

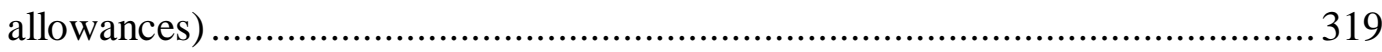

Appendix 11: Computation of different hypothetical scenarios using Excel Worksheet

(Example) ................................................... 320

Appendix 12: Five hours weekly loss to absences by DPs per year ................. 320

Appendix 13: Eight hours weekly loss to absences by DPs per year ............... 322

Appendix 14: 10 hours weekly loss to absences by DPs per year......................322

Appendix 15: 12 hour's weekly loss to absences by DPs per year ...................323

Appendix 16: 15 hours weekly loss to absences by DPs per year......................323

Appendix 17: 20 hour's weekly loss to absences by DPs per year ....................324

Appendix 18: Consolidated Medical Salary Structure (on-call hour allowance for doctors)

325

Appendix 19: Five hours weekly loss to absences by DPs on on-call hour per year 326

Appendix 20: Six hours weekly loss to absences by DPs on on-call hour per year.....327

Appendix 21: Seven hours weekly loss to absences by DPs on on-call hour per year 327

Appendix 22: Eight hours weekly loss to absences by DPs on on-call hour per year 328

Appendix 23: Nine hours weekly loss to absences by DPs on on-call hour per year....329

Appendix 24: 10 hours weekly loss to absences by DPs on on-call hours per year......330

Appendix 25: Ethics from UNTH, Ituzu-Ozalla ............................................ 331

Appendix 26: Ethics from the Enugu State Ministry of Health ......................... 332

Appendix 27: Ethics from the College of Human and Health Sciences ............. 333

Appendix 28: Participant consent form....................................................... 334

Appendix 29: An overview of some of the main studies reviewed ................... 335 


\section{Acknowledgements}

The success of this programme drew supports from different individuals and organizations. First and foremost, I wish to sincerely thank my first supervisor, Professor David Hughes for his tireless efforts and commitments to the supervision of this work. I also, wish to thank Professor Ceri Phillips, my second supervisor, for his useful comments in this work. Honestly, I appreciate your inputs.

I would not forget the overwhelming support of my dear wife, Chinenye, throughout this whole academic journey. On several occasions, I have had to discomfort you and my little boy, Kobby, who sometimes would wake up in the night only to find out that his daddy was not there to cuddle him. I do not forget all the sacrifices you have made to see this project achieve a successful conclusion. I would ever remember my little angel, Chioma (Mama), who was busy kicking in the womb and praying for her daddy's success during the writing-up stage of this work.

I wish to express my gratitude to the Medical and Dental Council of Nigeria for granting me an interview and providing the information that has helped to shape this thesis. To the PGR Academy Manager, Maria Davies, I thank you for your assistance throughout my studies in the College of Human and Health Sciences. I must give credit to Dr. Mari Jones whose statistical knowledge benefited this work tremendously.

To Thornton Frances, I thank you immensely for the one-on-one meetings concerning the formatting of the final draft of this thesis, and to my fellow $\mathrm{PhD}$ candidates in the postgraduate office in Vivian Tower, I thank you for your various efforts to support me in one way or another.

I am also grateful to Professor Obinna Onwujekwe and Dr. Mazi Ogbonnia Ochonma of the Department of Health Administration and Management, University of Nigeria, Enugu Campus for their assistance.

Finally, for other numerous individuals, who directly or indirectly contributed to the realization of this dream, space may not be enough to convey my heartfelt appreciation one after the other, however, please accept my sincere thanks. 


\section{List of Tables}

Table 1: Socio-demographic characteristics of dual practitioners ...................................... 100

Table 2: Socio-demographic characteristics of non-dual practitioners ................................. 101

Table 3: Socio-demographic characteristics of policymakers/stakeholders ......................... 102

Table 4: Socio-demographic characteristics of participants (1) ....................................... 103

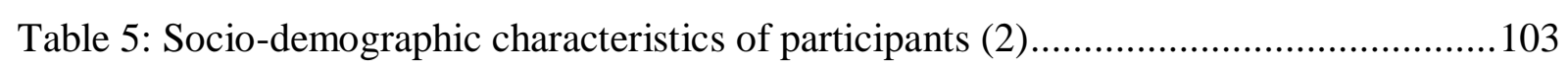

Table 6: Socio-demographic characteristics of participants (3)........................................ 104

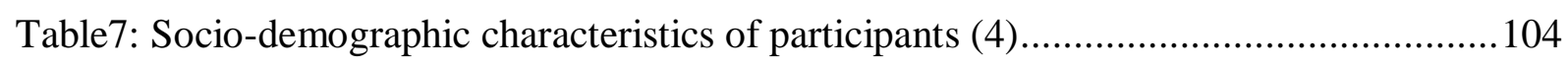

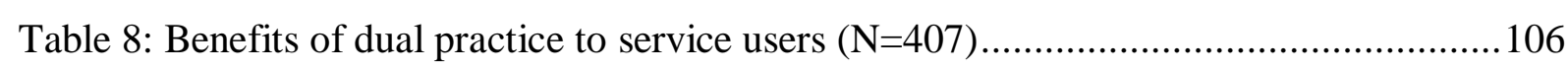

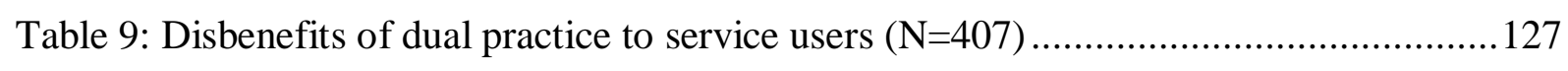

Table 10: Respondents' expenditures for treatment in private hospitals as a result of referral

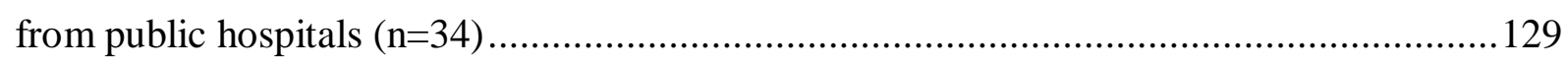

Table 11: Estimated mean cost of treatment in public hospitals for the same health conditions

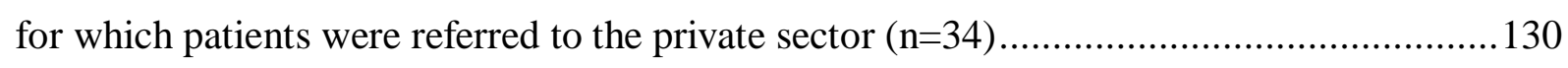

Table 12: Self-rated financial impact of private treatment for patient group referred from

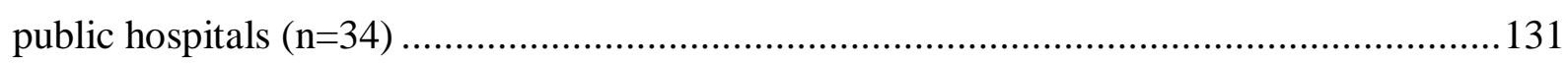

Table 13: Coping strategies for paying for private treatment by the referral group $(n=34) \ldots 132$

Table 14: Number of referrals and non-referrals and their Socio-demographic characteristics

Table 15: Comparing NDHS and HH survey data....

Table 16: Number and percentage of respondents in different SES groups referred from the

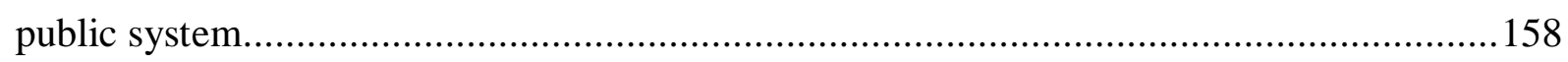

Table 17: Comparison of structure element of healthcare quality between DPs' private practice and public practice $(\mathrm{N}=407)$

Table 18: Comparison of process element of healthcare quality between DP's private practice and public practice $(\mathrm{N}=407)$

Table 19: Comparison of outcome element of healthcare quality between DPs private practice

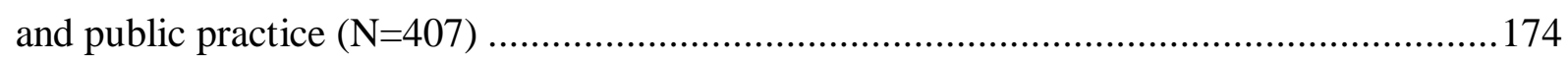

Table 20: Mean scores for self-reported motives for dual practice by dual practitioners $(n=15)$ 
Table 21: Service users' perceptions of government doctors who engage in dual practice and

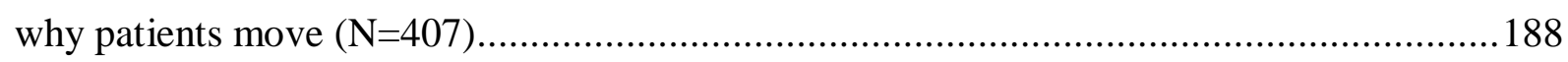

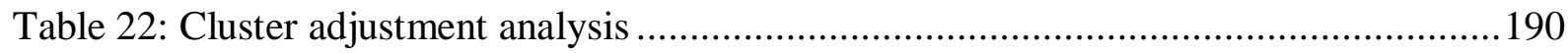

\section{List of Figures}

Figure 1: Summary of 'stages' of data analysis for the FGDs and IDIs ...............................39

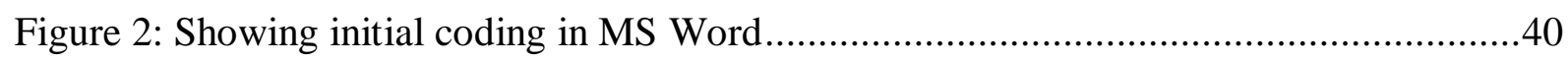

Figure 3: Showing initial codes imported into Excel ................................................. 41

Figure 4: Compares mean cost of treatment for the same health conditions of patients referred

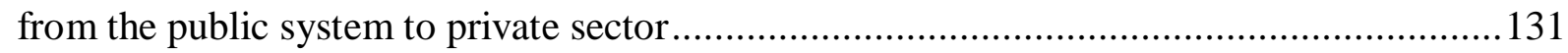

Figure 5: Combined hypothetical estimates of total value of hours lost for weekly absences for

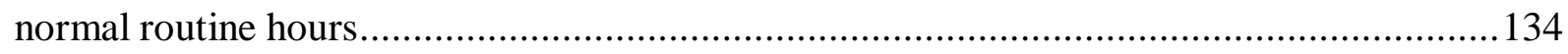

Figure 6: Combined hypothetical estimates of value of hours lost for weekly absences for on

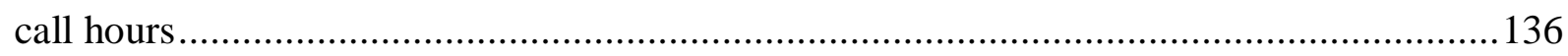

Figure 7: Age group of patients referred from the public system .................................. 158

Figure 8: Proportion of male and female patients referred from the public system...............159

Figure 9: Strategies used by healthcare providers to refer patients from the public system ..161 


\section{List of Abbreviations}

CMAC Chairman of the Medical Advisory Committee

CMD Chief Medical Director

DCMAC Deputy Chairman of the Medical Advisory Committee

DP Dual practice

DPs Dual practitioners

DSS Directorate of State Services

FGD Focus group discussion

FMOH Federal Ministry of Health

FWACP Fellowship of the West African College of Physicians

HH Household

IDIs In-depth interviews

JOHESU Joint Health Sector Unions

LMICs Low and Middle-Income Countries

MDCN Medical and Dental Council of Nigeria

NAFDAC National Agency for Food and Drug Administration and Control

NHIS National Health Insurance Scheme

NHS National Health Service

NMA Nigeria Medical Association

NPC National Population Commission

OOP Out-of-Pocket Payment

OPD Outpatient Department

PCA Principal Component Analysis

PHCs Primary Healthcare Centres

SES Socio-economic status group

SMOH State Ministry of Health

SOPs Standard Operating System

UK United Kingdom

USD United States Dollar (\$) 


\section{Introduction to the study}

\subsection{Background and rationale}

Dual practice (DP) occurs when medical professionals, who have contracts of employment with the public sector, work concurrently in the private sector (Abera, Alemayehu, \& Henry, 2017; Eggleston \& Bir, 2006; García-Prado \& González, 2011; Hipgrave \& Hort, 2013; Jumpa, Jan, \& Mills, 2007). Some authors (Baah-Boateng, Adjei, \& Abena, 2013; James, Richard, \& Mauricio, 2000; Karen \& Jean, 1998; Steven \& Bazzoli, 1985) use a different name - moonlighting - to define engagement in public and private sector work simultaneously. Others, (Berman \& Cuizon, 2004; Dickey, et al., 2011; Jan et al., 2005) use dual job holding and multiple jobs holding respectively to explain concurrent engagement in public and private sector work. These different terms, therefore, convey much the same meaning. Dual practice occurs among different categories of workers (Hipple, 2010), and it is evident in both industrialised (Helen et al., 2014) and industrializing (Macq, Ferrinho, Brouwere, \& Lerberghe, 2001) countries.

The engagement of medical professionals in both public and private sector work is increasingly gaining the attention of many governments in developed and developing countries alike (Kiwanuka, et al., 2011). Some authors have suggested that it is in the interest of government to acknowledge the existence of DP by investigating its potential impact on public service provision (Berman \& Cuizon, 2004; Jumpa et al. 2007). Ignoring its existence would not prevent any impact on the health system from occurring.

The important element often neglected in discussing private vs public medical care is distinguishing private sector health care, where medical doctors and nurses operate as independent private medical entrepreneurs, from the engagement of public sector workers in private practice as income top-up activity (Ferrinho et al., 1998). The crux of the argument about DP comes down to whether it has positive or negative consequences. It has been argued that the consequences associated with DP may differ across different health systems depending on the strength of their regulatory mechanisms, nature of the medical labour market, and physicians' motivation (Socha \& Bech, 2012). For example, there has been much public debate in Denmark about whether dual practice has stimulated the growth of the private sector, and consequently, the move for the amendment of the Health Act (Socha \& 
Bech, 2012). In that country this growth is seen as a threat to the running of the public healthcare system.

Nevertheless, the contribution of the private sector cannot be overstated in low-and -middle income countries, and arguably in high income countries as well (Hanson, et al., 2008). It has been shown that in the developing world, the private sector provides services to a wide range of socio-economic groups, including in the view of some authors the poor (Loh, Ugarte-Gilb, \& Darko, 2013). While the poor may gain access to the private sector, researchers need to consider the financial impact of paying out of pocket, which may mean that this is limited in terms of the types and amount of treatment that are affordable. Use of private clinics or hospitals by poorer people may sometimes depend on a conscious choice, but at other times is due to the inaccessibility of public services (Palmer, Mills, Wadee, Gilson, \& Schneider, 2003).

On the other hand, doubts have been expressed over the seemingly harmonious interface between private and public sectors. There is a view that profit-seeking in the private sector has potential for creating problems in the healthcare system (Hanson, et al., 2008). For example, in the British National Health Service (NHS) public hospitals treat private patients and vice versa. As a result, fears have been expressed that such a relationship could be a threat to the long-term sustainability of the NHS, as this could result in creeping privatisation and predatory behaviour among medical professionals (Powell \& Miller, 2014). Doctors who work both in the public and private sectors are faced with a conflict of interest, in the sense that personal gains may not equate with benefits to the public system in which the professionals work. It is therefore, important to examine in detail the effects of DP in terms of the services rendered to both public and private consumers. This should be a central policy question for any health system where this practice exists.

The wider space occupied by the private health sector in developing countries, including Nigeria may suggest that it is lucrative, and complementary to public service. However, it has been argued that the private health care market has failed for two obvious reasons (Hanson, et al., 2008). First, private health care services do not seek to provide key public services that have external benefits, such as prevention of communicable diseases; they do not seem to 
value these benefits as saleable products in the health market. Secondly, patients' lack of technical knowledge, and the role of provider-directed care may leave them receiving low quality treatments (Hanson et. al., 2008). Thus, Pietro (2006) argues that private hospitals could concentrate on minor services not contained in the major services being carried out by the public healthcare system. This arrangement could give the more substantial healthcare delivery roles to the public sector, while complementing this provision with peripheral private providers.

Nevertheless, in weak public healthcare systems there is the potential for the private sector to thrive due to a lack of fundamental resources and infrastructure for efficient healthcare provision in the public sector. If the dual practitioner is a self-interested opportunist, his/her allegiance to public practice remains weak, and how he or she resolves the conflict of interest arising from working in both sectors is crucially relevant to understanding if the balance is more to either of the sectors. Although, the role of the private sector in healthcare provision remains important, the pertinent policy question could be how to regulate it to avoid potential threats to the public sector.

The situation may be more complex in developing countries, including Nigeria, where private practice may be restricted to off-duty hours, but there is no control over a top-up income. The off-duty hour restriction requires a public doctor not to engage in private practice during the official duty hour contract. A UK study that investigated time spent in the private sector by public-sector doctors found that consultants on average set aside two and a half days weekly for private consultations and operations (Yates, 2000). In reality, some doctors in the public sector operate full-time private practice and spend little time at government health facilities. This results in absenteeism by public doctors, which gives rise to doctor-hours and productivity losses (Isah, Omorogbe, Orji, \& Oyovwe, 2008).

The in-built administrative mechanism for monitoring doctors' performance in the public system is weak. The remuneration system, based on standard salary scales, does not create incentives for good efficiency, performance, workload, quality or health outcomes, and this also could encourage private practice. The official hours restriction (public-sector doctors not permitted to attend to private patients during the official hours contract in the public sector), if 
not monitored could give rise to unintended consequences such as reduced attention to public patients and giving more time to private practice because of the profit motive and allegiance to private patients.

Our main focus in this study is on dual practice among physicians employed in the public sector, who are on salary, but run private practices reimbursed via fee-for-service. In high, low-and-medium-income countries dual practice is common (Eggleston \& Bir, 2006; Hipgrave \& Hort, 2013; Jean \& Sass, 1995), but different forms of dual practice exist. One scenario is 'public-on-public' practice where the rules permit a physician to work in two different public hospitals. This is evident in Canadian healthcare (Hamilton, Letourneau, Pekeles, Voaklander, \& Johnston, 1997). A second scenario is 'private-on-private' dual practice, where a physician is allowed to run two private practices at the same time. This is most commonly found in the USA (Garcia-Prado \& Gonzalez, 2011). It is also evident in Nigeria among doctors in the private sector who work in two or more private hospitals on different shifts.

The most common form is 'public-on-private' dual practice, where physicians work in both public and private sectors, i.e. by holding a full-time post in the public sector, and also working part-time in the private sector. This type of DP has different variants. One of these permits physicians to run a private practice in the same public hospital where they work. This is evident in France, Germany, Italy, Ireland, and other European countries (Rickman \& McGuire, 1999). In this type, public doctors can earn extra fees by treating private patients in the public hospital where they work. This is currently being proposed in tertiary hospitals in Nigeria. Dual practice also exists within public facilities outside the contracted public sector hours using public resources (Russo, McPake, Fronteira, \& Ferrinho, 2013). Patients seek these services to avoid long wait-times in public facilities at a higher fee, and with a fixed amount passed on to the hospital. Moreover, private patients may prefer such services if the quality is proved to be better than that of the public service, but often not.

Another important variant is when physicians on full-time public-sector contracts combine this work with private practice, usually outside the public hospital. This is common in both developed and developing countries (Garcia-Prado \& Gonzalez, 2011) including Nigeria, i.e. 
the physician regards the public-sector post as the primary job but engages in private practice where similar clinical work is performed in return for extra private fees (Russo, McPake, Fronteira, \& Ferrinho, 2013).

In Nigeria, 'public-on-private' practice is common among health workers, particularly medical doctors. The engagement in additional income generating activities helps the practitioner to top-up their income. Available evidence shows that in 2001, 30\% of public medical officers were engaged in clinical work as income supplementation activity, while 10\% were involved in private home health services (McCoy et al., 2008). Since 2001, the trend has increased tremendously. It was found that $68 \%$ of public doctors make more money from supplementary work than from salary, while $75 \%$ would give priority to jobs that would enable them to earn fees for service (Akwataghibe, Samaranayake, Lemiere, \& Dieleman, 2013). The increase in income supplementation activities among public doctors may be a coping strategy to make up for their supposedly low salary.

The Nigerian public health sector is characterized by frequent absences and late reporting for duty. This is often attributed to private practice where medical practitioners choose to see their private patients before reporting at the public facility. The bureaucratic/administrative rules of attendance are often ignored with impunity. Although, guidelines allow private practice only within off-duty hours, this rule is rarely adhered to, leaving public patients to experience increased waiting times (Olowookere, Fatiregun, Ladipo, \& Akenova, 2011; Umar, Ochem, \& Umar, 2011). In peri-urban and rural areas, full-time public doctors are often available at health facilities only on designated days, while the other days may be spent on private practice or other income-generating activities.

In 2010, Osuagu published a paper on Ethics and medico legal aspects of medical practice in which he decried the situation whereby many public-sector doctors on full-time contract operate a 24-hour private hospital service and work in their spare time in government hospitals. The author narrated his experience as a medical student to show that some consultants would report for work after 11 am on the few days they choose to come in at all (Osuagu, 2010). A similar whistle-blowing example can be found in a practicing doctor's memo "Regulation of Private Practice by Government-Employed Medical and Dental 
Practitioners" to MDCN dated 23 ${ }^{\text {rd }}$ September 2011 (Iregbu, 2011). This doctor challenges his professional colleagues and argues that it is morally reprehensible to compete for business with their employer - the government. He further contends that, despite the DP policy in Nigeria, many medical and dental practitioners have continued to establish huge and sophisticated medical businesses that operate a 24 hours service, thus using their employer's time to run a private business.

Consequently, patient diversion from government hospital to private hospital seems to have become commonplace as doctors routinely point out shortcomings in government hospitals to patients in order to justify the diversion (Iregbu, 2011). With the above in mind there is a clear need to generate evidence on the implications of dual practice for the Nigerian healthcare system.

Past studies of dual practice have looked narrowly at the issue, and often overlooked the importance of context and the structure of the healthcare market in the healthcare systems studied. Similarly, investigation of the motives for dual practice has often focused on the dual practitioners alone. Several studies in the past did not interview non-dual practice physicians in the public hospitals to ascertain why they chose to work exclusively in the public sector (Abera, et al., 2017; Ashmore, 2013; Ferrinho, et al., 1998; Humphrey \& Russell, 2004; Ligia, 2014; McPake, Russo, \& Tseng, 2014; Zhang, 2015), but it is important to establish the reasoning of doctors who commit exclusively to public sector work in a context where the majority of colleagues engage in dual practice. Most of the arguments about DP concern social values, individual motivations and multiple impacts on the healthcare system, and the use of econometric analysis without sufficient empirical data on these matters provides an incomplete picture (Berman \& Cuizon, 2004; Biglaiser \& Ma, 2007; Eggleston \& Bir, 2006; Gonzalez, 2004; González \& Macho-Stadler, 2013; Hipgrave \& Hort, 2013). DP has rarely been studied using a mixed-methods approach, but this is crucial to develop a more complete understanding of the problem. In the Nigerian context, there is a pressing need for research to underpin the current policy debate on DP and inform possible regulatory changes that are presently being considered. 
This study attempts to fill these gaps by investigating the benefits and disbenefits of dual practice via an exploration of the perspectives of service users, policy makers/stakeholders and dual practitioners working in the public system. The value of hours lost to the public system due to absences and reporting late for work by dual practice physicians was estimated for both normal routine and on-call duties. On the side of the service users, information was collected to understand whether there was an additional cost for services that could have been obtained in the public sector but were obtained in the dual physician's private clinic due to patient diversion. The study also determined the extent of self-referral (patient diversion) among dual practice physicians, and the gender, age group and socio-economic status of the diverted patients.

The establishment of these facts is vitally important and will help to generate insights regarding the effects of dual practice in the healthcare system and what regulatory mechanism may best address the problem. Additionally, this study sets out to compare the quality of healthcare provision in the public sector setting where the dual practitioner works, with quality of services in their private practice. The aim was to investigate the difference, if any, and to explore the possible factors that account for such differences. Furthermore, it was important to explore dual practitioners' motives for engaging in dual practice and the motives for not engaging in dual practice among the non-dual practice doctors, to understand the economic and social factors that shaped behaviour.

\subsection{Structure of Nigerian healthcare system}

The Nigerian healthcare system is structured according to the three levels of primary, secondary and tertiary services. Primary health care (PHC) serves as the point of entry to the care process. This includes clinics and health centres where consultation and out-patient treatment services take place, as well as health promotion and disease prevention programmes in areas such as maternal and child care, family planning, and immunization. A few bed spaces for observation may also be available (MDCN, 2008), but patients with more serious conditions will generally be referred to secondary care facilities. Primary health care has largely collapsed in Nigeria and can best be described as the first entry point for the poor who lack the money to arrange early access to a higher level of care. 
The secondary level of care comprises general hospitals and medical centres that serve as destinations for referrals from the primary care facilities. In theory, these provide more specialised services with more skilled personnel. The type of services they offer may differ across locations but essentially, they provide inpatient and outpatient services for specialities such as general medicine, general surgery, paediatrics, obstetrics and gynaecology as well as some community health services. However, referral from primary to secondary is not linear, patients can move from primary to tertiary without visiting the secondary level. This level of care may not have adequate resources to cater for the needs of the local community, and transfers to better equipped hospitals may be necessary.

Tertiary health care is made up of highly specialized services provided by the teaching hospitals, specialty hospitals and federal medical centres. They provide care for specific disease conditions or specific groups of patients. This level has the most specialized medical personnel and more advanced medical technologies that enable it to provide more effective health care services. The institutions at this level are the referral hospitals from lower-level health care providers, except for accident and emergency care that must necessarily be provided locally (MDCN, 2008). In practice, patients may come directly to tertiary-level hospitals without referral from other health facilities.

The three levels of services lack communication and each often seems to be independent of the other, which makes it difficult for the secondary and primary levels to function as gatekeepers to the tertiary level.

\subsection{Nigerian healthcare system}

A healthcare system, according to the World Health Organization, includes "all the activities whose primary intent is to promote, restore, or maintain health" (kruk \& Freedman, 2008; WHO, 2007). The emergence of a modern healthcare system in Nigeria is traceable to the colonial period (Alubo, 2001). It was noted that the initial medical service provision was meant for the Europeans and their staff, so as to maintain a healthy labour force, but was later extended to the general populace as largesse. Health service provision was curative, urbanbased and elitist in nature to the neglect of the rural population. This skewed pattern of health 
care access is still part of Nigerian healthcare service provision (Ichoku, Fonta, \& Ataguba, 2013). In the colonial period, the organization of formal health care services took a tripartite form, comprising public, private and mission/voluntary agencies. The growth of mission health care facilities at that time complemented the role of the public healthcare sector.

After independence in 1960, there was an obvious growth in the public healthcare sector, which resulted in an increase in both human and infrastructural capacity. The number of medical training institutes increased tremendously with a growing number of physicians (Scott-Emuakpor, 2010). This growth in human resources and

infrastructure was made possible by the oil boom of the early 1970s, and the reverse seemed to be the case in the late 1980s when a financial crisis impacted on government revenues and spending (Alubo, 2001).

The economic crisis that engulfed Nigeria in the early 1980s was accentuated by the Structural Adjustment Programme (SAP) associated with the neoliberal economic policies pursued at that time by the International Monetary Fund (IMF) and the World Bank (WB) (Orubuloye \& Oni, 1996). The negative impact of this policy is still being felt in the health sector at present. During the implementation of the SAP, resource allocation to the healthcare sector dwindled significantly (Orubuloye, 1996). The consequences of the implementation of the SAP included downsizing of the health workforce, and rising costs of health care services as a result of the introduction of fee-for-service in public healthcare facilities. Access to government hospitals was limited due to the rising cost of medication, as well as shortages of medicines and medical equipment. As a result, there was a public loss of trust and confidence in the public healthcare sector. This encouraged an increase in private medical enterprises in Nigeria, despite the cost to patients.

Thirty years or so of military rule in Nigeria has had a considerable negative impact on the healthcare system. Evidence has shown that the long period of military presence in the Nigerian polity restricted access to public healthcare facilities, and led to downsizing of the health work force and a brain drain of medical professionals (Alubo, 1992). The agreement to IMF conditionalities to secure a US $\$ 2.5$ billion-dollar IMF loan, despite a public outcry against it, was secretly taken by the military regime. The implementation of these conditions, 
apart from precipitating social and economic crises, resulted in the deterioration of the healthcare system. One outcome was worsening coverage with only $35 \%$ of the population having access to modern health care services (Alubo, 1992). Under the military the budget allocated to public healthcare contracted to $2.7 \%$ of public expenditure on health (ScottEmuakpor, 2010).

\subsection{Performance of Nigerian healthcare system}

The Nigerian healthcare system is noted to be weak in many aspects, ranking 187 out of 191 country health systems assessed in the last global WHO survey (WHO, 2000). It has performed poorly on Nigeria's national health indicators (Kombe et al., 2009). For instance, the under-five mortality rate was 108 per 1000 live births in 2015 (UNDP, 2016), and the most recent maternal mortality ratio is 596 per 100,000 live births, higher than the SubSaharan Africa average of 511 deaths (Izugbara \& Wekesah, 2017). The rising disease burden is also worrisome because it further stretches the healthcare system's available resources. The prevalence of HIV/AIDS is 3.2\% (Awofala \& Ogundele, 2016). The challenge of the burden of non-communicable diseases is also a concern in Nigeria (Musa \& Musa, 2014).

Public healthcare financing in Nigeria comes from different sources. This includes tax revenue, donor funding, out-of-pocket payments, and social insurance. The National Health Insurance Scheme was introduced under Act 35 of 1999 Constitution with the objectives of improving access to health care and removing the heavy financial burden created by out-ofpocket payments. The scheme currently, covers only federal government employees and up to four dependants (Mohammed, Souares, Bermejo, Sauerborn, \& Dong, 2014), leaving behind the majority in the informal sector without coverage. Despite the high coverage achieved by the scheme for those in the public sector (Obikeze et al., 2013); the only contribution to equity is that the government subsidizes the scheme for those in the informal sector who ordinarily would not be able to afford contributions. Thus, there is much work to be done to achieve improved access to health care for all Nigerians. Furthermore, it has been shown that relative to the high burden of disease and population, healthcare financing in Nigeria remains low both from state and federal budgets (Kombe et al., 2009). 
The public health system has suffered a series of crises. Total expenditure on health as a percentage of the Gross Domestic Product (GDP) was already low at 3.3\% in 2012 (World Data Atlas, 2012 ) and slightly increased to 3.7\% in 2014, with just $0.9 \%$ being government spending and one of the lowest public spends in Africa (WHO, 2016). Low state expenditure leads to a heavy reliance on out-of-pocket spending by the poor that constitutes a financial barrier to access, and results in further impoverishment. For instance, private expenditure on health as a percentage of total expenditure on health in 2014 was $74.9 \%$ (WHO, 2016). The total budget allocated to health remains abysmally low at less than $4 \%$ of the annual budget in 2018 (Onyeji, 2017), far below the 15\% recommended by the African Union (AU) in 2001 "Abuja Declaration".

Industrial action by medical professionals and other categories of public health care providers is a common occurrence in the Nigerian public healthcare sector. The incessant strike actions by public doctors in Nigeria have led to the closure of both secondary and tertiary health care facilities in the country for many weeks (Akinyemi \& Atilola, 2012), thereby posing a potential threat to the healthcare system. During strike periods, public hospitals are shut down completely with the inpatients abandoned and put at risk. The medical profession has been accused of selfish demands from the government, and as a result has contributed to avoidable deaths resulting from strike actions. It is argued in this regard that the medical profession is a privileged group with its own vested interests, which the group strives to protect (Alubo, 1986).

As these things have happened, a new crisis of professional rivalry characterised by distrust, dissension and power tussle over the control of the health sector between the medical professionals and other professional groups has emerged in the Nigerian health sector (Adeloye et al., 2017). It has been alleged by other professional groups that the health sector has been under the hegemony of the medical profession and there ought to be a change of baton (Alubo \& Hunduh, 2017). The areas of contention include the backgrounds of those appointed as Minister of Health or senior Ministry consultants, as well as heading the various government health institutions. All these areas, according to other professional groups, have been under the control of medical doctors. This overtly unhealthy relationship between the 
medical profession and other professional groups in the health sector poses a great risk to both patient welfare and health facility management.

Healthcare system governance involves accountability in the use of public resources, transparency, how government policies take on meaning for the citizens, and evolving a system in which professionals, managers, service users, the public all have some participation (Brinkerhoff \& Bossert, 2008). The health system governance structure in Nigeria is weak, and characterised by poor management, corruption, lack of accountability and poor healthcare leadership (Adeloye, et al., 2017). The measures of governance, such as voice and accountability, political stability, rule of law, government effectiveness, regulatory quality, and control of corruption (Kaufmann, Kraay, \& Mastruzzi, 2006), pose serious challenges to health system governance in Nigeria. For instance, Kamorudeen \& Abdulkareem, (2012) showed how the Nigerian Federal Ministry of Health stole a large sum of money from the Millennium Development Fund. It was further alleged that the same Ministry was involved in inflating the cost of purchasing HIV/AIDS drugs for HIV-infected persons in the country. The formulation of health policies is mostly top down. Although, new policies are instituted at both state and federal levels, these are often implemented unevenly. The manner in which health policies are formulated often lacks an evidence base, and successful implementation is mostly difficult to achieve (McKenzie, Sokpo, \& Ager, 2014). Despite sound frameworks for the development of national health policies, the inputs from consultations were hardly used in developing policies.

There is inequality in the distribution of health care services between the urban and rural areas with a concentration of secondary and tertiary care in the urban areas, which have relatively better medical equipment, technologies and supplies than the rural areas (Adeloye, et al., 2017; Ogunbekun, Ogunbekun, \& Orobaton, 1999). The same pattern is discernible in the siting of diagnostic centres (Alubo, 2001). The current social health insurance system in Nigeria covers only federal government employees; it is of no assistance to most rural dwellers and there is no alternative financial protection mechanism to cushion the effect of catastrophic payments in the event of ill health for this group. 
Similarly, rural areas have fewer highly qualified health workers than the urban areas. As a result, the health indicators in rural areas show worse outcomes. For example, in rural areas there is a high under-five mortality rate of 145 per 1000 live births, compared with 79 per 1000 live births in urban areas (Morakinyo \& Fagbamigbe, 2017), and more number of deliveries by traditional birth attendants (TBAs) at home than in PHCs due to demand side factors (Fapohunda \& Orobaton, 2013).

The participation of civil society organizations in projecting and protecting patients' voices and ensuring that providers and policy makers are held accountable is weak and often nonexistent. There is scant evidence about the effectiveness of consumer protection organizations in Nigeria. Civil society is rarely involved in policy formulation.

\subsection{The private sector}

The private sector plays a very important role in the provision of care and has a wide range of providers (Kombe et al. 2009; Pietro, 2006). In many developed countries, the private sector has been viewed as complementary to publicly controlled or regulated national health service or social health insurance systems (Doyle \& Bull, 2000). A vast majority of health care services in low-and-middle income countries are offered by the private sector (Mills, Brugha, Hanson, \& McPake, 2002). There is an argument that work in the private sector is rewarding, and many general practitioners are drawn to the sector for the incentives it offers (Palmer, et al., 2003).

In Nigeria, private medical practice has a long history. The activities of early church missionaries in the provision of medical services in Nigeria were significant. When colonial governments failed to make adequate health care provision, it was the missionaries who were in the forefront of providing care to the people (Diara \& Nche, 2013). There were well-known hospitals established by the missionaries all over Nigeria. The mortality rates associated with diseases such as malaria, sleeping sickness, dysentery and measles fell sharply as a result of these endeavours. Most of these mission hospitals are still functioning, and have made a huge impact that complements the public healthcare sector in Nigeria 
The crisis in the Nigerian public healthcare sector seems to be a catalyst for the growth of private healthcare, which is developing at both formal and informal levels. The formal level generally involves enterprises that are registered with the government and have a designated place of operation, but the informal level is itinerant and less organized (Alubo, 2001). There has been a proliferation of private-for-profit healthcare facilities in Nigeria since the 1980s (Ogunbekun, et al., 1999), a period marked by economic recession. Private practice in Nigeria is mostly solo practice, whether by qualified staff in offices, maternity homes and pharmacy shops, or by patent medicine dealers and itinerant drug peddlers.

Despite the benefits of private medical practice in Nigeria, the private sector still suffers from many shortcomings such as indiscriminate administration of injections, inappropriate pharmaceuticals supplied by drug peddlers, fake drugs, and so on (Alubo, 2001). It has also been argued that the high fees charged in the private sector limit access to private medical care among the poor (Onwujekwe et al., 2010). The high fees charged by private providers drive people who cannot afford the cost to seek health care services in the informal private sector where all kinds of untested herbal and traditional drugs are sold. Government regulations have not been able to control these practices.

The private health care facilities are often less well equipped than state facilities, but notwithstanding, they are much sought after due to the critical role they play in making health care treatment accessible to the people. They have gained the trust and confidence of the people as a result of their widespread availability, and the credit facilities they offer - a situation where patients who would find it difficult to make a one-off payment for treatment cost are allowed to pay in instalments (Onah \& Govender, 2014). This rarely happens in the public sector. There have been suggestions that private facilities have advantages in terms of greater responsiveness, shorter waiting times, and better quality (Adesanya et al., 2012), but this remains a matter of controversy and has not been established via systematic research. Indeed, there is a general lack of information on the activities of the private sector in Nigeria, and its quality and utilization rates, and there has been a failure to apply methodologies such as impact evaluation which would help build evidence-based policy (SHOP Project, 2016). 
The relationship between the public and private health sectors does not appear to be complementary in the sense of a cooperative relationship between the two. There is limited interaction between public and private health institutions (Ogunbekun, et al., 1999). The National Policy on Public-Private Partnerships (PPPs) for health in Nigeria was initiated in 2005. The aim was to strengthen the health sector through public/private cooperation. It was obvious that the inadequate resources allocated to health, high infant and maternal mortality rates, poor service delivery at all levels, and the rising disease burden in the country, were some of the major concerns that drove the government to consider partnership with the private sector (Federal Ministry of Health, 2005). However, there has not been a standardized framework for the implementation of PPPs in Nigeria. In addition, over 50\% of the public health care workforce does not support the PPPs policy (Anyaehie et al., 2014), which suggests that they may seek to frustrate its implementation. The referral system is poor, mainly from the private to public, which also shows that the two sectors do not complement each other well (Ogunbekun, et al., 1999). The pertinent question, therefore, is whether the government has the capacity to implement PPPs and monitor the private sector. Moreover, the capacity of the state and other key stakeholders seem insufficient to tackle the regulatory challenges confronting the health market (Siddiqi et al., 2009). As a result, many transactions in the health market happen outside the official regulatory framework in Nigeria.

\subsection{Medical regulation in Nigeria}

The Medical and Dental Council of Nigeria (MDCN) is the statutory body set up by law under the Act (Medical and Dental Practitioners Act Cap 221 [now Cap M8] Laws of the Federation of Nigeria 1990 and amended by the Decree No 79 of 1992) (Vries et al., 2009). Under the Act the MDCN is charged with the responsibility of regulating medical practice in Nigeria. The MDCN determines the standards, knowledge and skills to be attained by persons seeking to become members of the medical and dental professions and reviewing those standards from time to time as circumstances may permit. It also decides who is qualified and admits them into the profession and keeps a list of registered professionals and oversees the practice of medicine. The Council has a 61-member body known as the Council, on which each state of the federation is represented by its director of medical services. 
The Council establishes a State Monitoring Committee at the state level chaired by the director of medical services. The Chairman of the Nigerian Medical Association (NMA) or its representative is a member of the committee. The committee is charged with the responsibility of monitoring doctors at facilities in the state.

It is important to clarify that MDCN is an agency under the Federal Ministry of Health. Therefore, the MDCN does not have the statutory power to employ doctors; instead, doctors are employed at federal, state and local government levels. MDCN in this regard, does not interfere in employer/employee relations. However, if the employer reports an employee for any practice-related offences, the matter is received by the Council's Professional Disciplinary Department and is further investigated and tried before the Disciplinary Tribunal. If the professional in question is found guilty, they would be sanctioned appropriately depending on the severity of offence. Three levels of punishments are available for the Tribunal: the admonishment of the practitioner depending on the nature of misconduct; suspending the practitioner from medical practice for a period not to exceed six months; and finally striking off the practitioner from the professional register. A member of the public can also file a complaint against a doctor through the same channel and in affidavit form.

On the other hand, it is not clear what misconduct would deserve any of the punishments specified by the MDCN's Disciplinary Panel. The findings of the panel might more often favour medical professionals. For instance, the disciplinary procedures do not provide for compensation to the patient who may be a victim of medical malpractice. The MDCN and NMA, taken together, have power to define what constitutes professional misconduct. Patients and the public need clear information about their rights and when those rights have been violated during contacts with clinicians, but this is rarely forthcoming. The profession's image in the eyes of the public may be at stake if the transparency of the existing mechanisms of self-regulation is not improved.

Furthermore, the use of the Tribunal Court instead of the regular court confers power to the medical profession without due consideration of the victims of professional misconduct. In German Medical regulation, for instance, the Chamber of Doctors, which is comparable with the NMA in Nigeria, can run arbitration/meditation procedures to facilitate out-of-court 
settlements between patients and doctors (Vries et al., 2009). There are no arrangements of this kind in Nigeria. Therefore, transparency in the Council's court trials needs to be enhanced.

Moreover, MDCN is faced with some challenges that potentially affect the discharge of its role as the regulator of medical practice. The lack of funding to carry out its statutory responsibilities is one of the biggest challenges. Yet its financial contribution to the monitoring committees at the state level is not enough even to pay for vehicles for monitoring activities. The lack of money further hampers visits to rural areas where quackery may be common. The connection between the Council, Nigeria Medical Association and the Directorate of Medical Service (DMS) lacks a clear structure of accountability. The DMS depends on MDCN funding to monitor facilities at state level, but this is most often not forthcoming. The NMA does not have much stake in medical practice regulation in Nigeria. Apart from being an interest group with the sole purpose of protecting the interest of its members, it does not have statutory power or derived from MDCN to be in the fore front of medical regulation. Furthermore, how the NMA and state collaborate in the regulation of medical professionals needs proper clarification so that the parties know who does what and who should be held accountable for what.

NMA, on the other hand, is an umbrella body of all medical practitioners in Nigeria and can be described as a sectional interest group that bargains with the government on matters affecting the interest of its members. It has a strong influence in shaping health policy in Nigeria. It has used its influence to negotiate salary increases, and to maintain a dominant position for its members in the administration of government hospitals in Nigeria. For instance, only a medical doctor can head a public healthcare facility in Nigeria, including the primary health centres. The organization has on several occasions embarked on industrial action to make one demand or the other from the government. In some cases, it could be a protest against a proposed government reform initiative, which the NMA considers not to be in the best interest of the profession. Other disputes have been about remuneration, as for example, when the Lagos State Government sacked nearly 800 public doctors for embarking on an indefinite strike over pay demands (Odigwe, 2012). During the strike, there was a total withdrawal of services by the striking doctors leaving the nurses to provide emergency 
services. This may suggest that patient-centeredness could be difficult to achieve in such an environment.

The current practice where only the medical profession has the sole authority to monitor the market for health care seems to invite the risk of a cover-up of members' misconduct and could also lead to ineffective regulation of the healthcare market in Nigeria. It has been noted that self-regulation, although sometimes presented as being in the public interest, often has the goal of protecting professional interests (Blair \& Rubin, 1980).

The top-down approach of medical practice regulation in Nigeria does not seem to provide a balanced system that brings service users on board. For instance, the MDCN relies on the employer to report malpractice-related offences to the Council but has no channel through which complaints from service users can move from the lower level to the higher level. There is a lack of any arrangement at the lower level whereby complaints can be collated from the voiceless poor who may be victims of medical malpractice. In a context where laws are abused, there is a possibility of receiving a low number of reports of medical malpractice, which may send a misleading signal about the real situation.

There is an obvious absence of consumer participation in the regulation of medical practice in Nigeria. In the UK for instance, bodies like 'Healthwatch' (England) and Community Health Councils (Wales) offer mechanisms for patient complaints and the expression of public voice. Patient groups have helped shape treatment guidelines developed by the National Institute for Clinical Excellence (NICE) and influencing other key health policy decisions (Farrel, 2004).

The level of consumer participation in shaping health policy in Nigeria is poor both at the national and state levels (Kombe, et al., 2009). There is no effective channel to allow service users to participate in clinical decisions affecting them, to influence health technology appraisal, or even to report any threat to, or violation of their rights by health care providers. The absence of consumer groups in Nigeria is an indication that patients have no voice in the regulation of medical practice. This may result in regulatory capture by the medical profession because it could intentionally avoid publicizing medical malpractice for the fear of damaging the reputation and standing of its members (Schuftan \& Unger, 2011). This raises the question of whether Nigeria needs consumer protection arrangements that will help secure 
redress for the victims of medical malpractice. It has been suggested that establishing a proper channel for patients' complaints could increase quality (Gladstone, 2000).

Additionally, price regulation of private health care is not based on any single methodology or a unified costing system. Hospitals and clinics in Nigeria charge patients at their own discretion (Ogunbekun, et al., 1999) and emergency cases are rarely treated without a cash deposit, even at the point of death. The Private Hospitals (Regulation of Standards) Bill 2009, which is meant to define the framework for charging in the private health sector in Nigeria, has not been signed into law. At present, the pricing system in the private sector is patterned after other commercial activities.

The market for private healthcare in Nigeria seems poorly regulated. It could be that the market is too large, with ineffective regulatory agencies and inadequate resources to carry out effective monitoring of providers' activities. As a result, it is difficult to oversee the activities of medical professionals due to the large and diverse private sector.

The Nigerian healthcare system has a long history going back to the colonial period, through military rule and then democratic governments. Each of these epochs was characterized by inertia and limited progress. As a result, Nigeria failed to introduce a public health system that is efficient, responsive and accessible. The growth of the private health sector may have stemmed from the failure of the public system. The weaknesses of the governance system and the ineffectiveness of the regulatory mechanisms have further contributed to the poor health indicators achieved. There is need for policies that spell out the ground rules for public and private actors to work alongside each other.

\subsection{The Code of Medical Ethics in Nigeria (formerly Rules of Professional Conduct for Medical and Dental Practitioners in Nigeria)}

The MDCN has the responsibility to prepare periodically a statement on the Code of Conduct which the Council considers appropriate for the practice of medicine in Nigeria. This statement was formally known as the Rules of Professional Conduct of Medical and Dental Practitioners in Nigeria, which was first published in 1963. In 2004, a new edition of the statement was published, and the title was changed to Code of Medical Ethics in Nigeria 
(Vries, et al., 2009). The impact of the change in nomenclature is not obvious, although, the aim is to ensure that all medical practitioners familiarize themselves with the contents of the code, which covers key issues relating to medical practice, biomedical and other forms of medical research. The Code (MDCN, 2008) holds that medical and dental surgeons in fulltime employment in the public sector are free to use their off-duty hours to engage in private medical or dental practice for remuneration under the following conditions:

A registered practitioner on full-time public employment shall not engage in private practice during official hours under any circumstance. A registered practitioner who holds the appointment of a consultant or a medical or dental officer for ten years post registration may be eligible to run a private consulting clinic that will open for business only when not on official duty. A consultant or a registered practitioner of similar status as described in the code shall offer in-hospital care to his private patients only within the public hospital where he is on full employment. It is unethical, therefore, for a registered practitioner on full-time public employment to give in-hospital care, which is investigatory, admission, and institution-based care to patients outside the hospital where he is on full employment. A registered practitioner of more than ten years post-registration who is on full-time in the public sector, but not engaged in clinical responsibilities in the public hospital may only engage in clinical practice outside his official hours in an institution owned and managed by a full-time private practitioner or hold consultations only in his own consulting clinic.

The self-regulatory function of MDCN and NMA places with these organisations the responsibility for monitoring and sanctioning medical professionals. But what seems to be challenging is how far in reality the profession can regulate itself. This is evident in the obvious flouting of the Code of Medical Ethics in Nigeria as it relates to private practice by public-sector doctors.

\subsection{Constitutional provisions regarding private practice in Nigeria}

In 1984, during the Military era, the ruling Junta promulgated Decree 34, under the Regulated and other Professions (Private Practice Prohibition) Act, CAP. 390 (Special Military Tribunal Act, 1984), which prohibits private practice by professionals in the public service. The Act 
states that a "public officer shall not have his personal interest conflict with his official duties and responsibilities" (Special Military Tribunal Act, 1984). The Decree defines a public officer as any person who holds office in the public service of the Federation or a State within the meaning of the Constitution of the Federal Republic of Nigeria. The definition imposed by the Decree on what constitutes private practice is relevant to the subject of dual practice. The Decree describes private practice as including "rendering of or offer to render to any other person (not being the employer or any other person normally entitled in the course of his official duties to receive such services) of any services relative to the professional concerned whether or not after his normal hours of work or on work free days, in cash or in kind, or for any other valuable consideration" (Special Military Tribunal Act, 1984).

Notwithstanding the above, the Decree allows that it is lawful for the professional to render services within official duty hours to himself /herself or any person in an emergency, or to any other person from a corporate institution, which the government has to authorize to receive the services of that professional for a given duration. In addition, services may also be rendered to any member of the professional's family, any professional association to which he /she belongs, and any charitable organization without profit motive. The Decree further spells out various penalties for those who contravene this law. It stipulates that a first offender under the Decree shall pay a fine of two thousand naira $(£ 4)$, or one year of imprisonment. A second offender is liable to a fine of five thousand naira ( $£ 10)$, or two years of imprisonment; and a third offender to three years of imprisonment without any option of fine. There are, however, no data available at the present time on the number of offenders punished under this Decree.

In response to this Decree, there was opposition from some public officers. The Military Government also witnessed a brain drain caused by the Decree, and decided to loosen the rule for some categories of public sector professionals (Badejogbin, 2007). The outcome of the agitation was the Regulated and Other Professions (Private Practice Prohibition) (Law Lecturers' Exemption) (No. 2) Order, which exempted law lecturers, engaged in the practice of law.

Later, when the Constitution of the Federal Republic of Nigeria came into force on May $29^{\text {th }}$, 1999, the earlier Decree prohibiting private practice by professionals was consequently 
repealed under the Certain Consequential Repeals Decree No. 63 of 1999. The Constitution, therefore, imposes its supremacy over any other existing laws and Decrees permitting private practice among professionals in the public sector. Nevertheless, the Constitution in its fifth schedule under the Code of Conduct for Public Officers maintained that a public officer shall not have a conflict of interest where his personal interest conflicts with his duties and responsibilities (Federal Republic of Nigeria, 1999). It is therefore the rules/codes for professional conduct that in practice regulate doctors' dual practice rather than the legislation.

\subsection{Research aims and objectives}

\subsubsection{Aim of the study}

This study investigates dual practice by medical professionals working in public healthcare facilities in Nigeria, and motivations for and attitudes towards it. It attempts to generate evidence to inform policy on the effects of DP in the health sector. This was achieved by addressing a number of more specific research questions.

\subsubsection{Study research questions}

The study seeks to answer the following questions.

1. What are the benefits and disbenefits of dual practice in the health sector in Nigeria?

2. What is the extent of physician self-referral in public hospitals in Nigeria?

3. How does the perceived quality of healthcare in the public sector compare with that of healthcare in private healthcare facilities managed by dual practitioners?

4. What are the motives that lead Nigerian doctors to engage in dual practice, or refrain from doing so?

5. What are stakeholders' views on the implementation of existing regulations on DP, alternative regulatory mechanisms/options for controlling dual practice and the challenges for reform? 


\section{Methods and methodology}

This chapter discusses the methodological choices made in the study and the methods employed. First the choice of study location is described. The chapter then considers certain epistemological issues arising in social research, and outlines the stance taken in the mixedmethods approach adopted. The component methods that addressed the objectives of the study are described, and the approach taken to data analysis, rigour and ethics are examined. Finally, the methods used in the literature review are explained.

\subsection{Study location}

The study took place in Enugu State, South-east Nigeria. In 1928, Enugu became the headquarters city of the southern province, and today is the State capital. There are seventeen local government areas (LGAs). The state has an estimated population of 3,257,298 (National Population Commission, 2006). There are 488 public health facilities and 380 private health facilities (FMOH, 2011). The state is divided into seven health districts for the organisation of healthcare delivery services. Also, the state has a tertiary hospital where doctors are trained. The State Ministry of Health employs doctors and supervises the health facilities in the state. Besides the state-owned hospitals and clinics, there are federal government-owned public facilities operating in the state, but mainly at the tertiary level. The selection of the Enugu Urban area, containing three LGAs, as the main site for this study is due to the large concentration of private and public hospitals in the area, and to the fact that the majority of government doctors are located in the Enugu Urban area. As will be explained in more detail below, focus groups, and a household survey completed in the study included respondents from one LGA in the Enugu Urban area while the interview respondents were drawn from some tertiary hospitals in Enugu Urban. A few high-level informant interviews were done in Abuja where the Medical Council is located.

\subsection{Epistemological issues}

One of the foundational epistemological questions in social science concerns whether the social world can be studied in the same way as the natural world (Bryman, 2008). 
Epistemology is concerned with how we gain knowledge of the world around us. The contending theories of knowledge tend to centre on two opposed paradigms, the positivist and interpretivist.

Positivism is the application of the methods of natural sciences to both the natural and social domains. Such methods as applied to the social world generally involve a search for recurrent patterns of behaviour or associations between variables that hold across groups or populations and can be confirmed by such methods as quantitative surveys, cohort studies or experiments.

Interpretivism postulates that human actors are different from the natural world of inanimate objects, and therefore, should be studied differently. It argues that actors perceive the external world only indirectly through the filter of cultural knowledge and ways of seeing that are shaped in on-going social interaction and moment by moment 'interpretation' of unfolding events. The interpretivist argues that research needs to pay attention to social actors' own interpretation of their actions rather than seeing those actions as being determined by external reality (Bryman, 2008, Low 2013). Interpretivism is one of a family of approaches that examine the subjective perspectives of actors as well as the contexts in which action takes place. We have no space to study cognate approaches in this thesis, but reasons for focusing on interpretivism rather than rival perspectives, include the possibility of utilising it within a realist analysis (see below) and a mixed methods study.

Although some have presented positivism and interpretivism as opposed and irreconcilable approaches, others have argued that both the investigation of general patterns using methods similar to those of the natural sciences, and a distinctive social science approach that explores subjective perspectives, have a role to play. Thus, Murphy and associates (1998) argue that the quantitative method cannot claim superiority over the qualitative; instead, both can be seen as mutually enriching partners in a common enterprise. The immediate question that perplexes many is whether this makes sense in epistemological terms. Does interpretivism acknowledge the underlying reality that positivism seeks to describe? Does the former not rather point to multiple realities depending on varying individual interpretations of social situations? 
Hammersley (1989) attempts to find common ground via his concept of subtle realism. To counter the idea that qualitative studies inevitably lead the researcher towards multiple realities and relativism, he offers an interpretation of realist philosophy that provides space for both general social patterning and the subjective realm of individual perspectives. According to Hammersley, reality exists independently of the researcher's knowledge claims, but at the same time knowledge of phenomena is always gained from a particular social location and standpoint. Research reports cannot directly reproduce what is a highly complex and multifaceted reality but offer a series of representations that can never be wholly complete. However, although any reality can be represented from a range of different perspectives that do not mean that all claims are equally valid. Hammersley contends that some research reports make claims that are false and can be shown by evidence to be so. Thus, he argues that, although there is scope to represent reality from multiple angles, these representations are only likely to be valid to the extent that they are not contradictory. Accounts of social reality that directly compete cannot all be true, and in Hammersley's view the task of an empirical social science is to use evidence to see which accounts are most plausible. As in realist philosophy more generally, the underlying assumption is that human knowledge of reality is imperfect so that knowledge is always gained from a certain perspective, but that nevertheless the goal of science is to investigate that underlying reality as far as practically possible.

Several authors interested in applied qualitative or mixed-methods research have seen variants of realism as useful way forward e.g. (Maxwell \& Mittapalli, 2010; Mays \& Pope, 2000; Murphy, et al., 1998) accept that both quantitative and qualitative methods have a role, even though they each have distinctive goals. They contend that both types of research can be assessed against criteria of validity and relevance, but in different ways. Thus, they argue for the use of strategies that will improve the rigour of qualitative research, such as triangulation, respondent validation, clear exposition of data collection practices, reflexivity and attention to negative cases.

Certainly, qualitative methods associated with interpretivism, the in-depth interview and participant observation can complement findings from surveys or cohort studies. It has been pointed out that interpretivism has the potential to address important research questions that 
the positivist approach may not answer in health research (Green \& Thorogood, 2014; Pope \& Mays, 1995). Evidence from qualitative case studies has usefully supplemented survey findings on issues such as population need, the development of appropriate policy and how to implement healthcare policies (Green \& Thorogood, 2014). Despite differences in the two paradigms, it has been argued that a combination of methodological procedures from both could enrich a research output (Bryman, 2008).

\subsection{Mixed-methods study}

A mixed-method study is described as an approach to research in social, behavioural or health sciences, which offers the researcher the opportunity to collect information using both quantitative and qualitative data and then draws interpretation on the strength of both sets of data to understand the research problem (Creswell, 2015). By this combination, the weaknesses of a single method may be eliminated thereby synergizing their strengths for more trustworthy results. Green \& Thorogood (2014) have shown that data integration from different sources can add breadth and depth or strengthen the validity of the data. As argued above, qualitative and quantitative methods can be seen as complementary and mutually reinforcing (Murphy, 1998). In this study, therefore, both qualitative and quantitative methods were used to examine the research problem using a triangulated approach. The qualitative approach is associated with interviews, focus group discussions, participant observation, ethnography, life history, case studies etc., while quantitative methods include surveys, experimental studies, cohort studies, randomised controlled trials and so on. In this study, the qualitative methods used were in-depth interviews and focus group discussions, and the quantitative components comprised a ranking instrument administered at the time of doctors' interviews and a household survey. These methods were selected because they were judged to be appropriate for addressing the chosen research questions.

Overall, the study included a mix of focus group discussions with service users, in-depth interviews with high-level stakeholders and doctors, a ranking instrument used to supplement doctor interviews, and a household survey. These are discussed in turn. 


\subsubsection{Focus group discussions (FGDs)}

The importance of focus group discussions (FGDs) in a study of this type cannot be overstated. They are an economical and effective means of gaining patient perspectives compared with one to one interview. These were the main method for eliciting data on service user perspectives. The information from the FGDs was used to design the household survey. Since there was no previous systematic study on dual practice in Nigeria, the FGDs provided the initial knowledge that aided the survey design. FGDs allow the researcher to understand why the group holds certain views and the group dynamics could offer insights that an individual interview may not. It is a good method to gain more in-depth information to supplement other primary methods, such as the survey method. For example, this approach was used in a widely-cited study by Joseph and colleagues (Joseph et al., 1984) who constructed a questionnaire survey with the help of FGDs. The approach used in the present study to set up the FGDs was to gather together a relatively similar group of participants who had visited both the public and private healthcare facilities in the last 12 months to share their hospital visit experiences. It was an opportunity to explore how service users perceived dual practice, and to determine if, based on their experience, there are benefits and disbenefits associated with service users' recourse to doctors' private practice.

\subsubsection{Sample and sample size}

Four FGDs were carried out in this study. Since this gave twenty-six service users an opportunity to express their views, four FGDs were considered enough to achieve reasonable data saturation and generate appropriate themes for exploration in the survey. It has been argued that, with a qualitative approach, quality of information matters more than number of FGDs conducted. The rule of thumb according to Morgan (1997) is that 3-5 groups will usually be sufficient, as more groups rarely generate further meaningful themes. Against this, some have argued that demonstrating data saturation in real time, in the sense of waiting to determine conclusively that a set of participants generates no further data, is desirable to indicate that no further FGDs need be conducted. Realistically, however, in a multi-method doctoral study such as the present one, pragmatic choices must be made to keep costs and time commitments manageable, and four was judged to be adequate for the study. 
The FGD participants were purposively selected and they included males and females (18 years and above) who had visited both public and private hospitals in the last twelve months. It was decided to use a fairly even mix of genders to achieve a balanced picture across the population of people's experiences in their visits to both public and private health facilities. The number of participants per FGD was between 6-8 persons except in one group that contained 4 participants. Green \& Thorogood (2014) have suggested a group size of 6-12 people but small groups have been shown to be more useful as this gives participants more time to share their experiences in a given topic (Morgan, 1997). In research projects conducted with limited resources, the number of participants may be smaller due to the cost and time-consuming nature of focus groups (Denscombe, 2014). In the present study, these numbers of participants were considered enough to achieve good group dynamics yet avoid problems that arise if too many participants disrupt the flow of the discussion. The FGDs lasted for about 60 minutes each.

\subsubsection{Mode of administration}

The researcher facilitated the FGDs, which were audio-recorded using a digital recorder with participants' permission. A contact person assisted in the recruitment and mobilization of the participants in the study area. The use of a contact person who is already familiar with the community (study area) was helpful not only in mobilizing the participants, but in facilitating the initial familiarization between the researcher and the participants. The consent of the group members was obtained before each session commenced. The four FGDs were conducted between October and November 2015.

\subsubsection{Participants and data collection}

An FGD guide was used to elicit information from the participants. Data were collected on their socio-demographic characteristics, before moving on to the core topics. The questions asked (Appendix 1) included their perceptions of DP of medical professionals; experience visiting public hospitals and private practices of public-sector doctors; self-referral of public patients; benefits and disbenefits of dual practice; and how they would compare the quality of healthcare in public and private healthcare facilities managed by dual practice physicians. In comparing the quality of healthcare in the public sector and private facilities managed by dual practice physicians, the Donabedian healthcare quality model (Donabedian, 2005) was used to 
measure the quality of healthcare services rendered in both sectors. This model consists of three elements - structure (the personnel and the setting where the care is delivered), process (all the activities that take place during the delivery of care), and outcome (indicates whether the goal of the treatment is achieved such as health status, patient satisfaction and cost of care).

\subsubsection{Key informant interviews}

The in-depth interview has been described as a useful method in qualitative research for accessing what cannot be easily observed or accommodated in a formal survey question (Silverman, 2011). Similarly, Low (2013) demonstrates the advantage of interviews over questionnaires. According to these authors, quantitative methods generally follow a hypothetico-deductive logic - they begin with a pre-conceived question which will allow a hypothesis to be tested, and either falsified or left standing. By contrast studies employing qualitative methods usually take an inductive approach by building theory from data. Typically, they construct theories about how people see the world by exploring individuals' subjective perspectives and how they construct meanings from their everyday experiences. This study used interviews with key policy makers and stakeholders who had knowledge about dual practice policy to determine what they thought it meant for doctors and patients, as well as its implications for the healthcare system. The researcher felt that their high-level positions (see below) and experiences of dual practice would mean they were well placed to give relevant information. Informants who had participated in policy making in this area were likely to know the key issues and the arguments about the merits and demerits of dual practice.

\subsubsection{Sample and sample size}

In qualitative research, purposive sampling is used to recruit informants who have specific knowledge about the research question (Low, 2013). In this study, only those stakeholders who have good knowledge of the study aims were recruited and contacted for interview. The relevant stakeholders were sampled using a "Snowball sampling" method. They included the Chairman and Secretary of the Nigeria Medical Association (NMA), the Director of Medical Services in the Enugu State Ministry of Health, the Health Committee Chairman, Enugu State 
House of Assembly, the Permanent Secretary in the Enugu State Ministry of Health, the Chairman and Secretary of the Enugu State Health Board, and the Assistant Director of the Medical and Dental Council of Nigeria (MDCN). A total of 8 interviews were carried out with policy makers/stakeholders. Again, for pragmatic reasons of what was feasible in a doctoral project, it was felt that a small number of high-quality interviews with appropriate informants was more important than a large number of interviews.

The involvement of these high-level stakeholders adds to the study by providing an assessment of the benefits and disbenefits of DP from persons who were involved in policymaking debates about the practice. Given their first-hand knowledge, these informants could provide insights on the nature of the problem and put forward recommendations for solutions.

\subsubsection{Mode of administration}

The researcher conducted the interviews with the selected stakeholders. The respondents were visited in their offices to introduce the aim of the study. They were provided with an information sheet (Appendix 2) and invited to ask questions before signing a consent form. Those who gave consent were asked to agree on the most suitable time and location for the interview. All the interviews were conducted in respondents' offices between July 2015 and April 2016. The interviews were audio-recorded with the participants' permission. This was considered necessary because it was desirable to capture the interview sessions verbatim for subsequent analysis and use of authentic extracts in this thesis.

\subsubsection{Participants and data collection}

An interview guide was used to elicit information from the respondents. The sociodemographic characteristics of the respondents were collected as a prelude to the main questions. These included their highest educational level, designation, and duration in service. The interview guide (Appendix 3) included questions on the current regulation of the dual practice in Nigeria; implementation challenges of DP; benefits and disbenefits of dual practice in the health sector; how doctors are monitored and supervised; perceptions regarding different mechanisms for managing dual practice in the Nigerian context; and regulatory options for dual practice. 


\subsubsection{In-depth interviews with dual physicians and non-dual physicians}

This was the main method used to collect data on doctors' perspectives on dual practice; both dual practitioners and public hospital doctors not engaging in dual practice were included.

\subsubsection{Sample and sample size}

First, a sample of dual physicians in the Enugu Urban was built using a "snowball" sampling method. Only those DPs who work in the public sector and do private practice were selected. A total of fifteen DP respondents were selected for face-to-face interviews between July 2015 and March 2016. In-depth interviews were used to gain an understanding directly from the practitioners about their views on DP. The interviews helped to generate knowledge on how they perceive the problem under investigation, and whether they think differently from other categories of respondents. Second, a group of fifteen non-dual physicians were selected together with the DPs using the "snowball" sampling method. The non-DP interviews took place alongside the DP interviews within the same period. The researcher allowed for a spread in the non-dual physician group to include genders and consultants as well as more junior staff. The study aimed to investigate (if only in a provisional way) whether gender or seniority affected non-engagement in dual practice.

The snowball sampling method is a technique that allows the existing research subjects to suggest others for recruitment from their acquaintances, colleagues and friends. Only one female DP doctor was mentioned by a male doctor during the interview as a potential respondent. This could be attributed to the small number of females involved in DP in Nigeria. All efforts made to contact her to book an appointment failed, and since our objective was mainly to examine the motives for DP only those who were available and consented were interviewed.

Given its nature, the snowball sampling method does not generally produce statistically representative samples. However, it is a practical technique for conducting exploratory qualitative research, especially with a population that is hard to identify or locate. Arguably, in qualitative research the quality of information gathered matters more than interviewing a mix of respondents that precisely mirrors the composition of the larger population of respondents of that kind. Initial moves to identify the DPs in the hospitals and gain their 
agreement to participate failed due to the sensitive nature of DP. Doctors were afraid that the researcher might be reporting back to higher authority on who was engaged in private practice. Snowball sampling was a pragmatic way forward because a personal introduction from the colleague who had passed on the new contact's name to the researcher reassured them that the research did not pose a threat. Despite all this, over ten doctors (both DPs and non-DPs) approached for an interview did not consent, and therefore, were not interviewed.

\subsubsection{Mode of administration}

The researcher approached the selected respondents to acquaint them with the study aims and to seek their participation. They were provided with an information sheet and invited to ask questions before signing a consent form. Those who consented were interviewed at an agreed time and location. Most of the respondents were interviewed in the hospital setting, some in the government hospital, and others in their private facilities. Only a few interviews were done in respondents' homes. Again, the interviews were audio-recorded with respondents' permission.

\subsubsection{Participants and data collection}

An interview guide was used to obtain information from the respondents. Data on their sociodemographic characteristics were collected. The interview guides (Appendix 4a) included questions on perceptions of dual practice; benefits and disbenefits of DP to public patients, and the public sector; conditions that could make them decide to work in the public sector exclusively; motives for dual practice; and if there are other reasons for engagement in dual practice apart from financial gain.

\subsubsection{Ranking of motives for dual practice physicians via ranking questionnaire}

Ranking was an analytical tool used to complement the in-depth interviews conducted with dual practice physicians. This involved including some additional structured questions with a response format that indicated the relative priority that respondents gave to each item within a set of items - in this case, possible motives for engaging in DP (Lavrakas, 2008). The use of the ranking tool was intended to allow the researcher to determine which of a number of commonly-mentioned motives/ or reasons for engaging in DP these respondents rated as most important. 


\subsubsection{Mode of administration}

The researcher administered the structured ranking questions to 15 selected DPs during the indepth interviews. The respondents were asked to identify the main factors that motivated them to engage in dual practice in order of relative importance. A number of authors have identified motives for dual practice such as income enhancement, professional satisfaction and use of private work to enhance reputation in private practice (Hipgrave \& Hort, 2013); clinical autonomy, reputation-building, flexibility of private practice (García-Prado \& González, 2011), professional opportunities, and personal control over practice (Humphrey \& Russell, 2004). The respondents were asked to assign weights (1-10) to the identified factors for engaging in private practice (Appendix 4b). Values less than five denote less importance whereas values above five represent a greater degree of importance. The interview guide (Appendix 5) for non-dual practice doctors was used to explore, their perspectives of private practice, and reasons for not engaging in DP among others.

\subsubsection{Household survey}

This involved the administration of a questionnaire to selected households. Survey design according to (Creswell, 2014) is a quantitative measure of trends, attitudes or perceptions of a population, which is achieved by selecting a sample of the population and studying it in detail, thereby allowing the researcher to use the information generated to generalize the result to the entire population. In this study, a sample of households who had visited both public and private hospitals was recruited and studied to generate information about the extent of self-referral in the public hospitals, the socio-economic status of the service users referred, the cost of referral and so on.

The HH survey was preferred to a patient exit interview for three reasons. A patient exit interview may involve first timers who may not have experienced the influence of DP or faced self-referral in the past, and the hospital setting is not an ideal place to screen for the inclusion criteria. Again, the use of patient exit interviews may introduce bias because the patients may not open up to discuss issues about services from a dual practitioner in the same health facility where they are receiving healthcare services. 


\subsubsection{Sample and sample size}

This study used a cross-sectional multistage sampling design. Multistage sampling connotes a sampling plan with the sampling undertaken in stages (Bryman, 2008). It may involve two or more stages in the sample selection. In multistage sampling also, large clusters are divided into smaller ones in stages to facilitate primary data collection. In the process of selecting the sample, different methods may be applied at different stages. In this study, simple random sampling, purposive sampling and consecutive sampling were applied at different stages.

The software for population surveys in EPI Info 7 was used for sample size calculation. The parameters used for the calculation were the population of Enugu South Local Government Area based on the 2015 population projection, which was 259,000 (NPC, 2006), power of $80 \%$, confidence limit of $95 \%$, and expected frequency of $50 \%$. We expected a $95 \%$ response rate since the questionnaire administration was researcher-administered. So, allowance was made for non-response by increasing the sample size to 404. This was achieved by dividing our initial sample size of 384 by 0.95 , which gave a target of 404 . However, in the event a total of 407 questionnaires were administered and completed, following a pre-test. The pretest was conducted with a $5 \%$ of the intended sample (20) respondents who were not then included in the final questionnaire study.

\subsubsection{Sampling procedure and data collection}

Multistage sampling was used to select the sample. There are three local government areas (LGAs) that make up Enugu Urban. These are Enugu North, Enugu South and Enugu East. Enugu South LGA was selected using a simple random pick. Enugu South has a population of 198,032 and population projection of 259,000 in 2015 (NPC 2006). This LGA has five residential areas that are mostly urban: Achara-Layout, Uwani, Maryland, Gariki and IdawRiver. Each of the five residential areas has slightly different growth indicators in terms of demography (population density), economy, commerce and infrastructure (Iyi, 2014). There are also variations in access to good roads in each of the areas. Some of the residential areas have better access roads than others. Also, some houses in some of the areas have high walls and gates. 
Therefore, two of the five residential areas were selected purposively for questionnaire administration. Purposive sampling was used to avoid the selection of residential areas with accessibility challenges. The inclusion criteria used were (1) accessibility to potential household respondents in terms of absence of bad roads and high wall fences /gates, and (2) a good house numbering system. Similarly, within the two selected residential areas, inclusion criteria were used to select the streets for questionnaire administration. In this study, lanes, closes, short streets and streets with markets were excluded. The streets that met the inclusion criteria were listed, and simple random sampling was applied to select four streets in each of the two areas. Then after that, the systematic random sampling was used to select houses for questionnaire administration using even or odd numbers. In addition, where a house visited was home to more than one household, consecutive sampling was used to administer the questionnaire to other eligible households occupying the building. Having selected households, the researcher recruited survey respondents based on their previous hospital admission experience. The inclusion and exclusion criteria that guided the administration of the questionnaire to the respondents was that only respondents who had first visited a public hospital and then gone to a private hospital or clinic in the last 12 months were administered the questionnaire. The researcher excluded from the study those who enrolled in the National Health Insurance Scheme (NHIS). This group of healthcare consumers have the choice of choosing only one provider - public or private - for their hospital visits. Also, self-referral rarely affects insurance policy holders. However, a substantial number of households, over 90 per cent are not covered. The author acknowledges that because of the sampling strategy of selecting districts and then streets with Enugu Urban area, there may be some clustering of respondents with particular social or demographic characteristics. Clustering may mean that the study sample differs from a simple random sample, and an adjustment made to counter this is discussed in section 2.5.4.

\section{Mode of administration}

The questionnaire was researcher-administered. This approach was used to avoid ambiguity and variations in the understanding and responding to the questions. The anticipated variations in understanding the questions stemmed from the low educational level of some of the respondents, which increased the potential for misunderstanding of questions. The 
respondents waited as the researcher asked the questions and ticked their preferred responses. Those who stated that they were too busy to give sufficient time to complete the questionnaire were excluded. No questionnaire was left uncompleted or for later collection. However, a few questions that respondents were unable to answer were left blank. Data for the HH survey were collected over 90 days from late January - April 2016.

\subsubsection{Questions}

Data on the socio-demographic characteristics of respondents were obtained as well as information on hospital visit experience, the extent of self-referral, estimates of costs incurred by referral from the public to the private sector, views on the benefits and disbenefits of dual practice to service users, general perceptions of dual practice, and opinions of the relative quality of public healthcare and private healthcare managed by dual practitioners. Regarding this last area, the questionnaire incorporated elements from the Donabedian healthcare quality model (Donabedian, 2005) to measure the level of quality of healthcare services rendered by DPs in both sectors. This model is simple and is acknowledged as a method for healthcare quality investigation (Heather \& Richard, 2011; Robert, Elizabeth, McGlynn, \& Paul, 2000). It considers quality in terms of structure, process and outcome. The structure aspect consists of the personnel and the setting where the care is delivered, while process deals with all the activities that take place during the delivery of care. Outcome measures indicate whether the goal of the treatment is achieved, for instance, improvement in health status, patient's satisfaction and cost of care (Hanae, Mohamed, \& Noureddine, 2013). The questions were structured to reflect these three aspects of healthcare quality using five-point Likert scales (see Appendix 6).

\subsection{Qualitative data analysis}

\subsubsection{Analysing FGDs}

The FGDs were audio-recorded and transcribed verbatim. Data were organized using NVivo Version 10. The transcripts were read several times to improve understanding of the concepts and meanings in the text. The researcher considered familiarisation with the data important as the analysis involved repeated reading of the transcripts with the intent of searching for meanings, patterns, and processes (Saldana, 2013). The analysis involved a combination of 
deductive and inductive coding. The deductive coding involved the application of themes from the known literature that appeared relevant (and were reflected in the research questions), while the inductive coding involved adding some new issues and ideas emerging from the data.

The initial coding involved a first attempt to reveal the meanings and patterns emerging from the data. This helped to generate a provisional list of ideas within the data and compared their importance with other data. A process of re-coding was applied as themes and sub-themes continued to emerge and change. This helped the researcher manage and filter the data to focus on emergent patterns, refine themes and work out their linkage to relevant concepts. Finally, the major and minor themes were reviewed for relevance, and in the process some themes were merged, and others discarded.

Initial coding generated 169 codes from the main data set. The next phase was sorting the codes into potential themes. Using NVivo, Version 10, thematic nodes were created where the relevant codes were sorted into different potential themes. At this point, sub-themes were also created from the main themes to enrich the analysis.

The final phase of analysis was devoted to reviewing the themes. Some of the themes lacked enough data to support them and were discarded, while others were merged into an overarching theme. Also the internal 'homogeneity' and external 'heterogeneity' of data were considered (Braun \& Clarke, 2006). Data within a theme that did not seem to cohere together meaningfully were dropped or separated, while other themes that did not have clear distinctions between them were merged together. In some areas analysis was advanced by using a visual representation of the themes and subthemes (Appendix 7). 


\subsubsection{Visualising the coding process}

The process is illustrated in figures 1, 2 and 3 that follow.

Figure 1: Summary of 'stages' of data analysis for the FGDs and IDIs

Stage 1: Early transcription of each FGD after the session and storing them in a folder designated for the study

Stage 2: Proof reading of the FGD transcripts and initial familiarization with data

Stage 3: Reading and re-reading each of the FGDs several times until I was 'immersed' in the data

Stage 4: Initial open coding of the FGDs using Microsoft Word

Stage 5: FGD transcripts imported into NVivo version 10 with some changes to the coding done in MS Word

Stage 6: Thematic nodes were created followed by sorting the different codes into relevant themes and subthemes

Stage 7: Continuous review of themes and subthemes by going back and forth until finalised 


\section{Figure 2: Showing initial coding in MS Word}

\section{Q1. What is your perception of dual practice of medical practitioners in Nigeria?}

P5: I attend both government and private hospital. Government hospital has all necessary equipment and facilities for healthcare services. If you deliver a premature baby, for instance, they have an incubator to make the baby strong. In the private hospitals, they may provide all the necessary things for your treatment, and thereafter, you may be charged by instalments if you are not able to make a one-off payment. But in the government hospital, they would simply prescribe all the drugs and ask you to buy them at a private pharmacy outside the hospital. If you do not have the money to make the purchases, they will not attend to you. For example, like what happened to my sister who went to a government hospital during her pregnancy for her baby who was said to be lying in the breech position. After she was prescribed some drugs and all the things she had to buy, the husband went out to look for money to purchase the drugs, and while he was running about looking for the money the baby died in the womb. A private hospital would have given her all the needed attention and performed the operation and then allow her to pay gradually until she finished the payments.

P2: I think they feel that what they are being paid by the

Dps give less government is not enough and they need to have their own private practice to augment. So, they gain extra income from both sides - government and their private practice. Dps augment salary

attention in public

Dps give less attention in pub

Dps spend more time in private

Pub hosp is equipped

Good care in private

Instalment payment in private

Out of stock of drug in pub

Deposit before treatment in pub DPs augment salary

Dps augment salary


Figure 3: Showing initial codes imported into Excel

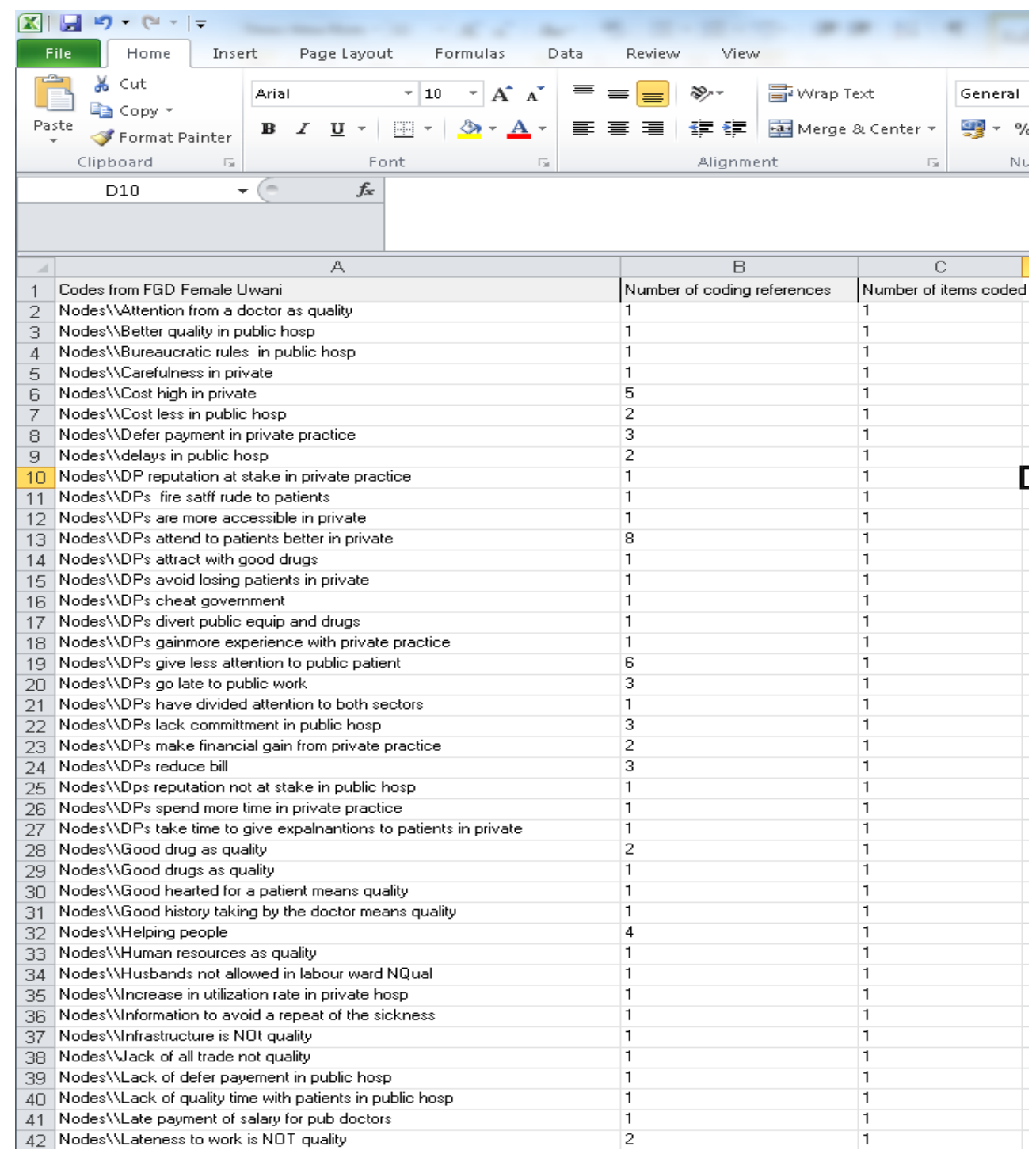

\subsubsection{Key informant interviews}

Data analysis adopted a similar approach to that used with the FGDs. Unlike the focus group, the initial coding generated over 700 codes from the main data set. For example, of the visual representation of a theme and subthemes (see Appendix 8). Ten main themes emerged from the key informant interviews. 


\subsubsection{Interviews with dual physicians and non-dual physicians}

Data analysis adopted a similar approach to that used with FGDs. However, the initial coding of this report yielded 726 codes. A visual representation of one of the themes and other subthemes are found in the appendix (Appendix 9). Overall, six main themes emerged from the DP and non-DP interviews.

\subsection{Quantitative data analysis}

\subsubsection{Ranking of motives for dual practice physicians}

This exercise had been added to the doctor interviews to measure the relative importance of different motives for engaging in dual practice. The data on the strength of different possible motives derived from ranking scale questions were analysed using SPSS Version 22. The "Explore" procedure under descriptive statistics in SPSS was used to obtain the mean scores and standard deviation, range and interquartile range (Table 19). The variable with the highest mean score was ranked as the most preferred reason for engaging in dual practice.

\subsubsection{Household survey}

This section covers the quantitative data analysis of the household survey. Data analysis was carried out using SPSS Version 22, STATA Version 10 and STAT transfer software. Frequency tables and percentages were generated to represent the socio-demographic characteristics of respondents. The Socio-economic status index was disaggregated into quartiles (4 groups) with Q1 (most poor) and Q4 (least poor). The process involved the use of STAT transfer to transfer data from SPSS to STATA. In STATA, the variables of interest the household asset holding, living conditions and household weekly food consumption obtained from the household questionnaire were used to create the socio-economic status group of our survey sample. The SES index created was then transferred back to the SPSS for further analysis.

Principal components analysis (PCA) in STATA Software was used to generate the socioeconomic status (SES) index of respondents (Filmer \& Pritchett, 2001). The initial aim was to use the socio-economic status index to examine whether there were systematic differences in the impacts of dual practice across the different SES groups. However, this was not pursued 
as the referral group $(n=34)$ was considered too small to carry out a sub-group analysis. Instead, the SES index helped to examine the SES group that is mostly self-referred by dual practitioners. The variables that were included in the SES index were ownership of key assets such as car, motorcycle, radio, refrigerator, television set, bicycle, and grinding machine together with household weekly food expenditure. In Nigeria, about $62.8 \%$ of wage earners spend between $70 \%$ and $80 \%$ of their earnings on food (Federal Office of Statistics, 2001). Therefore, the cost of food is used as a proxy for income since it is not easy to collect reliable information on household income in Nigeria. This method of determining the SES has been validated and used in previous studies in Nigeria (Eze, Ochonma, Ajuba, \& Obikeze, 2015; Okoronkwo, Onwujekwe, \& Ani, 2014; Onwujekwe, Hanson, \& Uzochukwu, 2012; Vyas \& Kumaranayake, 2006).

\subsubsection{Descriptive Likert analysis}

A Likert scale is a five or seven-point scale used to allow individuals to indicate how much they agree or disagree with a given statement. Such rating scales are mostly used to measure attitudes or opinion by asking respondents to respond to statements on a given topic, using a fixed-choice response format. By asking people to indicate the extent to which they agree or disagree to a statement, a question taps into the cognitive and affective/emotional aspects of attitude. In this study, Likert-type scales were used to understand respondents' views about benefits and disbenefits of DP to service users. They were also used to explore the perceptions of respondents regarding the motives for dual practice of public-sector doctors and to compare the quality of healthcare of public and private practices of the same doctor using the Donabedian three-part model of healthcare quality. Participants 18 years and above who had visited both public and private hospitals were administered the questionnaire, composed mainly of Likert-type items. The scale comprised: 1= "Strongly disagree", 2=" Disagree", 3=" Don't know", 4= "Agree", and 5= "Strongly agree". A simple descriptive analysis of the Likert means (and 95\% CI) was used to measure strength of agreement or disagreement. 


\subsubsection{Adjustment for clustering}

One difficulty with the sampling strategy adopted in this study is that the fact that given areas were selected and then streets were selected within areas opens the possibility that there were clustering effects. For example, the characteristics of the sample of households selected from a given street may be different from a sample of households selected randomly from streets across the whole area, because persons living in a given street may share sociodemographic characteristics different from those of other streets. This means that statistical tests and processes involving the assumption of a "simple random sample" are invalid and may end up giving biased results. The analysis was adjusted to allow for this possible clustering effect.

Two adjustments were undertaken, firstly for the two larger areas and then for the streets. The cluster analysis was carried out to account for clustering in the sample design using the "svy" command in Stata. This command allows the user to declare complex sample design features of a survey to Stata so that the standard error and confidence interval can be adjusted (Stata Manual, 2013).

Since we have adjusted for clustering we have now obtained the true significance test for age, sex and SES of those referred from the public hospitals (See Tables 14 and 16). Stata automatically calculates the F statistic rather than the expected chi-square, to account for the clustering (Rao and Scott, 1981).

\subsubsection{Sensitivity analysis of value of hours lost}

Sensitivity analysis in Excel 2010 was used to calculate the doctor's hourly pay in the public sector (Appendix 10) for a normal routine hour. The hourly pay helped the researcher to estimate the magnitude of the value of hours lost to dual practice due to absences. First, different hypothetical scenarios were created to estimate hours lost per week. These scenarios are 5, 8, 10, 12, 15 and 20 hours per week with 5, 8 and 10 hours as the lower band, and 12, 15 and 20 hours as the upper band. The aim of creating different hypothetical scenarios is to establish the extent of the value of hours lost to the public system of DP under different situations. In this instance, the intention was to show the level of the value of hours lost represented by government salaries paid for work that is never done. These values were estimated under different scenarios. These scenarios were not built on any empirical data. 
The parameters used for the calculation were the official 40 hours working week (normal routine hours), and monthly gross pay of doctors on different grade levels (1-7). The researcher obtained the average monthly salary of doctors on different grade levels by summing up the salaries in each grade level and divided by the number of incremental steps (the steps to pass through before moving to the next grade level) to obtain the average monthly salary of doctors within each grade level. It is important to note that the salary was based on monthly gross pay and not net pay. It was decided not to attempt to get data on actual salaries of employed individuals at the different grades because this would have been sensitive, and the analysis might have allowed individuals to be identified. The averaging of salaries across the increments per each grade and the use of gross salaries mean that it is not possible to make inferences about individual's pay. The Excel Worksheet example used for creating the different hypothetical scenarios is found in the appendices (Appendix 11).

Next the supposed hourly pay was calculated. The first step was to multiply the basic 40 hour working week by 45 weeks (with seven weeks removed as the standard holiday entitlement) to obtain 1800 hours paid for per year, and then dividing this by 12 months to obtain 150 hours per month. To obtain the hourly pay, the average monthly salary was divided by 150 monthly hours. This was applied to all the grade levels to obtain the hourly pay of doctors at different grade levels (See Appendices 12-17) for the outputs from the sensitivity analysis of normal routine hour loss.

At the same time hourly pay for on-call hours worked over the standard 40 hours was also calculated (hours that might also be lost to DP). A similar approach to that described above was applied. There was no standardised number of hours to spend on on-call hour as there was for the main 40 hours of normal routine hour. Different departments in the hospital have varied on-call hours depending on their workforce strength. The on-call hourly pay was obtained by first obtaining the average monthly on-call hours allowance per grade level. Then the average monthly on-call hours allowance was divided by 150 monthly hours (as above) to obtain hourly pay for on-call hours (Appendix 18). In this case, the lower band represents 5, 6 and 7 hours lost in respect of paid on-call hours when doctors absent themselves for DP, while 8,9 and 10 hours lost denote the upper band for the outputs from the computation of 
on-call hour loss (Appendices 19-24). This may lead to an uneven estimate of the value of hours lost to DP across departments. As described above, these scenarios are hypothetical.

\subsection{Rigour and validity}

The FGD sessions were conducted in the respondents' language of choice. However, those who were fluent in English were encouraged to use that language, as that would avoid any problem of distorting meaning through translation. Interviews were audio-recorded and were transcribed for near-immediate review by the researcher to ensure accuracy. Immediate review of each interview helped the researcher keep track of similarities and differences in the information generated in successive interviews, and sometimes suggested issues that could be probed in future. Also, this helped to avoid loss of vital information, which could occur if the entire analysis was left until the end of field work. A small number of pilot exercises were done in advance before the field work. The data obtained were not analysed as it was not a standardized instrument. Instead, these exercises helped the researcher to refine some questions or conceptual terms that had not been well understood by respondents. Some questions at this stage were either expunged or amended to ensure validity and reliability. The researcher tried to attend to the "craft" of fieldwork to maintain rigour and ensure data reliability, by such means as seeking corroboration by multiple respondents and note taking when significant events occurred. Triangulation of findings from the interviews, FGDs and the survey, in the context of the study objectives, enhanced analytical rigour. This triangulation process made it easier to identify consistencies and inconsistencies arising from the responses across respondent categories.

\subsection{Ethical considerations}

Ethical approval for the study was obtained from the University of Nigeria Teaching Hospital Committee on Medical and Scientific Research Ethics (Appendix 25), the Enugu State Ministry of Health (Appendix 26), and the Research Ethics Committee of the College of Human and Health Sciences, Swansea University, UK (Appendix 27). Interview and survey participants were required to indicate consent on a standard consent form (Appendix 28). 
Where a participant was not literate enough to sign, it had been planned to substitute a thumb print, but in the event such a case did not arise. In the case of FGDs, verbal permission to go ahead with the study was sought and was audio-recorded as evidence of consent. All participants were informed of their right to withdraw from the study without penalty. Data were stored in a locked file cabinet in an office where there is a minimal access to third parties. The principle of informed consent was strictly adhered to, and the identities of participants in interviews and focus groups will not be revealed in printed outputs from this study. The posts of senior stakeholders are sometimes indicated, but in a few instances have been disguised by use of a generic term. With the FGDs there was no guarantee for confidentiality because all the participants were able to witness what was said during their session.

\subsection{Literature review methodology}

The literature review used a non-systematic "narrative" review approach, though one that followed a clear logic. The aim of this review was to map out what is known about dual practice from previous studies and perhaps extrapolate from relevant past findings to the present study. The approach has similarities to a systematic review in that it started with an online search of selected bibliographic data bases, but it was extended on an ad hoc basis to include additional sources identified by other means.

\subsubsection{Search strategy}

The review searched for literature in Medline, Web of Science, Pub Med, and CINAHL. Other sources used to identify literature included Google Scholar, Google search engines, and Mendeley. Grey literature was also accessed using the World Health Organization Library Database (WHOLIS). In addition, reference lists of key papers were snowballed to track other papers found relevant to this study. A similar process was followed using the Google Scholar citations facility to identify papers that had referred to key items. There was also a physical search done in the library. No rigid time period was set for the searches. This is because the study is not time bound, and restriction of the search to a time frame would not be of any relevance in the study. Both indexed and free-text terms were used (see below) to extract relevant articles for the study. The literature search was carried out in the English language. 


\subsubsection{Inclusion and exclusion}

All the titles and abstracts obtained from the search were filtered to ensure that they are appropriate to the objectives of the study. Therefore, articles that are relevant to the overall argument of dual practice of medical professionals and regulatory mechanisms associated with the practice in both developed and developing countries were included, while those that are tangential to the study objectives were excluded.

The key search terms used included \#S1 -(multiple job holding and dual practice and moonlighting ) AND economic impact analysis, \#S2(multiple job holding and dual practice and moonlighting) AND impact analysis \#S3 (doctor* OR physician* OR family practitioner* OR general practitioner*OR consultant*OR GP*) AND \#S4 (dual practice* OR dual job holding OR dual practitioner*) \#S5 physician* OR doctor* OR medical personnel OR medical professional* OR medical worker* OR medical provider OR medical staff, (dual or double OR multiple OR several OR secondary OR additional OR supplement*) N4 employment. \#S5 "Moonlighting" \#122. The combination of Boolean phrases yielded different number of relevant articles, while in some there was none. The combinations of different key words used for the search were repeated in the different data bases. It may be important to note that dual practice of healthcare professionals is not yet a well-researched area, and therefore, has only a limited academic literature. The references identified through some key articles' reference lists were retrieved using Web of Science, Mendeley Reference Manager, Google Scholar and Google search engines.

\subsection{Data management}

The electronic copies of the identified published papers were retrieved and uploaded to Endnote being the main reference software used. A folder was also created on the system where all the downloaded articles were sorted by theme and stored. The papers were also uploaded to other external hard drives for back up.

\subsection{Assessing quality of study articles}

The initial criteria used to assess the quality of the study materials were adapted from Aveyard (Aveyard, 2014). The papers were read and, in some cases, re-read to understand the 
objectives, methods, results and conclusion. Thereafter, a table (Appendix 29) was created and used to summarize the information from each of the reviewed papers. The review helped to identify the strengths and weaknesses of the papers. At this point, papers that were not relevant to the objectives of the study were excluded. Finally, themes were generated from the findings, which form the domains of the literature review. 


\section{Literature review}

\subsection{Introduction}

In this chapter, we critically review empirical and non-empirical studies, grey literature, and other published relevant articles on dual practice (DP) among medical professionals. Our review specifically centres on five areas of concern in this study. These are the benefits and disbenefits of dual practice, self-referral by dual practice physicians, motives for dual practice in the Nigerian health sector, a comparison of the difference in quality between services offered in the public sector where a dual practitioner works and those provided in their private practice, and an examination of different regulatory mechanisms for managing dual practice in different healthcare systems. Each of these areas is discussed under separate sections.

\subsection{Benefits and disbenefits of dual practice among medical professionals in the health sector}

In this section, we review the literature on the benefits and disbenefits of dual practice. The proponents of dual practice argue that multiple job-holding has positive effects on the public system. On the other hand, the opponents view the potential negative implications of the practice on the health system.

First, we look at what might be considered the advantages of physicians' dual practice. In this regard, we base our review on DP and access to health care, DP and health care quality, DP and skills development, and compensation for giving up full-time private practice.

\subsubsection{Dual practice and access to health care}

Access to health care features as a major item on the policy agenda of most healthcare systems. It creates equity between the poor and the rich and helps to close the gap of inequality in the health system. The proponents of DP have argued that governments of low and middle-income countries (LMICs) lack sufficient incentives to attract public sector doctors to provide services in rural areas due to poor funding. However, with DP the government would be able to recruit and retain public doctors if there is an incentive allowing 
them to engage in dual practice to raise extra private income. They argue that if DP is permitted then access to healthcare is expanded. For instance, when wealthy patients avoid long wait-times in the public sector by opting for private treatments, this frees space in publicsector facilities so that access is expanded for both the poor and the rich, as well as for both rural and urban dwellers (Berman \& Cuizon, 2004; García-Prado \& González, 2011). In principle, this sounds reasonable. For instance, in South Africa private practitioners are offered contracts to service rural areas (Jan et al., 2005). In Bangladesh, Gruen et al. (2002) found that DPs spent 15 hours per week in private practice, and that this expanded services to rural communities. However, given the fact that the hourly wage from private practice is higher in the urban than rural areas, few doctors may respond to such incentives by moving to rural areas. For example, a study on physician shortages in rural Vietnam shows that only $20 \%$ of private practitioners opted for employment in rural areas (Vujicic, Shengelia, Alfano, $\&$ Thu, 2011). This may suggest that DP doctors' motivations may be economic, rather than the expansion of health care access. In Nigeria, also, there is a higher concentration of private practice in cities, where richer and middle-income earners live than in rural areas.

From the above argument, it might be contended that DP allows governments to recruit and retain qualified doctors at a low cost due to the incentive for private income. However, evidence has shown that absenteeism, late reporting to work, and poor commitment to government work are common among DP physicians (Abera, et al., 2017; Chaudhury, Hammer, Kremer, Muralidharan, \& Rogers, 2006; Ferrinho, Lerberghe, Fronteira, Hipolito, \& Biscaia, 2004b; Gruen, et al., 2002), which inevitably reduces the quantity of services offered. Evidence from developed countries is also conflicting about whether DP expands or restricts access to health care services. For example, a study that analysed consultants' NHS and private incomes in England found that full-time NHS doctors engaged in private practices invested more time in their private work by exceeding the $10 \%$ income threshold from private remuneration (Morris et al., 2008). This evidence supports Yates (2000), who found that some NHS consultants devoted an excessive amount of time to private patients.

Conversely, (Bloor, Maynard, \& Freemantle, 2004; Eggleston \& Bir, 2006) found that consultant surgeons in the English NHS on part-time contracts with the freedom to engage in dual practice undertook more recorded NHS activity than the full-time doctors. Similar 
evidence has been reported in Denmark (Sochas \& Bech, 2012) and in Norway (Johannessen \& Hagen, 2014). These authors' views seem to show that DPs are still productive as their non-DP counterparts.

Despite the above argument, significant earnings from private practice have been reported in LMICs. In Thailand, earnings from private practice among public doctors constituted $55 \%$ of total income (Hanvoravongchai, Letiendumrong, Teerawattananon, \& Tangcharoensathien, 2000), while in Nigeria $68 \%$ of public doctors make more money from supplementary work than from salaries (Akwataghibe, et al., 2013). This evidence may suggest a strong incentive to spend more time in the private sector. This has the potential to shrink public sector services.

\subsubsection{Dual practice and healthcare quality}

Healthcare quality has different meanings and, depending on the area of interest, it can be defined in various ways. In this study, we apply the Donabedian approach to healthcare quality (Donabedian, 1980). Thus, quality is defined in three dimensions: 1) the structure element of quality - the physical attributes of the settings where care takes place; 2) the process element of quality - meaning what is done in the process of providing care; and 3) the outcome element of quality - in terms of the effects of care on patients' health status. For a detailed definition and analysis of health care quality (see subsection 3.16).

The proponents of DP argue that it improves healthcare quality because the government allows dual practice as an incentive to employ and retain experienced doctors in the public sector. The general perception among the proponents is that such an incentive would motivate dual physicians to improve the quality of their care in the public sector, and boost their reputations to attract private patients (Berman \& Cuizon, 2004; Biglaiser \& Ma, 2007; Eggleston \& Bir, 2006; García-Prado \& González, 2011). There may be a conflict of interest; rather than increasing efforts to improve quality in the public sector, dual physicians may lower the quality of their care as a way of attracting patients to their private practice. The evidence in Peru shows that private practice widens the quality gap between public and private sectors (LaGrone et al., 2017). The authors found that DP physicians prioritised the better-paid sector by choosing shorter surgical procedures and ignoring the ones that may lead 
to better outcomes, but which are longer and more time-consuming, just to avoid being late to the private clinic due to a large pay differential. Similar evidence was found in Iran and Palestine (Alaref et al., 2017; Moghri, Arab, Rashidian, \& Sari, 2016), and Kiwanuka et al. (2010) showed that quality of care is compromised in the public sector.

On the other hand, poor quality care may be a response to public system work conditions, such as poor facilities, poor salary, a lack of equipment and delays in paying salaries. These conditions are typical of the LMIC situation, which results from sub-optimal government strategies for health care governance and provision (Hipgrave et al., 2013). In Palestine, it has been shown that the inability to separate the multifaceted factors associated with poor quality of care from the actual effect of dual practice blurs the boundary between the conditions in the healthcare system on the one hand, and doctors' influence on quality on the other hand (Alaref, et al., 2017). Similarly, Socha \& Bech (2011) stated that poor management and organisational culture affect physicians' motivations and performance, which makes it difficult to separate governance and management issues, and the quality of care expected of physicians. Inasmuch as the hospital sub-system may lack good organisational culture, doctors' individual characteristics — such as whether they are profit maximisers, or missionoriented public servants - may influence quality of care.

\subsubsection{Dual practice and skills development}

Dual practice has been argued to offer practitioners the opportunity for clinical skills development. Since the health market is dualised with private and public markets existing side by side, patients of different socio-economic groups are free to attend both sectors. Disease types that are treated in both sectors may differ. Doctors who straddle both sectors are believed to gain additional skills, as doctors' experience may depend on the types and number of cases handled.

Some previous studies have found that DP might enhance the development of clinical skills among surgical residents (Berman \& Cuizon, 2004; Jamshidi \& Reilly, 2008; LaGrone, et al., 2017). It has also been reported that moonlighting helped resident doctors in Emergency Medicine to gain experience outside their primary job (Chisholm, 2000). Medical practice differs across health systems; for instance, in the UK there is a comprehensive universal 
coverage, although a small percentage of people with a top-up private health insurance exist. The public sector is more developed and may have more and better facilities than the private sector. Despite the existence of a comprehensive universal coverage, the NHS DPs still value professional opportunities to practice new skills in the private sector - cases that may not come to the public sector can be handled in the private sector (Humphrey \& Russell, 2004).

In India, as in several other countries, medical tourism (MT) has transformed health services into a commercial trade meant to raise revenue. The public sector in India is described as very poor and with poor medical facilities; without subsidies about $70 \%$ of the Indian population may not be able to afford a service (Qadeer \& Reddy, 2013). The private sector in India offers that opportunity for skills development with a high volume of international patients and stateof-the-earth equipment. Nigeria has a similar commercialised private market, but this is not developed like that of India. There is a high volume of activities in the private sector compared with the public sector, with $68 \%$ of public doctors making more money from supplementary work than from their salary (Akwataghibe, et al., 2013). There is a large patient clientele in the private sector in Nigeria, though with no international patients, which shows that a lot more cases are handled there than the public sector. The DP physicians, therefore, acquire skills due to clinical case diversity. A study of how doctors choose where to work shows that career and professional development prospects ranks second after a good workplace (Heikkilä et al., 2014). Physicians who work exclusively in the public sector may have limited skills and experience in some areas of medicine and there could be a limit to what they can do. Of course, the same may be said of many physicians practicing exclusively in private facilities, where the medical equipment available is less sophisticated than in a larger government hospital and access to specialist colleagues may be more limited.

The important question, therefore, is how the skills acquired from the extra private work enhance dual physicians' performance in the public sector. It may have a huge benefit if both sectors complement each other. The skills acquired from the public sector may help the private work and vice versa, but where more attention is paid to private practice, as it is in most health systems in LMICs, the skills may only help to advance dual physicians' private work. 


\subsubsection{Compensation for giving up full-time private practice}

Many governments permit dual practice as compensation for giving up full-time private practice or offer financial incentives for those who give up DP for a full-time work in the public sector. There seems to be a liberal approach to dual practice in several healthcare systems (Hipgrave \& Hort, 2013), and countries such as the United Kingdom (Humphrey \& Russell, 2004), Norway (Johanessen \& Hagen, 2012), Denmark (Socha \& Bech 2011), Bangladesh (Gruen et al., 2002), Egypt (Zhang, 2015), Nigeria (MDCN, 2008) and Uganda (Ligia, 2014) permit dual practice among doctors in one way or another. Taking things one step further, some countries offer additional compensation to doctors who willingly forgo full-time private work for exclusive public work. Different types of financial incentives may be offered. For instance, the governments of Portugal, Thailand, Spain and Italy provide exclusive contracts to doctors as an incentive to remain in the public sector (Jan et al., 2005; Gonzalez et al., 2007; Berman \& Cuizon, 2004). In sub-Saharan African countries, governments generally have a permissive attitude towards DP as a way of mobilising resources and retaining qualified doctors (Eggleston \& Bir, 2006). In other instances, governments may allow doctors to undertake extra private work to supplement incomes. For instance, the Code of Medical Ethics in Nigeria allows dual practice after official work and when not on-call (MDCN, 2008).

Although it is not unusual for governments to use incentives to attract physicians to the public sector, the capacity to monitor and check the activities of physicians to ensure that a "good idea does not turn to a bad one" seems paramount. For instance, in the 1990s the government of Croatia permitted private practice as a reward to specialists in the public sector (Gabric \& Lazic, 2013), but following the abuse of the privilege, the practice was banned. A similar sequence of events took place in Greece in 1995, when the Greek government increased doctors' salaries by 250 per cent to get them to cease private practice due to illegal practice observed among senior doctors. The policy failed as many senior doctors resigned from public service due to the large profit available from the private sector (Mossialos et al., 2005). The above instances may suggest that incentives can be perverted and, unless the authorities are able to control the professionals, a public system's service delivery may be affected 
negatively, even with the supposedly 'best' incentives. Further research is needed to explore this theory in detail as well as its potential effects on the public sector.

\subsection{Disbenefits of dual practice}

In this section the arguments of the opponents of dual practice are critically examined. The following areas of the debate are discussed.

\subsubsection{Waiting lists}

The current debate on dual practice has identified long waiting lists in public facilities as something that dual practitioners arrange by design (Berman \& Cuizon, 2004; Gabric \& Lazic, 2013; Socha \& Bech, 2011). The argument holds that dual physicians may purposely create long waiting lists in the public sector to generate demand for their private services (Brekke \& Sørgard, 2007; Jan, et al., 2005). In contrast, Sochas \& Bech (2011) argue that if the dual physician is on a fixed salary, he may not have any incentive to treat a higher number of patients; thus, long waiting times are not to their advantage. On the other hand, long waiting lists can boost demand for private care, which inevitably benefits dual physicians.

Developing country experience shows that absenteeism, truancy, and late reporting for, and early departure from work are various ways of building up long waiting lists (Abera et al, 2017; Gruen et al., 2002; Osuagu, 2010), indirectly creating demand for private practice. For instance, a consultant may report to work at $11 \mathrm{am}$ or $12 \mathrm{pm}$ and leave at $1 \mathrm{pm}$, and those patients who could not see him or her might be asked to return another time. The alternative for any patient who cannot wait long in a public facility is to attend the dual physician's private clinic. However, patients from different socio-economic groups may not all be able to endure the long wait. The least poor can easily drop from the waiting list to seek private care. Nigeria's public sector situation shows that patient waiting times in public health care centres pose a challenge (Olowookere, et al., 2011; Umar, et al., 2011). Those who can afford private care may be moved by relatives or advised by health workers to visit dual physicians at their private clinics.

Yates (1987), one of the foremost contributors to the private practice debate, argues that waiting lists are caused by the selfishness of consultants. He further argues that there are 
indeed abuses of the NHS through private medicine, but that these remain largely unproven due to a lack of any mechanism to study the surgeons' activities (Yates, 1987). Although private practice can reduce the waiting time/queue in the public sector as an alternative, it is not without a higher cost to patients. The argument about private practice tends to take two directions; in defence of dual physicians or of the public system. Most doctors argue that the public system is inefficient and underfunded and that seems a fairly safe defence for their decision to undertake private practice according to Yates (1987). Conversely, previous studies seem to suggest that dual physicians are as productive as their NHS full-time counterparts (Socha \& Bech, 2011; Johanessen \& Hagen, 2014). However, the analysis that looked at consultants' NHS and private incomes in England showed a positive association between mean private income and length of NHS waiting lists across specialties (Morris et al, 2008). This suggests that waiting lists in the public sector, if not checked, could create demand for private practice. Further studies are needed across health systems to establish the association between private practice and long waiting times in the public system.

\subsubsection{Competition for time/shifting of attention to private practice}

Public-on-private work has been criticized for restricting access to health care services in the public sector (Berman \& Cuizon, 2004; García-Prado \& González, 2011). Previous studies (Chaudhury, et al., 2006; Ferrinho, et al., 2004b; Macq, et al., 2001) have shown that competition for time between public and private work limits access to health care. For example, in Venezuela, and Costa Rica health workers missed their contract service hours by between 37\% and 30\%, and 65\% respectively (Ferrinho et al., 2004b). In India, Bangladesh, Indonesia, Peru and Uganda 35\%, 40\%, 25\% and 37\% of absenteeism was recorded, respectively, among health workers (Chaudhury, et al., 2006; Ligia, 2014), and in Nigeria, $46.9 \%$ of absenteeism was recorded with doctors (Isah, et.al., 2008). Thus, attention and efforts may be concentrated on private work, where there is a higher profit margin. This is supported by Macq et al. (2001), who showed that competition for time may result in the transfer of resources out of the public sector through reduced availability, which accounts for a loss of value equivalent to $27 \%$ of salary among public health managers in developing countries. Absenteeism by health workers may restrict a patient's subsequent visit to a public facility and could lead to seeking an alternative health care provider in the private sector. But 
the question of whether these absences are associated with private practice may need further research to establish.

A recent study in three African cities (McPake, et al., 2014) shows that the choice of allocation of time in the private sector by dual practitioners depends on a number of factors, such as number of dependants, whether holding a specialisation and whether having enough private patients. The allocated time for private practice in Nigeria starts from $4.00 \mathrm{pm}$, after the official public-sector work time from $8.00 \mathrm{am}$ to $4.00 \mathrm{pm}$. The dual practitioner in principle can only resume private work after $4.00 \mathrm{pm}$, but whether this is strictly obeyed is debatable. Some dual physicians may be busier than others, particularly those who specialise in areas not practiced in the public sector. For instance, in Nigeria, in vitro fertilisation (IVF) treatment is not common in most public hospitals but is gaining popularity in the private sector. A dual practitioner who specialises in IVF treatment may use more government time due to a large private clientele. The same is applicable to obstetrics and gynaecology specialists. Competition for time may affect public patients more than private ones. The availability of DP physicians may be greater in private practice than in the public-sector workplace. Again, dual practitioners who specialise in certain areas of medicine- for example, work affecting women and children- may be more susceptible to the abuse of government time, given their large patient clientele.

One possible intervention available is to upscale the monitoring of doctors' attendance, as has been done in Peru (LaGrone, 2017), where doctors have been subjected to fingerprinttaking. The use of technology such as fingerprint-recognition devices can be used to record or track doctors' attendance.

\subsubsection{Stealing /outflow of government resources}

The debates on dual practice have suggested that public/private practice may bring about the pilfering of public hospitals' resources, as well as under-the-table payments (Ferrinho, et al., 2004b; Ferrinho et al., 2004a). Dual practitioners may also use the government's supplies and equipment to treat patients in their private facilities (Eggleston \& Bir, 2006; Jean \& Sass, 1995). In Kenya for instance, Anuro (2014) shows that large quantities of drugs stolen from government hospitals are diverted to private practice or sold on the 'black' market, and 
despite the importance of the anti-rabies drugs, health care providers in government hospitals were stealing them to sell at a higher cost in the private sector. Similarly, in Costa Rica, Panama and Venezuela (Ferrinho, et al., 2004b), in Malawi, (Muula \& Maseko, 2006) cases of theft of public health facility equipment and materials have been reported. In Nigeria, anecdotal evidence shows that this happens, although, this may be difficult to assess. Evidence from Ethiopia also reported that evidence of drug stealing from the public sector due to private practice was problematic (Abera et al., 2017). However, more generally, public resources- such as in-house hospital training, seminars and conferences organised in the public sector, as well as knowledge acquired from the public sector are transferred to the private sector indirectly. These transfers constitute losses to the public system.

The tendency to steal resources may not be peculiar to dual physicians alone. However, dual physicians who run clinics may be more susceptible to pilfering hospital resources, as those products can easily be used in their private clinics. Inasmuch as anecdotal evidence is insufficient, more empirical evidence is needed to substantiate such claims.

\subsubsection{Dual practice and health care costs}

Dual practice may increase the cost of health care in both private and public sectors (González \& Macho-Stadler, 2013; Socha \& Bech, 2011). First, it is assumed that the dual practitioner may intentionally over-treat patients to gain reputation and boost his or her private practice. It is also alleged that over-treatment helps DP doctors to charge a higher fee-for-service (FFS) for unnecessary treatment. For example, when a physician is known for giving good treatment and curing patients to their satisfaction in the public sector, the reputation gained may increase patients' utilisation of his/her private practice. However, this is not without a cost. In the public system where there is a third-party payer, the social cost may be borne by the system, and this may reduce the welfare gain due to the over-treatment cost. Additionally, in a healthcare system where payment for health services is fee-for-service, as it is in most developing countries including Nigeria, there could be under-treatment, which means that doctors have a laissez-faire attitude, whereby patients who are not willing to make extra payments are left with minimal, and often inadequate treatments, and the onus is on the patient rather than the doctor to ensure that everything necessary is done. This attitude indirectly persuades patients to visit the private clinic. 
In Nigeria, the cost of over-treatment is borne by the patient, although in private practice the practitioner gains the reputation and the financial reward in the form of fee-for-service. This happens in the form of provider moral hazards, basically to achieve the best possible outcome and to increase the cost of care. However, this intention may be suppressed, depending on the socio-economic status of the patient involved. The provider may not have an interest in overtreating poor patients if they cannot pay. But if the over-treatment is on moral or ethical grounds, and the poor patient achieves over-treatment with the highest possible outcome, the provider's reputation will be advertised by the patient. Patients who are beneficiaries of this provider's largesse will help to spread their reputation.

Second, some dual practitioners may allow deferred payments to reduce the payment burden on patients (Onah \& Govender, 2014). For example, in Nigeria, private providers allow patients who would not otherwise be able to afford a one-off payment for their treatment costs to pay in instalments. In this way, it may be possible to believe that dual practice could help service users to reduce their financial access burden. This is also a strategy to boost their reputations and is hardly ever allowed in the public system.

Nevertheless, past studies have not shown the estimated cost of private treatment when patients are diverted to the private sector compared to the estimated cost of treatment in the public sector. In the same vein, there is no evidence on the total financial loss to the public system of absenteeism, truancy and late reporting to duty by dual practitioners. This evidence is crucial for any reform on dual practice. This, in part, is what this study sets out to achieve.

\subsection{Gaps in knowledge}

Currently the literature on benefits and disbenefits reveals both positive and negative impacts of dual practice, so that it is difficult to reach a conclusion on the overall balance of advantage. For example, absenteeism, misappropriation of public resources and long-waiting times may all be associated with DP, while on the positive side extra private provision increases health system capacity and may sometimes bring good training opportunities. The review identified a lack of evidence on the estimated losses to the public sector due to paid duty hours that dual practitioners did not work. Also, the literature is scant on the estimated 
cost to patients when they are transferred from the public sector to the private sector, and the coping mechanisms for paying for private treatment. Information on these topics is needed to inform the debate on DP policy reform.

\subsection{The extent of physician self-referral}

\subsection{Introduction}

In this section, we review the literature on patient diversion from public to private sector. The extent and patterns of diversion are examined to determine whether this is common practice.

\subsection{Findings regarding diversion}

Patient diversion is described as a situation where physicians on a full-time public contract divert patients from public to private hospitals where they have direct or indirect financial interests. When a physician diverts patients to receive health care services in a facility that the physician owns, leases, or has a financial interest in, with a commission per case referred, the practice is called self-referral (Hughes, Behargavan, \& Sunshine, 2010).

Self-referral can take two common forms. A physician who is not trained as an imaging specialist but is sending patients to his or her own on-site imaging services can be termed to be engaged in self-referral. Typical examples might be obstetricians or their staff performing ultrasound examinations or internists performing and interpreting chest radiographies. The second form of self-referral may involve physicians referring their patients to outside facilities where they have a financial interest (Kouri, Parsons, \& Alpert, 2002).

In some countries, financial interest may be in the form of a commission paid to the selfreferring physicians for referring patients to places where they derive financial benefits. In Nigeria for instance, physicians do privately refer patients for diagnostic tests in certain designated centres and insist that the laboratory test must be done in those places or else the test may not be accepted by the self-referring physician. They receive a commission on any referral made to the diagnostic centres. In most cases, the physician may not be the owner or a joint-owner of the diagnostic centre, but they engage in the practice for either patient welfare 
or a financial gain. This calls for concern as the patients seem deprived of power in making the choice of laboratory centres or hospitals to attend. In Nigeria, it seems to be commonplace for specialists employed in government health facilities to refer public patients to their private clinics, but whether this practice is borne out of professional altruism or for financial gain remains a matter of controversy. The consequence of this practice in health systems is worth investigating.

\subsubsection{Dual practice and the extent of self-referral}

Evidence has shown that the diversion of patients to the private sector occurs in countries such as Peru, Zimbabwe, Bangladesh, India, Portugal, and the UK (Garcia-Prado \& Gonzalez, 2011). Similarly, Mossialos et al. (2005) reported on the Greek doctors' transfer of patients from the social insurance funds where they work part-time to private practices. The public/private mixed system seems to offer the incentive for public patient transfer such that those with elective cases that require long-wait-times may have to seek private care (Cheng, Haisken-DeNew, \& Yong, 2015). The motives for such transfer arguably may be due to long wait-times or delays in the public sector, but the overriding influence seems to stem from a complex mix of motives in a professional group that sees it as normative to straddle both sectors (Humphrey \& Russell, 2004). The transfer of public patients may have both benefits and negative consequences for both patients and the public system. Those who can afford private treatment may opt out of the public sector and pay more or use private health insurance, while those who cannot afford it, but are coerced through diversion, may find it difficult to cope with payment. The cost to the public system of patient diversion needs to be estimated to establish the revenue loss from the public system due to diversion. The question, nevertheless, is whether there could be a trade-off between the reduced revenue for government hospitals due to patient diversion and an increased cost and the timely access to private treatment for diverted patients.

Past studies have shown that the physicians' ownership of health facilities may increase an over-utilisation of such services. For instance, Mitchell \& Scott, (1992) found that the physician ownership of health facilities was associated with the higher utilisation of three important services. Thus, in the case of clinical laboratories, there was a $94 \%$ increase, with diagnostic imaging having a 5\% to $65 \%$ increase in scans per 1000 population, and with 
physical therapy displaying a $43 \%$ increase in visits per patient. Another study by Swedlow and colleagues found that physical therapy for musculoskeletal injuries was ordered 2.3 times more by self- referring physicians than by doctors who had no stake in the facility involved (Swedlow, Gregory, Smithline, \& Milstein, 1992) .

The complexity of the health care market shows that both consumer and provider moral hazards affect the market (Yawson, Biritwum, \& Nimo, 2012). While the provider has the tendency to over-treat for financial self-interest, of which the cost may be borne by a thirdparty payer or the private patient, the user may intentionally demand frivolous health care services, which is a loss to the third-party payer or the system. On the other hand, patient diversion may be a response to a system that seems to have neglected the autonomy of the medical profession and reduced them to ordinary civil servants. It is important for the government to create a manageable interface that can increase the doctors' performance in the public sector by mounting contract-hour surveillance on their activities. The author argues that there is a need to generate the cost to patients of private treatment when diverted and their self-assessed coping mechanisms.

\subsubsection{Self-referral and cream skimming}

In Australia, the cream skimming of wealthier patients and those with less complex cases from the public system to the private sector has been reported (Cheng et al., 2015). It has been shown that dual physicians self-refer patients with less complex case mixes to their own clinics, while the more severe ones are sent to general hospitals where they are reimbursed through the Prospective Payment System (PPS) (payment to hospital in advance). There are a number of studies that have shown evidence of the cream-skimming of patients. According to Mitchell (2005), physician-owned centres treated proportionately more minor surgical cases compared to non-owners, and patients with fewer co-morbid conditions. The "sorting" of wealthier patients to providers with private practices in Indonesia is well documented (Rokx et al., 2010). The sorting process was said to occur through the market and direct encouragement to richer patients by dual practitioners for them to seek private treatment. A study by Winter (2003) has also shown that patients with more medically complex conditions tend to receive treatment at hospital outpatient centres instead of ambulatory surgical centres (ASCs). ASCs are owned by physicians or through a joint venture with hospitals or 
corporation. The allegation against dual practitioners of diverting more healthy patients to their private clinics may imply pushing more costly cases to the public system. This may mean treating complex cases at a reduced cost in the public system and avoiding costlier private treatment, which might be unaffordable for poorer patients. In a way, this could be beneficial for poor patients. While diversion may provide quicker access to private treatment, there is a need to estimate the cost implications for patients of different socio-economic groups when they are diverted to the private sector and to establish the other characteristics of patients targeted for diversion.

\subsubsection{Dual practice and indirect patient diversion}

One of the criticisms of dual practice is the indirect means used to divert public patients to the private sector. Indirect referral strategies include reducing the quality of service, inducing waiting periods or waiting lists in public hospitals (Kiwanuka et al., 2010) or induced referral (Garcia-Prado \& Gonzalez, 2011). This seems to be dependent on the rigidity or fluidity of the interface between the NHS and private work (Humphrey \& Russell, 2004). If the interface arrangement is very strong, the dual practitioner may not have the opportunity to influence the work process, but if relaxed, as it is in developing country context, they can shirk responsibilities and exert a considerable influence on the work process.

The issue of reducing quality in the public sector as an indirect self-referral seems to be a valid argument when considered against the central role of the doctor in the quality of patient experience. For instance, evidence from India shows that dual practitioners spent more time with patients, completed more items on a checklist, and were more likely to provide a correct treatment in the private sector in comparison with the public sector (Das et.al, 2016). Thus, the dual practitioner does have an overriding influence over elements of quality experience and in some instances could also play a part along with other providers.

In Ethiopia, a new form of patient diversion was reported (Abera, et al., 2017). Public patients initially diverted to the private sector were later redirected back to the public system without receiving treatment in the private sector, a term referred to as circular diversion pattern. This is often the case in many developing countries where patients diverted to the private sector are sometimes kept for a short or long stay before being referred back to the public sector for 
specialist attention. This kind of predatory behaviour benefits the practitioners financially, which means that the patient would have spent a substantial sum in the private sector before they were returned to the public sector.

\subsection{Gaps in knowledge}

Currently, the extent of patient diversion by public-sector doctors is not known in Nigeria but is important to generate evidence on the number of public patients diverted at a given time, the ways of diverting them, the financial cost to patients when diverted and the financial loss to government hospitals.

\subsection{The motives for engaging in dual practice}

\section{$3.10 \quad$ Introduction}

Understanding of the motives for private practice by public doctors provides an important base for any reform of dual practice. The motives for dual practice vary across health systems. It may be difficult to make an effective effort in regulating dual practice without first investigating why public doctors engage in private practice. It therefore, remains crucial that the motive is understood as a starting point for the design of regulatory measures. At the same time, the motives of public sector doctors who work only for the government and are not engaged in private practice also warrant investigation to provide a point for comparison. In every health system, the nature and characteristics of the health market may help shape motives for engagement in extra income-generating activities by public doctors.

\subsection{Findings regarding motivation}

In this section, a review of the literature on motives for dual practice was carried out. The common motives for dual practice among government doctors are grouped under the following sub-headings. 


\subsubsection{Poor remuneration}

This seems to be one of the major motives for engaging in dual practice across health systems. Past studies have overwhelmingly identified poor remuneration as a major tracer for dual practice (Akwataghibe, et al., 2013; Ferrinho, et al., 1998; Humphrey \& Russell, 2004; Roenen, Ferrinho, Dormael, \& Conceicao, 1997; Russo, McPake, Fronteira, \& Ferrinho, 2013b). This extra income can then be used to support families. For instance, it has been shown that one month's public-sector salary is equivalent to seven hours of private practice in certain Portuguese-speaking African countries (Ferrinho, et al., 1998). In Guinea Bissau a doctor's salary barely pays for house rent (Roenen, 1997), and although the study did not specify the category of doctors in question, it nevertheless seems too little to motivate a doctor. In Peru also, dual practice is being attributed to the underlining pressure due to financial need (Jumpa, et al., 2007). The standard salary for a Chinese doctor in Guangzhou city seems too low, at a basic salary of $1300 \mathrm{RMB} /$ month excluding bonuses (Ran, Luo, Wu, Yao, \& Feng, 2013). This is equivalent to $£ 147$ per month. In Nigeria, for example, a consultant on grade level 6, step 9 earns a monthly gross income equivalent to $£ 980$, excluding other allowances.

Similarly, NHS doctors in the United Kingdom regard financial incentives as the major factor for engaging in both the public and private sectors (Humphrey et al., 2004), but consultants earn between $£ 76,761$ and $£ 103,490$ per year (HealthCareers, 2017). This means that a consultant's monthly salary in the British NHS may be able to pay for six consultants' salaries in developing countries.

It is surprising that poor remuneration seems to be the common denominator across different health systems. Considering the Purchasing Power Parity (PPP), doctors in developed countries are at a considerable advantage comparatively. The implication, however, particularly for doctors in LMICs, is that extra income from private practice seems unavoidable. Overall, poor remuneration may not be the major motive for DP in all health systems. This has been identified in the work of (Dieleman, Cuong, Anh, \& Martineau, 2003), which shows that the major motivating factors for health workers are appreciation from managers and community, stable job and income and training. On the other hand, low salary was identified as one of the main discouraging factors, by and large; an extra income may 
stand out as fundamental (Lerberghe, Conceic, $\mathrm{a}^{\sim}$ o, Damme, \& Ferrinho, 2002). In the same way, an assessment of service motives and profit incentives among physicians in Norway found financial incentive to be an important marker for physicians, but not sufficient to determine their behaviours (Godager \& Luras, 2009).

\subsubsection{Confers credibility, prestige, and job security}

Public service work is seen as conferring credibility, prestige and job security by doctors who use it as a platform to extend their private practices (Roenen et al., 1997; Biglaiser \& Ma 2007; Gonzalez, 2004; Eggleston \& Bir, 2006). Some doctors may see public work as an advertisement for their private practices. People know that doctors employed in the public sector are qualified and well trained and that the government cannot compromise on recruiting only qualified medical professionals in public hospitals. In reality, doctors in tertiary hospitals in Nigeria are respected for their qualifications and experience. The respect they derive from public work may help to advance their private practice. For instance, in Bangladesh many young doctors regard public hospital work as a stepping stone to private practice, unlike the older doctors, who need not rely on government work to develop their private practices (Gruen, et al., 2002). This suggests that some dual practitioners may use government hospitals to increase their reputation for private practice. Traditionally, in the UK, unpaid work in voluntary hospitals (i.e. pre-NHS) was necessary to make contacts and get private referrals from the younger doctors one had trained. Even today, an NHS contract may be important to establish status and is required by some Primary Medical Insurance (PMI) providers before they will pay for private treatment via a consultant.

On the other hand, doctors' reputations may increase with private work, as has been shown in Cambodia, where dual practice was found to enhance a strong professional reputation, job security and career progression (Henderson \& Tulloch, 2008). In the same vein, high earnings and prestige may be regarded as pull-factors encouraging qualified and experienced doctors to engage in dual practice (Nitararumphong, Mills, Pongsupap, \& Tancharoensatien, 2005).

Besides, employment in the public system is relatively more guaranteed than in the private sector. In Nigeria, for example, there is no pension scheme in the private sector; neither is the NHIS covering those in that sector. The rights and privileges of doctors are more often valued 
in government work. For instance, when the Croatian government banned private practice in 1994, the majority of doctors decided to remain in the state hospitals for fear of losing their position and not being able to survive in private practice (Gabric \& Lazic, 2013). Although private work may look attractive, in terms of financial gain in the short term, public work ensures a regular income and a more guaranteed future for doctors in Nigeria.

\subsubsection{Autonomy}

The autonomy of doctors seems to have been eroded with public work. This is because it tends to reduce them to civil servants with overwhelming rules that may sometimes conflict with professional ethics. There is a degree of strategic control, which public work does not allow government-employed doctors to enjoy, except in the private sector (Humphrey \& Russell, 2004). Even in the private sector, the extent of the autonomy may be limited. A previous study by Waring and Bishop (2012) on the Independent Sector Treatment Centres (ISTC) involved reduced autonomy because doctors must follow strict protocols governing the treatment of large volumes of similar elective cases. Doctors may engage in private practice where they can use their initiative to gain control over their work space and patients. In Nigeria, bureaucracy in government hospitals is said to affect doctors' clinical decisionmaking power and limit their judgements. In Finland, it has been shown that a chance to apply one's own ideas is an important source of job satisfaction in private practice (Kankaanranta et al., 2007), although financial gain still stands out. In private practice, the doctor is not under any management or bureaucratic rules but makes decisions on his patients' management straight away. In this way, there is status recognition among dual practitioners in their private practices, and opportunities to practice new skills, which may not be available in the public sector.

\subsubsection{Professional skills development}

Previous studies have found that dual practice has the potential for professional or skills development (Berman \& Cuizon, 2004; Humphrey \& Russell, 2004; Jamshidi \& Reilly, 2008). Private practice may provide the opportunity for skills acquisition from the diversity of case mix. Some may see private practice as an important source of additional patients with diverse conditions that could enable them to gain experience more quickly (Humphrey \& 
Russell, 2004). A study on doctors' choice of workplace in Finland shows that career and professional development were found to be two highly ranked motivating factors (Heikkilä, et al., 2014).

Resident doctors particularly may find skills acquisition useful in the private sector through the maintenance of clinical skills. Motivation for moonlighting was attributed not only to financial benefit but to the development of clinical skills (Jamshidi \& Reilly, 2008). In LMICs with a large private market, the importance of gaining experience in both sectors cannot be over-emphasised. The public system may lack modern equipment for diagnosis and treatment, which may be readily available in the private sector. However, beyond the financial motive, additional knowledge and experience may be obtained in private practice. In Nigeria, for instance, the best neurological service is obtained in the private sector and is accredited by the medical college in the country to offer training to medical students.

\subsubsection{Growing demand for private health care}

Tracing the growth of private practice in Sweden (Mariynn, 1992) has shown an increase in public interest in having the choice of where to seek medical care - this freedom of choice increased patronage for the private sector. Given the growing criticism about long waiting times in the public sector and an increase in public interest to seek medical care outside the public sector, there were a growing number of doctors with the financial motivation to join private practice. The private sector in Sweden accounts for $25 \%$ of the coronary bypass surgery performed through contracts let by the purchasing county councils to private doctors. In Denmark, there has been an appreciable increase in the use of private hospitals since 2005 and in 2010 the approximate bed capacity was 500, accounting for $2.5 \%$ of all hospital beds (Olejaz et al., 2012). Similarly, in the UK, the NHS has always existed side by side with the private provision of care. It has been shown that the private health care market may reach an annual growth rate of $2.8 \%$ by 2025 (Perryman, 2017). The size of the UK private sector is expanding rapidly. In 2015 , private acute health care stood at $£ 7.8$ billion and 3.94 million private health insurance policies (Royce, 2009) It is suggested that the waiting list in the NHS seems to be driven by the surge in self-pay for some medical procedures. Patients who can no longer endure the long NHS queue and can afford private treatment move to the private sector. 
In the developing country context, the size of the private market has always been larger than the tax-financed public system. In fact, it has a large and expanding role to play in the health systems of LMICs (Horton \& Clark, 2016). In Nigeria, for instance, private expenditure on health accounted for $74.9 \%$ in 2014 (WHO, 2016). This is due to the neglect of the public system, accentuated by poor resources and governance failures of the public sector. The current commercialisation of health care, though, an alternative to public health care, seems to attract many public-sector physicians who perhaps are responding to the sharp increase in the sector for financial reward.

\subsection{Gaps in knowledge}

The literature suggests that there are a variety of motives for DP with extra financial income often seeming to be a key factor, but it is unclear what precise combination of motives applies in a developing country, such as Nigeria. There is also a dearth of information on why many mission-oriented public doctors who are on full-time government contracts do not engage in private practice.

\subsection{Quality of healthcare in the public sector compared with private practice}

In this section of the literature review, an attempt was made to provide a background for healthcare quality, and to provide a definition. The review examined previous studies on the concept of quality in healthcare. Some of the different benchmarks for measuring quality were discussed. Furthermore, there was a comparison of quality of healthcare in the public and private healthcare sectors. However, there is scant number of published studies that specifically compares quality of healthcare across public and private practices of the same doctor.

\subsection{Background}

The concept of quality is crucial for policy makers, service users, and managed healthcare organizations. Institutions and governments that provide financial resources could design tools to monitor resources they invest in hospitals to measure performance and level of client 
satisfaction. Although, it may not be easy to produce an error-free measure of quality, nevertheless, it has been suggested that the sources of information to be used for quality measure have to be credible (Brook, McGlynn, \& Shekelle, 2000).

The Institute of Medicine (IOM) has defined quality as "the degree to which health services for individuals and populations increase the likelihood of desired health outcomes and are consistent with current professional knowledge" (IOM, 2001). According to IOM, the final test of quality of a health care system is its ability to help the people it is designed for. Quality is important to both patients and healthcare providers. It can be used by service users to influence preferences and choice of which provider to visit, while for providers, it can help to retain existing customers and attract new ones as well.

Today, the business of monitoring quality is shared among stakeholders in the health system - the professionals, service users, policy makers, and government. The service users are aware of the type and quality of services they would want from healthcare providers. Those who purchase and pay for services on behalf of the population are also aware of what quality and type of services their beneficiaries would want to receive. So, quality of care can be assessed at different levels - from the care provider to service users - and those who purchase services on behalf of others (Brook, et al., 2000).

Quality is a complex phenomenon with different meanings for different individuals. It has been shown that treatment can be customised for different individuals with similar illnesses. For instance, medicine is full of procedures and tests, which can either be beneficial or harmful (Brook, et al., 2000), but how to minimise the harm matters a lot. The ability to measure scientifically what healthcare providers do will help to increase the level of trust the society has on healthcare providers as well as the health system. The concept of 'one-size-fitsall' may not be applicable in healthcare, due to differences in individuals' health needs. But how healthcare consumers conceive quality of care received remains an important issue to be explored. 


\subsection{Definition of quality}

There seems to be no consensus on what constitutes quality in healthcare (Donabedian, Wheeler, \& Wyszewianski, 1982; Maxwell, 1984; Peters, 1987). Availability and accessibility are two crucial variables, which define healthcare quality. However, availability of health services may not provide detailed information on the nature of services provided and if the services are adequate or not,

or whether it satisfies the service user (Bruce, 1990). Financial cost has been suggested to be an important element of healthcare quality evaluation (Donabedian, 1997). Lower quality may be attributed to low cost of healthcare, but costly healthcare may not necessarily be high quality care.

Moreover, service quality in healthcare is viewed as the difference between customer expectations and customer perceptions (Parasuraman, Zeithaml, \& Berry, 1985). While 'expectation', connotes what the customer feels the provider should offer, 'perception, refers to customers' evaluation of the service provided. Customers stand at a vantage point from which to evaluate how services are received from a provider. They can judge if provider A can professionally meet their expectations better than provider B, and even if they lack the knowledge to judge as an expert might their perceptions are likely to influence their future behaviour.

Access and effectiveness have been suggested as quality indicators (Campbell, Roland, \& Buetow, 2000). Access to health care may not necessarily mean availability, but rather the potential to use the available service (Gulliford et al., 2002). Effectiveness, on the other hand entails an evidence-based care that is proven to be better than the alternative (IOM, 2001). Thus, Campbell and his colleagues argued that quality of healthcare has meaning when applied at the individual level only insofar as the individual is the recipient of care. Donabedian included in his quality care definition, attributes such as, efficiency, effectiveness, efficacy, equity, acceptability, and legitimacy (Donabedian, 1980). Similarly, he argues that the evaluation of healthcare quality has meaning only when it applies to healthcare consumers who are definers of quality (Donabedian, 1992). In the same vein, Flood, Scott, Ewy, \& Forrest (1982) see quality as meeting customers (agreed) requirements, 
formal and informal, at lowest cost, first time every time. This seems generic and faces operationalization challenges. At the extreme, poor quality can be detected by obvious patient neglect as reported in the Mid Staffordshire NHS Foundation Trust (NHS Foundation Trust, 2013), and measures such as high mortality rates of which, at least 44,000 and perhaps as many as 98,000 people, die in hospital each year due to preventable medical errors (Kohn, Corrigan, \& Donaldson, 1999). The Institute Of Medicine (2001) has come up with six aims of quality improvement, which include safety, effectiveness, patient-centeredness, timeliness, efficiency, and equity. Whether these quality measures are observed in the health system remains debatable, but many experts believe, they set the objectives for the health system to strive for.

The quality literature is so extensive that a full review is beyond the scope of this thesis (but see, Sale 2005 for a useful summary). Quality can be divided into technical and functional subcategories. Technical quality refers to accuracy and procedure, which basically encompasses the competency of staff and their compliance with guidelines on service delivery. This may be difficult for the uninformed service user to detect (Bowers, Swan, \& Koehler, 1994). It has been argued therefore, that the quality of technical care from the standpoint of the consumer should not be what is done (process), but what is accomplished in terms of final product (Donabedian, 1992). Outcomes are more visible to the consumer than process. The functional aspect of quality refers to the 'how' or 'process' by which services are delivered to the service users. However, it has been argued that service users will often pay more attention to factors such as physician reputation, availability of equipment such as an MRI scanner, building aesthetics, food served and attitude of staff - than to clinical effectiveness in terms of measured treatment outcomes (Royce, 2009). Thus, service users may perceive quality in different ways at different levels - for example in terms of the actual treatment service received, physical environment, and interaction with service providers.

\subsection{Assessment of quality}

Several indicators have been used to measure quality (Swan \& Boruch, 2004). International comparison and benchmarking have shown that there is a problem in establishing universal comparative measures of healthcare quality (Raleigh \& Catherine, 2010). Similarly, the 
Organization for Economic Co-operation and Development's (OECD) Healthcare Quality Indicator Project (Arah, Gert, Jeremy, \& Niek, 2006) is a comparative initiative covering many OECD countries, and with a number of quality indicators. However, it was found inadequate for a cross-national comparative analysis in such areas as data availability, methodology, and interpretation (Mattke, Epstein, \& Leatherman, 2006). Additionally, a comparative analysis of quality may be difficult due to different national approaches to data storage, differences in the statistics used, and differences in the way patient groups are classified.

Furthermore, the application of broad-based measures of service quality based on patients, staff and organizational expectations such as SERVQUAL (service quality), also have problems. Critics contend that SERVQUAL neglects patient/provider interaction and ignores outcome measures such as recovery from pain, saving life, or feelings of remorse after receiving care (Ramsaran-Fowdar, 2005). Therefore, SEVIQUAL may not be an appropriate measure for technical quality because it excludes service customisation and professional knowledge. Similarly, patient satisfaction measures are fraught with subjective assessment of what happened in the process of care, which may not be directly associated with quality (LaVela \& Gallan, 2014). It is argued that patient satisfaction is mainly affective judgment of patients' experience and how they feel and do not constitute any quality measures (LaVela \& Gallan, 2014).

According to Campbell, two processes of care are often identified. These are technical skills, and inter-personal interactions between users and providers (Campbell, 2000). Technical skill refers to the application of clinical medicine to the individual health problem. Inter-personal skill is necessary in the management of patients. It includes communication with patients, the ability to build relationships between carers and service users by showing empathy.

One of the most widely used quality measure is the Donabedian quality assessment measure, which comprises the components of structure, process and outcome (Donabedian, 1997). The Donabedian approach to quality assessment has been used in several studies (Brook, et al., 2000; Campbell, et al., 2000; Larry, Jeffrey, Alexander, \& Joanna, 2008). According to Donabedian, healthcare quality can be classified under three categories: structure, process, 
and outcome. Structure describes the physical attributes where care takes place. This includes material resources such as buildings, equipment, human resources, which include personnel (number and qualification of personnel) and organizational structure (medical staff), and method of peer review.

Process measures describe what is done in the process of receiving care. This involves the information the patient gives out and the activities/diagnosis the provider carries out based on the information received, recommendation and implementation of treatment (Donabedian, 1997). It includes the interactions between users and healthcare providers. It is the service user's expectation that defines what is accessible, convenient, comfortable and timely (Donabedian, 1992). In addition, other assessments of quality care have incorporated patient experiences of care in nine key areas: communication with nurses, responsiveness of hospital staff, pain management, communication about medicines, discharge information, cleanliness, and quietness of hospital, overall rating of the hospital, and patient willingness to recommend the hospital (Thomas, Zazlavsky, Cleary, \& Landon, 2010).

Patient report measures have been argued to have several advantages over technical measures. First, the experience about care is easier to understand for patients than technical measures, which patients may not know (Wilson et al., 2007). This is supported by Bruce (1990), who argues that clients do not see the organization behind the services provided, neither are the apparatus used for care discernible to them in the care process. But their focus, however, is more on the outcome of services rendered.

Outcome is the effect of care on patients' health status as a result of care services received. In other words, it is the consequence of care. This suggests that patient expectation is to recover from treatment received. If this expectation is met, the patient may become satisfied with services, and may rate the quality of service received higher.

This three-part approach for quality assessment can be linked together, and the existence of one may enhance the functionality of the other. For example, the existence of good structure may increase good process, which may enhance patient's outcome. However, each element in this approach may vary depending on situation, and a combination of any two or three of them cannot be linear (Campbell, et al., 2000). It has been shown that the combination of any 
two of these variables may produce different results depending on the level of analysis (Larry, et al., 2008). Moreover, there is no firm causal link according to Donabedian, good care can have bad outcomes - there are risks. For instance, structure and process can be defined in their abilities to result in improvement of health status based on user evaluation. This implies that a combination of clinical capacity (medical), and non-clinical (process) can help to inform the user's subsequent decision to seek consultation or not (Rogers, Hassell, \& Nicholaas, 1999).

Although the combination of these elements may lead to a more rounded approach to evaluation, both process and outcome measures may be suitable for quality assessment; nevertheless, process indicators could be better than outcome measures when the aim of measurement is to influence the behaviour of healthcare system (Campbell, et al., 2000; Davies \& Crombie, 1995). This is because the activities under care process are common and more visible to record and measure. On the other hand, outcomes may be poor measures of quality of care, and may depend on a range of other external factors beyond the control of the provider (Giuffrida, Gravelle, \& Roland, 1999). For instance, in the case of an emergency, late visit to a provider, terminal illness, consumers' expectation about outcomes are rarely realised. However, the proof of the suitability of process measures could be determined by the evidence for improved outcomes.

However, outcome measures have been criticized in some respects. Though, patients expect health outcomes when they seek healthcare from providers, those expectations are rarely met, and are often influenced by certain external factors such as age, educational background, or natural history of the disease (Campbell, 2000). A study that evaluated service quality and patient satisfaction of primary health care services in Southwest Nigeria showed that patients' expectations were not met by the five quality-tracers used in the study (Adepoju, Opafunso, \& Ajayi, 2018). This implies that frequent failure to meet expectation may lead to a lowering of expectations since experience of the service is one of the determinants of expectations.

Thus, it is suggested that where outcome is used as a tracer for quality, there is need to adjust for case mix and external factors to standardise comparisons across providers and health facilities. Another important drawback for the use of outcomes to measure quality is that 
many of the most important real-world outcomes may occur years later, patients, therefore, can be biased, and may give such a quality measure low importance. Even though, outcome may be delayed, it has the advantage of showing the contributions to care from structure and process elements, including from patients who can say what went right or wrong in the process of care.

Regarding the structure measures of quality, it is not conclusive how these can help to enhance the function of other elements of quality measurement (Brook, et al., 2000). For instance, no evidence has shown that physical buildings, availability of financial resources, or hospital accreditation, can determine what sort of care a patient receives from a provider (Brook, et al., 2000).

Notwithstanding the above arguments, Donabedian (1997) has argued that assessment of quality of care should include the three elements as their inclusion will allow supplementation of weakness in one approach by the strength in another. His argument shows that healthcare consumers have important roles to play in quality assessment, that the purpose and direction of quality assessment is anchored on patient judgements.

\subsection{Comparing the quality of private and public healthcare}

There is limited literature that compares quality of care in the public sector with quality of care in private healthcare managed by dual practitioners; however, evidence from India shows that dual practitioners spent more time with patients, completed more items on the checklist, and more likely to provide a correct treatment in the private sector in comparison with the public sector (Das et.al, 2016). Another available evidence shows that when providers offer the same services in both public and private sectors, there is an incentive to offer low quality service in the public sector (Berman \& Cuizon, 2004). The declining quality of care in the public healthcare is often attributed to a decreased personal availability of public doctors (Ferrinho, et al., 1998). However, there is a paucity of empirical evidence to support such comparisons. This is relevant to this study, which uses a comparative design.

It has been argued that dual practice improves quality by incentivising dual practitioners to give more attention to accurate diagnosis and quality to attract to themselves respect and 
prestige for future private work. But for those public-sector doctors who do not engage in dual practice, simple logic would suggest that they would provide higher quality in public facilities since they do not have a competing interest with private sector work. However, there could be differences in quality among individual doctors, but the overall quality at hospital level may remain low due to competing interest between public and private sector engagement.

A comparative study of public and private hospitals shows that private facilities perform better than the public for drug availability and quality of service (Gilson, Alilio, \& Heggenhougen, 1994; Sima, Heywood, Sandy, \& Garner, 2011). Additionally, there seems to be an average longer consultation time in the private sector than the public (Kanji, Kilima, Lorenz, \& Garner, 1995; Pongsupap \& Van Lerberghe, 2006). But it can be argued that the public sector often records more patients than the private sector, therefore, it will spend shorter time with patients than in the private sector. Again, administrative and bureaucratic rules may influence wait-time in the public sector, which may not be under the doctor's control. Overall, a balanced comparison of the two sectors may depend on context.

On technical quality, the private sector has performed less well than the public. The public is often perceived as being competent, though, it has bureaucratic hurdles that often discourage service users, and has also been accused of over prescription, and moral hazard (Russel, 2005). On the other hand, the private sector has been criticised for over prescription and use of fake drugs (Hoa, Ohman, Lunndborg, \& T., 2007; Lindelow \& Serneels, 2006).

On the issue of accessibility, the private sector provides a higher number of outpatient services (Basu, Kishore, Panjabi, \& Stuckler, 2012). For instance, it is estimated that more than $90 \%$ of children affected by diarrhea in India are taken to private healthcare, and in Vietnam, $60 \%$ of all patient contacts take place in the private sector (Bustreo, Harding, \& Axelsson, 2003). It may not be conclusive that access to private hospital is more than that of the public; however, there are many factors that determine access. For instance, wait- time is shown to be shorter in the private than the public (Basu, et al., 2012). In Nigeria also, private facility may be preferred among rural dwellers due to shorter wait-time (Brugha \& Pritze Aliassime, 2003). 
Others have reported disrespect and lack of hospitality by public providers, (Gilson, et al., 1994). There is also recorded poor quality with unqualified personnel resulting in poor diagnostic accuracy and medical management in the private sector (Basu, et al., 2012).

Nevertheless, public sector outcome is found to be positive. There was a higher success rate in the treatment of tuberculosis and HIV/AIDS as well as vaccination (Soeung, Grundy, Morn, \& Samnang, 2008). In contrast, private provision of TB services was found to be poor due to non-prescription of the WHO recommended TB regimens in Thailand, (Chengsorn, Bloss, Anekvorapong, Anuwatnonthakate, \& Wattanaa-mornkiat, 2009). Furthermore, studies on users' perception of care showed a lack of consensus on which of the providers - private or public - has a better quality of service. While some patients prefer the private sector for better quality, others hold that public providers have more technical skills (Siddiqi, Hamid, Rafique, \& Ali, 2002). This might have to do with their psychology — having paid; they must believe it is better quality.

This study, therefore, applied the Donabedian three elements of quality measures — structure, process and outcomes - to measure quality in dual practitioners' private care and the publicsector care. This is because the combination of these elements can provide more information on healthcare quality rather than using any one or two categories.

\subsection{Gaps in Knowledge}

The area of primary concern in this thesis is how Nigerian service users and other stakeholders perceive quality and how far this shapes their decisions about using DPs. Existing studies do not give a clear picture of the extent of quality differences between public and private sectors and how these are perceived. There may be a difference in the quality of service the DPs render in public and private settings, but there is little evidence on this point from Nigeria. Indeed, the literature contains little in the way of systematic evaluation of quality in the two types of settings in any developing country. 


\subsection{Regulatory mechanisms for dual practice in different health systems}

This section presents background information on regulation, and actors involved in regulating dual practice. It critically examines various regulatory mechanisms/options for dual practice and their challenges in different health systems.

\subsection{Findings on regulating DP}

Generally, regulation comprises policies, laws, and rules developed by a government or professional association aimed at restricting or abolishing conduct or practice that counters its target or intention. Regulation includes the use of administrative control by a government to correct market failure (Ensor \& Weinzierl, 2007). It is widely agreed that regulation is an instrument used by a government to control what individuals or firms do to achieve their objectives (Hongoro \& Kumaranayake, 2000). Specifically, government can use regulation to target variables of interest such as prices, quantity, distribution and quality of a product (Kumaranayake, Lake, Mujinja, Hongoro, \& Mpembeni, 2000). Regulation can serve the purpose of protecting consumers as suggested by Mills (2002) to seek redress when they are victims of medical malpractice, or, creating a level playing ground for competitors.

Regulation according to (Kumaranayake, 1997) can involve two approaches: the use of a formal mechanism or intervention that uses punishment to ensure compliance, and the use of incentives to change behaviour to comply with the government's target. The former uses legal control and imposes sanctions on those who break rules. Yet despite the simple logic of the punishment model, a weak judicial system may not prosecute offenders effectively, so that many rule-breakers escape sanction and professionals no longer take the rules seriously. The incentives approach, on the other hand, aims to motivate or encourage changes in behaviour that will enable actors to comply with the intended policy. The use of financial incentives, for example, has been shown to persuade health workers to accept postings to rural areas in South Africa, Kenya and Thailand (Blaauw et al., 2010).

Nevertheless, there seems to be little evidence on the role of incentive-based regulation to improve health worker performance. A study that examines the impact of target payments on doctors working in the British National Health System (NHS) shows an increased number of 
cervical smears taken following the introduction of the scheme, though it was unclear if such response would continue in the long run or perhaps increase doctors' proficiency in the practice (Hughes, 1993). A similar study of the quality and outcomes framework (QOF) in the British NHS, which is an incentive programme meant to reward General Practitioners (GPs) to provide quality primary care to patients, shows that the scheme is cost-effective (Walker et al., 2010). However concerns have been expressed that doctors may concentrate efforts on the incentivised activities while reducing their attention on other activities, which are also beneficial and may have an insignificant effect in tackling health inequality gap in the UK (Thorne, 2016). Elsewhere, evidence from an evaluation of the effects of performance-based incentives programme focusing on HIV and maternal/child in Mozambique shows significant increases in the delivery of prevention of Mother-to-Child HIV and maternal and child services (Rajkotia et al., 2017).

On the other side of the argument, a systematic review study in low-and-middle income countries shows no empirical evidence linking payment to performance (Carr et al., 2011). A study in Afghanistan on performance incentives suggests that low bonuses limited the intended positive effects of the scheme (Alonge, Lin, Igusa, \& Peters, 2017), and in Nigeria, health care providers were sceptical about a successful implementation of payment by incentive scheme due to uncertainty and distrust in a system known for a lack of transparency and corruption (Ogundeji, Jackson, Sheldon, Olubajo, \& Ihebuzor, 2016).

\subsection{Who are the regulators?}

Whilst government may be responsible for identifying the policy objective(s) to be regulated, it is not feasible to implement its policies without the assistance and cooperation of other actors. Other actors that may be involved in health system regulation include, medical professionals, the Ministry of Health, local and international non-governmental organizations, consumer groups, community representatives, the pharmaceutical industry, paramedical workers and others (Ensor \& Weinzierl, 2007). The extent to which other actors work in ways that support the objectives of a regulation may depend on availability of resources and other contextual issues such as the strength of the judicial system and the impact of 'voice' from consumer groups. In doing so, an "institutionalized co-production" process may be created 
where service provision and regulation are both co-produced by the state agencies and organized citizen groups (Bloom, Standing, \& Lloyd, 2008). However, the extent of voice given to organised citizen groups in developing countries as co-producers in service provision and regulation has been undoubtedly low due to over centralisation of authority and a lack of patients' rights (Hongoro \& Kumaranayake, 2000).

Enforcement of regulation is not without a cost to government. Even where regulations exist, enforcement may remain low (Berman \& Cuizon, 2004). The transaction cost of implementing regulation in developing countries is one of the major enforceability challenges (Kumaranayake, 1997). For example, the implementation of the District Health System in Enugu State, Nigeria, was challenged by irregular monitoring and evaluation of staff, and a lack of funding for the programme (Uzochukwu, Onwujekwe, \& Ezumah, 2014), which suggests that poor implementation of regulation might sometimes be an outcome of poor funding.

Apart from the cost involved in enforcing regulation, the capability of government to use command and control as a strategy for regulation has been noted as problematic in LMICs (Ensor \& Weinzierl, 2007). Policy makers and bureaucrats may not have the capability to monitor the nitty-gritty of regulation despite initiating policies. Evidence from developing countries indicates more focus on inputs used in producing health services than on outputs (Ensor \& Weinzierl, 2007; Hongoro \& Kumaranayake, 2000). For example, the cases in Zambia and Tanzania show that regulation has been largely focused on licensing of facilities, registration of practitioners, rather than quality of output that can protect consumers. Despite a lack of capacity, some governments have demonstrated their capabilities to regulate successfully (Bloom, et al., 2008). For instance, the Nigeria National Agency for Food and Drug Administration and Control (NAFDAC) under the late Professor Dora Akuyili, in an attempt to stamp out fake drugs, opened up a new paradigm of trust by using the authority of the state to clamp down on the vested interests of "powerful actors" who import fake drugs into the country.

There exist different regulatory frameworks used to manage private practice of public sector physicians in many health systems. But the extent to which these policies are implemented effectively differs across countries (Berman \& Cuizon, 2004; Jan, et al., 2005). Several 
factors may account for these differences such as effective law and order, availability of human and financial resources, nature of the private health market and stage of health system development.

\subsubsection{Banning/prohibition}

Banning dual practice means that the practice is not permitted officially. Countries that banned DP include Greece (Mossialos, Allin, \& Davaki, 2005) from 1983 - 2002, some states in India (Barman \& Cuizon, 2004), Canada (Flood \& Archibald, 2001), China (Bian, Sun, Jan, Yu, \& Meng, 2003), and Portugal (Oliveira \& Pinto, 2005). The Chinese government has attempted to use reforms to gain control of doctors' professional practice, perhaps, to allow the authority to gain control over doctors' private earnings (Yang, 2006). A 1985 policy allowed Chinese public-sector doctors to render professional services privately on a fee-forservice basis, but they were not authorized to establish private practices as businesses unless they resigned from the public sector (Yang, 2006). This move, however, did not prevent all private practice among doctors, but rather drove it underground. In Japan, public sector doctors are only allowed to do private practice on non-working days (Garcia-Prado \& Gonzalez, 2007). Evidence from these countries shows that compliance with regulations was rarely achieved; instead sharp practices were part of the unanticipated consequences of the ban. For instance, Greece experienced an increase in informal payments for health care following the ban, and most senior doctors moved to the private sector (Mossialos, et al., 2005).

A similar move to the private sector by senior doctors was also witnessed in some states in India (Kiwanuka et al., 2011). In a country where the private sector is lucrative and depends largely on labour supply from the public sector, a ban may have a ripple effect on both private and public health care delivery, as in Nigeria, where the majority of public sector doctors engage in private practice (Akwataghibe, et al., 2013). Similarly, an exodus of skilled public doctors to the private sector was also witnessed in Mumbai due to the ban on DP (GarcíaPrado \& González, 2011). The violation of a ban on DP by doctors may suggest tacit opposition to policies that threaten to control their professional practice. 
Proponents of the ban approach have argued that it can be a good policy if the private sector is not competitive, and if both public and private healthcare sectors are close substitutes (Brekke \& Sørgard, 2007). Basically, both may not be close substitutes due to differences in cost, type and level of hospitals. However, evidence from LMICs suggests that a ban is rarely enforced (Jan, et al., 2005). Moreover, banning DP raises the possibility of a brain drain in the public sector as was the case in Greece and India (Berman \& Cuizon, 2004; Mossialos, et al., 2005), as well as increased use of informal payments to supplement public sector salaries (Ferrinho, et al., 2004b; Jan, et al., 2005). In China, despite the ban (Bian, et al., 2003), DP is still a common practice among public sector doctors.

It is argued that a ban is only viable where government or professional regulation is effective, but certainly not in LMICs (Hipgrave \& Hort, 2013). There seems to be a dearth of literature on any LMIC that combines an efficient health system with a ban on DP. It has also been noted that banning dual practice even if it is enforceable should never be desired (Ferrinho, et al., 2004b). The consequences of banning DP and cost of allowing it is like a double-edged sword. The weakness of health systems in developing countries does not seem to support banning, and possible opposition from the professional body has the potential to further cripple service delivery in the public sector.

In a health system characterized by weak enforcement of regulation, low public-sector salaries, a lucrative private sector, and battered public trust in the public system, banning DP might drive the practice underground. The challenge before any government, particularly those of the LMICs that have the policy option of a ban, is to conduct a policy assessment of the overall consequences for service users, physicians, and the public sector.

It is therefore, in the interest of governments in LMICs to strike a trade-off between a ban and allowing DP with some restrictions to save the public health care system. In Nigeria, banning DP may be difficult to implement and could drive the already established consultants and specialists out of the public sector; this might pose a potential risk to the public sector. 


\subsubsection{Financial restrictions}

There are variations of DP restrictions in many countries. The two main types of restrictions to be discussed here are financial and licensure restrictions. Dual practice may be allowed with restrictions. One type of restriction is financial, although the form varies across countries. These are restrictions on private-sector earnings, incentives offered to physicians to work in the public sector, and salary increases for public-sector doctors.

Countries with restrictions on private-sector earnings include the UK, which allows full-time NHS consultants a $10 \%$ salary top up, while France allows $30 \%$ private earning for public sector physicians (Garcia-Prado \& Gonzalez, 2007; Johannesssen \& Hagen, 2014; Morris et al., 2008). Under the maximum part-time contract in the UK, doctors are permitted to engage in DP without restriction, but they forfeit one eleventh of their NHS salary (Humphrey \& Russell, 2004). Government can also limit the maximum quantity of services to be performed in the private sector as a way of limiting private earnings (García-Prado \& González, 2011).

The extent to which there is absolute compliance with these rules is not clear. It has been shown that the $10 \%$ private practice income threshold for the British NHS was exceeded and that total NHS private income varied by age, specialty and Health Authority, with younger consultants earned higher private income than the older age sub-group (Morris etal., 2008). This suggests that a substantial time is spent in private practice for income top up. However, the study failed to show whether doctors who exceeded the private income top up underperformed in their NHS work, although it might contribute to longer wait times if their contractual hours are reduced due to private work.

The challenge in regulating to set private income earning limits is the honesty of the consultant to declare his private fees and the monitoring instrument available to government to oversee private fee earnings of the consultant, either through private medical insurance or patients who pay out of pocket. Whilst developed countries have well-developed financial systems to monitor doctors' private earnings; it might be challenging in developing country context. Thus, in most developing countries, including Nigeria, a greater majority of public sector doctors are engaged in dual practice, making probably twice or more their public income in private practice (Akwataghibe, et al., 2013). Therefore, fixing an income threshold 
for private earnings could pose a tough compliance challenge due to the often-mentioned poor public salary.

Other forms of financial restriction include incentives and contracts to remain in the public sector. In some countries, government has exclusive contracts that allow physicians to work only in the public sector (Jan et al., 2005; Hipgrave \& Hort, 2013; Gonzalez et al., 2007; Berman \& Cuizon, 2004). For instance, the governments of Portugal, Italy, Spain and Thailand provide exclusive contracts for doctors as a way of incentivizing them to stick to the public sector. In Portugal and Italy as well as Spain, there are four available work regimens part-time, full-time, extended full-time and exclusive public-sector work. The level of incentives for these regimens differs, with higher salaries paid to those that spend more time in the public sector than in the private.

In Spain, there is a fixed monthly income and bonus package for doctors on exclusive fulltime contracts. Likewise in Italy, promotions to higher positions are reserved for physicians on full-time public contracts who neither work in outside private sector facilities nor engage in intramural private practice (Garcia-Prado \& Gonzalez, 2007; Oliveira \& Pinto, 2005). The evidence from the Portuguese health system shows that despite this seemingly attractive incentive only a few doctors opted for it (Oliveira \& Pinto, 2005). Moreover, evidence from these countries shows that the option may be costly for government. While financial incentives could attract doctors, the consequence of differential payment for health care workers may need to be considered. In Thailand, for example, differential treatment of health care providers resulted in resentment among other health care workers (Kiwanuka, et al., 2011), but despite the incentives, private practice is still prevalent. Therefore, the option of financial incentives to keep doctors in the public sector needs a thorough assessment to determine its feasibility, challenges and sustainability.

Salary increase is one form of positive financial incentive. It has been used by several countries as a regulatory tactic to limit DP by providing adequate remuneration to publicsector doctors, since it is noted that the major driving force for DP is income enhancement.

A discrete choice analysis of Norwegian physicians' labour supply seems to indicate that salary increase leads to an increase in hours in whichever sector is offering it (Sæther, 2003). 
However other studies from different countries fail to provide conclusive evidence that salary increase results in improved public-sector productivity (Carr, et al., 2011; Macq, et al., 2001; Yang, 2006). For example, despite the $11 \%$ salary increase for junior doctors and almost $250 \%$ for hospital directors following Greece's NHS Act of 1983, the new contracts did not improve performance as many senior doctors resigned from the NHS for a full-time private practice (Ferrinho, et al., 2004b). Against this, Hanson and Jack (2010) found that higher wages and quality of housing incentives increased doctors' willingness to accept rural postings in Ethiopia, and Johanessen and Hagen (2014) also found that high wages for extended working hours reduced DP in some specialities in public hospitals in Norway. However, depending on the nature of health system, consultants and specialists who have built reputations in the medical profession may not easily respond to salary increase, perhaps due to their potential to gain market advantage in the private sector (Gabric \& Lazic, 2013).

The quest for higher pay by doctors in developing countries seems endless, particularly in a period of austerity when governments are struggling to merely maintain public institutions. Although, salary increase may have a chance to increase productivity in the public sector the implication for public finances may be huge, particularly in developing countries. Overall, salary increase may not be a good option for LMICs including Nigeria because it is neither feasible nor sustainable.

\subsubsection{Licensure restriction}

Licensure means the restriction of private practice to certain categories of doctors. In some countries certain conditions must be met before a physician can undertake private practice. In Kenya and Zambia, for example, only senior doctors are permitted to engage in dual practice (Berman \& Cuizon, 2004; Garcia-Prado \& Gonzalez, 2007). Nevertheless, evidence shows that some of the junior doctors engage in private practice under their senior colleagues' authority and direction (Kiwanuka, et al., 2011). In Indonesia doctors must work in government service for three years before they can provide services in the private sector (Berman \& Cuizon, 2004).

In the Nigerian context, the Nigerian Civil Service Rule allows private practice, but forbids the use of official work hours for private practice. The Rule also allows only consultants and 
senior doctors with more than 10 years post-registration experience to provide care outside the hospital where they work (MDCN, 2008). In reality these rules are not followed, and many consultants on full-time public contracts have well-established private clinics or hospitals where they engage in DP (Osuagu, 2010). Licensure restriction, whatever form it takes can be resisted by the professionals covertly or overtly.

\subsubsection{Intramural Practice}

Intramural practice allows doctors in public hospitals to establish private practice in the public health facilities where they work. The objective is to allow public doctors to use the public facility to carry out their private practice. This policy aims to maximise the availability of doctors in the public workplace. The practice exists in some European countries such as Germany, Ireland and France. In Germany, a public sector doctor can admit a private patient on a fee-for-service basis and then reimburse the hospital from the private fee received (Rickman \& McGuire, 1999).

Available evidence on intramural practice suggests that it has promise as a way of managing DP while avoiding harm to the health system. As indicated by Kiwanuka et al.,(2011), the practice has the potential to limit the damage DP may do to the work of public facilities as it seems to guarantee close monitoring and supervision of doctors. In Italy, there is a mandatory 6-12\% bed space reservation for private patients in public facilities (France \& Donatini, 2005), whereas in Austria, bed space allocation to private patients in government health facilities must not exceed 25\% (Garcia-Prado \& Gonzalez, 2007). In the Irish health system, about $20 \%$ of private bed spaces are allowed in public hospitals (Wiley, 2005). The situation in Portugal, Spain and Peru seems quite the opposite, as there is a ban on the use of public hospitals to treat private patients (Garcia-Prado \& Gonzalez, 2007; Jumpa, et al., 2007).

In Nigeria, intramural practice is permitted in public tertiary hospitals, which allows consultants to bring in their private patients. However, the practice is not fully implemented due to challenges facing the public sector such as a lack of basic facilities. As a result, consultants would prefer to treat their private patients in their private clinics and hospitals away from the government facility. Other challenges include an absence of coherent plans on monitoring and supervision to ensure compliance. Moreover, because consultants receive less 
private income from intramural practice than they would get from extramural work, many are disinclined to give up extramural practice.

Although, this intramural approach shows promise in some developed countries, the application of the policy in developing countries may not achieve positive results due to poor infrastructure and oversight. There may be a compliance challenge with being honest about the official number of hours allotted for private work and the allowable private income threshold. Social discrimination resulting from differences in the quality of private and public care could as well raise issues about unequal access.

\subsubsection{Tolerating dual practice}

There seems to be a permissive attitude to dual practice in some health systems in subSaharan Africa and Asia as a way of allowing private activities to supplement poor public salary (Eggleston \& Bir, 2006). It is reported that in some countries in Africa, salary for health workers is poor to the extent that extra income from activities outside the regular work cannot be ignored (Roenen, et al., 1997). For instance, in 2009, physicians in Uganda earned between $\$ 300$ and $\$ 630$ per month depending on grade level (Ligia, 2014).

Tolerance for DP appears to be greater in countries where it is prevalent. In Bangladesh, over $80 \%$ of public doctors are engaged in DP (Gruen, et al., 2002). There are no medical practice restrictions in Egypt where more than four fifths of private doctors have public sector jobs (Berman \& Cuizon, 2004). In Cape Verde, private sector medicine is liberalized and private practice within the public premises is legally permitted outside regular shifts (Russo et al., 2013). The country's overall economic condition and a lack of capacity on the part of government may have contributed to its inability to initiate policies that could restrict income generating activities of doctors. In Guinea Bissau also, private medical regulation is limited, and private practice inside government facilities is very common and unregulated (Russo et al., 2013). Typically, where private practice is legalized with minimal government intervention, DP may become commonplace.

\subsubsection{Regulating the private sector}

Restricting private sector activities is one of the ways of reducing dual practice and making it less attractive to public providers. In the Canadian system the private sector can only provide 
treatments not classified as medically necessary services in the core benefits package of the public insurance schemes. Services that are accessible in the public system cannot be purchased in the private system (Kiwanuka, et al., 2011). By disallowing private providers access to public funding the financial drive for dual practice is being reduced (Flood \& Archibald, 2001).

Conversely, while the Canadian system tends to reduce the appeal of the private sector for professionals and patients, the Nigerian system allows full-time public-sector consultants with established private hospitals to be accredited as National Health Insurance Service (NHIS) providers with health insurance policy holders (enrolees) assigned to them to deliver a set of services for a fee additional to their public income. While access may be expanded with this model, it appears as a push factor for private practice.

As an alternative, price regulation in the private market has been suggested as a strategy for limiting adverse moral hazard in the public sector and improving consumer welfare (Biglaiser $\&$ Ma, 2007). The authors use economic modelling to show that the physician working under price regulation will not increase private sector income by spending more time in the private sector since the price level is set by a health authority. They believe that a price ceiling in the private sector will limit adverse behaviour. Setting up a price regulation in the private sector may be difficult due to the inherent technicalities involved in deciding a threshold for a treatment cost across specialities, and disease-type. More so, compliance might be difficult, and enforcement may be rarely achieved, particularly for countries with a poor record of implementing regulations. Furthermore, full-time for-profit private practitioners may find it difficult to cope in the market due to the price controls that limit the profitability of private services.

\subsubsection{Self-regulation}

Self-regulation is another regulatory mechanism used in some countries to monitor DP. The use of a professional body as a custodian of medical practice is premised on its ability to influence its members to comply with professional ethics. Self-regulation has been applauded for its strength in discouraging undesirable practices among medical professionals. It is also argued that regulation by peers through an organised reputational mechanism could prove 
effective in improving the practice of medicine (Ensor \& Weinzierl, 2007; Garcia-Prado \& Gonzalez, 2007; Jan, et al., 2005). Despite the fact that "bad apples" in the profession have infiltrated it, self-regulation is still viewed as a good policy option given the nature of medicine (Collier, 2012).

Self-regulation may not be effective for two reasons, either the profession lacks resources to regulate its members or it is unwilling to operate against its own membership (Hongoro \& Kumaranayake, 2000). For example, the Medical Council of Zimbabwe failed to publicize cases of malpractice for fear of exposing its members' incompetence to the public and damaging the profession's reputation. The lack of resources to carry out effective monitoring of members could on the other hand, affect the activities of the group. In Thailand, there were few resources available to the medical profession to monitor members' activities, and despite several complaints received by the Medical Council of Thailand, only about 10-20\% of those cases were given attention (Hongoro \& Kumaranayake, 2000). The fear of delegating selfregulatory responsibility to the medical profession arises from the risk of what Ensor \& Weinzierl (2007) referred to as "regulatory capture", a situation where professional interest overrides that of the public it is meant to protect.

Public disapproval of medical negligence in recent time has raised concerns about selfregulation, and thus, prompted a shift from the traditional medical regulation to greater external influence, for instance, in the UK (Collier, 2012). Today, a substantial number of non-professionals are members of the General Medical Council (GMC) (Gladstone, 2000). The perception of the profession as having high ethical standards meant to protect the public may have been replaced with doubt and mistrust (Humphrey \& Russell, 2004). Others have argued that the use of a collegial system to regulate work in the health sector has not worked due to conflicts of interest (Cruess \& Cruess, 2005). There seem to be continuing doubts about the status of the doctor as a selfless principal interested in the welfare of his agent without some underlying self-interest.

There seems to be no accountability on the part of doctors to patients for negligence, and little likelihood of being forced to pay compensation, particularly in a developing country such as Nigeria. Thus, the principal/agency relationship that forms the basis of doctor/patient 
interaction is compromised as trust is reduced. In Nigeria, for example, there is a lack of published data on errant doctors, which suggests that some erring doctors might have been shielded from scrutiny by the Council. Moreover, channels open for reporting erring professionals are limited whilst consumers' voice is not integrated in the profession's regulatory framework.

It is crucially important to note that variations in dual practice policies reflect health systems' differences and stages of development. Therefore, policies on dual practice are likely to reflect local circumstances and realities. Evidence that could feed into any policy decision on DP should be based on research findings in the country studied. There is no one-cap-fits-all approach.

\subsection{Gaps in knowledge}

Regarding regulation, the main gap explored in the thesis concerns the applicability and relative importance of these different regulatory approaches in the Nigerian context. Drawing on interviews with the various stakeholders the thesis will explore perceptions of how the different mechanisms operate, and views about future reform.

\subsection{The power of the medical profession}

Although this thesis focuses more on the applied literature of dual practice and mid-range theory about how DP works, it is important to say something about how the phenomenon fits with wider social theories about professions and power. These theories were developed mainly from observation of professionals in Western countries, but although their degree of applicability to a developing country like Nigeria needs to be investigated empirically, they seem at face value to have considerable relevance.

\subsection{Findings from the profession's literature}

A key proposition from both the British and American literature is that the medical profession has reached an accommodation with the state which affords it discretion to determine its professional standards and the conditions of medical practise, as well granting certain 
privileges (which may include the right to undertake DP), in return for keeping health services running smoothly. Klein (2006: 144) writes of "the implicit concordat between the State and the medical profession forged by the creation of the NHS, whereby the former accepted the autonomy of the medical profession in decisions about the use of resources while the latter accepted the right of the State to set the budgetary constraints within which it worked." Freidson (1970a: 23-24), (Freidson, 1970b) in his classic study of the US medical profession, writes: "The most strategic and treasured characteristic of a profession - its autonomy - is owed .... to its relationship to the sovereign state from which it is not ultimately autonomous". Freidson $(1970 \mathrm{a} ; 1970 \mathrm{~b})$ has suggested that the state is the foundation on which any analysis of a profession should be based because its source of power and authority comes from the state. The image of the socio-political bargain between the State and profession has at least one critic (see Jacobs, 1988), but has attracted support from many writers (Davies, 2014; Giaimo, 1995; Harrison, Hunter, \& Pollitt, 1990; Salter, 2000). Such an agreement may include a 'regulatory bargain' (Stacey, 2000) which establishes a balance between professional self-regulation and regulation by external organisations, usually government agencies. Salter and Davies both discuss how such bargains may change over time. This suggests that different bargains obtain at different times and in different healthcare systems so that the present arrangements in Nigeria are not static and may be modified further over time.

The medical profession is the main professional body involved in such bargains with the state in the healthcare domain because it exercises control over the areas that fall under the jurisdictions of other healthcare professions (Freidson, 1970a). In most countries the medical profession has over time achieved substantial cultural authority, economic power and political influence (Starr, 1982). This gives it overriding authority over others in the health division of labour (Barnett, Barnett, \& Kearns, 1998). Its position as the "controller" of healthcare organizations and terms of work may have a legal basis in the law that determines licensing requirements and the scope of practice of medicine and cognate professions (Barnett, Barnett, $\&$ Kearns, 1998). The medical profession is licensed as the monopoly provider of such activities as formal diagnosis, the direction of treatments and the prescribing of drugs, which would otherwise be virtually inaccessible to the public (Freidson, 1970b). 
Medical doctors have attained a position where their collective dominance is institutionalised and politically rubber-stamped to the extent that the organisation and outputs of healthcare systems are rarely questioned by either consumers or governments (Barnett, Barnett, \& Kearns, 1998). Moreover, the ability of the profession to use legal and state regulations to maintain its power allows it to defend its monopoly and consequently, achieve an unbalanced relationship with other health professions (Ovretveit, 1985). Thus, according to this author, medicine has been accorded the right to direct and evaluate the work of others but is never subject to the reverse.

Some have argued that professional dominance is gradually weakening due to market reforms, the internal fragmentation of the medical profession following the appearance of new specialties, and the rise of general managers. The medical space, which was managed and controlled by the profession, is, today welcoming non-doctors and other professions who are making strong contributions. Bureaucratization and the increasingly hiring of doctors as salaried employees rather than independent contractors are other factors that are said to be reducing professional dominance (Numerato, Salvatore, \& Fattore, 2012).

Challenges to the profession's power come from various directions. Governments in many countries have responded to global trends in health market reform and system restructuring that has the potential to affect the profession's power. For example, new public management techniques that seek greater accountability via performance target, monitoring and control (Barnett, et al., 1998) and the increased use of rules and incentives intended to change the way doctors perform (Hanson \& Jack, 2010) have had impact in many countries. Similarly, evidence-based medicine and the top-down imposition of clinical protocols and guidelines in public hospitals and (where standardisation is seen to bring cost savings) in the private sector have meant that doctors often perceive that their autonomy is being eroded. In the United States 'managed care' and the rise of Health Maintenance Organizations and Preferred Provider Organisations run to maximise profits illustrate this trend. There is also a rise in consumerism whereby patients are becoming more knowledgeable about their rights and accessing better information about their conditions and treatment options from the internet and elsewhere. All these factors challenge the profession's dominance, but there is evidence 
that it has generally been highly successful in adapting and defending its jurisdictional territory and power. In the UK, Harrison \& Pollitt (1994) show how doctors successfully evaded attempts to control them through the new contracts and disciplinary structures introduced as part of the NHS internal market reforms. Similarly, in the US the profession helped block the Clinton reform proposal to regulate the supply of physicians (Barnett, et al., 1998), and has successfully lobbied to roll back aspects of the more recent Affordable Care Act reforms (Dolgin \& Dieterich, 2012). The varied tactics that doctors use to resist management control are well described in (Numerato, et al., 2012) review of the literature in this area.

The term countervailing power has been utilised by (Light, 1991) and (Harrison \& Pollitt, 1994) (borrowing a phrase from J K Galbraith's 1952 book, American Capitalism) to describe the situation that arises when professionals resist a new management order. These writers argue that one form of professional resistance is for doctors to colonise management level positions and then use that power to resist external influences. The emergence of a management-oriented group of doctors has led to the idea of "re-stratification". Freidson (1994) rejected the idea that medicine was being de-professionalised so that it was losing its monopoly over its area of expertise, and argued that what was instead happening was that an elite stratum of medically-qualified managers was separating itself off from 'rank and file' doctors in clinical practice. According to him, "Professionalism is being reborn in a hierarchical form in which everyday practitioners become subject to the control of professional elites who continue to exercise the considerable technical, administrative, and cultural authority that the professions have had in the past" (Freidson 1994: 9). According to the re-stratification thesis the impact of bureaucratization is reduced because doctors are being directed by fellow doctors. Furthermore, it is argued that the reason some doctors break away to become managers is not to collude to erode the profession's power, but to ensure that it is resisted and halted.

The re-stratification thesis points to one tactic the medical profession employs to retain power in a changing healthcare environment, but it was developed to explain physician behaviour in Western countries and one needs to be cautious in applying it in its entirety to developing 
countries. In the latter it is rarely the case that a new type of hybrid physician manager is displacing a well-established cadre of general managers. Hughes (2014) explains that many countries move through a "kind of natural history whereby early control of health care organizations by physician administrators gave way to an era when professionals were supported by bureaucratic administrators, and then to the appearance of more powerful general managers working in corporatized or 'new public management' (NPM) environments that threaten to erode professional power".

However, Nigeria, like many developing countries, remains at a stage where the Ministry of Health and senior administrative positions in the public healthcare system are almost exclusively staffed by medically-qualified office holders. The idea of an elite group of doctors in senior management and political positions is relevant for Nigeria, but the group has been in charge for some time rather than because doctors have recently seen some advantage in moving into management positions. In fact, developing countries have mostly not followed the same path as developed Western countries where re-stratification has been observed. In countries like Nigeria an older form of administration that was long dominated by medical office holders is still strong, even if now coming under some challenges. Even if the stage is different, the logic of a situation where doctors manage doctors means that doctor power is maintained in much the way Freidson argues would arise as a result of re-stratification.

In Nigeria, the challenge to the traditional order of doctors in charge at all levels, has come from non-physician professionals and managers who have tried to move Nigeria closer to the ideas of interdisciplinary team working and general management that have been influential in developed countries. In recent years the non-physician professions in the health sector have organised a campaign to protest against the alleged "hegemony" of the medical profession (Adeloye, et al., 2017; Alubo \& Hunduh, 2017). They established a formidable alliance named the Joint Health Sector Unions (JOHESU) and incorporating the Nigerian Union of Allied Health Professionals, the Non-Academic Staff Union, the Nigerian Union of Nurses and Midwives, the Senor Staff Association of Hospitals, Research Institutes and Associated Institutions; and Medical and Health Workers Union, with the main objective of synergising their strengths to tackle what they considered to be disproportionate doctor power. The major 
areas of dispute between this alliance and the medical profession include 1) who becomes the Chief Medical Director (CMD) of government hospitals, 2) alleged unfairness regarding differences in emoluments and career progression, appointments within the Ministry of Health, and the appointment of the Chairman of the Medical Advisory Committee (CMAC) (Alubo \& Hunduh, 2017). Doctors claim that the leadership of government hospitals is their legal right and have therefore remained in their positions as the CEOs of government tertiary hospitals. Furthermore, other positions available in running teaching hospitals were all taken by physicians using their dominant power and side-lining the non-physician group. The two prominent administrative positions - CMD and CMAC - are occupied by doctors, and recently a third one has been created - Deputy Chairman of the Medical Advisory Committee (DCMAC) in tertiary hospitals - that has also been taken by a doctor appointee (Alubo \& Hunduh, 2017). Various efforts made by the non-physician group to open these positions to non-doctors were unsuccessful. During this period the doctors pushed to get a rule made stating that non-physicians cannot be appointed as directors to the boards of tertiary hospitals, so as to avoid opposition in the management meetings. There is a growing sentiment among doctors that they must assert their responsibility for directing other members of the health team to ensure effective patient care (Ojo \& Akinwumi, 2015), even when the public perception is that most doctors lack managerial competency.

On the issue of salary and emolument, doctors were able to achieve a separate salary structure for their members with a salary gap between physicians and non-physician groups (Alubo \& Hunduh, 2017). The physician group has always used industrial action to force the state into negotiation, which often ends up favouring their demands. Efforts made by the non-physician group to establish their presence at senior administrative management meetings in government hospitals were thwarted by a coalition of physicians, senior hospital managers with medical backgrounds, the medically-dominated government executive council and the legislature. Thus, the organisational power and political influence of the Nigerian medical profession remains highly visible. 


\subsection{Gaps in knowledge}

The theory of professional dominance appears relevant in this study, but empirical material on its applicability to the Nigerian context is thin. We shall see from the data chapters that the medical profession remains very strong in Nigeria. This suggests that an attempt to increase compliance with the rules regulating dual practice in Nigeria might well be constrained by doctors using their cultural authority and professional power. Given the fact that doctors are well represented in government policy making circles and senior management it is highly likely that they may seek to limit sanctions applying to physician rule-breakers. We will return to these issues in the discussion and conclusion chapters. 


\section{Does dual practice bring benefits?}

\subsection{Benefits of dual practice in the health sector}

This chapter details the benefits of dual practice to the health sector. The socio-demographic characteristics of the different categories of respondent are shown. It shows also how service users perceive the benefits of dual practice based on their hospital visits experiences. It narrates the perspectives of policy makers and other stakeholders on the benefits of dual practice. The views of doctors working in the public sector and how they perceive the benefits to service users, the health sector and the DP practitioner are described. 


\subsection{Physicians' and stakeholders' socio-demographic Characteristics}

Table 1: Socio-demographic characteristics of dual practitioners

\begin{tabular}{|c|c|c|c|c|}
\hline Variables & $\begin{array}{l}\text { Consultants } \\
(\mathrm{N}=8)\end{array}$ & $\begin{array}{l}\text { Snr Registrar } \\
(\mathrm{N}=3)\end{array}$ & $\begin{array}{l}\text { Registrar } \\
(\mathrm{N}=1)\end{array}$ & $\begin{array}{l}\text { General Practice } \\
(\mathrm{N}=3)\end{array}$ \\
\hline \multicolumn{5}{|l|}{ Sex } \\
\hline Male & 8 & 3 & 1 & 3 \\
\hline Female & 0 & 0 & 0 & 0 \\
\hline \multicolumn{5}{|l|}{ Highest Ed level } \\
\hline PART1 Fellowship & - & 3 & - & - \\
\hline *FWACP & 8 & - & - & - \\
\hline MBBS & - & - & 1 & 2 \\
\hline MSc Pub health & - & - & - & 1 \\
\hline \multicolumn{5}{|l|}{ Speciality } \\
\hline Paediatrics & 4 & - & 1 & - \\
\hline Obstetrics \& Gynae & 2 & 2 & - & - \\
\hline Orthopaedic & 1 & 1 & - & - \\
\hline General Medicine & - & - & - & 3 \\
\hline Cardiology & 1 & - & - & - \\
\hline $\begin{array}{l}\text { Mean duration in } \\
\text { service(yrs.) }\end{array}$ & 8 & 6 & 7 & 17 \\
\hline
\end{tabular}

*Fellowship of the West African College of Physicians

The DP group comprises eight medical consultants, three senior registrars, one registrar and three general practitioners. Five of the respondents specialised in paediatrics, four in obstetrics and gynaecology (Obs and Gynae), one in cardiology, and three in general medicine, while two were in orthopaedic/trauma medicine. The mean duration in years of service for the consultants is eight years, the senior registrars, six years and the registrars, seven years. The general practice doctors have spent 17 years in service on average. The respondents were all male doctors. 


\subsection{Socio-demographic characteristics of non-dual practice}

\section{physicians}

Table 2: Socio-demographic characteristics of non-dual practitioners

\begin{tabular}{lllll}
\hline Variables & $\begin{array}{l}\text { Consultants } \\
(\mathbf{N}=7)\end{array}$ & $\begin{array}{l}\text { Snr Registrar } \\
(\mathbf{N = 4})\end{array}$ & $\begin{array}{l}\text { Registrar } \\
(\mathbf{N = 2})\end{array}$ & $\begin{array}{l}\text { General Practice } \\
(\mathbf{N = 2})\end{array}$ \\
\hline Sex & 3 & 4 & 1 & 0 \\
Male & 4 & 0 & 1 & 2 \\
Female & & 4 & - & - \\
Highest Ed level & - & - & - & - \\
PART1 Fellowship & 7 & - & 2 & 1 \\
*FWACP & - & - & - & 1 \\
MBBS & - & & & - \\
MSc Pub health & & - & - & - \\
Speciality & 4 & - & - & - \\
Paediatrics & - & - & - & 2 \\
Obstetrics \& Gynae & 2 & - & - & - \\
Orthopaedic & - & 1 & 2 & - \\
General Medicine & 1 & 3 & - & 2 \\
Community Medicine & - & 3 & & \\
Dentistry & 10 & & & \\
Mean duration in & & & & \\
service (yrs.) & & & & \\
\hline
\end{tabular}

*Fellowship of the West African College of Physicians

The non-DP group consisted of seven consultants, four senior registrars, two registrars, and two general practitioners who were on full-time contracts in the public sector (Table 2). Their specialties were dentistry, orthopaedics, paediatrics, community medicine and general medicine. The mean years in medical practice for the consultants was 10 years, three years for the senior registrars, five years for the registrars and two years for the general practitioners. They comprised eight females and seven male public doctors who worked exclusively in the public sector at the time of interview. 


\subsection{Socio-demographic characteristics of policymakers/stakeholders}

Table 3: Socio-demographic characteristics of policymakers/stakeholders

\begin{tabular}{|c|c|c|c|c|}
\hline *Names & Occ. & $\begin{array}{l}\text { Highest } \\
\text { Edu level }\end{array}$ & Position & Duration \\
\hline Dr Oloto & $\begin{array}{l}\text { Medical } \\
\text { practitioner }\end{array}$ & FWACP & $\begin{array}{l}\text { Chairman } \\
\text { SHB }\end{array}$ & 4 years \\
\hline Dr Buo & $\begin{array}{l}\text { Medical } \\
\text { practitioner }\end{array}$ & $\begin{array}{l}\text { MBBS } \\
\text { (PT1) }\end{array}$ & NMA SEC & 3 years \\
\hline Dr Atu & Civil Servant & MBBS & $\begin{array}{l}\text { Dir. MEd } \\
\text { Services }\end{array}$ & 9 years \\
\hline Dr Iyo & $\begin{array}{l}\text { Medical } \\
\text { Administrator }\end{array}$ & MBBS & $\begin{array}{l}\text { Asst. } \\
\text { Director } \\
(\mathrm{MDCN})\end{array}$ & 9 years \\
\hline Dr Huso & Civil Servant & $\mathrm{PhD}(\mathrm{PH})$ & $\begin{array}{l}\text { Permanent } \\
\text { Sec. }\end{array}$ & 8 years \\
\hline Dr. KO & $\begin{array}{l}\text { Medical } \\
\text { Practitioner }\end{array}$ & FWACP & $\begin{array}{l}\text { NMA } \\
\text { Chairman }\end{array}$ & 3 years \\
\hline Mr. Eki & Civil Servant & MSc. & Sec. SHB & 4 years \\
\hline Dr. Phiri & $\begin{array}{l}\text { Public } \\
\text { Servant }\end{array}$ & MSc & $\begin{array}{l}\text { Chairman, } \\
\text { House Cttee } \\
\text { on Health }\end{array}$ & 6 years \\
\hline
\end{tabular}

*Names - pseudonyms

In Table 3, key informants interviewed were drawn from the State Health Board (2), the Nigerian Medical Association (2), the Office of the Director of Medical Services of the State Ministry of Health (1), the Medical and Dental Council of Nigeria (1), the Office of the permanent Secretary in the State Ministry of Health (1), and the Office of the Chairman, House Committee on Health of the State House of Assembly (1).

\subsection{Socio-demographic characteristics of FGD participants}

\subsubsection{Socio-demographic characteristics of FGD female group1 participants}


Table 4: Socio-demographic characteristics of participants (1)

\begin{tabular}{llllll}
\hline S/N & Names & Occupation & Edu Qual & Age & $\begin{array}{l}\text { Marital } \\
\text { status }\end{array}$ \\
\hline 1. & Mrs Onyema & Civil servant & NCE* & 26 & Married \\
2. & Mrs Eme & Hair dresser & SSC** & 23 & Married \\
3. & Mrs Chuk & Business & SSC & 27 & Married \\
4. & Mrs Lilian & Business & SSC & 22 & Married \\
5. & Mrs Onyi & Civil servant & BSc. & 28 & Married \\
6. & Mrs Kachi & Corps member & BSc. & 24 & Married \\
7. & Mrs Okwu & Civil servant & BSc. & 38 & Married \\
\hline
\end{tabular}

Names- pseudonyms * National Cert of Education ** Senior School Certificate

Table 4 shows that all the participants were married with an average mean age of 27. Only three of them had a Bachelor of Science degree while three had completed secondary school education with only one person having the National Certificate of Education. Two participants were engaged in business, one a hair dresser, one a corpse member, and the rest were civil servants.

\subsubsection{Socio-demographic characteristics of FGD female group 2 participants}

Table 5: Socio-demographic characteristics of participants (2)

\begin{tabular}{llllll}
\hline S/N & Names & Occupation & $\begin{array}{l}\text { Marital } \\
\text { status }\end{array}$ & Edu Qual & Age \\
\hline 1 & Chi & Teacher & Married & HND & 28 \\
2 & Mal & Civil servant & Married & HND* & 28 \\
3 & Chioma & Trader & Married & OND** & 25 \\
4 & Uka & Self-employed & Married & SSCE & 27 \\
5 & Favour & Self-employed & Married & HND & 34 \\
6 & Ebere & Trader & Married & SSCE & 40 \\
7 & Ruth & Trader & Married & SSCE & 33 \\
\hline
\end{tabular}

Names - pseudonyms *Higher National Diploma ** Ordinary National Diploma

In Table 5, all participants were married. They had a mixed occupational background with three of them trading, one a teacher, two self-employed and one a civil servant. They had a 
mean average age of 31 years. Three of them had a Higher National Diploma while one had OND. The rest had Senior School Certificate.

\subsubsection{Socio-demographic characteristics of FGD male group 3 participants}

Table 6: Socio-demographic characteristics of participants (3)

\begin{tabular}{llllll}
\hline S/N & *Names & Occupation & $\begin{array}{l}\text { Marital } \\
\text { Status }\end{array}$ & Edu Qual. & Age \\
\hline 1. & Praise & Teacher & Single & BSc & 26 \\
2. & Emmanuel & Unemployed & Single & BSc & 26 \\
3. & Ikenna & Unemployed & Single & BSc & 25 \\
4. & Patrick & Applicant & Married & BSc & 29 \\
5. & Michael & Applicant & Married & HND & 30 \\
6. & Okafor & Unemployed & Single & BSc & 24 \\
7. & Onu & Teacher & Married & MSc & 30 \\
8. & Joseph & Unemployed & Single & BSc & 24 \\
\hline
\end{tabular}

*Names - pseudonyms

Table 6 shows that over half of the participants were unemployed, while the majority of them were single and had Bachelor of Science degree. The group had a mean age of 27 years.

\subsubsection{Socio-demographic characteristic of FGD male group 4 participant}

Table7: Socio-demographic characteristics of participants (4) 


\begin{tabular}{llllll}
\hline S/N & *Names & Occupation & $\begin{array}{l}\text { Marital } \\
\text { Status }\end{array}$ & Edu Qual & Age \\
\hline 1. & Emma & Business & Married & BSc & 41 \\
2. & Sab & Driver & Married & SSC & 46 \\
3. & Edu & Business & Married & SSC & 40 \\
4. & Dichi & Business & Married & SSC & 35 \\
\hline
\end{tabular}

*Names - pseudonyms

Table 7 shows that majority of the participants were engaged in business and had secondary school education except one with a Bachelor of Science degree. This group had a mean age of 41 years.

\subsection{Descriptive Likert analysis of benefits of DP}

The author considered using exploratory factor analysis to examine the Likert-type items in the household survey, but in light of the topics addressed and the scale of the study it was decided that a simple descriptive Likert analysis of the survey data would be adequate. Consequently, arithmetic means (and 95\% confidence intervals) are shown in all the tables that follow and are used as a simple way of presenting the strength of agreement or disagreement across the five-point Likert scale to allow comparison of the weight of agreement/disagreement between different factors in a table. As is usual a numerical value was attached to each of the five items (1=Strongly Disagree, 2=Disagree, $3=\mathrm{DK}, 4=$ Agree, $5=$ Strongly Agree), and an average was calculated. Thus, a mean value below 3 indicates that the weight of opinion disagrees with a statement, while one above 3 indicates the weight of opinion agrees, with agreement becoming stronger as the figure rises. It should be noted that there are differences of opinion in the literature regarding the validity of using the mean to analyse Likert data. Critics believe that the transformation of categories such as 'strongly agree' and 'agree' into ordinal data by attaching numbers to them, does not thereby establish that the intervals between the observed values are equal and constant. They argue that use of the arithmetic mean assumes interval data, and that it is more appropriate to use the mode as the average measure with ordinal Likert data. Typically, however, in real world analysis the mean and mode produce very similar results, so that the mean is used here. Readers who prefer the mode can readily calculate this from the number counts in the Tables below. In the 
present thesis relative weight is only of interest when comparison of the importance of different factors is relevant, such as in the question of which factors respondents believe to be most important in motivating doctors to engage in DP. Even here it may be more important to identify a cluster of relevant factors rather than to place them in precise rank order, so the author feels that a simple descriptive analysis is adequate.

In order to facilitate a clear presentation of results the discussion after each table will generally differentiate only between agreement or disagreement (for example, aggregating the 'strongly agree' and 'agree categories') to give a picture of how respondents as a whole viewed particular questionnaire statements. However, there will be examples where the relative numbers of respondents opting for 'strongly agree' as opposed to 'agree' do affect the overall weight of opinion, and the analysis will use the mean data to examine this if it appears important.

Table 8: Benefits of dual practice to service users $(N=407)$

\begin{tabular}{|c|c|c|c|c|c|c|c|}
\hline Question & $\begin{array}{l}\text { Strongly } \\
\text { disagree } \\
\text { n(\%) }\end{array}$ & $\begin{array}{l}\text { Disagree } \\
\mathbf{n}(\%)\end{array}$ & $\begin{array}{l}\text { Don't } \\
\text { know } \\
\text { n(\%) }\end{array}$ & $\begin{array}{l}\text { Agree } \\
\text { n(\%) }\end{array}$ & $\begin{array}{l}\text { Strongly } \\
\text { agree } \\
\text { n(\%) }\end{array}$ & $\begin{array}{l}\text { Mean } \\
\text { [SD] }\end{array}$ & $\begin{array}{l}95 \% \mathrm{CI} \\
\text { (lower- } \\
\text { upper) }\end{array}$ \\
\hline
\end{tabular}


32a). Government doctors give speedier attention to patients in their private practice than in government hospital

32b). Access to government doctors in government hospital is quicker than in their private practice

32c). Government doctors engaged in private practice have more functioning medical equipment than those in government hospitals

$32 \mathrm{~d})$. There is a better quality of care when treated by a government doctor in a government hospital than in his private practice

32e). Government doctors engaged in private practice use their private practice to save lives during strikes in government hospitals

32f) There is quicker access to a specialist in his private practice than in his government hospital

$32 \mathrm{~g}$ ). Instalment payments are allowed more in a private practice than in government hospital

$32 \mathrm{~h})$. There is a shorter wait time to see a government doctor in a government hospital than in his own private practice

32i). There is less bureaucracy (protocol) in private practice than in government hospitals

32j). Patient/ doctor relationship is stronger in a government hospital than in a private practice

32k). Government doctors are less sympathetic to patients in government hospital than they are in their own private practice

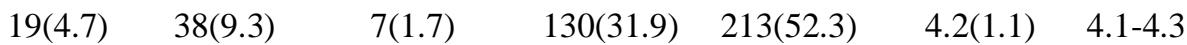

$\begin{array}{lllllll}102(25.1) & 196(48.2) & 16(3.9) & 59(14.5) & 34(8.4) & 2.3(1.2) & 2.2-2.5\end{array}$

$\begin{array}{lllllll}112(27.5) & 147(36.1) & 15(3.7) & 87(21.4) & 46(11.3) & 2.5(1.4) & 2.4-2.7\end{array}$

$\begin{array}{lllllll}68(16.7) & 187(45.9) & 18(4.4) & 87(21.4) & 47(11.5) & 2.6(1.3) & 2.5-2.8\end{array}$

$\begin{array}{llllll}3(0.7) & 5(1.2) & 3(0.7) & 152(37.4) & 243(59.9) & 4.5(0.7)\end{array}$

$\begin{array}{llllll}31(7.6) & 32(7.9) & 6(1.5) & 181(44.5) & 157(38.6) & 4.0(1.2)\end{array} \quad 3.9-4.1$

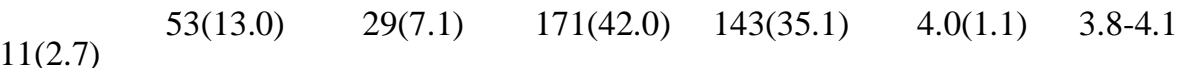

$\begin{array}{lllllll}129(31.7) & 202(49.6) & 11(2.7) & 44(10.8) & 21(5.2) & 2.1(1.1) & 2.0-2.2\end{array}$

$\begin{array}{llllll}8(2.0) & 9(2.2) & 1(0.2) & 175(43.1) & 213(52.5) & 4.4(0.8)\end{array}$

$\begin{array}{lllllll}114(28.1) & 203(50.1) & 29(7.2) & 36(8.9) & 23(5.7) & 2.1(1.1) & 2.0-2.2\end{array}$

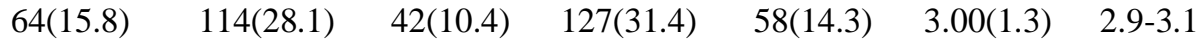

Scores ranged between 1 and 5 
If we examine the importance attached to various possible benefits of DP in Table 8 we can see that time waiting for access to care is a key factor for service users. When for simplicity we combine 'agree' and 'strongly agree', we find that $84.2 \%$ of respondents believed that doctors give speedier attention in their private practice than in government hospitals (Item $32 \mathrm{a})$. When it came to quicker access to a specialist, a near identical number $(83.1 \%)$ believed that private practice offered an advantage (Item 32f). If the question form was reversed then only $22.9 \%$ thought access to doctors was quicker in government hospitals than private practice (Item 32b), and if the question was phrased in terms of wait time only $16 \%$ thought this was shorter in the public hospital (Item 32h). These perceptions of slower access in the public sector may be related to the notion that there is more bureaucracy in public hospitals than private facilities, and indeed $95.6 \%$ of respondents thought this was the case (Item 32i). There was a majority perception that care quality was better in private facilities with $62.6 \%$ disagreeing with the statement that quality was better in the public hospital (Item 32d). However, on the related issue of whether private facilities possessed better equipment only $32.7 \%$ thought that they did (Item 32c). The perception that private practice promotes better patient/doctor relationship may be another factor that makes them attractive. Only $14.6 \%$ of service users thought government hospitals were better in this respect (Item 32j). Surprisingly, service users were more evenly split about whether doctors were more sympathetic to patients in their private practice compared with the public hospital, with $45.7 \%$ agreeing and $44 \%$ disagreeing (Item 32k). Another factor that appears significant is that a large majority of service users $(77.1 \%)$ believe that instalment payments are more readily available in private practice than in public hospitals, something that may lower a barrier to access given that public hospitals will also make a charge.

On the issue of whether DP might be a life saver during a public-sector strike, an overwhelming majority (97.3\%) of service users agreed that this was the case, something that may reflect widespread public unease regarding past waves of industrial action in the public healthcare service.

It appears that patients benefit more from DP as a life saver during strikes in government hospitals. Government hospitals are usually shut down during strikes leaving the private sector as an alternative for those who can afford the cost. Again, bureaucracy seems to deter 
patients in government hospitals, resulting in a preference for private care. The consensus among respondents was that speedier attention and quicker access to specialists in private practice are the main benefits gained by those who use the private sector.

\subsection{Perspectives of respondents on benefits of dual practice in the health sector}

This section deals with findings from different categories of respondents in the study on benefits of dual practice. The responses from service users, policymakers/stakeholders and doctors are presented below.

\subsection{Service users' perspectives}

Service users were the FGD participants who had visited both public and private health facilities and were able to narrate their hospital visits experiences in both sectors. The three benefits of dual practice that emerged from the findings were easier payment plans for private patients, an enhanced doctor/patient relationship, and availability of dual practitioners in their private practice. Each of these benefits is discussed below.

\subsubsection{Easier payment plans for private patients}

In some instances, patients could be allowed a deferred payment, i.e. giving them treatment and allowing payment later. A deferred payment helps those who may not have cash at hand to obtain healthcare services at the time of need. This may not be permissible in the public system. There were instances according to participants when patients could be treated on credit and expected to pay later. In some cases, bills were reduced by DPs as compensation and to attract future patronage.

So, after the treatment and was given my bill, I then asked him [doctor] that since you referred me to this place [doctor's practice] and I came that I deserved a discount. He then told the nurse to give me a discount from the bill he gave me and also gave me very good drugs (FGD 3. P3).

I paid him $N 7000$ (\$23) but he charges N12000 (\$39). Then because of my condition he collected that amount from me. The person that directed me told me 
that another person she referred there also paid N12000 for the same treatment. But if it were in the government hospital, you must pay the total bill, and they will not even have time to take good care of you "(FGD 4, P.5)

...take for instance, I may go to the hospital for one healthcare need or the other and may not have enough money to pay for my hospital bills. In private practice I may receive all the necessary treatments on credit and would be permitted to pay later. This is not permissible in the government hospital (FGD 4, P.4).

In contrast, two of the participants did not agree that a deferred payment is always available in private hospital. They said that from their experience healthcare costs can also be sometimes paid in instalments in the public sector.

Why I said no is that public hospital is always affordable. But in private practice I do not think it is always true that you can pay in instalments for your bills (FGD 2. P5).

The public hospital is always affordable, and their mode of payment can be in instalments. It is not a must that you pay first before treatment is commenced. But if you go to a private practice you must deposit a certain amount of money, probably one third of the cost before treatment can commence. Whether you are about to die or not, what they know is that certain amount must be deposited before you can be treated (FGD 2, P.2).

In a health system that does not cover most of its population in the national health insurance scheme, it may be difficult for many to access healthcare services at the time of need when there is no cash. Instalment payment plan would provide succour for private patients. However, it may not benefit a first-time patient, but older ones who may have built trust and are well known to the facility. So, older patients may have their bills reduced, or be allowed to pay in instalments based on their relationship to the facility. In the public system, bureaucratic rules may not allow those payment systems irrespective of number of visits to the hospital. Though, there may be exemptions for indigent and poor patients in the public system; access to those means-tested benefits may be hard to access. Therefore, easier payment plan tailored to the need of patients could attract future customers and ensure a steady patronage in the private sector. 


\subsubsection{An enhanced doctor/patient relationship}

Doctor/patient relationship seems to be the core and central part of medical practice. The findings show that private medicine as described by Strong (1979) uses the private 'format' with different styles of selling with consultation more personalized and less bureaucratic, which aided interaction with the doctor. This seems to be a sharp contrast with the public sector. They felt welcomed, and the doctors also spent more time to discuss their health conditions in private practice. They had the impression that dual practitioners are more sympathetic to their private patients than they are to public patients in public hospitals.

Those government doctors in dual practice will welcome you very well in their private practice. The moment you come to see them in their private hospitals, they will start treating you without wasting time (FGD 3. P.3)

In private practice, doctors take time to explain things to patients but in government hospitals when they remembered that their salaries have been owed for one or two months; they consider it a waste of time to spend quality time with a patient. So, they might treat you anyhow. In the private sector you are at rest because they will take time to explain things to you (FGD 4. P.7)

Patients want doctors who can make them feel at home. When doctors spend time with private patients, it gives them the opportunity to narrate their illness episodes, and thus, feel cared for. A lack of a warm welcome to a patient could make them hide their feelings. This relationship is rarely established in the public sector as no doctor owns a patient in the public system like private practice. Therefore, showing sympathy for patients is one way of gaining their hearts, and since these characteristics can often influence patients' choice of healthcare provider; those in private practice could use it to gain the attention of more service users. The amount of care and concern dual practitioners show in private practice is apparently business-like. The underlying fact remains that a doctor's income in the private practice depends on their ability to manage their clinics and to attract patients. The attitude exhibited in the public sector does not determine a doctor's promotion or extra income; neither does the number of patients they see daily determine the size of their income. However, this is crucial in private practice if the DP doctor is to break even or make a profit. 


\subsubsection{Availability of dual practitioners in private practice}

Participants agreed that doctors are more readily available to patients for face-to-face contact in private practice than in public hospitals. They agreed that private doctors are more accessible in their private clinics than in the public system.

...I was once rushed to the public hospital and the resident doctor was not there and I was taken to a private hospital, and within ten minutes I was attended to and treated ... (FGD2, P.2)

One of the gains of dual practice is that those dual practitioners do give quicker attention to my healthcare needs in their private practice than in government hospital. Whenever I call on them they answer to my needs (FGD 3. P1).

Again, irrespective of the time you come to the hospital even in the midnight they will pay attention (FGD 4, P.3).

The availability of private doctors in their private clinics seems to be the driving force for patronage. Patients avoid long waiting time, the type found in the public sector. During emergencies, the public sector may be unresponsive to urgent healthcare needs due to absenteeism and delays. The patient's second-best alternative could be the private sector. Dual practice, therefore, provides a safety net for patients in terms of urgent healthcare needs. Additionally, the kind of attention they receive from private practice could be likened to how business owners treat their customers in the real world. Importantly, the availability of dual physicians at the time of need gives service users the confidence that they are always there whenever needed.

\subsection{Doctors' perspectives on benefits of DP}

Respondents mentioned many benefits that DP brought for doctors, patients and the public system.

\subsubsection{Benefits to DP physicians}

Among the benefits that respondents claimed DP brought for doctors were extra income (regarded by many as the primary benefit), professional development, and better work satisfaction, usually connected with increased autonomy in the private sector. 
Many mentioned the importance of DP as a source of extra income needed to augment a poor public-sector salary.

The obvious benefit is that the physician or health practitioner goes home with something at the end of the day. If you like, call it an enhanced take home pay. He gets something from the public service where he is working and also from the private work which he does and that helps to improve his monthly take home pay to take care of his food and other needs. That is the obvious advantage of that practice. (Interview with a DP Consultant)

The financial base of a doctor in Nigeria is very poor compared to other countries, very poor indeed. And you cannot go on like that, even with dual practice you still find yourself below the minimum level that you would expect in other countries. In Nigeria, since there is no thriving health insurance and we are under the ministries as civil servants, the pay is nothing to write home about (Interview with a DP Consultant)

The drive for an extra income seems to be one of the major reasons for private practice, and this is generally framed against the background of poor government salaries rather than a desire for wealth per se.

It was overwhelmingly believed that dual practice can enhance doctors' professional development. Two areas of professional development mentioned were providing training opportunities and increasing doctor's skills. Respondents claimed that private practice brings them into contact with a wider variety of conditions and give better learning opportunities.

It is a very important reason. I remember that, as a house officer, I had the opportunity to look for a job in a private hospital. There I had the opportunity to watch first-hand how a Caesarean Section is done. It also gave me the opportunity to see first-hand how evacuation is done - that is in doing it under supervision. (Interview with a DP Snr. Registrar)

Sometimes, there are just a few cases and you cannot even use these to teach medical students in the public sector. In that situation, you can even invite your resident doctors and tell them to come over to a private hospital where you are practising for them to witness a case and they can learn from that. It gives the doctor, the opportunity to teach the junior doctors and, in the process, they learn (IDI with a DP Consultant)

However, this was an area where respondents gave contradictory accounts. Against the claims that private facilities had a more varied case mix, other informants maintained that only minor 
illnesses are handled in private practice, with the serious health problems most often going to the public sector. Views on the effectiveness of the training in the private sector also diverged.

...patients who go to the public sector tend to be those you cannot manage in the private sector. How many bed spaces do DPs have in their private practices? In training doctors, you need patients with complicated problems, patients with organ failures, so that you can admit them, allow them stay for in the hospital a long time and then you monitor how they recover or eventually die. In the private sector, what you see are common illnesses; most of those with more serious problems in Nigeria always come to the public sector and that may be because they think the cost will be less. It is very difficult to train resident doctors in a private setting, unless it is a well-organised huge multiple set up that involves the four major departments - medicine, surgery, paediatrics and Obs and Gynae with a lot of doctors and good administrators. I do not believe it is good to train junior doctors in the private sector (IDI with a non-DP Snr. Registrar)

I would not totally agree with that, the reason why a residency programme is set up is for you to have that apprenticeship. In a regulated environment, where there is apprenticeship, you can be certain that there is a scheme or a planned programme for what you teach. Apprenticeship in the private sector means that you are assisting regularly in a surgery and, after you've watched me for a while, I will teach you how to do a suture. It is different from a planned programme that is recognised. There is no one to test the knowledge, someone does not have to sit with a person and say 'ok, you are very good'. If you are very good, then come and subject yourself to exams that postgraduate bodies organise to determine if you have really acquired what it takes to make you competent (IDI with a non-DP Consultant)

It is unclear whether the contrasting views in these issues represent partisan positions for and against DP, or whether these accounts also reflect the enormous variation in the size and resourcing of private facilities. In areas where patient flow through public hospitals are low and sizeable private facilities prove more attractive, the claims about enhanced training opportunities may well have validity, but the picture may be reversed elsewhere. Formal resident training, for example, may not be possible in the private sector as the trainee cannot be subject to a formal examination.

While in the public sector there may be a large team of residents handling a single case with a consultant, in the private sector it may just be the consultant with a couple of resident doctors dealing with a case. 
If you are a junior doctor working with a senior doctor, then you have one-on-one on the spot training; unlike in a public setting where you may have about ten residents with a consultant handling a case; you can imagine the number of such cases that are needed for the residents to get hands-on in order to learn. But, when you are working with a consultant [in private practice], a case can only be handled by just you and the consultant. You know that there is more likelihood to get hands-on experience and learn about more cases compared with similar cases in a public hospital with many residents, all struggling to see what the consultant is doing. So, you cannot compare one-on-one teaching with a teacher and so many students. That is why I said that I agree with the learning curve in terms of being hands-on [more with one-on-one than one to many residents]. (IDI with a DP Snr. Registrar)

It does, dual practice can increase one's professional development. What happens is that your patient is the laboratory and your skill is based on your experience. The more you see, the more experience, and the less you see, the less experience (IDI with a non-DP Consultant)

People get better at what they do when they do it regularly. Medicine requires constant practice and seeing many different cases could be an advantage for a doctor.

Some of the respondents suggested that there is job satisfaction in dual practice. This is because they are able to access superior equipment that enhances their performance in the private sector, unlike in the public system that lacks equipment and other basic materials. There is also a sense of satisfaction when they interact with private patients that have come to consult them based on their expertise.

The second one is the satisfaction in practice. The reason I say satisfaction is that when you come here and give them a list of instruments you need to work with, they will not provide them. So, for me to have a full satisfaction in what I do, I can only get that in the private sector. I went for ultrasound training and since I've been here there is no ultrasound to practise what I was trained on. It is only when I go to the private hospital where I also work that I can use an ultrasound scanner for a trans-vaginal scan. So, when I am scanning, I will be seeing what I would want to see. This is not just sending my patient to a private laboratory, but, with the scanner, I can do it there and get my result. That gives me a lot of satisfaction. They are paying well, but that satisfaction I derive is a motivating factor (Interview with a DP Consultant)

... it affords the dual physician the opportunity for a personal interaction with those that really want to see him in the private sector, those that are interested in seeing him due to his expertise. So, there is fulfilment in such personal interaction. (Interview with a non-DP Consultant) 
The two important sources of satisfaction mentioned by respondents for working in the private sector were the satisfaction derived from working with better equipment and the interaction with private patients who have come to seek consultant's expertise.

\subsubsection{Benefits to the public system}

Two benefits of dual practice to the public system were mentioned by respondents. These were to generate revenue for government and the reduction of pressure on public hospitals. However, some of the respondents did not see dual practice to be of any benefit to the public healthcare sector.

Revenue accrues to government by clinic registration and licensing fees. These fees are paid annually for clinic registration and renewal.

Yes, I pay tax. In fact, they charge a lot. They have different kind of taxes. You have to pay and, if you do not pay, you will be in their bad books. So, I pay taxes every year, different kinds of taxes (Interview with a DP Consultant)

They do, if you want to avoid trouble you have to register it and every year you renew your licence to continue to operate your clinic. So, government gets revenue from registering the place and licensing it. This you do every year. You get your private health facility registered, usually with the Ministry of Health. Every year you must update the registration. But you have to obtain the licence to practise from the Medical and Dental Council of Nigeria. That is what you use to practise, both in government and private facilities. (Interview with a DP Consultant)

The taxes paid to government for the licensing and clinic registration are sources of revenue accrued to government. The licensing and clinic registration are renewable every year.

The public system, according to respondents, is under pressure and over-utilised, but private practice helps to reduce such pressure.

You know government hospitals are already not doing well. But at least it reduces the load on public health facilities. Sometimes, you find out that the public health facility is ripping off the patients without giving the services and there is nobody to complain to. Who do you blame? Is it the doctor? You may not even know the doctor because there are so many of them and he may not know you. Is it the facility? (Interview with a DP Consultant) 
Some public hospitals are quite busy and, as such, they may not be able to take the whole patient load; remember what I said before, about high patient load, that bed spaces are very limited, and bed occupancy is also not on the positive side. You find out that, when the load is shared, the facilities in the public hospitals are not overstretched, they are not overused, and hygiene is maintained, the workers have a good morale, they are not overworked, and government is able to match up with demands. (Interview with a DP Consultant)

Private practice seems to reduce the pressure on the public sector by providing alternatives via private clinics and hospitals. Some public hospitals, especially at the tertiary level, can be overloaded with patients. The proliferation of private clinics and hospitals in town by government doctors may, to a large extent, reduce the number of patients accessing public healthcare. In a way, the public system benefits by not being overstressed and apparently by making services available to those who are able to access them. Although, pressure may be reduced on government facilities, only the wealthier patients benefit from the private sector.

Nevertheless, some respondents have argued that dual practice has no benefits to the public system.

It does not have any benefits to the public healthcare institution; rather it is the other way around because most times the services in the public sector suffer. Doctors, who are supposed to be there to work in the public sector, abandon their work for the private sector. It would have been a benefit if those in the private remained in the private and those in the public remained in the public. But, as it is now, where those in the public sector abandon their work to go to the private, sector, it does not have any benefit. Ask any doctor who is a dual practitioner, the way they see patients in the public sector is different from the way they see them in the public sector. They are two quite different things (Interview with a non-DP Snr. Registrar)

This suggestion that there are no benefits was based on concern about the diversion of public patients to private practice and the general attitude of dual physicians who shirk their responsibilities in the public system for a private work.

\subsubsection{Benefits to patients}

The major benefits identified by respondents were access to equipped private sector, avoiding bureaucracy in the public sector, establishing doctor/patient relationship, proximity to patients and rendering quicker and better attention in the private sector. We consider each of these benefits below. 
Respondents discussed the benefit to patients in terms of access to better functioning equipment in the private sector. They argued that the public sector lacks modern equipment and patients are usually attracted to the sector to access superior equipment.

And, of course, what happened may be 20 years ago when public hospitals were no longer equipped or managed as they should be, so that you don't have equipment and you don't have facilities. Sometimes, it is not that the equipment is expensive; it is just the way resources are being allocated in the public sector. For instance, if a hospital is spending 98 or $99 \%$ of its subventions on salaries, it has only 2 or $3 \%$ for overheads to buy equipment. And, of course, you are also paying the salaries, meanwhile doctors and other members of staff do not have anything to work with. So, they will now go to the private hospitals which provide those equipment and facilities. This is because of what has happened in the public hospitals where the wage bill has grown so great that government is no longer able to buy or provide equipment. If it is running well, that may not be a factor that will... but I will tell you that, due to the lack of facilities in public hospitals, people now go to private hospitals where they can see a doctor (Interview with a non-DP Consultant)

In the private sector, some might have modern [or mobile] ultrasound, but if you go to some public hospitals in Nigeria, you might not find this so-called mobile ultrasound. These are some of the factors that drive people to the private sector (Interview with a non-DP Snr. Registrar)

Government hospitals lack modern equipment. Most of the existing ones are either old or not functioning efficiently. The allocation of resources to the healthcare sector, where the wage bill is high with little or nothing left to purchase equipment, was mentioned as one of the challenges facing public healthcare institutions. This situation seems to have accentuated the upsurge of patients to the private sector and why many public-sector doctors established private practices.

All the respondents saw bureaucracy in the public system as a problem that patients would try and avoid. Government hospital can be bureaucratic with many administrative hiccups that irritate patients. But, in private practice, bureaucracy is largely minimised, and patients can access a doctor quicker than the public hospital.

The benefits are great. If you have a hospital of your own, you can determine what happens, bureaucracy is minimised, and the patient can see you faster and they get what they need quicker. You are completely in charge and do not depend on anybody else. You are not depending on the finance office, records department, the pharmacy or the laboratory scientist. Whatever you can do, you do it quickly and 
the patient can benefit. So, it removes a lot of bottlenecks, a lot of bureaucracies, which, of course, we find in many of our public hospitals (Interview with a DP Consultant)

Yes, there are some patients that will come and tell you that they do not want to be in this public hospital and to ask if you have something more private. You were here now and a patient that was supposed to be operated on Wednesday looked for a bed space all over the hospital and could not find one. In this situation, if she has the money, because private hospitals are more expensive than the public, she will say to you, 'sir, I want this thing done quicker. I am travelling, and could there be any other way you can do it?' If you say no, because you do not want to have anything to do with a hospital patient, they may beg you and tell you that this is what they want. In a situation like that, they may probably use the private sector because it is faster. Like now, we have about thirty patients queuing up for surgery [elective cases] and then you must follow the protocol and, in a unit that operates two times in a week, if you do not operate this week, then it will be the next week. So, you keep on moving the patient. Right now, we have patients down for surgery up to as far as August 2016. But if, in a private practice, the patient comes, and you do your investigation, you simply do your surgery, and everybody is happy. That is an advantage (Interview with a non-DP Snr. Registrar)

Bureaucracy is characteristic of government institutions, including hospitals. It involves the official procedures or rules guiding how things are done in an organisation. Government uses it to ensure accountability and adherence to a set of rules. The application of bureaucracy in public hospitals causes delays and resentment among public patients and doctors. Many government-employed doctors established private practice to enable them to have a control over their practice and avoid government bureaucracy.

A closer patient/doctor relationship was viewed as typical of the private sector. According to the respondents, there is a one-on-one personal relationship between a patient and the doctor. This close interaction may be lacking in the public sector and that apparently reinforces the desire to visit doctors in their private practice.

Again, if you have a personal interaction with a doctor, the confidence building is more because, in a public hospital, there are too many patients struggling to see the doctor. However, in a private setting you can see this doctor at your own convenience, especially if you have some confidential questions to ask him (Interview with a non-DP Registrar) 
For me, I think that is a great advantage too. And then, in private sector, because there is more of a one-on-one relationship with a patient, management, to an extent, is more personalised or customised because, most times, it is arranged under the doctor's name which is known to the patient, and, therefore, the doctor is more careful in the sense that, if anything happens, if there is a litigation, it is directly against him or her. (Interview with a non-DP Consultant)

Because many doctors are involved in managing a single patient in public facilities the same close relationship with patients are less common than in the private sector.

Most of the respondents cited proximity as one of the benefits of dual practice to service users. They argued that most public hospitals are located far away from the people, but private clinics and hospitals are closer to patients' own neighbourhoods.

Dual practice is of benefit to the patients, the doctor and the hospital. How do I mean? In dual practice, sometimes this practice is located closer to the neighbourhoods of these patients; it is in their familiar environment. So, they do not have to go to faraway places to seek treatment. They just walk into the neighbourhood hospital and receive treatment and go back to their houses. If they have any complications, it is easy for them to access the hospital due to the proximity; it is a benefit to them. The promptness with which they are treated is a motivation for them (Interview with a DP Consultant)

Most of these teaching hospitals are far away. So, these peripheral hospitals help patients to get at least the initial care before you now start the main treatment. If you ban DP entirely, there is going to be a serious problem getting these patients to the public hospital in a timely manner, knowing the difficulties of public transportation in the country and many other things. For instance, what happened before was that it was difficult to move around. When you have that kind of situation, it will be easy to put someone in the car and drive to the nearest private hospital or clinic. (Interview with a non-DP GP)

Public hospitals are located far from many patients. In some instances, it could take a couple of hours to reach some tertiary hospitals; this can be a nightmare for many patients.

According to respondents, patients receive quicker and better attention in private practice because of reduced waiting time. It was mentioned that patients value time and, since there is a shorter wait-time in private practice, patients would, therefore, prefer to access those services. 
The waiting time is usually shorter in private practice than in a public facility because of a lot of protocols, and all the rest of it, and when they come to the private sector it saves a lot of lives. For instance, a woman had abruptio placentae and was rushed to the private hospital where I work, the baby was almost dead. I immediately got there and, within the next 20-30 minutes, that baby was out and, believe it or not, the mother was also alive. But if it were here [public hospital], the baby would have died because, by the time you finish making a diagnosis, because how we usually do calls here is that the junior doctors will first handle the patient, and then, if they cannot fix the problem, they call the senior doctors. If that immediate intervention was not given, of course, they could have missed it and the baby would have died. In fact, on two separate occasions, I have had that experience. If the patient was presented here [government hospital], forget it, the baby would have died. Deciding for an intervention process is always quicker in the private sector. There are a lot of bottlenecks here. If you call the anaesthetist to come and do the work, he will give you a lot of excuses, you ask a laboratory scientist to get the result for you, he will give you one week, but all this can be done in the private sector within minutes, you wait a while, get the results and you use them to manage the patient. (Interview with a DP Consultant)

As regards the patients, we have to analyse why they go to the private sector. First, is time; patients will tell you that they don't want to waste too much time. In the public sector, you waste a lot of time, you queue up, they send you for an investigation and the investigation is never out on time. Some people will tell you that our own is our own while my own is my own. (Interview with a non-DP Snr. Registrar)

Importantly, clinical decision-making is quicker and reduces the time for an intervention to be initiated. There may be too many protocols in the public system and the attitudes of staff in the delivery of care may seem different from that of the private sector.

\subsection{Policymakers'/stakeholders' perspectives}

\subsubsection{Benefits of dual practice}

Respondents talked of the benefits of dual practice mainly in terms of four sub-themes. These are benefits to the dual practitioner; benefits to patients, the idea that DP creates a market for the private sector and policy makers' perception of dual practice benefit.

\subsubsection{Benefit to the dual practitioner}

Respondents believed that the main benefit of dual practice for public sector doctors was to 
earn some extra income. This was seen as a simple and transparent motive that was understandable against the background of low public-sector salaries.

There is a limit to what a doctor working for the government earns. On the side of the practitioner, it enhances his earning and on the part of the entire society, it helps us to utilise what we have. On the part of the private sector, it enables us to offer services, which otherwise, we could not have been able to do because they recruit most of the doctors from the government sector who visit them in the evenings or in their free time (Executive Officer 1, Nigeria Medical Association).

And of course, on the other hand, the doctor who engages in dual practice at the right time gets remuneration to aid him (Senior Official 1, State Health Board).

Dual practice benefits the medical professional by augmenting his/her public-sector salary. In Nigeria, many pubic-sector doctors depend on this extra income to live more comfortable due to their supposedly poor pay. Their primary motive is to supplement government pay. Private practice seems to help them to use their professional skills to gain additional income, rather than do a different kind of job. However, it is also the case that by so doing, they may make their services available to a wider population.

\subsubsection{Benefit to patients}

This was another area where benefit was presented by almost all respondents in terms of a single consideration - the likelihood that paying for private care would give patients early access to expertise and medical facilities that would not have been available in a public hospital. The diversions of patients to the private sector help them to access well equipped hospitals in the private sector. Similarly, access to experts such as consultants and specialists who are few and may not be easily accessible in the public system can be more readily available in their private practice.

Many private hospitals are more equipped than the public ones. If the doctor is working in such a hospital and in a less well-equipped public hospital, he could do collaboration, he could help to get patients to benefit from the facilities in that private hospital. It will help for collaboration and transfer of expertise. (Executive Officer1, Nigeria Medical Association)

It enables people to access expertise, sometimes beating the bureaucratic chains in the public sector. (Senior Official 1, State Ministry of Health) 
Although respondents accepted that private hospitals often have better facilities there was a degree of scepticism about whether the diversion of patients was mainly for their benefit or for financial gain by the dual physician. One respondent reported that even a public teaching hospital known to him might sometimes refer patients to a private hospital to gain access to specialist diagnostic technology. At the other extreme examples were given of transfers of patients to private hospitals that lacked basic sterilisation equipment. However, it is the patient who is in the best position to voice whether s/he was more satisfied when diverted as compared with the services that would have been obtained in the public sector. While the benefits of patient diversion are mentioned positively by doctors, the negatives associated with this, such as loss of revenue to the public system and an increase in cost to patients are more often overlooked.

\subsubsection{DP is beneficial to the private sector}

The private market has benefited substantially from dual practice. According to respondents, the private sector hires experts from the pool of government-employed doctors. In addition, dual practice boosts the private market and there is a shared expertise across public and private sectors. However, in contrast to this stance, some respondents opposed the views that dual practice has any benefits.

There is an interwoven arrangement, even with the mission hospitals, owned by faith-based organizations and private hospitals, and it is still from the same pool of competent medical doctors that all these hospitals draw their health care professionals. (Executive Officer 1, Nigeria Medical Association)

Regulated dual practice is far better than a single practice - working just for government or private alone, because collaboration and sharing of facilities and expertise across the board instead of restricting everything to either the public or private sector. (Executive Officer 2, Nigeria Medical Association)

There is no doubt that dual practice is of benefit to the private sector. It helps to build a strong private sector and a strong private sector is beneficial for the healthcare system as a whole and therefore for the population it serves. Most competent doctors do not restrict their services to the public sector alone. Whether this is for financial gain or an act of professional altruism is debatable. Public sector specialists and consultants undertake most specialised services in the private sector, by so doing, services may be expanded. 


\subsubsection{Policymakers' perceptions of dual practice benefit}

Some high-placed respondents argued that DP has no benefits to the patient or the public system. Instead, only the dual practitioner benefits from the practice. To them, it only satisfies the financial drive of the doctor. They see dual practice as bad practice and regard it as selfish behaviour.

Dual practice is bad. Any person that is responsible knows that it is not fair. It is not fair to government and patients, it is only the DP that is benefiting, making his money and is happy (Director, Ministry of Health)

No, I do not think it is beneficial to the public health sector. Because if I engage a medical doctor and I expect him to work for 6 or 8 hours, and he is not doing that, instead, he is taking some hours out of the contracted work hours in the public sector to the private sector, I do not think it benefits even the patient or the public sector. It could benefit the individual medical doctor as an individual not the public health sector (Senior Administrative Officer, State Health Board)

None of the senior policy makers interviewed believed that dual practice is of any benefit to the public system. In their view it is only the practitioners that benefit from dual practice. They believe that if medicine is practiced as it should be there would be no need for patients to be moved from the public to the private sector. The loss of time and revenue to the public system because of dual practice would not arise. Despite this criticism, the public sector has shown ineptitude in handling dual practice.

\subsection{Chapter summary/conclusion}

The accounts from the three categories of the respondents and the survey results in this study show similar trend, although, with some mixed reactions on the benefits of dual practice in Nigeria. The Likert-type analysis shows speedier attention to patients in private practice than in the public system. Similarly, there is a reduced bureaucracy in private practice than what is obtained in government hospitals. Respondents were optimistic that DPs use private practice to save life during strike actions in public hospitals. The service users were positive that dual practice is beneficial to them. They hold the view that it affords them the opportunity for easier payment plan through the deferred payment system, which allows them to access services in DPs private clinic and make payment in instalments. The business-like type of services they receive in private practice makes it a better alternative to the public system. 
Doctors were also of the view that dual practice help patients have access to experts in private practice, and with DP, the bureaucratic process in government hospitals can be avoided. They went on to claim that extra income in private practice is necessary to supplement poor public salary.

Although, some doctors were quick to point out some benefits of DP to patients and the public system, some of their colleagues opposed such claims that DP is of any benefits to the public system. In as much as some policy makers agreed that DP were of some benefits to patients and the private sector, the senior administrators among them did not believe that DP can add any value to the public system.

The opposition of some senior administrators to the claim that DP is beneficial to the public system may have stemmed from the consequences perceived of DP in the public system in what might be a power tussle between the profession and the state. This is analysed in detail in the discussion. 


\section{Disbenefits of dual practice}

\subsection{Disbenefits of dual practice in the health sector}

\subsection{Disbenefits of dual practice to service users}

This chapter reports the findings on disbenefits of dual practice to service users. It narrates the Likert-type scale analysis of disbenefits of DP to service users. It further shows the impacts of DP in terms of burden on patient finances and how the referred patient group coped with paying for private treatment. The chapter also examines the impact of DP in terms of time loss to the public system through hours lost on absences due to engagement in private practice. It explores in detail the disbenefits of DP through the eyes of other categories of respondents and finally, a summary of the chapter.

\subsubsection{Descriptive Likert analysis of disbenefits of DP to service users}


Table 9: Disbenefits of dual practice to service users $(N=407)$

\begin{tabular}{|c|c|c|c|c|c|c|c|}
\hline Question & $\begin{array}{l}\text { Strongly } \\
\text { disagree } \\
\text { n(\%) }\end{array}$ & $\begin{array}{l}\text { Disagree } \\
\text { n(\%) }\end{array}$ & $\begin{array}{l}\text { Don't } \\
\text { know } \\
\text { n(\%) }\end{array}$ & $\begin{array}{l}\text { Agree } \\
\text { n(\%) }\end{array}$ & $\begin{array}{l}\text { Strongly } \\
\text { agree } \\
\text { n(\%) }\end{array}$ & $\begin{array}{l}\text { Mean } \\
\text { [SD] }\end{array}$ & $\begin{array}{l}\mathbf{9 5 \%} \\
\text { CI } \\
\text { (lower- } \\
\text { upper) }\end{array}$ \\
\hline $\begin{array}{l}\text { 33a). Government doctors } \\
\text { engaged in private practice } \\
\text { come late to work in } \\
\text { government hospital }\end{array}$ & $4(1.0)$ & $46(11.3)$ & $28(6.9)$ & $192(47.2)$ & $137(33.7)$ & $4.0(01.0)$ & $3.9-4.1$ \\
\hline $\begin{array}{l}\text { 33b). Government doctors } \\
\text { engaged in private practice } \\
\text { are usually absent from work } \\
\text { in government hospital }\end{array}$ & $26(6.4)$ & $53(13.0)$ & $36(8.8)$ & $180(44.2)$ & $112(27.5)$ & $3.7(1.2)$ & $3.6-3.9$ \\
\hline $\begin{array}{l}\text { 33c). Doctors engaged in } \\
\text { private practice divert patients } \\
\text { from government hospital to } \\
\text { their private practice }\end{array}$ & $34(8.4)$ & $46(11.3)$ & $45(11.1)$ & $146(35.9)$ & $136(33.4)$ & $3.8(3.8)$ & $3.6-3.9$ \\
\hline $\begin{array}{l}33 \mathrm{~d}) \text { Government doctors } \\
\text { engaged in dual practice give } \\
\text { poor service to patients in } \\
\text { government hospitals }\end{array}$ & $36(8.9)$ & $126(31.2)$ & $19(4.7)$ & $169(41.8)$ & $54(13.4)$ & $3.2(1.3)$ & $3.1-3.3$ \\
\hline $\begin{array}{l}33 \mathrm{e}) \text {. Cost of care is not } \\
\text { affordable in private practice } \\
\text { of government doctors }\end{array}$ & $7(1.7)$ & $27(6.7)$ & $15(3.7)$ & $184(45.5)$ & $171(42.3)$ & $4.2(0.9)$ & $4.1-4.3$ \\
\hline $\begin{array}{l}\text { 33f). Government doctors } \\
\text { spend shorter time with } \\
\text { patients in government } \\
\text { hospital than in their private } \\
\text { practice }\end{array}$ & $52(12.8)$ & $98(24.1)$ & $26(6.4)$ & $124(30.5)$ & $106(26.1)$ & $3.3(1.4)$ & $3.2-3.5$ \\
\hline $\begin{array}{l}33 \mathrm{~g}) \text {. Government doctors } \\
\text { first attend to patients in their } \\
\text { private practice before } \\
\text { coming to government } \\
\text { hospital }\end{array}$ & $8(2.0)$ & $24(5.9)$ & $48(11.8)$ & $144(35.4)$ & $183(45.0)$ & $4.2(1.0)$ & $4.1-4.2$ \\
\hline
\end{tabular}

Scores ranged between 1 and 5

Patients' views of the disbenefits of DP are summarised in the above Table. Lateness to work has been identified as one of the key disbenefits of DP. There was a majority perception that dual practitioners report late to work in government hospitals with $80.9 \%$ agreeing. This may suggest that DPs will first attend to their private patients before reporting to government hospitals, something agreed by an overwhelming majority (80.4\%) of respondents. A key risk associated with lateness for work in government hospital is that it might result in a shorter time spent with patients - a factor viewed as a disbenefit of DP by more than half of the 
respondents, with $56.6 \%$ agreeing. Another significant related issue is absence from government work, which an overwhelming majority (71.7\%) agreed was a problem. On the issue of cost of care, an overall majority of respondents, $(87.8 \%)$ shared a concern that private practice costs were higher. On the other hand, poor service to patients was more common in public practice with slightly over half $(55.2 \%)$ of respondents agreeing that was the case. The same was applicable to patient diversion with a significant number $69.3 \%$ agreeing that public patients are often diverted to private practice.

It is no surprise that cost of care in private practice was noted as a key disbenefit of DP by a majority, especially in Nigeria where a significant number of people do not have health insurance but rely on out-of-pocket spending. The attitudes of DPs who first attend to private patients before reporting to government hospitals where they have full-time contracts seems worrisome to patients who must wait helplessly in government hospitals. Lateness, absence from work, and patient diversion are some of the disbenefits of DP to service users. Although the distribution of responses between 'strongly agree' and 'agree' in 'DPs first attend to private patients' comes with a higher mean, there was a marginally higher percentage of respondents who agreed that 'lateness to work' was significant.

\subsection{Impacts of dual practice in terms of the burden on patients' finances}

This section focuses on the findings related to the impacts of dual practice on patient finances. It covers patient expenditure for treatment in the private sector when referred from the public system. The findings included the estimated mean cost of treatment in public hospitals for the same health conditions for which patients were transferred to the private sector. Result on self-rated financial impact of private treatment for the patient group referred from the public system was also obtained. Finally, we found the coping strategies for paying for private treatment by the patient group transferred from the public system.

\subsubsection{Cost of treatment in private hospitals when referred from the public system}


Table 10: Respondents' expenditures for treatment in private hospitals as a result of referral from public hospitals $(n=34)$

\begin{tabular}{llllll}
\hline Category of cost & $\begin{array}{l}\text { Cost in }\left(\mathbf{N}^{*}\right) \\
\text { Min-max }\end{array}$ & Mean (SD) & Median & Range & IQR \\
\hline $\begin{array}{l}\text { Transport to private } \\
\text { hospital }\end{array}$ & $0-2500$ & $536(622)$ & 250 & 2500 & 900 \\
$\begin{array}{l}\text { Transport from } \\
\text { private hospital }\end{array}$ & $0-2500$ & $492(566)$ & 250 & 2500 & 900 \\
Cost of hosp. card & $0-5000$ & $1851(1454)$ & 1500 & 5000 & 1713 \\
Consultation fee & $0-20000$ & $3079(4020)$ & 2000 & 20000 & 3500 \\
Cost of drugs & $0-200000$ & $17540(34407)$ & 8250 & 200000 & 11300 \\
Cost of lab test & $0-26000$ & $3303(4983)$ & 2000 & 26000 & 4500 \\
Cost of X-ray & $0-15000$ & $1882(3843)$ & 0.00 & 15000 & 2250 \\
Other costs & $0-75000$ & $2456(12860)$ & 0.00 & 75000 & 0 \\
Total mean cost & $1250-215500$ & $32104(39738)$ & 22100 & 214250 & 23400 \\
\hline
\end{tabular}

Respondents answered all questions, *N=Nigeria Naira

The above Table shows that mean cost of drugs for all patients referred from the public hospital was N17540. The mean costs of transportation to and from private hospital were N536 and N492 respectively. Cost of hospital card/registration was N1851, consultation fee (N3079), cost of laboratory test (N3303), cost of X-ray (N1882). The total mean cost of treatment in private hospital as a result of referral from the public sector was N32104.

\subsubsection{Estimated mean cost of treatment in public hospitals for the same health conditions for which patients were referred to the private sector}


Table 11: Estimated mean cost of treatment in public hospitals for the same health conditions for which patients were referred to the private sector $(n=34)$

\begin{tabular}{llllll}
\hline Category of costs & $\begin{array}{l}\text { Cost in (N) } \\
\text { Min-max }\end{array}$ & Mean (SD) & Median & Range & IQR \\
\hline $\begin{array}{l}\text { Transport to private } \\
\text { hospital }\end{array}$ & $0-1000$ & $314(330)$ & 200 & 1000 & 300 \\
$\begin{array}{l}\text { Transport from } \\
\text { private hospital }\end{array}$ & $0-1000$ & $298(308)$ & 200 & 1000 & 300 \\
$\begin{array}{l}\text { Cost of hospital card/ } \\
\text { registration }\end{array}$ & $0-800$ & $345(198)$ & 400 & 800 & 350 \\
$\begin{array}{l}\text { Consultation fee } \\
\text { Cost of drugs }\end{array}$ & $0-1000$ & $76(233)$ & 0.00 & 1000 & 0 \\
$\begin{array}{l}\text { Cost of lab test } \\
\text { Cost of X-ray }\end{array}$ & $0-100000$ & $6074(17530)$ & 1500 & 100000 & 4750 \\
Total mean cost & $0-5000$ & $2236(5438)$ & 1000 & 30000 & 1850 \\
& $160-115900$ & $\mathbf{9 9 6 0}(\mathbf{2 1 5 0 4})$ & 4550 & 115740 & 8980 \\
\hline
\end{tabular}

Respondents answered all questions

In Table 11, the estimated total mean cost of treatment in public hospital for patients referred from the public system was N9960. Other cost categories include cost of transportation to and from public hospitals were N314 and N298 respectively. Cost of hospital card/ registration was (345), consultation fee (N76), cost of drugs (N6074), and cost of laboratory test N2236. 
Figure 4: Compares mean cost of treatment for the same health conditions of patients referred from the public system to private sector

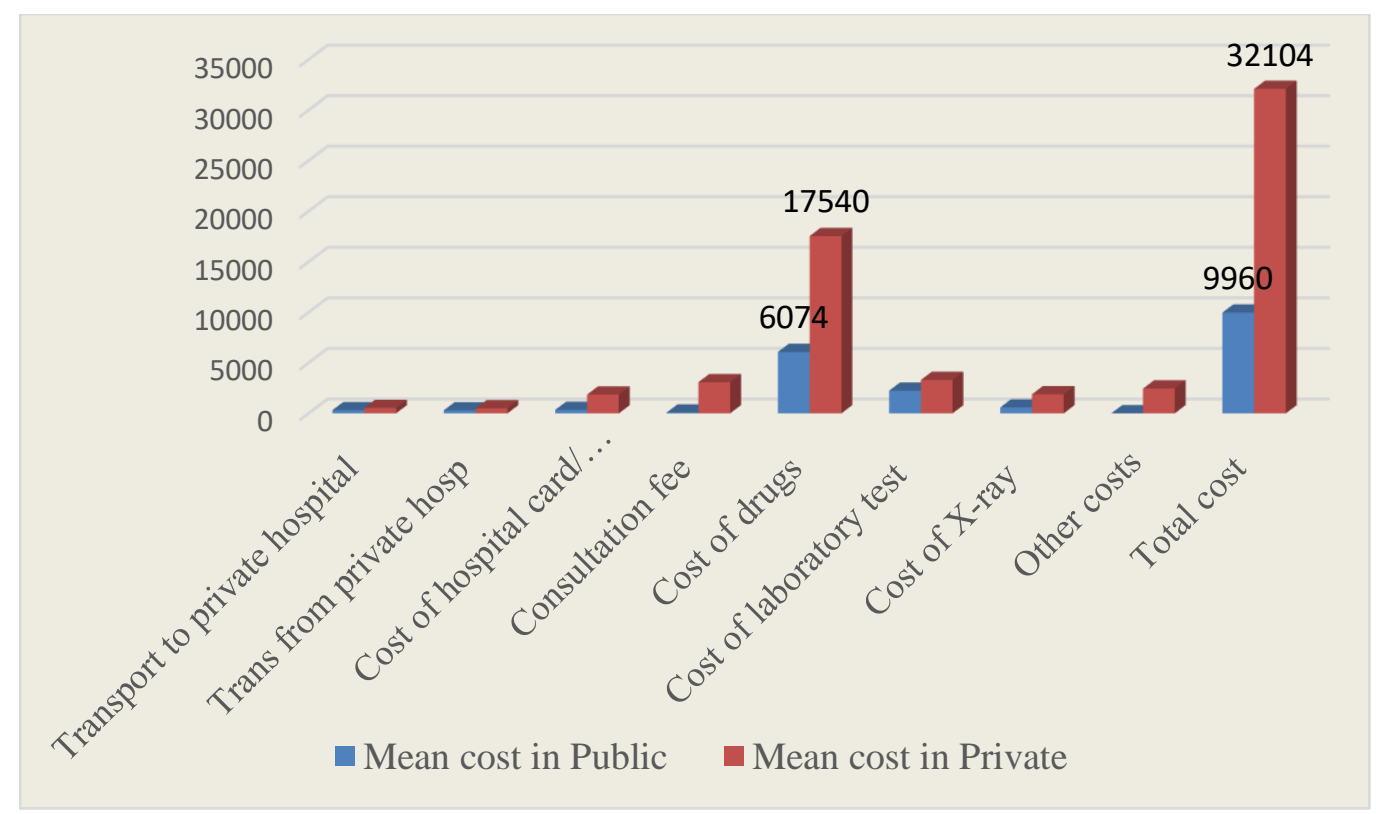

Figure 4 compares total mean cost of treatment in private hospital N32104 (US\$105) with that of the public sector N9960 (US\$33). The same applies to mean cost of drugs, whilst the private care cost N17540 (US\$57), the public care was N6074 (US\$19.8).

Table 12: Self-rated financial impact of private treatment for patient group referred from public hospitals $(n=34)$

\begin{tabular}{ll}
\hline Variables & $\mathrm{f}(\%)$ \\
\hline No impact & $6(18)$ \\
Moderate impact & $12(35)$ \\
Serious impact & $9(27)$ \\
Very serious impact & $7(21)$ \\
Total & $34(100)$ \\
\hline
\end{tabular}

Percentages rounded as small numbers

As Table 12 above shows the degree of, self-rated financial impact of private treatment is spread across the spectrum with no clear pattern. Just over half of those referred experience no impact or only moderate impact, while a significant group suffer impacts that they consider to be serious or very serious. Given the small size of the referred group in this study, 
that pattern must be regarded as only a tentative finding, and this is an area deserving of future research.

\subsection{How did referred patient group cope with paying for private treatment?}

This section shows how patients paying for private treatment when they were referred. The different strategies used as coping strategies were own money, borrowed money, sale of household moveable assets, sale of family land, someone else paid, and reduction of bill by the doctor.

Table 13: Coping strategies for paying for private treatment by the referral group $(n=34)$

\begin{tabular}{ll}
\hline Variables & $\mathrm{f}(\%)$ \\
\hline Own money & $31(67.4)$ \\
Borrowed money & $9(19.6)$ \\
Sold household asset & $2(4.3)$ \\
Sold family land & $1(2.2)$ \\
Someone else paid & $1(2.2)$ \\
Bill was reduced by doctor & $2(4.3)$ \\
Total & $46(100)$ \\
\hline
\end{tabular}

Some respondents identified more than one relevant coping strategy

This study attempted to shed more light on the impacts of referral by asking referred service users what coping strategies they used to pay for private treatments (Table 13). A majority 31 of 34 service users- said they had used their own money. However, this appears to have been supplemented in some cases by money from other sources as 9 of the 34 reported having had to borrow money. A few individuals had sold property, got assistance from another person or negotiated a reduction in the bill with the doctor. 


\subsection{Impacts of dual practice in terms of value of hours lost to the public system}

In this section, we present hypothetical estimates of the magnitudes of value of hours lost to the public system because of absences due to DP. To achieve this, a series of hypothetical scenarios were used to estimate the value of hours missed due to absences from the normal routine hour duty of 40-hour per week in the public sector. Based on the accounts of different categories of respondents in this study of doctors' absences in the public sector, these estimates would help to provide data on the size of resources outflows from the public system due to DP. These losses therefore are calculated based on the hypothetical $5,8,10,12,15$, and 20 hours loss scenarios per week per year in a typical tertiary hospital in Nigeria. This section focuses on two areas - the normal duty hours lost to the public system due to dual practice and the on-call hours lost due to dual practice. A combined estimate of different scenarios of the value of hours lost on normal duty hours are presented below.

The estimates of the value of hours lost are made for staff in one tertiary hospital. This is because all federal health institutions use the Consolidated Medical Salary Structure for their doctors and dental officers. Therefore, there was no need to include multiple hospitals since the parameters used for the calculation were the same. 
Figure 5: Combined hypothetical estimates of total value of hours lost for weekly absences for normal routine hours

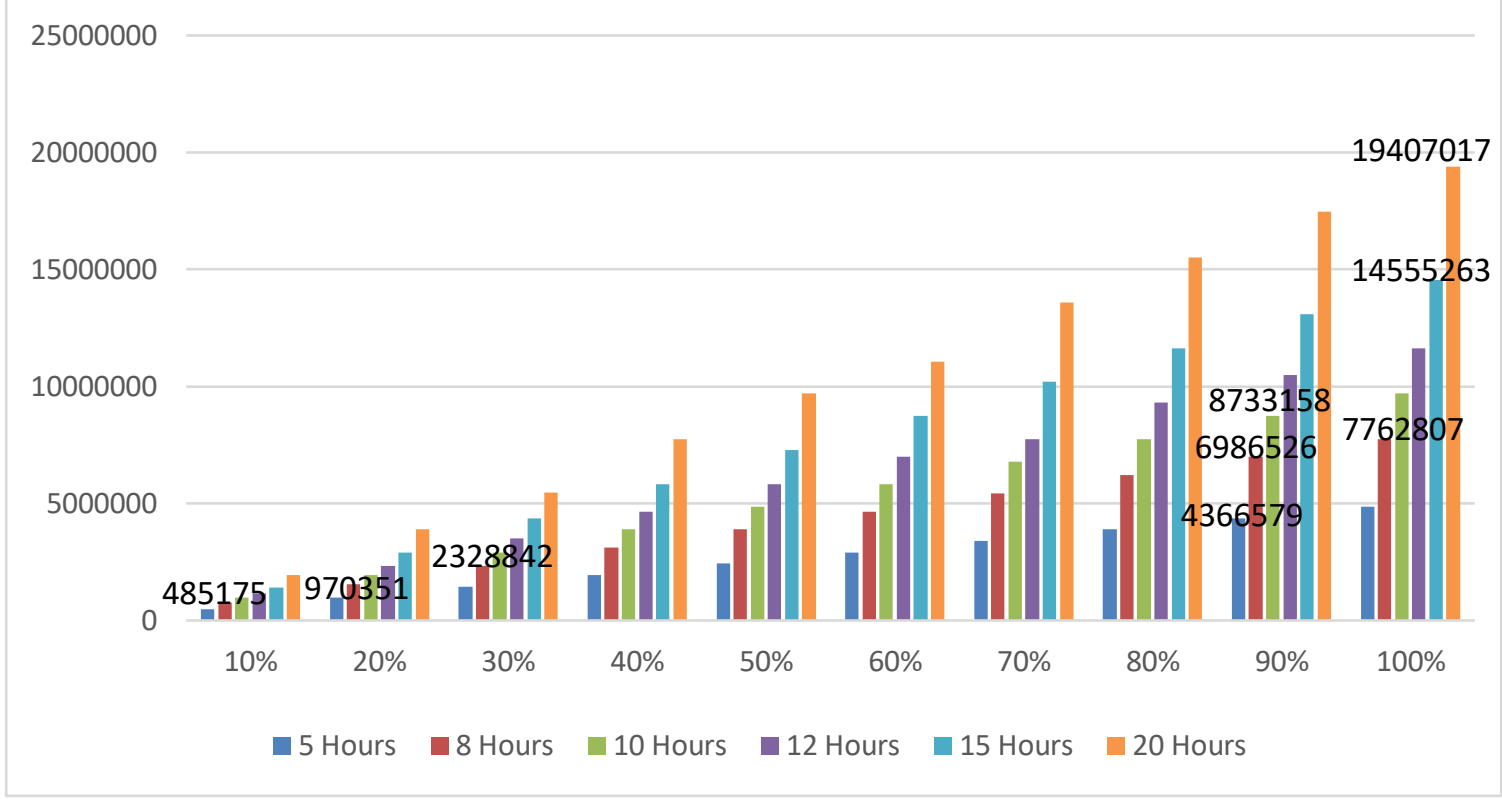

Exchange rate used: 1Naira= USD306 (Source: Central Bank of Nigeria, 2017)

In Figure 5 above, the value of the hours lost if $10 \%$ of doctors engage in dual practice and are absent for five of their contracted routine hours is N485,175 or 1,586 USD per year. As the number of doctors engaged in DP increases, so the value of hours lost to the public sector rises. Assuming that $20 \%$ of doctors engage in DP and are absent for five hours per week, the total value of hours lost amounts to N970,351 or 3,171 USD per year. With 30\% of doctors engaged in DP and absent for 8 hours per week, N2,328,842 or 7611 USD would be lost. If 90\% were involved in DP, the total value of hours lost would increase to N4,366,579 or 14,270 USD for five hours of weekly absences, N6,986,526 or 22,831 USD for 8 hours of weekly absences, and N8,733,158 or 28,540 USD for 10 hours of weekly absences, and then N14,555,263 or 47,566 USD for 15 hours of weekly absences if $100 \%$ were involved in DP. At the extreme twenty hours of weekly absences with a $100 \%$ of the doctors engaged in DP would result in a loss of $\mathrm{N} 19,407,017$ or 63,422 USD to this single tertiary hospital. The value of hours lost to weekly absences from the routine hours for the different cadres of doctors in the various scenarios can be found at appendices (see 12-17). 


\subsection{Hypothetical estimates of on call hours lost to dual practice and financial implications in a tertiary hospital}

This section deals with the estimates of on-call hours that could be lost to the public system due to absences resulting from DP. It uses a series of hypothetical scenarios to estimate the values of hours lost due to on-call hour absences. Apart from the normal 40-hour per week duty hours in the public sector, doctors in tertiary hospitals will also spend periods "on call" to deal with emergencies in addition to their normal routine hours. Doctors are paid an on-call hour allowance alongside their main salaries. When attendance to cover on-call hours is compromised due to private practice, this therefore, involves additional hours lost to the public system. Estimating the magnitude of value of hours lost, helps to provide a clue to the size of resources outflows from the public system. These losses are calculated based on hypothetical 5, 6, 7, 8, 9, and 10 hours loss scenarios per week per year in a typical tertiary hospital in Nigeria. 
Figure 6: Combined hypothetical estimates of value of hours lost for weekly absences for on call hours

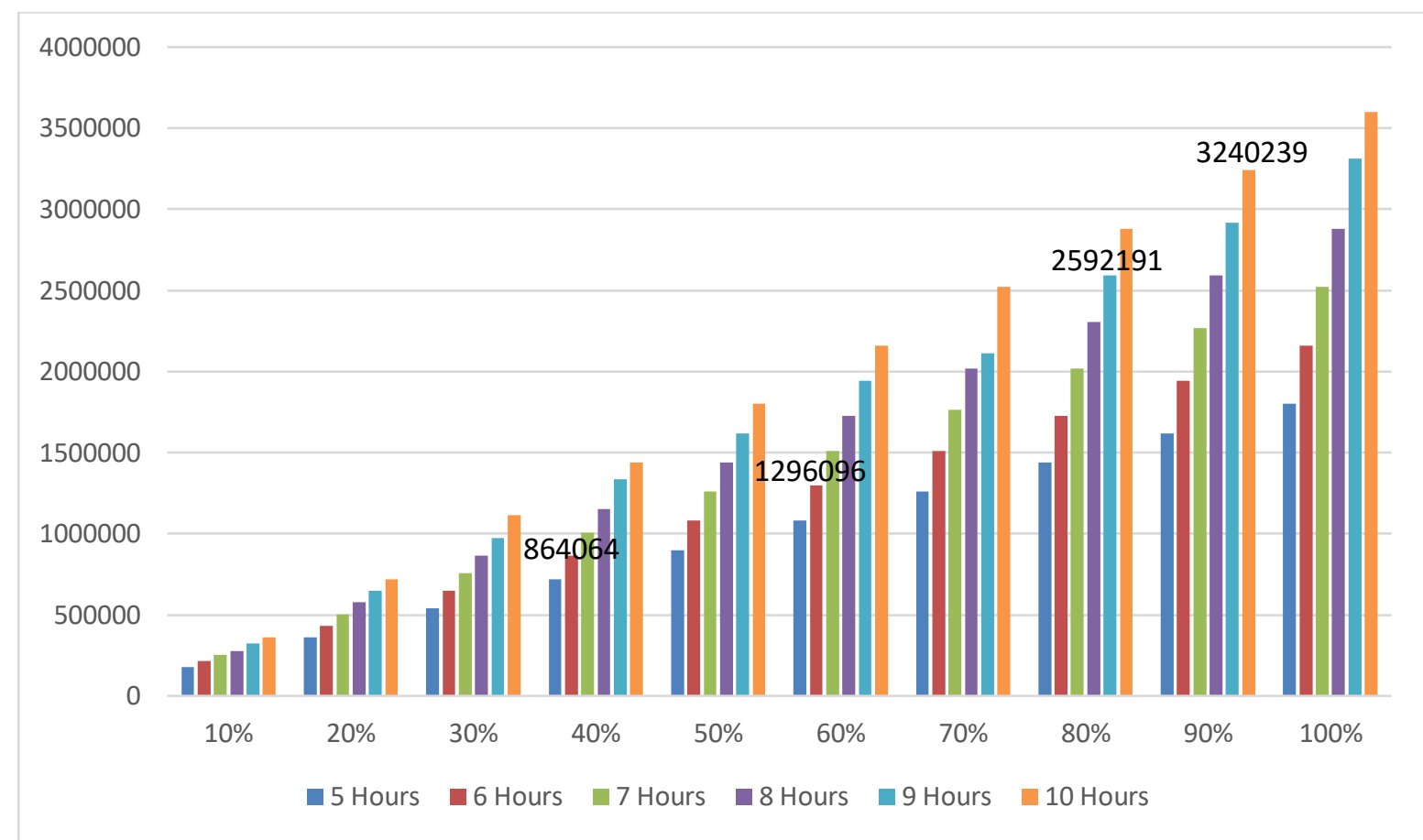

In Figure 6, the total value of hours lost from on call hours when $40 \%$ of the doctors engage in DP with 6 hours weekly absences would be N864,064 or 2,824 USD annually. As the number of doctors engaged in DP increased to $60 \%$, the total value of hours lost to the public system for 6 hours weekly absences from on call hours would increase to N1,296,096 or 4,236 USD per year. Similarly, the total value of hours lost to on call hours at 9 hours weekly absences assuming $80 \%$ of the doctors engage in DP amounts to N2,592,191 or 8,471 USD per annum. If absences from on call hours rise to 10 hours and $90 \%$ of the doctors engage in DP the value lost to the hospital rises to N3,240,239 or 10,589 USD per year. See appendices (19-24) for the value of hours lost to weekly absences from on call hours for the different cadres of doctors for the various scenarios.

\subsection{Disbenefits of dual practice through the eyes of respondents}

This section narrates the findings from the qualitative data obtained from different categories of respondents - service users, policymakers/stakeholders and doctors. It covers the disbenefits of dual practice to the health sector of doctors who work for government and 
operate private practice. Findings from each of the categories of respondents are presented below.

\subsubsection{Service users' perspectives on disbenefits}

In this section, four sub-themes emerged from the service users' perspectives on disbenefits of dual practice. These are the loss to the public system, extra cost to patients, low commitment to public patients, and public hospital work. Each of the sub-themes is explained below.

\subsubsection{Loss to the public system}

The participants were of the view that dual practice is not without financial loss to the public system. This loss was found in the form of perceived pilfering of public sector equipment and drugs to the private sector by the dual physicians. There is a loss of revenue when public patients are self-referred to private practice.

Some of the 'interview extracts' from the participants help to add more insight

Self-referring patients will result in a financial loss to government. The money a patient would have paid to government hospital is paid to the doctor in his private practice. The dual practitioners also divert government hospital equipment to their own private practice. (FGD1, P.6)

When my father was sick I was with him for about two months. The first hospital we visited was the general hospital X in Lagos. After three days, we were referred to another bigger general hospital because of a lack of equipment in the first hospital. We spent three weeks in hospital X, and after several tests, the problem was diagnosed. The consultant then referred us to his own private hospital and gave us a referral paper to go to a private laboratory for another test. (FGD 2, P.2).

The diversion of public patients, equipment or drugs from the public system to private sector results in a loss to the public system. Such loss could be a gain in the private sector, but perhaps to the dual physician, and those who can access private services. Some have argued that private patients gain as well, but not without extra cost. Some studies have shown that revenue loss from the public system to the private sector occurs in the form of absenteeism, equipment and drug diversion. There is a perception that government indirectly finances the private sector when its full-time medical professionals who earn full-time income from the public system, acquired skills and training using government finances and equipment, now 
transfer those skills to the private sector at a loss to the public system. Therefore, understanding who gains in such practice has an implication for policy on dual practice in Nigeria.

\subsubsection{Extra cost to patients self-referred by the DP}

Data revealed that patients pay extra when self-referred from public to private practice. Majority of the participants have been self-referred in the past year. The dual practitioner selfrefers patients to their private practice or use 'agents' such as nurses or other healthcare workers to divert patients to their private clinics. Such diversions could make patients lose opportunities for services in the public sector, which they would have obtained, perhaps at a minimal cost.

I was referred to a dual practitioner's private practice by his colleague in the government hospital. When it was my turn to see the doctor, they called me from the General Outpatient Department (GOPD) to enter the doctors consulting room. When I got there, and explained my condition to her, she told me that she had understood everything I said. She told me that she would refer me to a doctor in a private hospital who could handle my case. But she turned and asked me if I am happy with what she said. I said yes that I am happy. So, she called the doctor and told him that she is sending someone to him. She took me in her vehicle to the hospital [government] gate from where I took a bus to the private hospital. That doctor in the private hospital wrote some drugs for me using his hospital letter head paper and then told me where to go for the laboratory test and where to buy the drugs. You must go to the specific places he asked you to go for your laboratory test and drugs, if not, the drugs you bought may not be administered on you. You also must pay for the prescription he writes for you. (FGD 4. P3)

Patients pay extra in the private sector when they are diverted from the public sector. Some have argued that dual practice gives the patient access to consultants who might not be readily available in the public sector. The practice of diverting patients from the public system may suggest that financial interest as against professional altruism seems to influence self-referral. Although, for many of the respondents, patient diversion is an indictment of the public system, for failing to protect public patients from the overbearing power of the professionals by its inability to introduce measures against the self-referral practice. 


\subsubsection{Low commitment to public patients}

Our participants were of the view that they do not get the desired attention from public sector doctors. Their point of view centred on negligence affecting patients, and a lack of courtesy from the dual physicians. These attitudes according to them are not exhibited in their private practice.

...the doctors in the government hospital do not give good attention to public patients. They also ask you to come to their own private hospital. I have experienced it. The last baby I had in a government hospital died. One of the pregnant mothers that came for antenatal told me that one of the doctors has a private hospital and why did I not go there. The doctor did not come to the hospital on time as there was no body to monitor his attendance to work. I was there almost dying before I was rushed to a private hospital. If you meet these doctors in their private hospitals, you will be surprised how they will attend to you. You may be wondering if these doctors are the same people that you see in the government hospitals. So, they give you attention in their private hospitals because the money they make belongs to them. In the government hospital because there are no checks, they tend to do whatever they want. Sometimes they use words that are discouraging on you with disregard; after all, you will still come back to the hospital. But in their private hospital, which is their life investment they will treat you as though you were a king. In the government hospital, they use vulgar words on someone who is dying. But in their own private hospitals they use good words so that you come back next time and pay them good money. (FGD 1. P3).

A Government doctor that has a private practice may not have enough time to give you the desired attention in government hospital. Like what I have experienced, the doctor told me that if I wanted more attention I should come to his own clinic. (FGD3, P.1).

There seems to be a conflict of interest when a doctor holds two similar jobs that are competitive in nature. While it may be plausible to argue that such practice expands access to healthcare service, its potential conflict of interest may far outweigh any advantage. On the other hand, poor attention to patients in public hospitals could also mean an indirect way of diverting them to private practice where full attention may be given. The supposedly good attention to private patients may go beyond the interest of patients' welfare to interest in their finances and intend to retain them for future healthcare transaction. 


\subsubsection{Low commitment to public hospital work}

The study also found a perception that DPs have low commitment to public work. DPs have been reported of coming late to work. The participants' experience showed that DPs first consult in private clinics before reporting to public work. Absence from work was also noted among them.

Our participants recounted the nature of low commitment to public hospitals by DPs in different ways.

Another disadvantage of dual practice is time. You know you cannot divide love. Likewise, you cannot divide time. For instance, as I am here, I cannot be in my house. I cannot divide myself. These doctors will spend time in their own private clinics and will not come to government hospital on time. They may stay in their private hospitals from 8 am to 10 or $11 \mathrm{am}$, and when they come to government hospital, they stay for about one or two hours and leave. And those medical students they are supposed to teach during consultation, you see them wandering about because there is no one to teach them (FGD 4. P.2).

I think the issue of dual practice of medical professionals should be investigated. They give an unequal attention to both sides. They spend few hours in the government hospital and more time in their private practices. They give less attention to government hospital but collect more income. This should be investigated (FGD1, P1).

It may be practically difficult to give equal attention to both public and private work, assuming more income comes from private work. Therefore, public patients may be neglected, and rights of patents may rarely be met in public hospital when attention is shifted away from them. Ownership of private clinics and hospitals by consultants and senior medical officers may likely "crowd out" the doctor's effort in the public sector, though; the main strand of argument by doctors for low commitment is poor salary in the public sector. Low commitment to public work by DPs may have wider explanation. The overall health system context may have been weakened by austerity and poor implementation of DP policy in the country. Many doctors, therefore, would like to augment their salaries with additional private income. Though this seems justifiable, the impacts to the public healthcare delivery remain a priority. The use of the public system's resources for improving the private sector especially 
with public sector human resources could have an implication for an optimal service delivery in the public system.

\subsection{Doctors' perspectives on disbenefits of DP}

This section is structured under four sub-themes on disbenefits of dual practice. Discussions and responses about disbenefits of dual practice were specifically centred on disbenefits to public patients, how dual practice affects medical training in government hospitals, low productivity by doctors in public hospitals and indulgence in sharp practices in private practice. Each of these sub-themes is discussed below.

\subsubsection{Disbenefits to patients}

Respondents suggested that patients pay more due to the nature of services they receive in the private sector. Take for instance, the issue of a CT scanner, which may be rarely seen in the public system, but it is available at high cost in the private sector.

The cost of healthcare is cheaper in the public sector, but very expensive in the private sector. In the private sector, you may know what to do to give the patient the best, but those things may not be there in the public sector. Take, for instance, the CT scanner; in the western world it is something you do every day [a routine use of CT scanner]. But, in Nigeria, it is not that easy. If you go to a private sector, it will cost you more, but here, in the public hospital, like in Enugu, you rarely see a CT scanner in our public hospitals. So, financially, it is cheaper to get your medical attention in a public hospital rather than in the private sector where it is expensive (Interview with a non-DP GP).

It is quite unlike the private sector where services seem to be almost automatic. But for one to get that automatic-like care service you have to pay the cost. So, in terms of benefit to the patient, the financial constraint is lower in the public sector. (Interview with a non-DP Consultant)

The choice of care for the poor SES patients seems limited, thus, they patronise the public system.

\subsubsection{Dual practice affects medical training in the public system}

Some doctors were concerned that dual practice could put the future of medical training in Nigeria in jeopardy. Respondents suggested that because dual practice reduced the flow of 
patients to the public sector as the numbers of private clinics and hospitals grow, the wide case mix necessary for training may not be present.

The way we function here is that, once you are employed, your services should be available 24/7. But it doesn't work. This is because each person has his own unit somewhere in town where he sees his private patients; you may be amazed at the type of surgery that is done in private practice. How will you now get to train residents? The residents are not helping matters because they also open their own little shops where they do private practice; God knows what they do there. So, it is a cycle that it is bound to...it is a self-destructive cycle, I am afraid (Interview with a non-DP Consultant).

The time given as trainers by senior doctors is reduced.

...the training also may suffer because the trainer may not always be available; the patients may not be coming to public hospitals and may begin to gravitate towards private hospitals where someone who has the skills may offer it and two; there will be promptness of service. Therefore, the trainee may not be able to see all that they are ordinarily supposed to see and also treat or participate in treating, so that they can be better specialists when they graduate. So, those are the major things that I consider important in that people may not offer as many of the services they may need to offer, and experiences may be fewer for the trainee (Interview with a DP Consultant).

Private practice may contribute to the non-availability of trainers in the tertiary hospital training centres, which may compromise the quality of the training and poses challenges to its future sustainability.

Regarding the impact on patient flows respondents said the following:

Now, what is the net effect of that [private practice]? The net effect is that the training in our tertiary institutions or training centres is being compromised. The training is being compromised by the incessant strikes and the non-availability of patients in the training centres because they go to the private hospitals (Interview with a non-DP Consultant).

On the other hand, if the public hospital is not busy, it may suffer from patient drought [lack of patients], patients will be leaving, or, if their practices are not in tune with what patients want, they will be having a low patient turnover, and, as a result, teaching of medical students, nursing students and postgraduate doctors will then suffer. Because, for you to teach them, you need to have a good number of cases to demonstrate, to practise and to operate and show them, and they need to learn by seeing diverse cases, unlike when they are seeing just a handful of 
cases. It also has some negative side effects. It can deprive government hospitals of core patients to train with (IDI with a DP Consultant).

\subsubsection{Low productivity in the public sector}

Low productivity in the public system was viewed as a challenge to optimal service delivery. A number of issues were raised by respondents. The rate of absence and late coming to work among government doctors was seen to be problematic. Additionally, conflict of interest between public sector work and private practice was raised as a challenge confronting the public system. Underperformance in the public system was mentioned as a concomitant of dual practice.

Several respondents mentioned attendance at work as a problem.

Sometimes, because there are no checks and balances from the supervisory bodies, they tend to, ... we see cases where doctors do not come to work on time; and even when they do come, they spend only a short time there and then leave and they will give all their time to the private sector. Things like that are conflicts of interests, which need to be checked (Interview with a non-DP Consultant).

...no, I do not think so. It may be so in some centres, but not in this hospital. I do not want to mention names because you are recording things, but there are some hospitals in this country where there may not be much regulation and some where they do as they please. Sometimes, they come to work by 12 or 1pm and leave by 2 or 3pm. People just zoom in and zoom out, that is what I call it. (Interview with a DP Consultant)

It is always said that it is in our time that people have stopped working. You see people who are consultants, they do not come to work, there is really no punitive action and neither do their consciences flog them nor is there any external person flogging them. And some of these people are senior to you, they trained you and what regulatory measures do you take, do you jail them, do you stop their salary? It is very difficult. I don't know what rule will help solve the problem when rules are made without enforcing them (Interview with a non-DP Consultant).

The problem of poor time-keeping seems common among senior doctors. It shows a poor work attitude in the public sector and also demonstrates the weak supervisory role of the MoH. It has many consequences, such as poor patient management, poor resource utilisation and both patients and the public system may suffer. 
General opinion was that private practice will always thrive to the detriment of the public sector. The dual practitioner tends to give more time and attention to their private practice than the public system where they have a primary full-time contract.

[Laughs], ok, it happens. That is why I said that that private work eats deep into your time. I can tell you that it is subjective. It depends on the individual. Some may ask their patients in private practice to come to the government hospital where they are working. But some will also abandon their work in the public sector and rush to their own private set up to attend to a patient. So, it is a twoway thing and depends on the individual. I can tell you that the majority will abandon their work in the public to go and attend to their private patient in the private clinic in an emergency, provided you have someone who might be able stand in for you in the government sector, which, of course, is almost always available if you have something urgent to attend to. That is what many of them do (Interview with a DP Snr. Registrar).

You cannot serve two masters and love them equally...You see the private sector is more in the heart of private practitioners than the public one. They see it as their brainchild and it is closer to them than the public sector. The public sector is owned by everybody and it is not your thing. So, most times you see people robbing public hospitals of time, its workforce and everything. (Interview with a non-DP Snr. Registrar)

The dual practitioner may most likely give priority to their private work rather than the public.

It is difficult to serve two masters at the same time; one certainly may suffer at the expense of the other. The public system might be the loser in this case, because attention may be shifted to private work.

One recurrent theme was that in many cases the services doctors render to the public system does not match the pay they draw.

In fact, they are many doctors who just draw their salary and they are not doing any work. They may feel that their salary in the public sector is guaranteed and you see them give all their attention to their private practice. There are a lot people like that and there are a lot of centres where it is like that. There is no justification for that. It is not supposed to be like that. People who are in the public service should provide the services for which they are needed and, if you are not satisfied, you leave it and go private. (Interview with a non-DP Consultant) 
The dual practitioner, well I am not quoting studies, but we live in an environment where we observe what is happening, and for an unregulated system like ours the dual practitioner does not give adequate services in the government sector for what he is currently paid. (Interview with a DP GP)

Underperformance by DP physicians may indicate that they invest more time in their private practice than the public system. They receive the salary, but do not perform the work. In an environment where there is weak regulation, as it is in Nigeria, it may be difficult for a dual physician to give a full commitment to government work.

\subsubsection{Sharp practices in the private sector}

Lack of peer review in private practice has been argued to be a major disadvantage of dual practice. The practitioner takes all decisions concerning the patient with no inputs from colleagues. Some of them have been accused of dabbling into areas they are not trained in, just to get the financial reward.

In DP, even a doctor who is not specialised in a given area can dabble in another area that is what I call sharp practice. You do not have that specialisation of duties, quite unlike the public hospitals, where you do not go into an area that is not your own. I am a paediatrician, I do not go and handle gynaecological care and I do not go into surgery. In a private set up, I even know some of my colleagues who are paediatricians, but they do also surgery [CS]; if you go to the private hospitals, there are lots of sharp practices. They do a lot of things that are not even their area of expertise and these are not in the best interest of the patient, because, when someone dabbles in an area that is not their speciality, they can easily make a mistake and it is the patient that bears the brunt of the whole thing. (Interview with a non-DP Consultant)

I have seen a doctor, unlike when you go to a government hospital where you have different departments. Even in medicine, you have different units, such as cardiology, endocrinology, haematology, gastroenteritis and nephrology. So, if a patient is having a liver problem, you refer them to gastroenterology, for diabetes you ask him to go and see the endocrinology, if it is arthritis, you say go and see the haematologist. But, in private practice, a doctor who is supposed to be an endocrinologist may be performing a function in a different area they are not trained in. He is trained, but that is not their expertise. (Interview with a DP Resident)

Clinical decisions are usually taking alone by the dual physician. Dual physicians are jack-ofall-trades, who dabble in areas they are not trained in for financial reasons, and so 
compromise the quality of care.

Medical malpractice and negligence were said to be common with private practice. According to the respondents, some dual practitioners do not adhere to the guidelines for medical practice in the private sector. They may cut corners by ignoring the Standard Operating Procedures (SOPs) since what they do is not subject to the scrutiny by other doctors as found in the public system.

In private practice a lot of things happen. In Nigeria, people are not aware of their rights as they are in the western world. If you do one or two things [medical malpractice] in the private sector, you are not going to be held accountable for that-you can go scot-free. Unlike the public sector, no government will want its hospital to be shut down because of one simple mistake, but, in the private sector, if anything happens the doctor may cover things up and just tell you one or two things, because it is only between the two of you and nobody else knows what happened. In public hospitals, you have your colleagues and they probably watch what you are doing, but, in the private sector, it is not really like that and you can cover up your mistakes and nobody is there to watch what you are doing. (Interview with a non-DP GP)

I am telling you from my own experience that the operating technique in the private sector is different from that of the public sector. If a patient has diabetes, there are guidelines for seeing such a patient in the public sector. But, in the private sector, you might overlook those guidelines because nobody is watching you. In the public sector, whatever you are documenting, the nurse is aware of that and other doctors are aware, but, in the private sector, it is only you. You can even write just two lines that the patient came with headache and vomiting. There may be no details of their history, but that is not.... you might be doing it and succeeding, at the end of day ...... in Nigeria we do not track patients, that is why, sometimes, we think they do well in the private sector. But they don't actually do well. (Interview with a non-DP Resident)

Medical malpractice and negligence are among the common consequences of private practice. The dual practitioner may ignore SOPs and that might put the patient at risk and also negates the principle of a patient-centred approach, which puts the patient above every other consideration. Solo practice by DPs may be riskier to patients than what is practised in the public sector where doctors work in teams with competent colleagues. 


\subsection{Policymakers'/stakeholders' perspectives on disbenefits of DP}

This section outlines disbenefits under three subthemes: dis-benefit to patients, dis-benefit to the public system, and policy makers' perspectives on the disbenefits of dual practice. Each of these subthemes has further supplementary strands.

\subsubsection{Disbenefits to patients}

In this subtheme, a number of aspects were mentioned. These are the high cost of care in the private sector; patient losses due to poor time management by dual practitioners and service denial. Other sub-themes include sharp practice in the private sector and suboptimal quality of service. Each of these themes is discussed below.

\subsubsection{High cost of care in the private sector}

Informants suggested that patients moved from the public system to private facilities then face higher treatment costs. Dual-practice doctors, in their view, would justify this by making patients believe that more expensive care is better quality care.

Those who are engaged in dual practice move patients from the public hospital to their private set-up. By so doing they are diminishing utilization of the public sector through that action. To the patient you are also increasing the cost of care. (Chair, Committee on Health)

I will be worried also that people are paying exorbitantly for the care they should have got at a reasonable cost from the public sector. For me these are the disadvantages of dual practice. (Chair, Committee on Health).

The cost of care is increased when patients are moved to the private sector. The cost in the private sector may be catastrophic for some patients and could drag them below the poverty line. The important issue, however, is that high cost may not be equated to quality of care. Although there is a higher cost in the private sector, the quality of care may not be optimal for all private patients. 


\subsubsection{Denying services to public patient}

Respondents argued against service denial for patients caused by dual practice. They held the view that a service gap is created due to doctors' simultaneous engagement in two work places. They argued that public patients wait longer in the public system due to the doctor's absence, which denies health care services to patients. Selected interview extracts illustrating this follow.

There is no doubt that many people work in two places - both in the government hospital and in the private sector. This has created a lot of service gaps. People [public patients] are not getting what they should get, and government is not happy about it (Director, $\mathrm{MOH})$.

We do not want a situation where a doctor will have one leg in and one leg out, patients will sufferfor it. (Senior Administrator, Medical Council),

Public patients are denied services in many ways in the public system due to dual practice. The non-availability of doctors at the right time, and long wait-times are two of the ways timely services are denied to patients. Some patients visit the public hospital several times before they see the doctor in-charge of their case. Consequently, some of the cases that would have received timely attention may pile up and create backlogs, and in some cases, death or morbidity may result due to delay in proper assessment. In a situation like this, it may be difficult to quantify both direct and indirect costs to patients.

\subsubsection{Sharp practice in the private sector}

Respondents were of the view that sharp practice is more common in the private sector than the public system. They suggested that those sharp practices often occur when private doctors ignore the Standard Operating Procedures (SOPs) guiding clinical practice. In addition, they hold the view that patients are mismanaged in the private sector. Rather than using a standard instrument receiving regular sterilization, they use cheap and unsuitable one such as the use of cooking stove to sterilise instrument.

But at the private level, the SOPs are many and sometimes not followed; unlike in the public facility, where the SOPs most often are followed but in the private practice, many at times they are not followed ( $80 \%$ of the cases). They want to maximise profit and then will not follow the SOPs. So, patients are mismanaged at 
the private level. When they come in at the public level, they are managed better, and the outcome is usually better (Senior Administrator Medical Council).

Ignoring SOPs in the private sector does not only pose danger to the patient but to medical practice. Given the fact that the private sector is rarely supervised due to logistical challenges, unprofessional behaviours may be common. The private doctor can easily use short cuts to minimise cost and achieve financial gain. It is important that supervision is stepped up in the private sector.

\subsubsection{Suboptimal quality of service}

On the issue of quality of care, respondents were of the view that there is suboptimal quality of care for patients. There is less quality time with patients and poor outcomes due to nonavailability of doctors in the public system. In addition, the number of work hours is reduced due to absences, late reporting for work or early ending of clinic sessions/shifts.

What it means is that the quality of care in the public sector will be diminished because of a lack of promptness in initiation of care, when you have to call the doctor to come from his private practice to see their public patients or when facilities particularly in the rural area are shut down because doctors come on Monday and Wednesday, twice in a week. (Chair, House Committee)

They are all busy in their private facilities and nobody sanctions them for doing that. Then you look at patient outcomes, it is poor because when the doctor is not there, they are not giving their best, and not giving their time, the outcome will be poor, complication and death might arise. In terms of training of personnel, it is poor; outcome in terms of patient care will be poor. (Senior Administrator, Medical Council)

The quality of care a patient receives in the public system may not be satisfactory due to the chipping away of official hospital work hours by private practice. In some cases, doctors may come to work late or close early thereby denying patients the opportunity for adequate time with their doctor. Leaving work early may also result in some patients having to repeat their visit to the hospital, especially when their condition requires treatment from one of a few specialists in the facility. Hospital managements and the Ministry of Health do not so far appear to have achieved effective supervision of the public system.

\subsubsection{Disbenefits to the public system}

Respondents mentioned several disbenefits of dual practice to the public system. The main 
issues mentioned were: absence of doctors from work, the notion of DP as a loss to the public system but gain to the private sector, and lack of commitment from dual practitioners.

Respondents suggested that absences from work were common in the public system. Some said that some doctors only report for work on chosen days. These doctors may only come in on two or three days per week. What happens on other days? The respondents were of the view that they use the rest of the days to do their private work.

...but because of profit maximization they prefer to be in their own private facilities to the detriment of public patients. (Senior Administrator, Medical Council)

Doctors do not actually adhere to this policy; you find circumstances where the doctor goes to his private clinic in the morning, at the time he should be in the government hospital, still attending to his private patients. This is not limited to general duty doctors or residents; in fact, it appears to be worse with consultants. For instance, you look at the secondary health care in Enugu state; you hardly will walk into any hospital and find a full complement of doctors at work. So, what is happening? Truancy in the government sector is attributable to dual practice. (Chair, House Committee)

The consequences of absence from work by doctors engaged in dual practice are obvious. Patients wait for too long in the public hospitals for the doctor who is attending to private patients in his/her private practice. Delays in assessment and treatment may occur in the public system when patients have to visit the hospital repeatedly until they are able to access the doctor. This may indirectly create a private market where doctors are readily available. However, the situation is redeemable if the hospital management sees this as a critical issue that demands urgent attention.

Doctors' absence from their official public work due to private practice can result in decreased quality of care and even death. One respondent recounted some of his experiences from serving on a medical disciplinary committee:

I give you a typical example. I was a member of the disciplinary committee that investigated the death of a pregnant woman who lost her life with her baby due to private practice. Her husband happened to know the then Minister of State for Health who then set up a committee to investigate the issue. During the investigation, we found out that the consultant was not in and even when he was told that his attention was needed at the hospital he did not show up, and after the patient died, he did not show up. He left the care to his medical officer who he 
said he trusted and that he said could handle such a case. After his clinic, he was supposed to be on call that started from 4pm; he only went there and browsed through the patients that were available. This woman was admitted in his clinic and he knows that she has a complex case, and ought to have kept his eyes on her, but he did not but went home. His house officer called him saying that he has this kind of patient and he said; call your senior on call and that he could handle it. He spoke with the senior and asked him to take her to the theatre and do what he needs to do. Of course, things went wrong. The committee sanctioned the consultant and he lost his employment. This is just one case that was not reported to the council but was dealt with at the health facility. As a consultant you are not supposed to be there 24 hours, but you should be close by so that if your attention is needed you report. But he never came because he was so busy making his money in his own private practice. (Senior Administrator, Medical Council)

Negative consequences of doctors' absence in the hospital are many. When hospital management allows dual practice to negatively affect staffing during contracted hours that can result in increased morbidity, and in some cases mortality. From the respondents' accounts many avoidable deaths appear to have occurred because doctors denied patients urgent attention. Truancy among public sector doctors is a source of concern to the government, but weak management in government hospitals makes it difficult to challenge these practices.

The respondents mentioned three ways in which government losses from dual practice had a negative impact: erosion of the workforce, loss of revenue to government facilities (through loss of patient payments), and loss of publicly-funded time.

...DP is denying the public facilities the needed workforce, and the time they [DPs] should be at the public hospitals. Because if you are supposed to be working in the public hospital and patients come and you are not there, probably the patient may wait and go. You are denying government that revenue and the service to the people of the state. Therefore, that is not good for the government. There is no way the public health system will benefit from that. Rather they are losing. It is only the DPs that are enjoying themselves, making their money while people are suffering (Director, $\mathrm{MOH}$ ).

What I can see is how dual practice negatively affects the public sector. Those who are engaged in dual practice move patients from the public hospital to their private establishments. By so doing, they are diminishing utilization of the public sector through that action. (Chair, House Committee)

DP deprives government of publicly-funded hours due to absence and truancy. It also loses 
revenue when public patients, who are required to make modest payments for public care, are diverted to private practice. Moreover, an increase in the number of government doctors involved in dual practice will increase the loss to the public sector. If all competent doctors were engaged in dual practice, this would pose a serious threat to the public system.

According to the respondents, a lack of commitment to public service was reflected both in the attitude shown by DP doctors when on duty in public facilities, and the greater attention and commitment they give to their private work.

There was a perception that the "mentality" shown in official duties in the public hospitals was not the same as that evident in private practice. DP doctors earn a full salary in public work but spend fewer hours doing the work and regard patients in a different light.

The attitude of the professional in private practice that same mentality is not applied to the government work and because of that public sector is shortchanged, people assumed that whether they work or not their salaries are certain from the government every month. I mean, in five days they could come twice, stay for two hours and move on to their private practice, after all, nobody notices them except if caught (Senior Official 2, State Health Board).

The respondents suggested that dual practitioners gave more attention to their private practice than they did for the official public work. When doctors engage in dual practice their focus can easily shift to private work, especially if the rules are not well enforced.

One of the disadvantages I know is that government employs and pays doctors to work in the public sector, but their attention is taken over by some of those private interests. In fact, it is one of the allegations levelled against our colleagues that they leave their primary duties and get involved in private practice. Some people [doctors] have their attentions taken over by dual practice and forget what their primary responsibilities are. (Executive Officer 1, Nigeria Medical Association)

If you go around town you see a lot of government doctors who have very big private hospitals, and the law says that a doctor practicing in a public facility should not own or manage a hospital, at least you can manage a clinic where you will have someone who will be there attending to the patient in your absence. You can only go to your own private clinic at the close of work, which is after 4pm. If you as a doctor, fully employed in the public sector and you have a hospital that will take away your time and attention because in your hospital you have to admit patients overnight and you have to be responsible for their management. (Senior Administrator, Medical Council)

From the perspective of respondents, private work seems to gain more attention from 
government doctors than the government work. This kind of work behaviour poses a threat to efficient service delivery in the public system and suggests that the public service ethic of doctors takes second place to the desire for financial gain. This is an evidence of conflict of interest between public and private work. Overall, poor supervision in the public sector may have contributed to sustaining the government work attitude.

\subsection{Chapter summary/conclusion}

The self-rated financial impact of the diverted patients did show both moderate and serious financial impact following payment for private treatment. The payment coping mechanisms for the referred patient group were mainly own money and borrowing. Considering the value of hours lost to the public system of dual practice, the estimated value of hours lost to absences for the various weekly hour (normal routine hour) loss scenarios used in the sensitivity analysis show that five-hour loss to absences means that the value of hours lost to the public system would be N4366579 or USD14270 per year assuming 90\% of the doctors on different grade levels in the hospital engage in dual practice. It is also estimated that if the number of doctors engaged in DP increased to $100 \%$, the value of hours lost to government at 15-hour weekly loss to absences would be N14555263 or USD47566 per year. The estimation was done for the rest of the weekly hour loss scenarios with their computed value of hours lost on both the normal routine and on-call hours.

From the service users' point of view, the disbenefits of dual practice include loss to the public system in terms of revenue loss, extra cost to patients, low commitment of DPs to both the public system and public patients. High rates of absences and late reporting to work were some of the disbenefits indicated by the respondents. The doctors' respondents' perspective indicated how DP affects the public system and drives up cost of care for patients. Patients pay higher in private practice than they would do in a public hospital. Medical training was said to have been affected due to the non-availability of trainers in government hospitals, and this probably limited the capability of public hospitals to undertake certain procedures in a timely manner. 
Similar patterns of responses were found with policymakers'/stakeholders' interviews. According to them, DP drives up cost for patients, and there is a suboptimal care in the public system due to DP. There is conflict of interest with more commitment to private practice than government work. Therefore, the costs to the public system of DP are enormous and may need policy intervention. 


\section{Referral patterns by public sector providers}

\subsection{Extent of physician self-referral}

\subsection{Introduction}

This chapter presents respondents' socio-demographic characteristics, socio-economic status of patients referred, the age group of patients referred from the public system, and the proportion of male and female referred to the private sector. Other findings included in this chapter are providers' referral patterns. The different perspectives of multi-level stakeholders on self-referral from the public system are shown. The views of service users, policymakers/stakeholders and public-sector doctors on self-referral of public patients to private practice are presented, and then followed by a summary and conclusion.

\subsection{Respondents' socio-economic characteristics}

\subsubsection{Socio-demographic characteristics of respondents}


Table 14: Number of referrals and non-referrals and their Socio-demographic characteristics

\begin{tabular}{|c|c|c|c|c|}
\hline Variables & $\begin{array}{c}\text { Referred } \\
n=34\end{array}$ & $\begin{array}{c}\text { Non-Referred } \\
n=373=\end{array}$ & $\begin{array}{l}\text { Total } \\
\mathrm{N}=407 \\
\end{array}$ & F(P-value) \\
\hline Referrals & & & $34(8.4 \%)$ & \multirow{4}{*}{$\begin{array}{l}111.0569 \\
(0.0602)\end{array}$} \\
\hline Non-referrals & & & $373(91.6 \%)$ & \\
\hline \multicolumn{5}{|l|}{ Sex } \\
\hline Male & $18(14.0)$ & $111(86.0)$ & $129(100.0)$ & \\
\hline Female & $16(5.8)$ & $262(94.2)$ & $278(100.0)$ & \\
\hline Age group & & & & 0.9395 (0.5099) \\
\hline $18-38$ & $22(9.1)$ & 221(90.9) & $243(100.0)$ & \multirow{26}{*}{$\begin{array}{l}\text { Mean=38.72 } \\
\text { Range }=19-86 \\
\text { Standard error }= \\
2.347361\end{array}$} \\
\hline $39-59$ & $8(6.1)$ & 123(93.9) & $131(100.0)$ & \\
\hline $60-80$ & $4(12.9)$ & $27(87.1)$ & $31(100.0)$ & \\
\hline 80> & $0(0)$ & $2(100.0)$ & $2(100.0)$ & \\
\hline \multicolumn{4}{|l|}{ Highest Edu level } & \\
\hline Primary school & $3(7.3)$ & $38(92.7)$ & $41(100.0)$ & \\
\hline Junior secondary & $0(0.0)$ & $7(100.0)$ & $7(100.0)$ & \\
\hline Senior secondary & $10(5.8)$ & $161(94.2)$ & $171(100.0)$ & \\
\hline Ordinary National Dip & $4(11.8)$ & $30(88.2)$ & $34(100.0)$ & \\
\hline Higher National Dip & $8(15.7)$ & $43(84.3)$ & $51(100.0)$ & \\
\hline BSc degree & $9(12.7)$ & $62(87.3)$ & $71(10.0)$ & \\
\hline MSc degree & $0(0.0)$ & $10(100.0)$ & $10(100.0)$ & \\
\hline Other & $0(0.0)$ & $16(100.0)$ & $16(100.0)$ & \\
\hline \multicolumn{4}{|l|}{ Marital status } & \\
\hline Currently married & $23(7.3)$ & 293(92.7) & $316(100.0)$ & \\
\hline Single & $9(13.2)$ & $59(86.8)$ & $68(100.0)$ & \\
\hline Separated & $0(0.0)$ & $2(100.0)$ & $2(100.0)$ & \\
\hline Widowed & $2(100.0)$ & $19(90.5)$ & $21(100.0)$ & \\
\hline \multicolumn{4}{|l|}{ Occupation } & \\
\hline Govt worker & $0(0.0)$ & $30(100.0)$ & $30(100.0)$ & \\
\hline Employed in private sector & $5(10.4)$ & 43(89.6) & $48(100.0)$ & \\
\hline Self-employed & $16(8.4)$ & $175(91.6)$ & $191(100.0)$ & \\
\hline Artisan & $8(11.0)$ & $65(89.0)$ & $73(100.0)$ & \\
\hline Student & $0(0.0)$ & $12(100.0)$ & $12(100.0)$ & \\
\hline Unemployed & $2(5.6)$ & $34(94.4)$ & $36(100.0)$ & \\
\hline Other & $3(17.6)$ & $14(82.4)$ & $17(100.0)$ & \\
\hline
\end{tabular}

Table 14 shows that out of the 407 respondents only 34 (8.4\%) were referred from the public system to private facilities. This included 18 referred out of 129 males (14\% of males), and 16 referred of 278 females (5.8\% of females). In terms of the ages of respondents referred, 22 of $243(9.1 \%)$ were referred from the 18-38 age group, while 8 of $131(6.1 \%)$ were referred from the 39-59 age group, and 4 of 31 (12.9\%) were referred from the 60-80 age group. Regarding the educational level of respondents, 9 of 71 (12.7\%) of those in the highest category who held a university degree were referred, and 8 of $51(15.7 \%)$ holding a Higher National 
Diploma were referred. This compares with 10 of 171 (5.8\%) of referrals who completed secondary education, and 4 of $34(11.8 \%)$ that had the Ordinary National Diploma.

Table 15: Comparing NDHS and HH survey data

\begin{tabular}{lll}
\hline Highest Educational Level Area & NDHS (Enugu State) & HHS (Enugu Urban) \\
& $\mathbf{\%} \mathbf{N}$ & $\mathbf{\%}$ \\
Primary & $25.01(61)$ & $10.22(41)$ \\
Secondary & $59.08(318)$ & $44.38(178)$ \\
Post-Secondary & $11.09(751)$ & $41.39(166)$ \\
Other (includes no education) & $4.79(61)$ & $3.99(16)$ \\
Totals & $100.0(1271)$ & $100.0(401)$ \\
\hline
\end{tabular}

Notes. Some categories in the original surveys have been combined to make the results comparable. In the thesis HHS only 401 of 407 respondents answered the education question. The published NDHS survey results for male and female respondents have been aggregated.

The present study is essentially a case study of DP in an urban area in one Nigerian State, Enugu, and therefore subject to the common limitation found in many case studies, that representativeness is difficult to assess. A question arises, however, about how far the sample selected for the study is representative of the population in Enugu State, particularly with regard to socio-economic status. To assess this, demographic data from the thesis household survey was compared with the 2013 Nigeria Demographic and Health Survey (NDHS) (National Population Commission, 2014) data on Enugu State. The one area where comparable data were available related to 'highest educational level', which may be considered to be a proxy for socio-economic status. Data from both surveys showing numbers progressing to primary education, secondary education, post-secondary education, and 'other' (including no education) are shown in Table 15 above. This shows that the Enugu Urban area sample used in the thesis is on average better educated than the Enugu State population as a whole, particularly regarding numbers progressing to post-secondary education. While further investigation would be needed to clarify the reasons for this difference, the author believes it can be attributed to the fact that the NDHS data relates to both urban and rural dwellers, and that rural dwellers typically have significantly lower levels of educational attainment than the 
urban population in the thesis sample. It might be hypothesised that rural dwellers also make less use of DP, although the study did not provide data to confirm this.

\subsubsection{Socio-economic status of patients referred}

Table 16: Number and percentage of respondents in different SES groups referred from the public system

The poorest The very poor The poor The least poor Total $\mathrm{F}(\mathrm{P}$-value $)$

\begin{tabular}{lccccc}
\hline Referred & $2(2.0)$ & $9(8.8)$ & $13(12.7)$ & $10(9.9)$ & $34(8.4)$ \\
$\begin{array}{l}\text { Not } \\
\text { Referred }\end{array}$ & $100(98.0)$ & $93(91.2)$ & $89(87.3)$ & $91(90.1)$ & $373(91.6)$ \\
Total & $102(100)$ & $102(100)$ & $102(100)$ & $101(100)$ & $407(100)$ \\
& & & & & $4.5533(0.2790)$
\end{tabular}

In Table 16, the poorest SES group was just only 2(2.0\%) and the very poor group was 9 $(8.8 \%)$. The poor were $13(12.7 \%)$, and the least poor were10 (9.9\%).

\subsubsection{Age groups of patients referred from public system to private sector}

This section shows the various age groups of patients who were diverted from the public system to the private sector. The number of age groups involved was 18 to 38 years, 39 to 59 years, 60 to 80 years and 80 years and above.

Figure 7: Age group of patients referred from the public system 


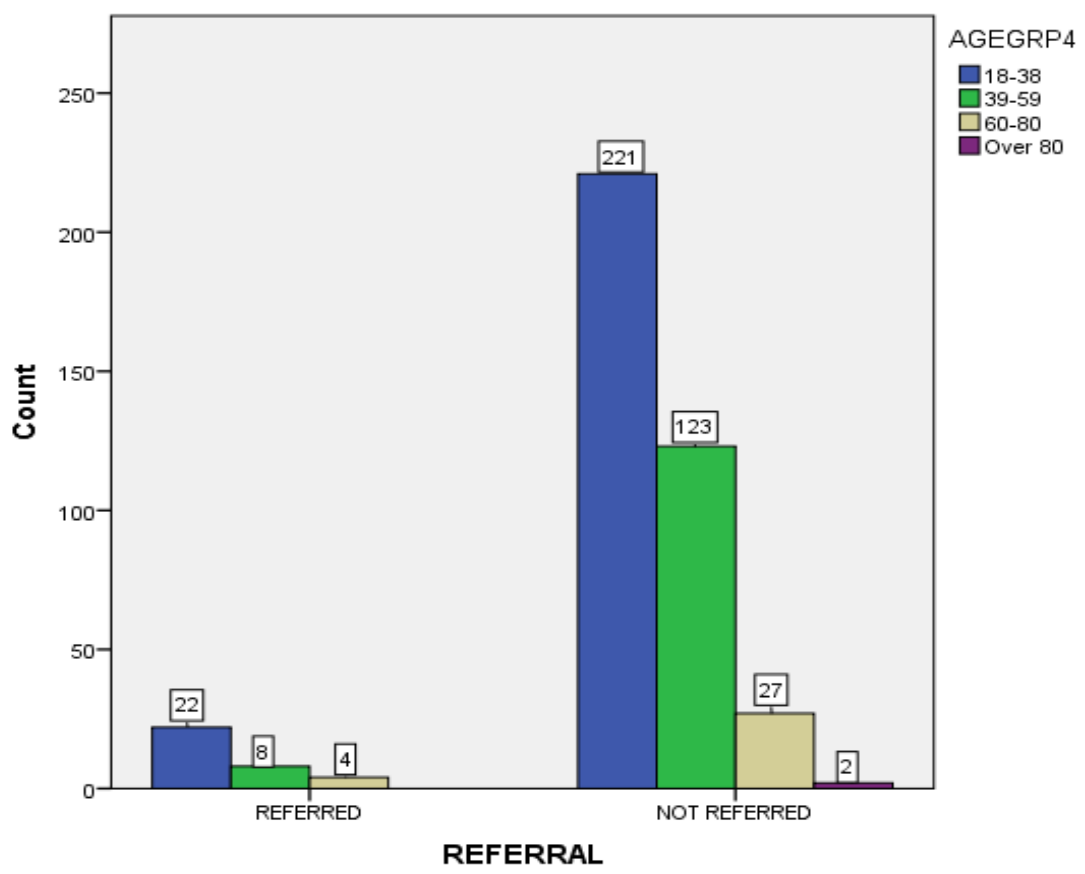

In Figure 7 above, there were more referrals in the age group 18-38 (22) than other age groups. Only 8 patients were referred in the age group 39-59, and 4 in the age group 60-80. Those over 80 years were not referred.

\subsubsection{Proportion of male and female patients referred from public to private hospitals}

In this section, the proportion of male and female who were referred from the public system is shown.

Figure 8: Proportion of male and female patients referred from the public system 


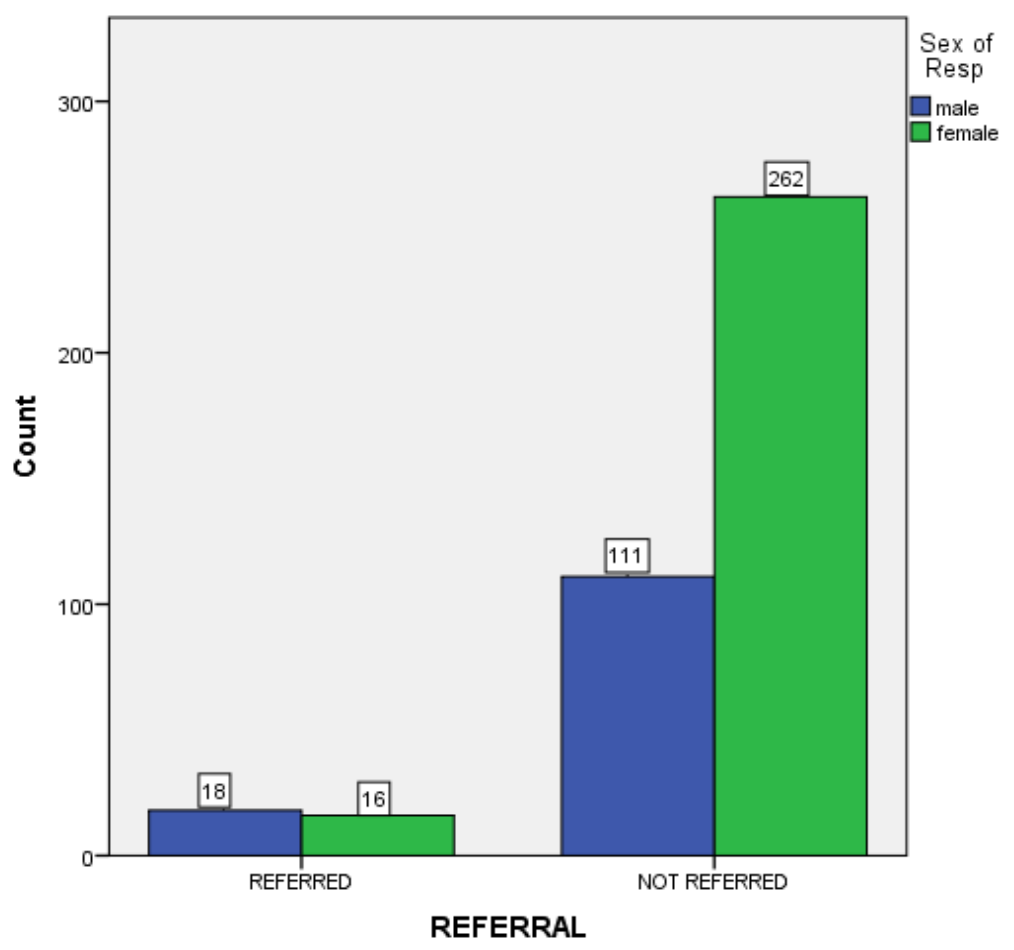

In Figure 8, more male than female were referred from the public system. There were 18 male and 16 female patients respectively who were referred to the private sector.

\subsubsection{Referral patterns used by public sector providers}

This section shows the various patterns used by healthcare providers for patient diversion 
Figure 9: Strategies used by healthcare providers to refer patients from the public system

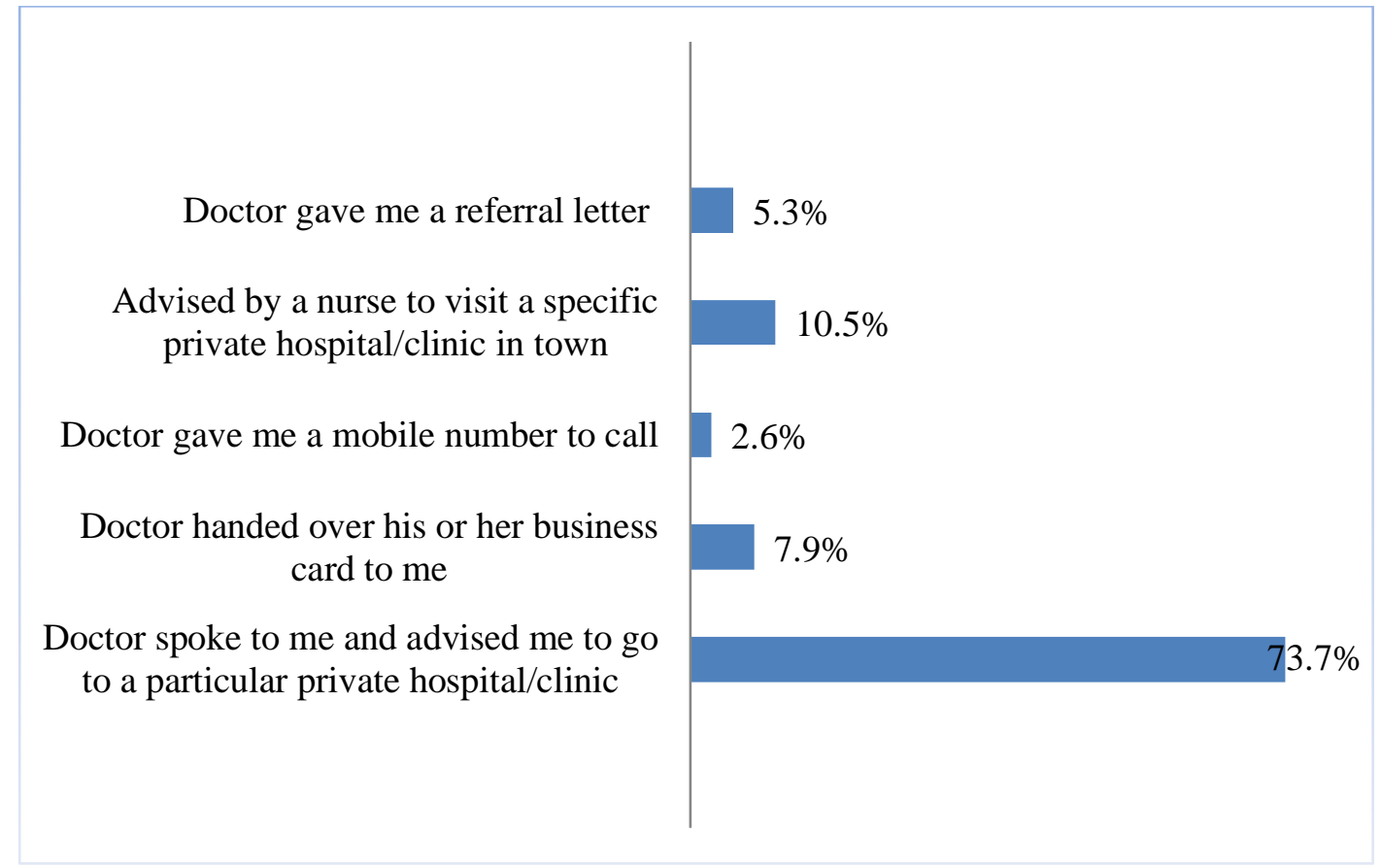

Figure 9 shows that $73.7 \%$ of public patients were advised by doctors to visit a specific private facility whereas those who were advised by nurses to visit some private clinics in town were $10.5 \%$. Only $5.3 \%$ received referral letters from the doctor to go to the private sector.

The quantitative data above show the extent of self-referral by public sector providers by examining the number of referrals in the population, which was estimated at $8.4 \%$. Age group 60-80 was mostly referred to the private sector, whereas a higher proportion of male than female was self-referred from the public sector representing $14.0 \%$ and $5.8 \%$ respectively. The referral patterns used by public sector providers, show that there could be direct and indirect self-referral of patients to the private sector.

\subsection{Perspectives on self-referral}

This section covers qualitative data collected from different categories of respondents. It includes the perspectives of service users, doctors and policy makers/stakeholders on referral of public patients to the private sector. This helped us to draw a comparison and to understand 
the stance of respondents on self-referral of patients in the public sector and what their views might mean for the health sector and service delivery in the public system.

\subsection{Service users' perspectives on self-referral}

It was found that the majority of the participants had had the experience of self-referral in the past 12 months. The dual practitioner self-refers patients to their private practice, or that of their colleagues or use 'agents' such as nurses or other healthcare workers to divert patients to their private clinics. In this instance, patients do not receive services that would have been delivered at no or low cost in the public sector and are now subject to payment. Some of them were self-referred by doctors and others by nurses working in the same hospital.

Some of the experiences are narrated below.

Concerning what is being discussed, I have been to a government hospital, and they referred me to a private hospital owned by a doctor working in the same government hospital. I stayed the whole day in the government hospital and left without been given attention and came back the following day. When I came the following day, I spoke with one Rev X, a doctor, who also works in that government hospital. She said that my case would not be treated in that government hospital, and that she will direct me to one of their doctors who is a gynaecologist here in this hospital. I left the hospital [government] to the private hospital where she directed me. When I reached there the doctor was not there but one of the nurses called him on phone immediately and explained my condition to the man. And within ten minutes he was called, the doctor rushed to his private hospital and opened a file for me without delay. He called his son to take me to where I did the laboratory test and treatment was commenced immediately and within 6 hours I started recovering. But when I went to the government hospital they said eeeeehi. I have never seen this kind of condition since my 21 years of medical practice [all laughed, participants making mockery of the doctor's reaction to the case] or maybe he did not study that case in his medical education. But when I came to the private hospital the doctor commenced work immediately, and before you know it he hung my two legs up and started giving me injections. Although, that baby later died; I still delivered the baby. He treated me until I was ok. I have had two babies after that one, and there was no problem with my womb (FGD 4. P.3)

I had an experience during my first year in the university. I had a severe sickness during this time and my family took me to a tertiary hospital (public). The money I was charged was little but the problem I faced was that of poor service. I spent three days there but did not see the doctor in-charge of my case. He did not come 
to work for these three days. At this time my legs were swollen up. So, my father took me to another government hospital in a neighbouring state. There in that hospital, the same doctor who supposed to have treated me in the first tertiary hospital I was admitted was also working in the second hospital I visited. My brother is a medical doctor and he insisted that only that doctor will treat me. What the doctor did was to refer me to where I could take a laboratory test, and after the doctor had seen the test result, he suggested that I should be discharged and sent back to Enugu. When I came down to Enugu, I was admitted at one private hospital at $X$ road and to my greatest surprise that same doctor owns the private hospital. The services of public hospital are affordable, but patients are not receiving good services (FGD2. P.1).

Patients pay extra in the private sector when they are diverted from the public sector. Some have argued that dual practice gives patients access to consultants who might not be readily available in the public sector. Though, self-referral might be in the interest of patients, but often, that may not be the case, as it increases their financial burden. The practice of diverting patients from the public system may suggest that financial interest as against professional altruism seems to influence referral of patients from the public system. Overall, the perception that seems to be an indictment on the public system is that it appears to have failed to protect public patients from the overbearing power of the professionals by failing to introduce stringent measures against self-referral.

\section{Doctors' perspectives on self-referral}

This section deals with respondents' views on patient diversion. It is shown that dual practitioners divert patients from the public to the private sector. The discussion on how diversion takes place reveals that doctors can use nurses to divert patients to their clinics (indirect diversion) or using the tactics of ignoring patients or being rude to them as a way of making them consider private treatment as an alternative.

\section{Direct diversion}

The respondents narrated their various experiences about patient diversion. One common scenario is that doctors directly tell patients to go to their private clinics and wait for them.

It is like drawing away patients from the public health facility to the private. It is like that. For me, my conscience would not allow me to do that and more so 
because of my personality. It is insulting to realise that somebody can ask someone, especially in a public health facility, to follow him or her to his or her private set up. Of course, it happens in Nigeria. I wouldn't say that I can name an example, but I know it happens, people telling patients from the public facility to go there and wait for them [i.e. in their private clinics]. (Interview with a DP GP)

The circular that was sent to this place is not about the prohibition of DP, but it indicates that government is frowning on it. The situation is bad because medical doctors know the ethics but continue to siphon patients from this health facility to their private hospitals. They also have people there working for them. So, work is also going on in their own hospitals. But the idea of moving them from the public hospital is the criminal part of it. (IDI with a non-DP Consultant)

Some doctors frown at this behaviour and see it as unprofessional. This behaviour may deprive government hospitals of revenue but conversely help the patient to receive timely treatment. It looks like a double-edged sword and raises the question of whether the diverted patient is satisfied or feels 'ripped-off' following the private treatment received.

One non-DP respondent argued that self-referral targets less-serious cases. He argued that dual physicians cherry-pick patients who do not have life-threatening cases. That means that simple cases might be diverted to private practice, leaving those with more serious conditions in the public sector.

Most times before someone decides to come to the teaching hospital it's usually because of a very serious health condition and most of them will be very bad cases that the dual practitioner might not want to see in their private hospitals, because it is going to cost them money and they may not gain financially from the patient, especially chronic cases. (Interview with a non-DP Snr. Registrar)

\subsubsection{Diversion based on patient welfare}

Some of the respondents contended that some referrals are made for clinical reasons and therefore are motivated by a desire to guarantee patient safety and good management.

Yes, sometimes a public hospital might not offer some services because there are some specialities that are not well-developed in the public sector but are welldeveloped in the private sector. In that case, they can refer to the private sector. There are different specialities, like neurosurgery, ophthalmology and some aspects of Obs and Gynae, especially IVF [in vitro fertilisation]. Some private hospitals do it very well, but, if there is no such speciality in the public sector, they will refer to the private sector where the speciality is better developed. Or, 
some investigations that are not available in the public sector can be referred to the private sector to handle those investigations which may require a CT scan, or MRI; those investigations can actually be referred from the public sector to the private sector (Interview with a DP Consultant).

Where a patient needs a specialist service, doctors can use their discretion to refer the patient to a hospital with the necessary facilities. But it can sometimes be difficult to disentangle patient welfare motives from financial motives if the referral is made to hospitals or clinics owned by friends of the referrer.

\subsubsection{Indirect diversion}

Indirect diversion was said to mainly take two forms. First, there is the use of nurses to help doctors to divert patients to their private clinics. Second, some dual physicians can make the public system difficult for patients to access as a tactic to push them towards their private practices.

Doctors can refer, or they can do that through the nurses. For example, sometimes, after seeing the doctor, they may ask you to come back in three days' time and the nurse will ask if you asked the doctor for their contact number. And, if you did not, the nurse will say, 'are you a fool? You may not see him at the hospital in the next appointment; you know he is a consultant, go and ask him to tell you his private hospital.' The nurse might give you the consultant's number, so that it does not look as if it is the doctor that diverted you to his private hospital. When the doctor sees you, he may ask, 'what are you doing in my hospital?' Then, the patient will say 'someone directed me to see you here.' So, it is common. But, in the teaching hospital, people are always careful about it. It is more common in general and specialist hospitals where the monitoring is not too serious. (Interview with a non-DP Snr. Registrar)

Because the only way to make patients come to their private clinics is to subtly make it difficult for patients to go to a public hospital, so that way they can drift them to their private hospitals. But, if a doctor consults in other hospitals not owned by him, of course, he is not under much pressure, unlike the pressure he faces when he runs his own private hospital. At the end of the month, whether he sees patients or not, he must pay salaries to his employees, and that may push them to make sure they survive in the market, but if, on the other hand, they are consulting in an established hospital, if they have patients, they go and see them and, if not, they do not lose anything. (Interview with a non-DP Consultant) 
This tactic may seem difficult for patients to detect, especially in a context where healthcare providers are well trusted.

\subsection{Policy makers'/stakeholders' perspectives on the diverting of patients to private care}

When talking about the steering of patients away from the public system, stakeholders talked about two aspects: the use of agents to divert public patients, and how doctors might also be directly involved in the practice.

\subsubsection{Use of agents/middlemen to divert patients}

According to informants, many public doctors use agents to divert patients to their private practice. These agents may be both healthcare and non-healthcare staffs who receive payment for diverting a public-sector patient to the doctor's private facility.

I remembered when I was doing my house job in a general hospital in Lagos State. They posted me to the Obs and Gynae Unit of the hospital. Of course, many of these deliveries and complications will come up at night, and I had my senior medical officer who was deputizing for the chief consultant who was not available that night. Right from the gate of the hospital, you see the gate men scouting for patients to refer to the doctor's private hospital so that they could get some commission. (Senior Administrator, Medical Council)

This approach to scout for patients shows the level of competition in the private sector and how that might not be for patient interest.

\subsubsection{Patient diversion by medical staff}

It was said that doctors themselves often pressure patients to divert to private care. According to respondents they use a variety of pretexts. They may for example dishonestly claim that all the public beds are already taken, and that the urgency of treatment need means the patient must pay to get seen.

There was my senior medical officer who hid under the guise that all available bed spaces are occupied and that they cannot admit a patient on the floor and this patient was an eclamptic patient and an eclamptic patient would have convulsion, high blood pressure and in that condition the mother and the foetus are at risk. 
So, this patient was taken to this senior medical officer's private facility and the patient died on the operating table. (Senior Administrator, Medical Council)

A typical example is when you go to the private Clinic $X$ where $\operatorname{Dr} Y$ and Co were doing private practice. When they were working at the government hospital A at Enugu, the place [the private clinic] was booming, but when they retired, their source of patient supply [government hospital] dried up. When they were working there, the place was booming. You would never see them at government Hospital $C$; they were busy diverting patients from Hospital $C$ to their private clinic, but when they retired from Hospital $C$ there was no more source of diversion. This is a typical case study that when you study them you will then be laughing. If you go there now, it is a shadow of itself. (Senior Administrator, MoH).

Some of the doctors justify diverting patients by mentioning the poor conditions of public hospitals.

Some senior respondents made their disapproval of diverting patients clear, saying that it reduced the utilisation of the public hospital and deprived it of patient income.

Those who are engaged in dual practice move patients from the public hospital to their private clinics. By so doing, they are diminishing utilization of the public sector through that action (Chair, House Committee)

But by and large it is depleting government resources and subsidizing the private sector. To the practitioner, I think most of them will end up losing. They use the little salary they earn to maintain those facilities, that is why most of them have closed up shop. (Senior Administrator, $\mathrm{MOH}$ )

It was explained that sanctions were meted out to culprits whose behaviour was discovered.

And because that patient had a relation who was very influential in government that matter was brought up and was not swept under the carpet. The medical officer was suspended at the end of the day and his employer terminated his appointment. The consultant who was supposed to be in-charge, who was not available in the public hospital, was also sanctioned, I think he was suspended for some period and was later recalled. (Senior Administrator, Medical Council)

Respondents claimed that patient diversion was common among doctors who engaged in dual practice. Although doctors can often find reasons to justify it, the senior stakeholders interviewed generally took a negative view of the practice. They suggested that diversion not 
only robs public hospitals of revenue but also increases the cost of care to patients and sometimes results in negative outcomes.

\subsection{Chapter summary/conclusion}

Chapter 6 examines the patterns of referral by DPs working in the public sector. The diversion of the SES groups shows that the poor group was mostly diverted with 13 (12.7\%), followed by the least poor with $10(9.9 \%)$, and the very poor, which had $9(8.8 \%)$ diversions. The least diverted group was the poorest with 2 or $(2.0 \%)$ whilst patients aged $60-80$ has the highest proportion of referrals and they are more likely to be referred. Conversely, the least diverted age group was those aged 39-59. Males were more likely to be diverted than females. Overall, females, the poorest SES group, and the middle-aged group are least likely to face diversion.

The qualitative data confirms the practice of self-referral in the public hospitals. The data from the three categories of the respondents - the service users, the public-sector doctors, and the policymakers/stakeholders - confirm the commonality in the diversion of public hospital patients to private practices. Patient diversion could occur directly with the DP self-referring a patient to his/her own private clinic, or indirectly when nurses or other healthcare workers were used as agents to send patients from government hospitals to the DPs' private clinics.

This evidence is highly relevant to the debate on the financial self-interest of medical professionals and raises questions about the future of public healthcare in Nigeria and what should be the role of the state. 


\section{Are there differences in quality of healthcare in public system and private practice managed by dual practice physicians?}

\subsection{Comparing differences in quality of healthcare in public system and private practice managed by dual practitioners}

This chapter presents the findings on differences in quality of healthcare in the public system and private practice of dual practice physicians. The chapter is structured in two sections. First section deals with the results from the descriptive Likert analysis of the survey questionnaire that compares differences in quality of healthcare between public and private practices of the same doctor using the Donabedian healthcare quality model. The second section presents findings from service users who compared services in both sectors based on their hospital visits experiences and according to Donabedian's three elements of healthcare quality - structure, process and outcome.

\subsection{Descriptive Likert analysis of service users' experience of healthcare quality in public system and private practice managed by dual practice physicians}

This section presents the Likert analysis that compares how respondents viewed healthcare quality of public and private practices of the same doctor using the Donabedian model, which looks at quality healthcare in terms of structure, process and outcome. 
Table 17: Comparison of structure element of healthcare quality between DPs' private practice and public practice $(N=407)$

\begin{tabular}{|c|c|c|c|c|c|c|c|}
\hline Question & $\begin{array}{l}\text { Strongly } \\
\text { disagree } \\
\mathbf{n}(\%)\end{array}$ & $\begin{array}{l}\text { Disagree } \\
\mathbf{n}(\%)\end{array}$ & $\begin{array}{l}\text { Don't } \\
\text { know } \\
\text { n(\%) }\end{array}$ & $\begin{array}{l}\text { Agree } \\
\mathbf{n}(\%)\end{array}$ & $\begin{array}{l}\text { Strongly } \\
\text { agree } \\
\text { n(\%) }\end{array}$ & $\begin{array}{l}\text { Mean } \\
\text { [SD] }\end{array}$ & $\begin{array}{l}95 \% \\
\text { CI } \\
\text { (lower- } \\
\text { upper) }\end{array}$ \\
\hline $\begin{array}{l}\text { 34a). Better physical } \\
\text { infrastructure (buildings) exist } \\
\text { in public hospitals than in } \\
\text { private practices set up by } \\
\text { government doctors }\end{array}$ & $2(0.5)$ & $18(4.4)$ & $2(0.5)$ & $157(38.6)$ & $228(56.0)$ & $4.5(0.8)$ & $4.4-4.5$ \\
\hline $\begin{array}{l}34 \mathrm{~b}) \text { There is better } \\
\text { availability of equipment in } \\
\text { public hospitals than in private } \\
\text { practices of government } \\
\text { doctors }\end{array}$ & $15(3.7)$ & $46(11.3)$ & $3(0.7)$ & $178(43.7)$ & $165(40.5)$ & $4.1(1.1)$ & $4.0-4.2$ \\
\hline $\begin{array}{l}34 \mathrm{c}) \text {. There are fewer } \\
\text { qualified health care staff in } \\
\text { private practices of } \\
\text { government doctors compared } \\
\text { with public facilities }\end{array}$ & $26(6.4)$ & $55(13.5)$ & $9(2.2)$ & $174(42.8)$ & $143(35.1)$ & $3.9(1.2)$ & $3.7-4.0$ \\
\hline $\begin{array}{l}34 \mathrm{~d}) \text {. Government doctors } \\
\text { engaged in private practice } \\
\text { have better opening and } \\
\text { closing hours than public } \\
\text { hospitals }\end{array}$ & $15(3.7)$ & $73(18.0)$ & $26(6.4)$ & $172(42.5)$ & $119(29.4)$ & $3.8(1.2)$ & $3.7-3.9$ \\
\hline $\begin{array}{l}34 \mathrm{e}) \text { There is better } \\
\text { availability of drugs in private } \\
\text { practice of government } \\
\text { doctors than in government } \\
\text { hospitals }\end{array}$ & $112(27.8)$ & $127(31.5)$ & $23(5.7)$ & $81(20.1)$ & $60(14.9)$ & $2.6(1.5)$ & $2.5-2.8$ \\
\hline
\end{tabular}

Scores ranged between 1 and 5

The above Table presents a simple comparison of the structure element of healthcare quality between public and private practices of the same doctor. It examines differences in perceived care quality in both practice sites used by the same doctor in relation to the physical attributes of care. By summing the views of respondents who 'agreed' and 'strongly agreed' on the question items on structure element we can see that an overwhelming majority (94.6\%) of respondents believed that government hospitals have better physical infrastructure (buildings) (Item 34a). A similar trend was observed in equipment availability where $84.2 \%$ reported much better availability of equipment in the public system (Item 34b). Drug availability was another area where over half of the respondents $59.3 \%$ hold the view that the public system is 
doing better (Item 34e). Comparing the number of qualified healthcare personnel that deliver services in both practice sites shows there were seen to be fewer qualified healthcare staff in private practice with $77.9 \%$ agreeing (Item 34c). The reason for this might be the high cost of keeping qualified personnel in private practice. On the issue of business opening and closing times, a clear majority of the respondents believed that private practice has better opening and closing time with $71.9 \%$ of respondents upholding this view (Item 34d). This might be due to the pressure to pay for the cost of running private practices, which may require long business hours to accommodate patients arriving at odd hours.

Better physical infrastructure seems to exist in the public system than in private practice. Building a hospital is a major capital project that, except for a few well-financed private ventures, only the Government can afford. Many doctors mainly use rented houses or flats in apartment blocks to open clinics and hospitals. Similarly, Government is the entity best able to afford the provision of costly medical equipment. This is something that may not be within the reach of many DPs who simply cannot fund the cost and maintenance of the latest hightechnology medical equipment. 
Table 18: Comparison of process element of healthcare quality between DP's private practice and public practice $(N=407)$

\begin{tabular}{|c|c|c|c|c|c|c|c|}
\hline Question & $\begin{array}{l}\text { Strongly } \\
\text { disagree } \\
\text { n(\%) }\end{array}$ & $\begin{array}{l}\text { Disagree } \\
\mathrm{n}(\%)\end{array}$ & $\begin{array}{l}\text { Don't } \\
\text { know } \\
\text { n(\%) }\end{array}$ & $\begin{array}{l}\text { Agree } \\
\text { n(\%) }\end{array}$ & $\begin{array}{l}\text { Strongly } \\
\text { agree } \\
\text { n(\%) }\end{array}$ & $\begin{array}{l}\text { Mean } \\
\text { [SD] }\end{array}$ & $\begin{array}{l}\text { 95\% } \\
\text { CI } \\
\text { (lower- } \\
\text { upper) }\end{array}$ \\
\hline $\begin{array}{l}\text { 34f). The waiting time to } \\
\text { see a doctor in public } \\
\text { hospitals is less than the } \\
\text { waiting time to see him in } \\
\text { his private practice }\end{array}$ & $108(26.5)$ & $178(43.7)$ & $15(3.7)$ & $53(13.0)$ & $53(13.0)$ & $2.4(1.4)$ & $2.3-2.6$ \\
\hline $\begin{array}{l}34 \mathrm{~g} \text {.) There is more time to } \\
\text { discuss my medical } \\
\text { problem with a } \\
\text { government doctor in } \\
\text { public hospital than in } \\
\text { private practice }\end{array}$ & $106(26.0)$ & $220(54.1)$ & $18(4.4)$ & $33(8.1)$ & $30(7.4)$ & $2.2(1.1)$ & $2.1-2.3$ \\
\hline $\begin{array}{l}34 \mathrm{~h}) \text {. Patients are not } \\
\text { treated with courtesy and } \\
\text { respect by government } \\
\text { doctors in public hospitals } \\
\text { compared with private } \\
\text { practices }\end{array}$ & $14(3.5)$ & $76(18.8)$ & $34(8.4)$ & $154(38.0)$ & $127(31.4)$ & $3.7(1.2)$ & $3.6-3.9$ \\
\hline $\begin{array}{l}\text { 34i). Patient confidentiality } \\
\text { is not taken seriously by } \\
\text { government doctors in } \\
\text { public hospitals compared } \\
\text { with private practices }\end{array}$ & $24(5.9)$ & $77(19.0)$ & $58(14.3)$ & $145(35.8)$ & $101(24.9)$ & $3.6(1.2)$ & $3.4-3.7$ \\
\hline $\begin{array}{l}34 \mathrm{j}) \text {. Doctors in public } \\
\text { hospitals take more time to } \\
\text { listen carefully to patients } \\
\text { than in private practices }\end{array}$ & $108(26.6)$ & $213(52.5)$ & $17(4.2)$ & $33(8.1)$ & $35(8.6)$ & $2.2(1.2)$ & $2.1-2.3$ \\
\hline $\begin{array}{l}34 \mathrm{k}) \text {. Government doctors } \\
\text { while in their private } \\
\text { practices take their time to } \\
\text { explain things in a way I } \\
\text { could understand } \\
\text { compared with when they } \\
\text { are in public hospitals }\end{array}$ & $10(2.5)$ & $40(9.9)$ & $6(1.5)$ & $185(45.8)$ & $163(40.3)$ & $4.1(1.0)$ & $4.0-4.2$ \\
\hline $\begin{array}{l}\text { 341). I have a rapport with } \\
\text { my doctor in his private } \\
\text { practice compared with } \\
\text { when I visit him in the } \\
\text { government hospital }\end{array}$ & $8(2.0)$ & $39(9.6)$ & $6(1.5)$ & $157(38.6)$ & $197(48.4)$ & $4.2(1.0)$ & $4.1-4.3$ \\
\hline
\end{tabular}

Scores ranged between 1 and 5

The above Table compares the process element of healthcare quality in DPs' public and private practices. It measures respondents' perceptions of what is done in the process of 
receiving care in both places of practice. Again, the percentages of 'agree' and 'strongly agree' were combined to obtain a simple agreement in each question statement.

Most of the respondents reported having a better rapport with their doctor in private practice with $87 \%$ agreeing (Item 341). Private practices are usually smaller in nature and it may be easier to build rapport with a reduced number of patients. The level of rapport in private medicine could make private doctors go the extra mile in explaining things in a way patient can understand - a perception shared by most respondents with $86.1 \%$ agreeing (Item $34 \mathrm{k}$ ). On the availability of more time to discuss patients' medical problems in government hospitals, only $15.5 \%$ of respondents would agree (item $34 \mathrm{~g}$ ), which suggests that private practice is seen to give more time for patients. Although, confidentiality is a patient right, the public system was seen as likely to compromise this by $60.7 \%$ of respondents (Item $34 \mathrm{i}$ ). Courtesy and respect accorded to patients were perceived as higher in private practice with $59.1 \%$ agreeing (Item $34 \mathrm{~h}$ ), while the perception that there is less wait-time in the public sector was agreed only by $26 \%$ of respondents (Item 34f). Similarly, only $16.7 \%$ of respondents agreed that government doctors take more time to listen carefully to patients in government hospitals (Item 34j).

The above summary shows that rapport with a doctor is seen to be better in private practice which indicates a better relationship between a doctor and private patients. In the view of respondents, this improved relationship could also have a positive effect on how the doctor explains and communicates medical issues to private patients. In the public system, there may be a limited time assigned to each patient, which may not be enough for appropriate medical history taking or explanation of proposed treatments. Although the distribution of responses between 'strongly agree' and 'agree' in 'courtesy and respect' has a higher mean, the combined percentages of the number agreeing was shown to be slightly higher for 'patient confidentiality not taken seriously' in the public system. 
Table 19: Comparison of outcome element of healthcare quality between DPs private practice and public practice $(N=407)$

\begin{tabular}{|c|c|c|c|c|c|c|c|}
\hline Question & $\begin{array}{l}\text { Strongly } \\
\text { disagree } \\
\text { n(\%) }\end{array}$ & $\begin{array}{l}\text { Disagree } \\
\text { n(\%) }\end{array}$ & $\begin{array}{l}\text { Don't } \\
\text { know } \\
\text { n(\%) } \\
\end{array}$ & $\begin{array}{l}\text { Agree } \\
\text { n(\%) }\end{array}$ & $\begin{array}{l}\text { Strongly } \\
\text { agree } \\
\text { n(\%) } \\
\end{array}$ & $\begin{array}{l}\text { Mean } \\
\text { [SD] }\end{array}$ & $\begin{array}{l}\text { 95\% CI } \\
\text { (lower- } \\
\text { upper) }\end{array}$ \\
\hline $\begin{array}{l}34 \mathrm{~m}) \text {. I am more satisfied when } \\
\text { treated in a public hospital by } \\
\text { government doctors than when } \\
\text { treated in their private practices }\end{array}$ & $142(34.9)$ & $141(34.6)$ & $9(2.2)$ & $72(17.7)$ & $43(10.6)$ & $2.3(1.4)$ & $2.2-2.5$ \\
\hline $\begin{array}{l}34 \mathrm{n}) \text {. The nature of my illness } \\
\text { condition and the treatment } \\
\text { options open to me are } \\
\text { explained better by the } \\
\text { government doctor in his } \\
\text { private practice than in the } \\
\text { public hospital }\end{array}$ & $18(4.4)$ & $63(15.5)$ & $8(2.0)$ & $164(40.3)$ & $154(37.8)$ & $3.9(1.2)$ & $3.8-4.0$ \\
\hline $\begin{array}{l}340) \text { The recovery rate is } \\
\text { higher in public hospitals than } \\
\text { in private practice }\end{array}$ & $64(15.7)$ & 161(39.6) & $55(13.5)$ & $86(21.1)$ & $41(10.1)$ & $2.7(1.3)$ & $2.6-2.8$ \\
\hline $\begin{array}{l}34 \mathrm{p}) \text {. My health status } \\
\text { improves more when treated } \\
\text { in public hospital by } \\
\text { government doctors } \\
\text { compared with when treated } \\
\text { in their private practices }\end{array}$ & $79(19.6)$ & $202(50.0)$ & $29(7.2)$ & $58(14.4)$ & $36(8.9)$ & $2.4(1.2)$ & $2.3-2.6$ \\
\hline $\begin{array}{l}34 q) \text {. I would recommend } \\
\text { visiting a government doctor } \\
\text { in his private practice as } \\
\text { opposed to a government } \\
\text { hospital }\end{array}$ & $28(6.9)$ & $49(12.1)$ & $3(0.7)$ & $143(35.2)$ & $183(45.1)$ & $4.0(1.2)$ & $3.9-4.1$ \\
\hline
\end{tabular}

Scores ranged between 1 and 5

Table 19 compares the outcome element of healthcare quality obtained in the two practice sites. It shows the difference in outcome of care between the public system and private practice of public-sector doctors. Once again 'agree' and 'strongly agree' and 'disagree' and 'strongly disagree' have been combined to get the total percentage agreeing with each question item. An overwhelming majority of the respondents would recommend visiting private practice with $80.3 \%$ agreeing (Item $34 q$ ). Willingness to recommend private practice is probably connected with patients' perceptions of satisfactory service. A related factor is the nature of patients' illness conditions and treatment options, which a majority $(78.1 \%)$ of respondents agreed were better explained and communicated in private practice (Item $34 \mathrm{n}$ ). It could be that market incentives drive DPs to give better explanations to patients in the private 
sector. On the issue of recovery rate following treatment received, only $31.2 \%$ of respondents believed that the recovery rate was higher in public hospitals (Item 34o). Likewise, in respect of health status improvement, only $23.3 \%$ believed this was greater when treated in the public system (Item 34P). Surprisingly, a small number of service users (28.3\%) indicated that they were more satisfied when treated in a public hospital (Item $34 \mathrm{~m}$ ).

Many respondents would recommend visiting a government doctor in their private practice as against government hospitals. This seems to be linked to the perception that DP doctors provide more timely attention to private patients. Respondents also felt that the nature of illness conditions and treatment options open to them were explained better in private practice. The responses to the question items seem to suggest that private practice is preferred to the public system in terms of the outcome element of quality.

\subsection{How do respondents perceive healthcare quality?}

In this section, the findings cover service users' perception of healthcare quality. It further presents the comparison of the quality of healthcare in the public system and private practice of the same doctor using the Donabedian healthcare quality model (structure, process and outcome elements of quality). Details on Donabedian's elements of quality are found in (Chapter 3). Quality of healthcare has different meanings for different service users. Each dimension is presented as a sub-theme in the analysis.

\subsection{Respondents' perceptions of healthcare quality}

The study found that immediate attention to a patient by a doctor is regarded as a key indicator of healthcare quality. A lack of equipment is seen as an indicator of poor healthcare quality. It was also noted that when patients are insulted in the process of receiving care, that is a pointer of poor-quality care. Quick recovery from treatment received is also regarded as quality healthcare. 
If I get a quick attention when I come to the hospital, even if I was given a first aid treatment; it gives me a reassurance that I have been cared for and my mind will accept it (FGD1. P.7).

Some nurses have bad manners and use bad words on patients, and it is true especially in government hospitals. If I receive insult in a hospital or in a treatment centre; I do not value such treatment as quality (FGD 2. P.6)

The way they come to me and were asking me how I was feeling, and if I was ok really impressed me. When I went there in my first pregnancy, unfortunately, I had a still birth, but the way they were up and doing, calling phones to seek information; they would run out to buy drugs and gave me injections. The overall care they gave me, in fact, they were humane in their treatment. The nurse will give me injection and she will say sorry. They came to the ward when I was in labour and were singing songs for me. During the push, they gave me palliative support and after the delivery they were chatting me up and saying things that gladdened my heart. (FGD3. p.3)

Every service user may perceive quality of healthcare differently depending on experience. What constitutes a quality healthcare for one patient may not be for another. A treatment that suits one person may not suit the other, even when they have the same health condition. However, patients seem to be more concerned with the process element of quality as that is the aspect that seems most visible and comprehensible to them.

\subsection{Comparing quality of healthcare in public system and private practice using Donabedian model}

A comparison of quality of care is drawn between public and private healthcare managed by dual practitioners to assess the difference in healthcare provided by DPs in their private practice and the public sector. It shows whether dual practitioners offer better quality of care to private patients dissimilar from the public patients. The comparison was structured around the three elements of quality used in this study. Each of them is discussed as follows.

\subsection{Structure element of quality}

One of the three elements of quality covered in the study is the structure element, which defines the physical attributes of the environments where care takes place. This study 
compares the physical element of quality to understand the difference between the public system and private practice of dual physicians. The subcategories that emerged under this sub-theme are drug availability, buildings /equipment, and human resources.

\subsubsection{Availability of drugs}

Some of the participants were convinced that government hospitals have better drugs than those of private practice. Others were of the view that private hospitals and clinics set up by public doctors have more drug availability and are more effective.

With all the equipment and good drugs in the government hospital, if only the doctors can bring in the same determination and vigour they use in their private practice in the government work, I would say that quality of care in government hospital is better than that of private practice (FGD 3, P.5).

But in the government hospital, they would simply prescribe the drugs and ask you to go and buy outside the hospital. If you do not have money to provide those things, they will not attend to you. Like what happened to my sister who went to a government hospital for her child who was said to be lying in the breech position. After she was prescribed some drugs and all the things she had to buy, the husband went out to look for money to purchase all the drugs, while he was running about looking for the money the child died in the womb ... (FGD 1, P.5)

Though these private hospitals are costly, some of them have more effective drugs than the government's own. The reason for having quality drugs in their private hospitals and clinics is to enable you to come there. So, these dual practitioners know what they are doing. They keep the best of drugs to make people come to their own hospitals and clinics (FGD3, P.4).

There seems to be more drug availability in the private practice than the public, though some participants strongly held the opinion that drug availability is more in doctor's private practice. The drug supply system differs significantly from that of the private sector. Drug availability in the public system depends on a number of factors. Drug supply chain in the public system may require some bureaucratic processes that might delay supply over a given period of time. There might be some bottlenecks at the central medical store from where drugs are sent on request to the state. Those administrative hiccups cannot be ruled out in drug supply and availability in the public system. Again, in terms of prescription of drugs in the public system, doctors adhere to the recommended essential drug list, and then the bigger 
issue of poor funding of the public hospital. On the other hand, the private sector does not seem to be affected by bureaucracy or administrative bottlenecks. A private doctor could call on their drug representative or supplier and ask for a supply at any time, thereby minimising delays in the supply chain. This may not be possible in the public sector without meetings and other administrative routines. Overall, bureaucracy and other factors could affect drug availability in the public system whereas in private practice, the huge financial commitment of stocking most drugs and their shelf life may influence drug availability at any given time.

\subsubsection{Buildings /equipment}

The participants' judgements differed on whether private practice has more equipment than public hospitals.

We receive quality services in government hospitals not minding the long waiting time there. Government hospital has the best of equipment, and human resources. Everything you can think about is there in the government hospital. (FGD 3, P2)

But after the delivery of this my last baby, I had complication, but because they did not have the facilities to take care of the complication in that government hospital, I was referred to a private hospital. I stayed there for two weeks and was charged N57000 (£139). (FGD 1. P.1)

In the case of equipment, for instance, if an incubator is needed in a government hospital, it may not be functioning at the time is needed but when these private doctors refer you to their hospitals, they will come there and treat you and look after you. (FGD 1, P6)

From the service users' view point, it is not established whether the public has better functioning equipment than private practice. Their perceptions were based on the different experiences in both sectors. While there may be equipment deficiencies in both sectors, tertiary hospitals (public) may have more sophisticated equipment, of the type unlikely to be available in many private practices. But at the lower level public hospitals, availability of equipment may be a challenge. Moreover, some established consultants with private practices may have better equipment than the lower-level public hospitals that are supposed to act as gatekeepers. Nevertheless, there are better buildings and infrastructure in the public hospitals than in most private practices, which typically occupy rented flats in residential buildings. 
Some of the participants were indifferent to physical attribute of quality as long as they achieve results in terms of health gain.

Hospital is not a hotel. We don't expect to have the best of infrastructure, but a result. The hospital should be neat but not in a mess. Your primary concern when you come to the hospital is to recover and get better. Even if the treatment is given in a dustbin as long as the result is achieved, and the person recovers quickly, it does not matter "(FGD 3, P.3).

\subsubsection{Health workforce}

Our participants indicated that there are more competent health workforces in the public hospital than the private. There was a general consensus that public hospital has more qualified and competent workforce than those in the private practice.

You have qualified nurses in government hospitals but not so in private hospitals where government doctors work. As long as you can attend to patients you may be recruited to work there. If you are not a qualified nurse you cannot work in a government hospital, instead, you may work there as a cleaner. (FGD 3, P.4)

Healthcare staffs in the private are not better than those working in government hospitals but because of the constant monitoring and supervision they are able to sit up. Servicom should work hard to check on what healthcare staffs in the government hospital do. The doctors in government hospitals are qualified and they know what they are doing but the challenge is that they are not being checked and monitored very well (FGD1, P).

The typical public hospital has a more competent and specialised workforce than the private sector. However, even though the private setting has a less competent workforce, patient demand and patronage seems to be higher. The desperate patient who needs urgent healthcare may not worry about the competency of the hospital, but in most cases, they rely on friends and family members to decide which private hospital or clinic to visit, or, the reputation of a DP as advertised to them. In private practice, unlike the public system, there is a lack of team work or peer review of colleagues as only the owner manages the clinic or may be assisted by another doctor who often comes in on designated days. 


\subsection{Process element of quality}

The process element of quality involves what is done in the course of delivering care. In other words, it covers the overall process of receiving care. It includes the interactions between service users and healthcare providers. The interactions include waiting time to see the doctor, information given by patients, diagnosis carried out, rapport with patients, communication and so on. We further subcategorized this subtheme into: showing concern for patients (positive) in the two sectors, and other process elements of quality considered to be negative from patients' perspective.

\subsubsection{Showing concern for patients}

Our data show that more positive process elements are in the private rather than the public sector. The majority of the participants were happy with the way they received treatment and attention from private providers. According to them, private healthcare staff showed concern for patients in different ways:

If I came to the hospital and a health worker came out to welcome me to see the situation and how to salvage it, just coming in and showing concern. Unlike that of public hospital $X$ where they did not even come out to show concern, we followed them inside and they said no there is no room. But in the private the doctor came out and helped to bring the patient out of the tricycle. There are two things here; the time may not matter to me but showing concern matters a lot. Their ability to welcome the person shows that at least they can take care of the person (FGD 2, P.6).

If I am given an immediate attention without delays, and how I am welcomed in the hospital will show me that they know the value of life. There are some hospitals that even when they know that someone is dying, and that the person needs urgent attention, they may not give that immediate attention to the person. For me quality of care involves giving more attention to someone whose case deserves more attention in a hospital "(FGD, 4, P.5).

The overall interaction in the process of receiving care was found to be more positive in the private sector than in the public. Though, participant's experience might be subjective, but they represent an independent assessment of how care was received. The result might suggest a higher patronage for the private sector for this aspect of care quality. Nevertheless, this does 
not imply that the process element is always positive in the private sector, though; the frequency might be higher than the public sector.

The respondents had different experiences in how they received treatment in the public sector. Most of them expressed displeasure with the way public providers attended to them. According to them, there were more negative experiences than positive in the public hospitals.

They told me to open my legs wide, if you see what these students did to me, and my husband was barred from entering the consulting room where we were in. We spent many hours there and my husband was wondering if there was an operation going on in there. My husband later got annoyed and followed a staffer into the consulting room and saw how these students hung my two legs wide apart. He shouted at them and asked, is this what you are doing to my wife here? [All laughed]. He then furiously left the consulting room and threatened that I should leave immediately. (FGD 4, P.7).

The experience I had when I was pregnant and visited the public hospital was very bad. The words used by the health workers on patients matter to me. When I was in labour the nurses were making some discourteous statements and I heard them. They were rudely saying that anyone who should get pregnant should be able to deliver the child. They looked at me and I looked young because I have a young face, maybe they thought that I was a very young girl. They should know the words they speak to someone can bring the person back to life or to the other side the world. (FGD 2, P.3).

Providers' attitudes to service users in the public sector scored poorly. The rights of patients are not well monitored or safeguarded. Providers appear to be paternalistic in dealing with patients. While the cost is cheaper in the public sector, patients' experience may be poorer.

\subsection{Outcome element of quality healthcare}

The outcome element of healthcare quality means the effect of care on patients' health status. The expectation of patients, therefore, is to benefit from treatment received. Our finding shows that participants regard the outcome element of care as positive only when their health status improves. According to them, the purpose of visiting the hospital is met when the outcome is positive. This subtheme is further categorised into positive and negative outcome experience in both the public and private sectors. 


\subsubsection{Positive outcome element experience}

The respondents had positive outcome experience in both public and private sectors. It was found that positive outcome experience was not limited to private practice or the public system. The excerpts from the participants are as follows.

I had an experience in a public hospital when I was pregnant and went for antenatal. It was discovered that I had a tumour. A surgeon in a private hospital told me that he would be able to operate on me, but my brother told me that I should not take such risk and should go to a big public hospital for the operation. Nine surgeons did that operation and I was well taken care of after the operation. They did wider incision and brought out the sag to avoid further development of the tumour. If it was in the private, perhaps they would not have been able to carry out the operation very well. That was the reason I registered in the government hospital when I had my last baby. (FGD 1, P.7)

I had a case in a private hospital where I sought treatment for my child. All the drugs I got there did not treat him. But when I went to a government hospital the doctor prescribed some drugs which I bought from the hospital pharmacy. So, when I gave it to the child he recovered immediately. (FGD 4, P.5)

The outcome element of care is regarded as one that improves health status including the level of satisfaction a patient derives from treatment received. Sometimes, outcome may be satisfactory but the process that led to the outcome may be poor. For instance, in time of emergency, during saving a patient life, some of their rights may be violated unintentionally. So, high performance in one element does not determine the level of performance in another. A good healthcare received may not guarantee an improved health status, yet the patient may feel satisfied. More so, the best of care may be given to a patient, yet death may still occur. If our assessment of quality healthcare is based on health status improvement alone without regard to other socioeconomic and environmental factors, and given the complexities of medicine, then our judgement may sometimes be faulty. In some instances, despite a shortterm positive outcome, harm can still become manifest later. While the process seems more obvious to service users, the outcome element of care quality apparently is more technical and beyond the scope and comprehension of service users and their assessments may often be biased. 


\subsubsection{Negative outcome element experience}

It was also found that negative outcome experience was not specific to the public or the private sector. Respondents narrated negative outcome experiences that had occurred in both types of settings.

My first child died in a private hospital owned by a government doctor. How did it happen? My wife delivered a premature baby of about 6-7 months. The child was kept in an incubator but died because the oxygen in the incubator was finished. The question is should the oxygen in an incubator be exhausted when a baby is in there? (FGD3, P.4)

I also had a similar case in a government hospital when I took my daughter there because she had an eye problem. The doctor said that an operation would be carried on her. The operation was later carried out, but it was not successful because after two weeks we noticed that there was still a problem. They had collected the money for the operation and then told us to come back for a second operation and that we should come with the exact amount of money charged for the first operation. We asked them why we should come with the same amount of money when they were the cause of the unsuccessful operation. So, because of that we went to a private hospital where the cataract operation was carried out and it was performed successfully. (FGD 4, P.6)

Something still happened. My sister was operated on at a private hospital and a pair of forceps was left in her stomach for three years without anybody knowing. When she was taken to another hospital for another operation it was discovered that there were forceps in her stomach. The forceps had an abbreviation of the hospital where she had the first operation. We went to the Medical director of the hospital and she said that nobody is above mistake. (FGD 3, P.4)

The complexity and technical limitations of present-day medicine mean that a negative outcome will sometimes occur. Treatments do not always work, and mistakes may be made. Patients could also be the cause of a negative outcome if they fail to keep to drug dosage and time, for instance. Private practice may be more prone to medical error. Its operation often lacks team work and peer review, unlike the public system. However, the system must strive to minimize avoidable medical errors.

\subsubsection{Treatment satisfaction in public and private sector}

There was no agreement among respondents on which of the sectors offers most satisfaction with treatments. While some respondents felt more satisfied with private treatment, others preferred the public sector. 


\subsubsection{Public sector}

The relatively cheaper cost of healthcare in the public sector is the major tracer for satisfaction among respondents. They trust the public sector for genuine drugs, and that builds their confidence for continued patronage.

I prefer government hospital based on my experience. For instance, the public hospital is free for immunization and other health conditions. It is not just that some of the services are free, when they give you medication, your health problem may be solved. (FGD1, P.1)

I prefer government hospital, for instance you can use N1000 to buy drugs there, but in private practice, that may not be possible. (FGD1, P.4).

Why I said so is that public hospital is always affordable. But in private practice I do not think it is always true that you can pay your bills in instalments. (FGD2, P.5).

The public health service is not as business oriented as private facilities. In private practice, financial interest may be one of the key motives. Those who are satisfied with public sector service for affordability may not continue their patronage if their health status is not improved.

\subsubsection{Private sector}

Patients value the respect and quick attention offered in the private sector. There is also a qualitatively different doctor/patient interaction, which is denied them in the public sector.

I prefer private hospital because they give better attention to patients than the government hospitals. When you come to the government hospital, they will tell you that they will call the doctor who is already in his private clinic consulting. And he must finish in his private hospital before coming to the government hospital. You may continue to wait in the government hospital until you die. (FGD2, P.5).

I prefer private hospital despite their charges. If you compare the services they give, you pay the bills with joy. We do not get the kind of services we want in government hospital that is why we go to the private. Even if we pay ten times what we pay in the government in private practice we do not bother because of the services we receive. (FGD 2, P.3). 
Those who need immediate attention, and wish to avoid unpleasant experiences and delays, can opt for private care though at a higher cost. Despite the cost in the private sector, those from higher socio-economic groups are more satisfied with the sector.

\subsection{Chapter summary/conclusion}

The service users' perspectives on quality was mainly centered on the process element as an indicator for good quality care. Furthermore, their responses to the structure element of quality care show that they had a consensus that government hospitals have better physical infrastructure than the private sector. However, the only exception was on the opening and closing hours, which they believed was better in private practice. Their experience in the process of receiving care show that there is more positive experience built around interaction and communication with providers in the private sector than in the public system. In the case of the outcome element, the private sector has more positive outcomes than the public system and overwhelming majority would recommend visiting a government doctor in his private practice. However, given their experiences, some valued private care, and others preferred the public system. Except for cost of service, which generally is higher in the private sector, other characteristic differences between public and private healthcare may reflect shades of opinions based on time and ease of access. 


\section{Motives for dual practice in the public system}

\subsection{Is it all for money?}

This chapter details the ranking of motives for dual practice by dual practice physicians. It also presents the Likert analysis of service users' perceptions of dual practice of government doctors. Results from the different categories of respondents indicating their understanding of the motives for dual practice are discussed. We also present findings on conditions that could influence a doctor to work exclusively for the public sector while the results on motives for non-engagement in dual practice among some public-sector doctors are shown. The challenges faced by DP policy implementation in the Nigerian health sector are highlighted in this chapter. Finally, the findings on the different mechanisms/options to manage DP in Nigeria and the challenges for reform are presented.

\subsubsection{Ranking the motives for DP by government-employed doctors engaged in dual practice}


Table 20: Mean scores for self-reported motives for dual practice by dual practitioners $(n=15)$

\begin{tabular}{|c|c|c|c|c|c|}
\hline Variables & Mean (SD) & Median & Range & IQR & Min-Max \\
\hline Income enhancement & $8.5(1.64)$ & 9 & 6 & 2 & $4-10$ \\
\hline $\begin{array}{l}\text { Low number of hours of } \\
\text { work in pub hosp. }\end{array}$ & $2.3(1.63)$ & 2 & 5 & 2 & $1-6$ \\
\hline $\begin{array}{l}\text { Lucrativeness of the } \\
\text { private sector }\end{array}$ & $6.7(1.75)$ & 7 & 6 & 3 & $3-9$ \\
\hline $\begin{array}{l}\text { Bureaucratic/ } \\
\text { administrative barriers in } \\
\text { public } \\
\text { hospitals }\end{array}$ & $6.1(2.50)$ & 7 & 9 & 3 & $1-10$ \\
\hline $\begin{array}{l}\text { Control of } \\
\text { workplace }\end{array}$ & $5.9(2.31)$ & 6 & 8 & 3 & $1-9$ \\
\hline $\begin{array}{l}\text { Patient shortage in pub } \\
\text { hosp. }\end{array}$ & $2.4(1.68)$ & 2 & 5 & 3 & $1-6$ \\
\hline $\begin{array}{l}\text { Prestige and fringe } \\
\text { benefits in pub hosp. }\end{array}$ & $6.9(2.22)$ & 7 & 8 & 3 & $2-10$ \\
\hline $\begin{array}{l}\text { Professional } \\
\text { development }\end{array}$ & $6.8(2.31)$ & 7 & 9 & 2 & $1-10$ \\
\hline
\end{tabular}

In Table 20, income enhancement seems to be the major motive for dual practice among public sector doctors with a mean score of 8.47 , followed by Prestige and fringe benefits in the public system which has a mean score of 6.93. Professional development came third with a mean score of 6.80. The lucrativeness of the private sector has a mean score of 6.73 while low number of work hour in the public sector has the least mean score of 2.33 .

\subsubsection{Descriptive Likert analysis of service users' perceptions of DP}


Table 21: Service users' perceptions of government doctors who engage in dual practice and why patients move $(N=407)$

\begin{tabular}{|c|c|c|c|c|c|c|c|}
\hline Question & $\begin{array}{c}\text { Strongly } \\
\text { disagree } \\
\text { n(\%) }\end{array}$ & $\begin{array}{c}\text { Disagree } \\
\mathbf{n}(\%)\end{array}$ & $\begin{array}{l}\text { Don't } \\
\text { know } \\
\text { n(\%) }\end{array}$ & $\begin{array}{c}\text { Agree } \\
\mathbf{n}(\%)\end{array}$ & $\begin{array}{c}\text { Strongly } \\
\text { agree } \\
\text { n(\%) }\end{array}$ & $\begin{array}{l}\text { Mean } \\
\text { [SD] }\end{array}$ & $\begin{array}{l}\text { 95\% CI } \\
\text { (lower- } \\
\text { upper) }\end{array}$ \\
\hline $\begin{array}{l}\text { 31a). Doctors employed in } \\
\text { government hospitals engage } \\
\text { in private practice for their } \\
\text { own financial gain }\end{array}$ & $8(2.0)$ & $21(5.2)$ & $4(1.0)$ & $212(52.1)$ & $162(39.8)$ & $4.2(0.9)$ & $4.1-4.3$ \\
\hline $\begin{array}{l}31 \mathrm{~b} \text { ). Doctors employed in } \\
\text { government hospitals engage } \\
\text { in private practice to raise } \\
\text { their poor salary }\end{array}$ & $31(7.6)$ & $54(13.3)$ & $11(2.7)$ & $179(44.0)$ & $132(32.4)$ & $3.8(1.2)$ & $3.8-3.9$ \\
\hline $\begin{array}{l}\text { 31c). Strikes in government } \\
\text { hospitals encourage public } \\
\text { hospital patients to move to } \\
\text { private hospitals where } \\
\text { government doctors also work }\end{array}$ & $2(.5)$ & $6(1.5)$ & $2(.5)$ & $159(39.1)$ & $238(58.5)$ & $4.5(0.6)$ & $4.5-4.6$ \\
\hline $\begin{array}{l}31 \mathrm{~d} \text { ). Delays in paying public } \\
\text { hospital doctors' salaries make } \\
\text { them engage in private } \\
\text { practice }\end{array}$ & $40(9.8)$ & $69(17.0)$ & $28(6.9)$ & $176(43.2)$ & $94(23.1)$ & $3.5(1.3)$ & $3.4-3.7$ \\
\hline $\begin{array}{l}\text { 31e). Lack of equipment in } \\
\text { government hospitals makes } \\
\text { government doctors engage in } \\
\text { private practice }\end{array}$ & $108(26.5)$ & $141(34.6)$ & $20(4.9)$ & $83(20.4)$ & $55(13.5)$ & $2.6(1.4)$ & $2.5-2.7$ \\
\hline $\begin{array}{l}\text { 31f). Government doctors } \\
\text { engage in private practice } \\
\text { because government hospitals } \\
\text { are poorly supervised and } \\
\text { monitored }\end{array}$ & $30(7.4)$ & $75(18.4)$ & $36(8.8)$ & $158(38.8)$ & $108(26.5)$ & $3.6(1.3)$ & $3.5-3.7$ \\
\hline
\end{tabular}

Scores ranged between 1 and 5

Table 21 summarises the findings regarding service users' perceptions of why full-time government doctors engage in DP, and why patients move from public to private hospitals. The Table shows that the vast majority of respondents (91.9\%) agreed or strongly agreed that government doctors engage in DP for financial gain. Regarding the view that poor salaries are a key factor, $76.4 \%$ of respondents agreed or strongly agreed that DPs engage in private practice to raise their salary. Concerning delays in paying doctors' salaries as the cause of DP, $66.3 \%$ agreed with that statement. Respondents considered lack of equipment in public hospitals to be a less plausible reason for government doctors engaging in private practice, 
with only about $34 \%$ agreeing or strongly agreeing that this was a factor. A greater number thought that poor supervision and monitoring in public hospitals might motivate doctors to engage in dual practice, with $65.3 \%$ agreeing or strongly agreeing. Here around $26 \%$ fell in the 'disagree' or 'strongly disagree' categories. On the issue of strike action and how it encourages transfer of public patients to private facilities, almost all respondents $(97.6 \%)$ agreed that this was a factor pushing public hospital patients towards dual practitioners' private facilities.

It would seem that service user's financial motivation is far the most prominent reason for doctors to engage in DP, and that reasons that some doctors themselves give are regarded as less central. Interestingly, the idea that strike action - something that might affect the thinking of service users rather than doctors - significantly boosted DP commanded the strongest agreement of all (Mean 4.5, 97.6\% agreeing). Because of the different distribution of responses between 'strongly agree' and 'agree', 'poor supervision and monitoring' comes out with a higher mean score than 'delayed payment of salaries', even though in total a marginally higher percentage of respondents agree that the latter was important.

\subsubsection{Cluster analysis of study locations}

Table 22 below indicates the extent of the effect that clustering is having, with the DEFF and DEFT columns, providing the design effects (DEFF) and the square root of that number (DEFT). Taking the first row as an example, the DEFF is 0.943 , which says that the variance is 0.943 times what it would have been if a simple random sample had been employed. The large effects (relatively high DEFF) have been highlighted, which for example, changes the picture for Q31D, since a DEFF of 26.981 indicates that the variance is nearly 27 times higher than it would have been if a simple random sample had been used. The questions producing results with a high DEFF are: Q31F, Q32F, Q33C, Q33F, Q34B, and Q34D. It must be accepted that clustering may well have skewed the results in respect of these items and the results therefore need to be interpreted with caution. 
Table 22: Cluster adjustment analysis

\begin{tabular}{|c|c|c|c|c|c|c|}
\hline Variables & Mean & Std.Err. & [95\%_Conf & Interval] & DEFF & DEFT \\
\hline Q31A & 4.226044 & .0414491 & 3.699384 & 4.752705 & 0.943 & 0.971 \\
\hline Q31B & 3.80344 & .1056088 & 2.461552 & 5.145327 & 2.962 & 1.721 \\
\hline Q31C & 4.535627 & .0425599 & 3.994852 & 5.076401 & 1.811 & 1.346 \\
\hline Q31D & 3.528256 & .3301197 & -.6663131 & 7.722824 & 26.981 & 5.194 \\
\hline Q31E & 2.597052 & .1376284 & .8483175 & 4.345786 & 3.865 & 1.966 \\
\hline Q31F & 3.587224 & .2406293 & .5297387 & 6.644709 & 14.842 & 3.853 \\
\hline Q32A & 4.179361 & .0194025 & 3.932829 & 4.425893 & 0.117 & 0.343 \\
\hline Q32B & 2.329238 & .0531847 & 1.653462 & 3.005015 & 0.759 & 0.871 \\
\hline Q32C & 2.528256 & .0696775 & 1.64292 & 3.413591 & 1.035 & 1.017 \\
\hline Q32D & 2.651106 & 0399399 & 2.143621 & 3.15859 & 0.385 & 0.621 \\
\hline Q32E & 4.530713 & .0016058 & 4.510309 & 4.551116 & 0.002 & 0.046 \\
\hline Q32F & 3.985258 & .2409673 & .9234775 & 7.047038 & 16.836 & 4.103 \\
\hline Q32G & 3.938575 & .0130396 & 3.772891 & 4.104259 & 0.058 & 0.242 \\
\hline Q32H & 2.081081 & .0280231 & 1.725014 & 2.437148 & 0.258 & 0.508 \\
\hline Q32I & 4.405405 & .0318746 & 4.0004 & 4.810411 & 0.599 & 0.774 \\
\hline Q32J & 2.12285 & .0162512 & 1.916359 & 2.329342 & 0.086 & 0.294 \\
\hline Q33A & 4.012285 & .037006 & 3.54208 & 4.48249 & 0.588 & 0.767 \\
\hline Q33B & 3.734644 & .0999342 & 2.464859 & 5.004428 & 2.919 & 1.709 \\
\hline Q33C & 3.746929 & .2561802 & .4918502 & 7.002007 & 16.815 & 4.101 \\
\hline Q33D & 3.164619 & .0053487 & 3.096658 & 3.23258 & 0.007 & 0.083 \\
\hline Q33E & 4.162162 & .0265622 & 3.824658 & 4.499667 & 0.277 & 0.526 \\
\hline Q33F & 3.31941 & .3941104 & -1.688238 & 8.327058 & 30.980 & 5.566 \\
\hline Q33G & 4.154791 & .0829827 & 3.100396 & 5.209186 & 2.923 & 1.710 \\
\hline Q34A & 4.452088 & .0202959 & 4.194204 & 4.709973 & 0.287 & 0.536 \\
\hline Q34B & 4.061425 & .1506318 & 2.147467 & 5.975383 & 7.723 & 2.779 \\
\hline Q34C & 3.867322 & .0032841 & 3.825594 & 3.90905 & 0.003 & 0.055 \\
\hline Q34D & 3.734644 & .1539883 & 1.778037 & 5.69125 & 6.604 & 2.570 \\
\hline Q34E & 2.592138 & .0539575 & 1.906543 & 3.277732 & 0.539 & 0.734 \\
\hline Q34F & 2.422604 & .1029164 & 1.114927 & 3.730281 & 2.367 & 1.539 \\
\hline Q34G & 2.167076 & .0217086 & 1.891243 & 2.442909 & 0.151 & 0.389 \\
\hline Q34H & 3.727273 & .1023006 & 2.42742 & 5.027126 & 2.832 & 1.683 \\
\hline Q34I & 3.525799 & .0506855 & 2.881778 & 4.169819 & 0.662 & 0.813 \\
\hline Q34J & 2.189189 & .0247028 & 1.87531 & 2.503068 & 0.178 & 0.422 \\
\hline Q34K & 4.078624 & .0009659 & 4.066351 & 4.090897 & 0.000 & 0.018 \\
\hline Q34L & 4.218673 & .0395414 & 3.716252 & 4.721095 & 0.628 & 0.792 \\
\hline Q34M & 2.34398 & .0091398 & 2.227848 & 2.460113 & 0.018 & 0.133 \\
\hline Q34N & 3.916462 & .1287904 & 2.280025 & 5.552899 & 4.782 & 2.187 \\
\hline Q34O & 2.702703 & .0282887 & 2.34326 & 3.062145 & 0.210 & 0.458 \\
\hline Q34P & 2.405405 & .0270934 & 2.061151 & 2.74966 & 0.194 & 0.441 \\
\hline Q34Q & 3.982801 & .1152678 & 2.518184 & 5.447418 & 3.332 & 1.825 \\
\hline
\end{tabular}




\subsection{Perspectives on motives for dual practice}

This section covers findings on the perspectives of service users of doctors engaged in DP.

\subsection{Service users' views on doctors engaged in dual practice}

Service users viewed financial self-interest and public system failure as the two main influencers of DP in public hospitals.

\subsubsection{DPs make financial gain from private practice}

DP according to the respondents is undertaken for financial gain. The dual practitioner wants to make extra income from private practice. It was gleaned that public-sector doctors are not well paid; therefore, income top up becomes necessary.

I think the reason doctors work in both government and private hospitals is because of money (FGD 1, P.3).

They make more efforts to attend to you in their own hospitals because they know the money they make from that belongs to them. That is my experience in both government and private hospitals owned by government doctors. (FGD 3, P.3)

I do not think that the reason government doctors open private hospitals is because they are not well paid, after all, how can they open private hospital when they do not have the money? I think the reason is for their own financial gain, there is no one who does not open a business for profit. So, in the case of public doctors they want to make more money, not that they are not well paid. (FGD 4, P.3).

It has been noted that financial self-interest is the overall reason for dual practice. It might be put forward as a justification that poor salary is the major motive for dual practice. This is also exacerbated by the austerity measures in most developing countries, including Nigeria. In high income countries where medical professionals earn good income, relatively, it might be questionable to attribute dual practice for income top up. The Purchasing Power Parity (PPP) across countries (developed and developing) where DP is practiced seems to be in disequilibrium. A few pounds in the UK for instance, could pay a junior doctor's salary in Nigeria, yet DP is practiced in the UK for the same economic reason of beefing up public- 
sector salaries. However, there might be another driving force for DP that may worth investigating.

\subsubsection{Public system failure}

Apart from financial self-interest, organisational shortcomings of the public system may be a reason for dual practice. They viewed the late payment and poor salary of public sector doctors as important tracers for DP, often orchestrated by the failure of the public system. It has also been noted that strikes in government hospitals create the opportunity for dual practice.

I think it is because government does not pay them well, that is why they engage in dual practice. If you do not pay those who work for you, the person has to look for something else to do to support himself. The government at times do not pay their workers well and as at when due. That is what I think drives them into private practice. (FGD, 4, P.3)

It is also due to strikes in government hospitals that make doctors to establish private practice. What these government doctors do is that from time to time they ask government to increase their salaries and when these demands are not met, they go on strike, and when they are on strike, it further helps to push patients to their private practices (All agreed). (FGD, 4. P.1).

There seems to be a tension between the professionals and the public system. This tension is evident in a number of strikes often embarked upon by medical professionals in Nigeria over salary increase. The medical profession accuses the public system for the poor health system in the country and most often exonerates itself from any blame. However, salary increases in a system already under austerity like Nigeria could have a spiral effect as those in other professions might demand salary increases as well. The more dual practice is multiplied, the more the healthcare delivery in the public system suffers.

\subsection{Doctors' views on dual practice by public sector doctors}

Both dual and non-dual physicians' respondents mentioned different areas they think are reasons for engaging in dual practice. The major areas discussed were the bureaucracy in the public hospitals, income enhancement and poor working conditions in the public system, skill 
acquisition and skill utilisation. Each of these is discussed in this section.

\subsubsection{Escaping bureaucracy in the public system}

Respondents mentioned two important areas of bureaucracy in government hospitals. Administrative delays in public hospitals were said to be due to administrative hiccups created by hospital bureaucracy. To them, patient delays are not the doctor's fault.

And if there is anything you need for your work, you have to pass through chains of bureaucracy before your demand gets to where it will be assessed before it gets back to you. Then, you might not be able to access the item you requested for your work for a period of time. There are occasions you may be denied that thing because the person that needs to access it and send to you may not know the use or the importance and you end up not having it; the person that bears the brunt is always the patient. The item is treated like a bureaucratic 'thing' and not like something that has to do with patient management and life. (Interview with a nonDP Snr. Registrar)

Invariably, all this affects the smooth running of our healthcare services. You may well get frustrated. Let me give you an example. If I book a patient for a surgery and make a request for an item I need for the operation, you may be surprised that you will go there only to find out that those materials have not yet been supplied. Then you must go shouting 'who is supposed to do this supply?' probably the pharmacist, and he will give you a flimsy excuse and you must shout at him because you are the one that wants this case done. At the end of the day, it may be supplied, and you want the patient to be moved to the theatre, and you keep looking around asking who is supposed to move this patient to the theatre. Can you imagine the surgeon doing all this? When you now get to the theatre, an anaesthetist who's supposed to receive this patient is not there and you cannot do the surgery without anaesthesia. You have to run around shouting and looking for the nurses on duty. You can now see the stress to do just one case. But, in a private setting, you are in charge, it's just a case of give me this, you just write, and it is efficient, and the patient is there with fewer hiccups. When you spend all this energy just to start a procedure, what's left for you to carry it out? (Interview with a DP Snr. Registrar)

The respondents suggested that hospital rules and regulations affected doctors' performance. They argued that the formal hospital guidelines for doctors and the involvement of nondoctors in the treatment process, who probably do not understand professional ethics, impact adversely on doctors' performance in public facilities. 
Again, they may tell you that the drug you requested is out of stock; instead of looking for the drug to send to the doctor, so that they can work with it, someone goes outside to buy it. The drug may be there in the hospital, but it is just that they may be lazy to look for it, or you find someone else who will tell you that there is no linen to carry out your surgery or that the linen is washed, but not sterilised. Or, you may find another person telling you that the instrument you need is not there - there are a lot of bottlenecks even when you have come to do your work; you come in the morning and you're ready to do your work, but people are frustrating you here and there. (Interview with a DP Consultant)

From the doctors' accounts the application of civil service rules meant to prevent abuse of the public system compromise their clinical work.

\subsubsection{Income enhancement}

Generally, all the respondents agreed that income enhancement was the main reason for dual practice. They mentioned that government work does not pay well and that they must augment their pay.

Well, you see, the major employer of labour in Nigeria is the government. And the major employer of labour does not pay well. Government at best employs only about $2 \%$ of the specialists that are turned out yearly. Who will employ the others? Maybe the state government, the state government is not even employing, their case is even worse. Even when they employ, they do not pay. For people to remain in the government hospitals and be able to render the services needed to train medical students and resident doctors, somehow, they use dual practice to augment their take home pay and that encourages them to work. (Interview with a DP Consultant)

Like I told you before, the economic incentive in government hospitals is very poor. And, because of the poor incentives, people have to make up for the poor salary in the public sector to be able to stay afloat. The pay here is very low and, because it is very low and, if you have a family - especially an extended family system for that matter, you must pay school fees for nephews, nieces and cousins and aunts. You must cater for your extended family and your own children. And what we are paid here in the state simply cannot make that happen. Most of the people who do private practice do that to supplement to keep afloat. (Interview with a DP Snr. Registrar)

Income may help dual physicians to make up for poor public-sector salaries. The implication, however, is that, if more money is made in the private sector, more attention might be shifted to private work. The fear is that truancy and absenteeism in government may increase. 


\subsubsection{Poor working conditions in the public sector}

One of the claimed motives for DP in the literature review is poor working conditions in government hospitals. The respondents argued that working conditions in government hospitals are bad and that the conditions serve as push-factors vis-a-vis private practice.

It is everybody's right to be able to work to the utmost ability that he has. And, if the employing institution does not create that environment, it is a sign of high responsibility for the employee to try to use every other opportunity to give the kind of training he has been trained to give. So, if you try to limit that in any way, you are both limiting the person as an individual and undermining the whole environment - both the service receivers and the service providers. In that respect, it is clear that there is actually no reason why there should be no dual practice as a way for government to accommodate its own inadequacies. (Interview with a DP GP)

We have a lot of challenges in our healthcare. As you know, there are a lot of problems in our healthcare system. You find, in most cases, that the politicians and policy makers go outside the country in search of healthcare even though we have hospitals back here. If you look at the package for these health workers, it is disheartening when you compare it to the efforts to save lives and the facilities available to offer efficient healthcare. Most of them, if they are available, are in the state of disrepair. And some of them that should be affordable are not available in some of these tertiary hospitals. (Interview with a DP Snr. Registrar)

Modern diagnostic equipment is lacking in government hospitals and that affects service delivery in the public system. Historically, the conditions of work in government hospitals have not met the expectations of doctors working in the public system. From the standpoints of doctors, if work conditions are not improved, then extra private work should be justified.

\subsubsection{Skills utilisation}

Skills utilisation means that skills developed have to be used to benefit the doctor and patients. Respondents were of the view that a doctor should work in a setting that tests their skills.

Sometimes you are in a place where your career... like I said, I have a subspecialty in respiratory medicine, but since I came back it is not being practised. We do not have the equipment; nobody is even bothered since I came back from the training, because I did the training in South Africa. So, is it a big 
deal if I can get my own equipment, source it by myself and get a private place and get fulfilment to practise what I was trained in but which the public sector has not given me the opportunity to do? So, I'd rather set up a private outfit rather than die practising nothing, yet I spent my whole time and resources, left my family, travelled abroad and acquired knowledge, came back and cannot put it into practice. I don't have the money, but if I did I'd set up my practice, give people what they need and get fulfilment. (Interview with a non-DP Consultant)

According to these respondents' government hospitals under-utilise doctors' skills due to poor or absent equipment. The private sector gives them the leeway to utilise the acquired skill.

\subsubsection{Skills acquisition}

Some respondents contended that government hospitals lack the volume and mix of cases necessary for training purposes and said that private facilities were better in that regard.

Two, is that the hospital may not be at its maximum in terms of patient management and skill acquisition because the doctors are not seeing as many cases that they are supposed to see to enable them to enhance their practice. (Interview with a non-DP Consultant)

I remember as a house officer, I had the opportunity of working in a private hospital where I had to watch how a caesarean section [CS] is done first-hand. It also gave me the opportunity to see first-hand how evacuation is done. As you do it, you are improving on your skills. When I then went for the National Youth Service, it became an advantage for me because one of the hospitals in the state where I served knew that I was much more experienced than the medical officer who was there before me due to the skills I acquired from private practice. Like I told some doctors here, when you go there [private practice], do not start looking for money, but sharpen yourself up because you don't know where you will end up. So, you go there and learn these things and you save lives with them. (Interview with a DP Snr. Registrar)

Private practice, no doubt, may help doctors to acquire skills as it has the potential to expose doctors to cases that may not be available in the public sector. By and large, cases that may not be common in the public sector could be seen in the private sector. Doctors who straddle both sectors can gain additional experience and skills. The main question, therefore, is to what extent the skills gained in the private sector can help the public sector, which is the main employer. 
Other informants were sceptical of these claims.

Yes, to gain more skills and experience, but, sometimes, it is to satisfy their senior colleagues, because some of those hospitals are owned by consultants and professors. Some use it to do 'eye service' [lip service], doing a kind of advocacy to please them; some of the consultants may not pay the resident doctors who go to their hospitals to cover them when they are not around. When you go there to meet them, sometimes they can teach you the secret of the job, something which they might not want to teach you in the public hospital. So, apart from the financial reason, some of them go there to gain more skills and to satisfy their consultants, who are equally their examiners, because these are the people that will come to examine you. Some of the doctors I know, apart from the monetary aspect, go there to satisfy the consultants, because, if you are doing residence in Obs and Gynae, for instance, and want to engage in private practice, it has to be in your consultant's hospital. You must do that and when you do that you are trying to satisfy them; sometimes, they might not even pay you or they might pay peanuts. (Interview with a non-DP Snr. Registrar)

But I have also found out that, sometimes, some of the people who own private practices still utilise the resident doctors. Like when I used to work in the newborn hospital, my registrar would come late to work, and he would tell me that he was covering someone. He was my senior, so I could not have issued a query or scolded him. I would just say, 'guy, when you are on duty with me, don't do that.' But you cannot fight as you should, because he was working for a more senior person [a consultant] and that affects his work. (Interview with a non-DP Consultant)

\subsection{Policy makers'/stakeholders' views on doctors engaged in dual practice}

The respondents attributed motives for dual practice to many factors. The main areas they perceived as potential reasons for dual practice among public sector doctors were: bureaucracy in government hospitals, economic pressures, poor conditions in public hospitals, and community demand for private clinics. Each of these subthemes is discussed below.

\subsubsection{Bureaucracy in government hospitals}

Bureaucracy was perceived as one of the main causes of dual practice among government doctors. They hold the view that bureaucracy forces doctors to the private sector where there is less interference. Respondents suggested that bureaucracy, though, a means of getting 
government's work done, limits doctors' performance. Some of the extracts from the interviews are below.

...then on the other hand too, because of bureaucracy in the public sector, certain clients also find it more convenient to just walk into the private clinic, see the doctor and they do not have to go get the card in the medical record, pass through the pharmacy when given a prescription, line up for drugs. It also encourages these doctors in dual practice to focus more on their own private practice. (Senior officiall State Health Board)

There is a problem of complete loyalty of doctors employed by the government especially when you add the challenge of bureaucracy and other challenges you have in government facilities. For instance, a surgeon may want to perform a procedure in a government hospital and there could be delays. These delays may be caused by non-availability of the person in-charge of the item you need, say oxygen, the person might be in a meeting, and the next day they say that the person who should complete this form is not yet ready, and you place the surgeon on top of the patient he wants to handle. He has the technical knowledge and when there is this bureaucracy and delays they create a situation where there is that tendency to move to a setting where there will be less delays and bureaucracy (Executive Officer1, Nigeria Medical Association).

The opinions of the respondents reflect the idea that public hospitals suffer delays caused by government administrative procedures. While bureaucracy remains an integral part of government institutions, respondents suggested it has drawbacks in terms of poor response to clinical decision making. Bureaucracy in government hospitals affects both patients and doctors, and there was a perception that private settings can minimise its effects so as to avoid interfering with service delivery where time is critical for saving human life.

\subsubsection{Economic pressure}

Economic pressure was viewed as one of the reasons for dual practice among government doctors. Respondents held the opinion that doctors' take-home pay is not "enough to take them home", thus encouraging extra income generating activity. It was also said by some that there is a disparity in doctors' wages across public hospitals, even for those receiving the same level of training. The pay disparity depends on doctor's primary place of work - local, state or federal - which has different payment structures. 
The main problem as far as I know is that some people say that they want to find a place where they can keep their services going, but the aftermath of everything is extra finances and extra money. So, if you see somebody that pays you well, why are you going to worry yourself to do extra job in the evening when you can stay with your family and then do some other social thing. (Senior Official, Ministry of Health)

What do we see today in our states? Like I said we have different levels, and they all pay different wages. Imagine a doctor at the local government level, for instance, earning the sum of N50,000 [£105] monthly, then a doctor at the state level earning N100, 000[£210] and at the federal level going home with N150, 000[£316] monthly. You see the disparity; it could be that they have the same professional experience but earning different packages. Many of them may look elsewhere to make up. (Senior Administrator, Medical Council)

Economic pressure has the potential to affect doctors' work in the public sector. Low wages coupled with inflation puts pressure on living standards and pushes government doctors towards dual practice. However, it is not guaranteed that doctors would work exclusively in the public system even with higher pay. While a pay rise was cited by many respondents as the obvious means of keeping doctors in the public sector, others mentioned non-financial incentives such as an improvement in staff working conditions, or the provision of soft loans that could assuage financial pressures.

However, higher incomes may have an attractiveness in their own right that is not just about the adequacy of low wages, and the fact that many doctors in high-income countries seek to supplement generous public-sector salaries through additional private work suggests that achieving a basic income threshold may not halt dual practice.

\subsubsection{Poor conditions in public hospital}

Government hospitals do not have all the necessary equipment for efficient service delivery. In some instances, private hospitals are better equipped than government hospitals. Basic medical equipment is lacking in many government hospitals.

Several respondents suggested that work conditions in public hospitals affect doctors' performance. They opined that this might be why some doctors prefer to operate private clinics or hospitals where they can work with superior facilities. 
...but we do know that in our own environment because things do not go the way they ought to. You find out that the public facility that is supposed to be of a standard does not have some of the equipment or drugs needed to take care of patients. In most cases, some private facilities are better equipped than our public health facilities. For instance, you see that a public health facility does not have a CT scanner, but a private facility owned by a practitioner working in the public facility would have it. (Senior Administrator, Medical Council)

The third is the work setting. Can they [the doctors] do their jobs in the standard way? Is there electricity, support staff, facilities and all the things they need? So, if you put a good facility whether private or public, the doctor will naturally go there and do things. (Executive Officer1, Nigeria Medical Association)

However, one senior administrative officer expressed a contrary view. According to him, some of the claims by dual practitioners about public hospital are a ruse to justify their private practice, when the real motive is financial.

...Do you think that an individual is richer than government? Definitely, not true. Let me give you one example, in one of our district hospitals, one of the doctors there prefers doing appendectomies in his own private clinic instead of the government hospital. We accosted him one day and asked him why. He said that there was nothing in the government hospital. We said ok, we went around and opened the store of the government hospital, there was an electron autoclave, and an anaesthetic machine, diathermy machine, and blood bank, all packed in the store. We said no problem; let us go to your clinic. When we got to his clinic, he was using a kerosene stove to sterilise instruments he uses for surgery. Therefore, what will you say about that? You see that was a self-preservative mechanism, he wanted to justify why he was doing that; of course, we sacked him from his job in the government hospital. We asked him since you have better equipment in your private clinic than the government, go and use it, and we went ahead and brought out that equipment from the hospital store and set it up. (Senior Administrator, $\mathrm{MOH})$

The working conditions in the public hospitals may suggest that they are not performing optimally. From the perspectives of many policy makers and stakeholders, public hospitals cannot deliver efficient health care services as they are poorly equipped. They were of the view that the private sector offers better working conditions, and that doctors naturally would like to work where they have better facilities. However, a contrary view came from one of the senior administrators in the Ministry of Health who challenged the claim that government hospitals lack facilities. He believed government-employed doctors use such claims to justify 
their private work and suggested that private clinics, mostly run by government doctors are less well equipped than government hospitals.

\subsubsection{Community demand for private clinics}

Some respondents said that doctors may receive requests from people in local neighbourhoods to open a clinic. According to them, potential private patients expect to be seen in a clinic rather than just going to the doctor's house for a medical check-up. They suggest that it is a concern to help sick people in settings convenient to them that draws some doctors to dual practice.

... You know, when you are a professional it affords you a whole lot of opportunities. When you are a professional, you are developed, if you distinguished yourself, people look for you. When you finish your work and you want to rest, people still come and look for you in the house, and you know this is an issue of life and death, and the issue of helping to save life. Of course, you are doing well by saving life and small money also comes in. It is a natural thing. Sometimes, when the pressure gets high, the doctor may want to get a small clinic, He then opens a clinic so that some evenings or a given time, he goes there, and people come in and he attends to them. It tells the story. It also comes with the issue of abuse. For instance, if I can actually see patients and make a N100,000 naira [£211] in three days, that means assuming I do it for ten days that is

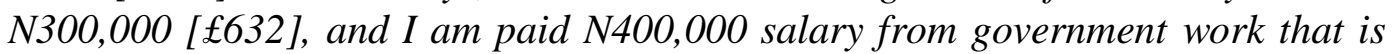
N700,000[£1474]. Then when the limit is not defined, abuse sets in. Therefore, it is a thing of mind. (Senior Official 1, State Health Board)

In a healthcare system with a poorly developed health insurance market like Nigeria, most people obtain health care through out-of-pocket spending. The behaviour of doctors is therefore crucial in every neighbourhood. People seek them out for help even at inconvenient times, especially in the event of emergencies. For some doctors, opening a clinic seems to be a way of escaping from neighbours and family friends coming to consult them in their houses. Although there are many private practices in towns and cities, the WHO doctor-patient ratio of 1:1000 is still unrealizable. Ordinary people may experience times when it is hard to get medical attention. Therefore, demand from the local community could be a factor pushing doctors towards establishing private clinics. This drive however, needs to be balanced to safeguard the public system. 


\subsection{What conditions could make a doctor work exclusively in the public system?}

This section looks at the conditions under which DP physicians might return to full-time public work and give up dual practice. Respondents mentioned several possible changes that might lead them to give up DP, including: reducing bureaucracy in the public hospitals, increasing doctors' remuneration, improving the conditions of work and providing the opportunities for medical updates and conferences.

\subsubsection{Reducing bureaucracy in public hospitals}

Bureaucracy was seen as a problem that was increasingly affecting doctors' work in the public sector, with highly negative consequences for efficiency and morale.

Of course, that is the main thing. It is the bureaucracy, because things are not provided in a timely manner. I'll give you an example, let's say, you are arranging for a procedure and you need 100 naira to do that, and then it is a public hospital thing and the management, with its bureaucracy, are able to provide N98; they have got everything, but without the remaining N2 the procedure will not take place and then, because of the bureaucratic nature, it may take three months for you to get that additional N2 or 2\%. (Interview with a nonDP Consultant)

Sure, we know that administrative bottlenecks can affect practice negatively. You want to get something, it has to be that this person must do this, and that person must be informed, and precious time will have been lost. But, in a private practice, you say this is what should be done; one individual can even do two functions and get the thing ready in record time, and you are happy, the patient is happy. One other thing is that patients hate delay before treatment. They hate being kept in suspense for endless number of hours before the final treatment starts, nobody will like it. It is a good point for dual practice. (Interview with a DP Consultant)

Bureaucracy in government hospitals impinged on clinical work to affect both doctors and patients; several respondents indicated that public work would be much more attractive if it could be reduced. 


\subsubsection{Opportunities for conferences and medical updates}

Some of the respondents pointed out the need for opportunities from government to enable them to attend local or international conferences and seminars. They discussed the importance of attending seminars and conferences and how this would help them keep abreast of cuttingedge medical knowledge.

Providing opportunities for conferences, some people can go for updates. If a person does not go for updates, then there is a problem. I travel every two to three years to work in a world-class cardiac centre and this is my money and it is not sustainable. Not everybody can do that. They should provide incentives so that doctors can go for conferences and seminars and in doing so they can reap the benefits of what they are doing and will be happy working. Any workforce that is happy gives you good results. (Interview with a DP Consultant)

There are other incentives. There are other opportunities, like, once in a while, if you are being sponsored to go for training either abroad or even internally. If these things are made available, you may not think of doing private practice. It will be very difficult. After all, having private practice is energy sapping. (Interview with a DP Registrar)

We saw above how career development is important for many respondents, but even for those who eschew DP may have limited opportunities to attend conferences and seminars; improving this situation would further motivate career-minded younger doctors to concentrate on public-sector work.

\subsubsection{Availability of facilities}

Several respondents opined that the availability of better medical equipment and related facilities in public hospitals could help keep doctors working there.

...the equipment we need to get good results is lacking. What do I mean? For example, a woman in labour needs to be monitored, the monitoring devices are either not functioning or not available and some personnel are not well-trained to man all that. So, most of the time you are working with nothing and you are expected to produce results, to deliver (Interview with a DP Consultant).

If every facility I need for training and working is provided, the patient loads available, and my remuneration is worth my inputs, why stress myself in private 
practice. If all I need to work with is provided in one setting, why would I stretch myself beyond...? (IDI with a DP Resident)

Against the present background of poor facilities in public hospitals many respondents give this as a reason for working part-time in private hospitals and clinics; conversely, some say that better resourcing of equipment in public hospitals would remove the 'pull' of supposedly better equipped private facilities.

\subsubsection{An increase in remuneration}

The consensus was that doctors desire pay rises. They hold the view that, with a good salary package from the government, there will be no need for any additional private work.

Many people will decide to do only public work if government increases salary. Many will be more committed and decide to stay in the public sector, only probably going into private practice when they are about to retire or something like that. If the package is sufficiently good enough that you do not need an extra stress on yourself, you get more committed to research and teaching and give all that you must give. You can stay in the hospital till late in the evening to be with students and do other things. You know it will be more fulfilling and, at the end of the day, you don't really need an extra private practice. If that is done, more people may spend more time in the public sector than the private sector. (Interview with a DP Consultant)

If the pay is good enough and you are getting what you think you should get, money is never enough, really, but, if you are meeting your primary responsibilities to your wife, children and your dependants, and to yourself, although not even those engaged in DP may meet those responsibilities. If you think you are comfortable with what you are doing, I do not think anybody would want to stress themselves further. (Interview with a DP Consultant)

Some respondents disagreed with the notion that increased pay would reduce DP in Nigeria. They suggested that the idea the government would fund such increases was unrealistic and argued that since money was not the only motivation for DP some doctors would still take that path for other reasons.

Now, because of corruption, I do not think that there is any condition that could make me work in the public sector alone. I do not think that those conditions will ever be met. Now we have been talking about our salaries and how to tell government they won't meet these conditions. So, there is no point; they cannot meet these conditions. (IDI with a DP Consultant) 
It might if the only reason for the dual practice is economic. Like I told you, there are many other reasons one may want to engage in DP. Job satisfaction is also part of it, for instance, if I run a private practice in my village where I visit on weekends and I know that people are there and there is no hospital there, a salary increase will not make me stop because I see it as a community service. (IDI with a DP Consultant)

Increased salaries may not stop dual practice in Nigeria. Human want is insatiable, and the Nigerian economy is not getting better. Today's pay rise may not take care of tomorrow's demands.

\subsubsection{Improved conditions of work in the public sector}

Doctors were generally critical of working conditions in the public sector. As with equipment, this was a factor that was seen to reduce commitment to public sector work, and again it was argued by some that improving this situation would pull doctors back to the public hospitals.

Finally, there are the working conditions, just as I am talking to you now, some of us do not have offices in this place. It is mindboggling, some of us do not have any place to stay, you come to work, and you will be roaming about like a cow that has escaped its Fulani master. It is very painful. The welfare of the doctors is just not there, and then another thing is the patient. The facilities for the patients are not there. You come to the hospital and you will not see water and light is hopeless. What do you expect? But, if they can put all these together, then what I am doing in the private setting? (Interview with a DP Consultant)

Yet, as with equipment and salaries, there were respondents who doubted that government would take action of improving working conditions.

There is no way that one day these conditions will be met. We do not have offices here. If I want to write up my research, I go to the private sector. You can now see that nothing can make me stay here [public sector] and work alone [without private practice]. So, carrying out my research is not possible and doing my work and doing it well is not possible. So, the only condition that can make me stay here is to pay me what other doctors in federal hospitals are paid and, of course, then I will sleep here. Why then would I need to be running up and down, provided the agreements are reached? (Interview with a DP Consultant) 
Again, the issue of government resources and investment in hospitals came up. Given the multiple competing demands for improved hospital infrastructure, better availability of drugs, higher salaries and the rest, respondents remained sceptical or at best uncertain about the likelihood that this aspect of the work would improve.

\subsection{Reasons for an exclusive public work by non-dual practice physicians}

In this section we summarise the reasons that non-DP doctors gave for working exclusively in the public sector. The main considerations mentioned were avoiding stress associated with private practice, involvement in research, being content with a public-sector salary, family issues and career development.

\subsubsection{Avoiding the stress in private practice}

Some respondents pointed to the stress they believed private practice brought, and said they preferred to avoid the hassles involved in managing private clinics.

This is because once you start a dual practice, you find out that it takes most of your time, when you work there, you go there, and the stress is much. (IDI with a non-DP Registrar)

Number one is time, if you do not have the time to engage in dual practice, then don't do it; it is strenuous. It is not easy, you need time to rest. I cannot be shuttling between public and private practice. You may be in the public hospital and receive a call that your attention is needed on the other side and you want to rush to the place to find out what is going on there. You will not find time for yourself, family and others and you will not be able to rest and, before you know it, you are worn out, even though, you are making the money. (IDI with a non-DP GP)

Arguably, medical work in both sectors brings stress, but for some doctors at least this can be minimised by avoiding the business-side of medical work, which they do by remaining in wholly public employment.

\subsubsection{Contentment with public salary}

Several non-DP respondents agreed they are content with their government salary and do not need to augment it with private practice. They believed, if the salary is well-managed, it can 
take care of their basic needs.

As I said before, the opportunity cost of the extra hours you put into private practice must be paid for somehow. For me, by the Grace of God, I have contentment with what I receive from the government. Life is not all about money and money does not give life. It just gives you the things to walk around in and makes you more comfortable. But, by and large, I think that I am okay. (Interview with a non-DP Consultant)

Some feel that this money [government salary] is enough if it's well-managed. Like me, I do not really have any private practice except once in a while when I assist in a surgery. Apart from that I don't. It is always a personal thing. (Interview with a non-DP Snr. Registrar)

Some respondents had briefly tried private practice to get extra money but had concluded that coping as they were was a better option than making the lifestyle changes and accepting the pressures that extra part-time work would entail.

Now, the financial remuneration in the public sector is enough for me. I do not want to complicate things by engaging in dual practice. I tried dual practice when I started my residency, I was covering about two hospitals, going up and down, but, eventually, by the third month, I was so tired, so devastated that if I came to the ward, I would be sleeping and lacked concentration while seeing patients. (Interview with a non-DP Snr. Registrar)

It also limits your rest and study periods. If you start it too early [in your medical career] it will affect your professional career. Remember, we are human beings, we need our rest time. It can even eat into your time for research and study. It is a two-way thing; if you overdo the dual practice it can affect you greatly. (Interview with a non-DP Consultant)

The reasons for accepting life on a public-sector salary could thus be manifold, but generally involved the calculation that overall the cost of DP to personal life, well-being and career outweighed the financial benefits.

\subsubsection{Family issues}

Family commitments were mentioned by most female respondents as the main reason for not taking up private work. For them the obligations of the maternal family role - child care, the school-run and participation in their children's social activities - meant that the time was not available for DP. 
It is a decision. If you do a study of doctors engaged in dual practice, you will find out that men do dual practice more than women. For the women, especially for those who are married and looking after their children, the tendency is that they come early and finish before $2 \mathrm{pm}$, so that they can go back to take their children home from school and make sure there is food at home. So, family pressure is a challenge to married women to engage in the dual practice. It is what keeps them in check. For you to say that you will not engage in the dual practice is a decision you take. The women will prefer to come to the public sector and work, and men will prefer to work in the public sector first and then try to engage in private practice. It takes the mentality of a decent human being to choose where they will work and give their best to the place. (Interview with a non-DP Consultant)

Family pressure will make it difficult for you to start looking for a place to do private work after you finish from the public sector. (Interview with a non-DP Consultant)

I have some toddlers. I am a woman and I have a family. My home is there, and I have some little kids around me. That is my own dual practice for now [she laughs]. I have three kids and I have to take care of them. (Interview with a nonDP Consultant)

Nigerian cultural norms about the role of women in family life result in a pattern where far more men than women doctors engage in DP.

\subsubsection{Career development}

Career development in medicine may involve study, additional professional examinations, academic publications, engagement in activities such as seminars and conferences, and involvement in professional associations. Several respondents considered private practice to be a distraction from their professional development.

I was once engaged in dual practice before I stopped. For your development, you need time. My reason is for my development. I am a neurologist; we have a very busy practice and if I have to combine it with the dual practice, I may find it difficult to develop in my career. I remember that with dual practice travelling can become difficult for you. I have done it and the extra income can be very useful, but that is not all the answers. I feel that if I can manage what I have [government salary], it should be able to meet most of my basic needs, but I cannot develop the way I want to develop with private practice. If, in the next three to four years, I have reached the level I desire in my professional career, I 
may go back to dual practice. I feel it is too early to go into that because it will affect my development. I have put DP on hold. (Interview with a non-DP Consultant)

I decided that, because I want to concentrate on my residency training, maybe when I become a consultant, I can go into private practice, but now that I am a senior registrar I just need to do my project [fellowship project]. I find the reason why many doctors spend a lot of time in residency training is when you are trying to combine them [private practice plus public work plus residency training]; at the end of the day you have a divided attention. The little time you are supposed to use to study at home or elsewhere, you spend it going to work in the private sector. (IDI with a non-DP Snr. Registrar)

Dual practice could pose a challenge to career development, especially for the young doctors who are still in training with ambitions to become specialists and consultants; many delay DP until later in their careers.

\subsection{Challenges in combating dual practice in Nigeria}

Respondents mentioned various challenges that make it difficult to introduce policies to control dual practice. These include: corruption in the healthcare system, funding/logistics difficulties, a lack of will to institute real change by the authorities, poor medical pay, problems of controlling professionals in autonomous practice, and the weak sanctions available for those who are caught breaking the rules.

\subsubsection{Corruption}

Respondents were of the view that corruption is widespread in the healthcare system, and that of the many who flout the rules few are punished. In some instances, doctors who break the rules seek help from influential sponsors to get disciplinary action stopped.

The truth is that poor monitoring by the supervising agency is a big constraint on the implementation of dual practice policy. Poor adherence by doctors themselves is a problem but enforcing discipline is the responsibility of the hospital management board to discipline erring medical practitioners. Do they do that? They do not. Then they complain of political interference. If you write a query to a medical officer because he was not on duty when he supposed to be there, then those of us on this other side of politics would ring you (the person issuing the query) and say, "Hey! Stop! He is connected". So, the connectedness of doctors 
particularly when you have female medical practitioners who are married to politicians means the capacity of the supervisors to sanction is weakened (Chair, House Committee).

The sanctions are very weak, you give him a query and before he finishes answering the query, somebody who knows somebody is already calling you that he is a relation of this person [political figure]. (Senior Administrator, $\mathrm{MOH}$ )

I will say that we have had few reports. Now because of our environment, quite challenging - impunity takes place. People vested with authority to perform some roles are not performing those roles. You find out that there are laxities in these institutions. (Senior Administrator, Medical Council)

Political connections seem to play a major role in weakening sanctions for doctors who use government time to do private practice. Political connectedness is a term used to show how someone who is connected to an influential person in government gains immunity from punishment for their action. This widespread corruption weakens those who enforce the rules and allows rule-breakers to behave badly with little fear of sanction. Respondents suggested that in a situation where some are punished and others with 'connections' go scot free, the "system" has failed to give the DP regulation the needed support.

\subsubsection{Funding and logistical challenges}

Funding and logistics were mentioned as one of the major impediments to the curtailment of dual practice.

The challenges are enormous. First, of all, is the challenge of funding for visits. About one year ago, the governor donated 11 vehicles. We gave seven to the districts and four remain within the board to enhance visits. These CEO now have vehicles to go and see what is happening. Funding remains part of the problem and mobility also to ensure that the people who are supposed to be supervising are not themselves culprits in the dual practice. The supervisory team may not be committed to fight this cankerworm and of course, the basic issues of, distance and road accessibility to certain facilities, especially during the rainy season. These are the challenges. (Chair, House Committee)

Yes, there are challenges, sometimes, they do not have adequate materials in terms of logistics/funding and for you to check some of these practices you have to go out, move to visit places to look at what is happening to see what is not being done right and see how you can correct them. (Director MoH) 
Respondents explained how logistical problems and limited resources constitute a major impediment to the supervision of government doctors in the public facilities. Funds are often absent to purchase the vehicles and or support the visits away from base that would be necessary to monitor what doctors are doing on the ground. However, it might be argued that the non-availability of funding reflects the low priority accorded to facility supervision. The implication of poor funding is that most facilities will not be visited, so that the extent of truancy and absences is difficult to ascertain. Enforcing the rules limiting dual practice costs money and there was a perception among stakeholders that this has yet to be recognised by the authorities.

\subsubsection{Lack of will to enforce rules}

Most respondents believed that the widespread non-enforcement of the rules limiting dual practice can be traced back to a lack of determination by the authorities to tackle the problem. According to the respondents, an adequate regulatory framework exists on paper, but is routinely ignored.

I believe that if such rules are adequately applied, this dual practice may not be a serious problem. Only that sometimes people are so soft about the implementation of rules and regulations, otherwise the civil service rules are quite clear on what people should do. It says the time you should come to work and says the time you should leave at the end of the day. (Director $\mathrm{MOH}$ )

He [a public-sector doctor] is permitted to operate a clinic with a colleague so that in the evening you go out to supplement income from the public hospital. The problem is not the issue of regulatory laws and regulation; it is the implementation of these regulatory laws and compliance. (Executive Officer 1, Nigeria Medical Association)

It was suggested that some organisational superiors who were responsible for policing dual practice regulations may themselves be breaking the rules.

... not just having the so-called regulation for the sake of having a regulation, even the people that supposed to police this regulation also become defaulters. So, these are the issues. (Senior Official 1, State Health Board) 
There was a perception among informants that the authorities responsible for overseeing regulations lacked the political will to enforce them. Generally, implementation of public policies in Nigeria is weak and the dual practice regulations are no exception. The private healthcare sector is a formidable interest group and it takes sheer determination from the government and civil service to implement policies that regulate its behaviour. The private sector gains extra strength from the cultural authority of the medical profession, which now is firmly opposed to any curtailment of DP in the country.

\subsubsection{Poor remuneration of doctors}

One of the senior policymakers working with the body that regulates medical practice mentioned the challenge of poor remuneration of doctors as an impediment to implementation. He explained that the general problem of low salaries for doctors working in the public sector is exacerbated by disparities in remuneration in hospitals at federal, state and local government levels.

What do we see today in our states? As I said, we have different levels, and they all pay different wages. Imagine a doctor at the local government level, for instance, earning the sum of N50,000 monthly (£105), then a doctor at the state level earning N100, 000 (£210) and that at the federal level going home with N150,000 (£315) monthly. You see the disparity; it could be that they have the same professional experience but earning different packages. So, many of them may not be happy; they may look elsewhere to make up. One of the challenges is the remuneration; government can see how to harmonize remuneration across the different levels. That might be a starting point to check some of the unhealthy practices among doctors. (Senior Administrator, Medical Council)

At many places in the interviews poor remuneration of doctors was mentioned as one of the main reasons for non-compliance with dual practice regulations. The drive for extra income may motivate doctors to engage in dual practice to make ends meet. Doctors at state and local government levels are among the least-well paid. Yet, even though they earn much less than doctors in federal government hospitals, there is no evidence that they are more likely to engage in dual practice.

\subsubsection{Weak sanctions for rule-breakers}

Respondents felt that even where rule breaking was detected by superiors, the sanctions for 
breaking dual practice regulations were weak.

The truth is that poor monitoring by the supervising agency is a big constraint to the implementation of dual practice policy, poor adherence by doctors themselves is a problem but enforcing discipline is the responsibility of the hospital management board to discipline erring medical practitioners. Do they do that? They do not. (Chair, House Committee)

There is a fear that the hospital management headed by the chief medical director who is a medical doctor may be lenient in applying sanctions against his colleagues who use government time for private practice. In some instances, those who are in-charge of punishing offenders might be offenders themselves. Take, for instance, the case of a medical director of a public hospital, who owns a nearby private hospital that operates twenty-four hours per day and is partly staffed by government doctors who probably do private work in government hours. These doctors may come late to the public hospital or leave earlier than necessary without sanction. The consultants who are in the management cadre in the public hospital may themselves use government time for their private practice. All these problems may weaken the impact of sanctions on doctors breaking dual practice rules.

\subsection{Policy of dual practice of medical professionals in Nigeria}

\subsection{Perspectives on mechanisms/options to manage DP in Nigeria and challenges for reform}

\subsubsection{Incentive approach}

Respondents were largely of the view that the motivation for dual practice was largely financial, and when they came to discuss how dual practice could be reduced many mentioned the possibility of using incentives of various kinds to achieve this. Under this theme respondents talked about monetary incentives, non-monetary incentives and challenges facing the incentives approach.

\subsubsection{Monetary incentives}

In the eyes of many respondents a simple way to use money to reduce dual practice was to increase public sector salaries. However, there was considerable disagreement about what 
levels of salary increase would be needed, and whether even with better salaries a certain proportion of DP doctors would still believe that private practice would still bring higher rewards. Some believed that salaries would need to be on a par with international standards before there could be a reduction of private practice.

That could be an approach because part of the yearning of doctors is to make ends meet. Sometimes the salary paid may not meet the needs. If our doctors can start getting what other doctors abroad receive, for example, in the western world, many of them would want to stay back after official work and play tennis in the evening, swim or relax than always trying to rush to private clinics. So, an incentive is also part of it. (Senior Official 1, State Health Board)

To be able to keep a doctor for ten hours in a public institution, you must be ready to pay him what is commensurate otherwise; he will look elsewhere to make up. The doctor is so important that he has to be where he is supposed to be at the right time, otherwise something can go wrong. We do not want a situation where a doctor will have one leg in and one leg out, patients will suffer for it. (Senior Administrator, Medical Council)

However, others have argued that salary increase may not stop dual practice.

It can help but will not stop it because peoples' want is insatiable. Once there is that gap, say, the salary is enhanced but there is no ban on DP, you still find some people who will continue to engage in the practice. So, if government wants to stop it let them raise remuneration and then ban it. The two things will have to go together. (Director, $\mathrm{MOH}$ )

Many believe that salary increase would without doubt discourage dual practice. They hold the opinion that good pay for doctors would help to keep them in government hospitals to complete their contractual hours. However, the point of disagreement between informants concerned just how much salary would be enough for the public-sector doctor to give up private practice. Some informants welcomed the idea of higher salaries but suggested that humans are insatiable and that such a move may not provide the solution to dual practice.

\subsubsection{Non-monetary incentives}

According to some the respondents' doctors might also be encouraged to remain working wholly in the public sector by non-monetary incentives, most notably by improving the working environment. This had a material dimension in terms of proper equipment and 
resources, but also the meanings that might be attached to public sector work, what is sometimes called the public service ethic.

The truth is that we belong to the school of thought that believes money does not really constitute an incentive. Pay people the correct salaries; it is their obligation. It is a responsibility. I will not call that an incentive. And then enrich the job that they do. That means that if somebody finds his job meaningful, if someone gets the right training that helps the job he does, and then somebody gets supportive supervision for the job he does. Those for me will constitute better incentives to make people work in the public sector. If you have the right tools and the availability of the resources that you need to work with, in terms of funding, if the facilities have overhead to run them, then these will constitute an incentive to keep workers in the public sector (Chair, House Committee).

It is not just about increasing money for doctors, if you do that they will just stay; also equip the hospital so that they can handle more patients. (Executive Officer 1, Nigeria Medical Association).

A good work environment would include the provision of equipment necessary for modern medical practice, as well as basics such as a constant power supply and proper patient care facilities. The mixed views of the study respondents suggested that doctors too might differ in the weight they gave to financial as opposed to non-financial incentives. While the former could make life more comfortable for the doctor, the latter might help to improve skills and enhance work performance.

\subsubsection{Challenges to the incentives approach}

Not all respondents believed that offering incentives would be an effective way of reducing dual practice. Two kinds of limitations were suggested in the interviews: the notion that incentives can create disharmony in the system, and the problem of offering either extra money or better facilities when resources are limited.

There was an opinion that incentives could create disharmony in the public system by provoking other professional groups to demand a pay rise. For example, if the government gives medical professionals a pay rise, other professional groups in healthcare or the wider public sector might request the same increase.

Of course, it will bring about disharmony in the health sector as it is currently going on. It will look as if you are trying to favour one healthcare professional 
group over the other. The nurses, the lab scientists, the pharmacists, radiographers, may query why preference is given to doctors (Senior Administrator, Medical Council).

A significant pay increase for one group might indeed result in wider disharmony, with a ripple effect across the public sector that would create conflict between government and other professional groups. Historically, pay has been a contentious policy issue between government and the public-sector doctors. The agitation for a pay rise has resulted in many industrial actions by the professional groups. Previous pay settlements granted to the doctors after acrimonious campaigns do not seem to have put an end to dissatisfaction with salary levels, and against the background of a falling Nigerian currency it seems that a step change that might stretch public finances too far would be needed to change this situation.

A major challenge for the Nigerian government at the present time is inadequate resources. The price of oil in the international market is sharply falling and Nigeria being a mono economy may find it difficult to either increase salaries or invest in the facilities needed to improve doctors' working conditions in public healthcare facilities. Several respondents maintained that incentives that carried an extra cost were unlikely to be feasible.

The issue of incentives is a challenging one. As a policy maker, I would be concerned because government resources are dwindling, and when you have dwindling resources, it will be a challenge especially if the focus is on financial incentives (Chair, House Committee)

I remember 15 years back, the private sector paid more than the public sector and because the private pays more, there was a lot of disharmony in the public sector. There are many things going wrong in the public sector, contracts for engagements are abused, frequent strike actions, demands for pay rise. Not all these will take place in the private sector. It will not be beneficial to government because it has to pay more. (Senior Administrator, Medical Council).

The economic situation of Nigeria is unlikely to improve in the immediate future, particularly with increasing use of alternative energy sources that may erode future oil revenues, so that there is little prospect that improved incentives will reduce dual practice. 


\subsubsection{Intramural approach}

Some healthcare systems allow doctors to treat private patients alongside patients receiving publicly-funded healthcare within the public facility, and this practice is being proposed in a few Nigerian public hospitals. This so-called intramural approach was mentioned by a number of respondents as a possible mechanism for managing dual practice. Four subthemes that came up in the interviews were: benefits of intramural to doctors, benefits to patients, benefits to the public facility, and challenges posed by intramural practice.

\subsubsection{Benefits of intramural practice to doctors}

Respondents outlined several benefits of the intramural approach to doctors in public hospitals. Among the claims made was that intramural practice raises doctors' income to a reasonable level, offers them the opportunity to use superior public hospital facilities to treat private patients, and enhances their skills development.

In some places it can be arranged that a consultant can have one or two additional beds where he puts his own private patients, and about 7 to 8 beds are for his regular work for the government, just in case someone rings him and says we want you to help us do this or that. It is still the same doctor, the same theatre. This is the area we should investigate. The doctors will support that idea because many doctors will like to have a second place where they work, they can tell their patients this is where I am. It is for the government to allow them to earn more through that platform... Some doctors drive across two or three cities to render services as per need. (Executive Officer 1, Nigeria Medical Association)

It improves the standard of practice and then the council has adopted the policy that a doctor should obtain and attain a minimum of 20 credit units - that is for continuous professional development (CPD). The doctor must participate in the $C P D$ or continuous medical education (CME) annually to obtain the annual practicing license. So, they must have a minimum of 20 CPD and each unit translates to one hour of CPD activity. That period of intramural practice will offer them a period of continuous medical education. It improves their standard and skills invariably. These are the two most beneficial factors that the practice will bring to the MDCN as the regulator of medical practice in the country. (Senior Administrator, Medical Council)

According to this informant CME works better in a setting where seniors are available to advice juniors, so that the team environment of the public hospital is more effective in improving standards than single handed practice in a private clinic. 
So, with this practice (the intramural), doctors will improve their standard of practice because when they practice within the public institution, which is also educational because we have hierarchies, from the consultant to the house officer. You find out that when a patient is brought to the hospital it is an opportunity for all cadres to learn from the patient's condition. It improves the standard of practice and then the council has adopted the policy that a doctor should obtain and attain a minimum of 20 credit units- that is the CME. (Senior Administrator, Medical Council)

Intramural practice allows doctors to earn extra income in the public facility where they work. The practice gives them opportunity to bring in private patients on a commercial fee-forservice basis and use public hospital facilities. However, the number of bed spaces allotted to doctors on private basis may pose challenges. For instance, if the extra income from intramural practice is not enough to compensate for giving up extramural practice they may revert to extramural practice that gives higher extra income. The cost to the government is also challenging. If there is low political commitment and ill-equipped hospitals, the situation may revert to the status quo.

\subsubsection{Benefits of intramural practice to patients}

At face value intramural practice means that patients who would have been treated in a private facility pay similar fees to be treated in a public facility alongside patients who pay only the more modest public hospital user charges. However, respondents claimed that fee-paying patients would gain advantages from this arrangement, particularly in the area of a reduced risk of medical negligence and malpractice by doctors.

Of course, for the Medical and Dental Council, having intramural practice would drastically reduce the number of cases of negligence and malpractice against patients that are reported to the Council. (Senior Administrator, Medical Council)

Respondents also suggested that public sector patients would benefit in terms of doctor availability during their contracted hours.

There may be a need to modify our approach to dual practice based on local realities by allowing a public practitioner to do their private practice in the public sector. By that, you can seize the practitioner entirely. For instance, if you are working for the hospital, you can bring in your private patients in the evenings or have a commission in some of the extra hours you may put in the public sector. In 
this way, nobody will be complaining about doctors' unavailability so that doctors can work as many hours as they would want (Executive Officer 1, NMA)

The benefit of intramural practice to patients was discussed and most informants agreed that it would ensure the availability of doctors in public hospitals. They stated that it has the potential to discourage doctors from working in two different places. They also described the practice as something that would reduce medical negligence and malpractice due to solo practice, which is common in private practice. This is because solo practice has no peer review mechanism; only the dual practitioner reviews a case and acts without input or advice from other colleagues. This approach, and the chance it gives to work in a team, would according to them reduce the risk to the patient.

\subsubsection{Benefits of intramural practice to public healthcare facilities}

Respondents mentioned three gains this practice might bring for public healthcare facilities: increased revenue, positive impacts on the public system, and the curbing of extramural practice among doctors.

Respondents argued that dual practice would increase revenue for government hospitals because they would receive payment for the private patients brought in by DP doctors. They also believed that intramural practice captures the doctor for the public system and therefore ensures that contracted hours are spent on hospital premises, with reduced movement between public hospital and private clinics.

Whereas if we are to practice intramural, if you bring in your private patients to the public facility, you use the public facility and pay for those public facilities, collect your own charges and pay the government its own (Senior administrator, $\mathrm{MOH})$

It is a workable approach. If you have a private patient, bring that patient to a government hospital, and then government will benefit because patients will be saved in the facility, and of course there will be part of the revenue going to the government and you will also have your own. Everybody will then be happy (Director, $\mathrm{MOH}$ ).

Apart from the increased patient-funded revenue, respondents argued that the intramural practice would also benefit the public system by reducing the diversion of patients away from 
public hospitals and incentivising doctors to work their contracted hours. Treating the majority of a doctor's patients in one location also brings benefits in terms of continuity of care and professional satisfaction.

If I finish any procedure here, I know the patient I operated on the previous day, I must go and check them, so if you have more than one place where you are doing that, you could have a divided attention, your mind will be here, and at a certain time you want to rush back to the other place. To limit all those movements, I think that was why the intramural practice was eventually introduced. I think the hospital should take that advantage and set it up very well so that doctors can admit their patients in the hospital and everybody stays in the same place. In that situation, there would not be any divided attention. The doctor will always be in the hospital attending to everybody even in emergency cases (Executive Officer 1, Nigeria Medical Association).

The other claim that some informants made was that intramural practice would curb the extent of dual practice by reducing doctor absences due to extramural practice.

Government can exploit a better way to utilise the doctors working under its employ. They can create the opportunity for their doctors for one or two hours of evening practice in government hospital for an extra income. That could make many doctors to do the so-called dual practice in one place. If they do it in one place, there will be less movement. It is that extra pay from dual practice that makes them to travel about with the risk involved (Executive Officer 1, Nigeria Medical Association).

Why we try to encourage intramural practice is that after work hours, which are 4 pm, you could still practice in your public health facility rather than go to a private health facility. So, we want you to practice here and earn some extra money while still earning your full salary in the public sector. (Senior Administrator, Medical Council).

The argument of these respondents can be summarised by saying that while in extramural practice doctors shift interest to their private practice, and the intramural system has the potential to keep doctors in the public facility. Those who have the intention to establish clinics for private practice can use the opportunity to make extra income in the same public hospital where they work. Such an approach could reduce dual practice and make the public hospital their only work place. 
There are acknowledged challenges to be overcome with any widespread introduction of the intramural including the issue of whether the number of bed spaces allocated for private cases is enough to make doctors give up extramural practice, and whether admissions to private beds is unfairly prioritised over admissions to public beds. There would still be moral hazard if doctors were able to pressure patients to choose private beds to secure early attention.

\subsubsection{Challenges of intramural practice}

The wider challenges that respondents highlighted were twofold: agreeing terms of reference for private practice in public hospitals and managing any unplanned increase in service utilisation.

Respondents were worried about defining the terms of reference for agreement between the public hospital and doctors for regulating intramural practice. These include setting the prices for private patients, allocating risk between the individual medical professional and the organisation, and agreeing a formula for sharing fees and costs between the doctor and the public facility.

The hospital fixes the price in terms of billing the patient but how much fee-forservice should the consultant receives per private patient needs be determined. The hospital might come out with what the admission fee will be, what the nursing fee will be, and whether whatever is charged to the patient goes to the consultant after paying the hospital its percentage. (Executive Officer 2, Nigeria Medical Association)

...there could also be an issue with the adopted sharing formula between the hospital and consultants in terms of who bears more of the risk; if these things are not well defined, they can affect negatively the overall framework of the intramural practice. (Senior Official 1 State Health Board)

The definition of terms of reference is very important for the operation of the intramural approach. The fear, however, is that this approach might be corrupted and abused if the guidelines setting up the practice are not well defined and accepted by all parties.

A second challenge mentioned by respondents concerned the likely increase in utilisation of the public hospital because of the introduction of the intramural approach. It was also noted that the intramural practice might not be feasible without the provision of upgraded 
equipment in the hospital. The challenge of inadequate facilities was a potential obstacle.

In most cases, some private facilities are more equipped than our public health facilities. That is why the practice has not taken off. For instance, you see that a public health facility does not have a CT scanner, but a private facility owned by a practitioner working in the public facility would have it. So, intramural practice may not take place in a public hospital that lacks basic facilities. In this case, it may not benefit the public health facility; rather, the reverse will be the case. (Senior Administrator, Medical Council)

Those things that may not make it to work out may just be some logistics like admission facilities. We do not have enough facilities for admission and things like that, and other facilities that patients may need. These things can bring some challenges to this option. Otherwise, if those things are there, it is a good idea. (Director, $\mathrm{MOH}$ )

The need to deal with an unplanned increase in facility utilisation seems to be a significant risk for the government. Procuring the necessary equipment needed in the new system remains a challenge. Despite the practicability of the new system, a lack of basic equipment and infrastructure could limit its operation.

\subsubsection{Doctors' implicit opposition to the intramural approach}

Respondents believe that doctors might oppose the intramural practice.

Government a number of times has tried to come up with the idea of accommodating doctors in the intramural practice, whereby government doctors can bring in their private patients to public hospitals and receive a fee-for-service. It has not taken-off the way it should, and our doctors are still engaged in private practice and denying people the services they should get during government working hours. Therefore, it is a major problem. (Director, $\mathrm{MOH}$ ).

That is what I have told you, that instead of private hospitals feeding the public hospitals; it is the reverse. They are now diverting patients from the public hospitals to the private ones. Whereas if we are to practice the intramural practice, if you bring in your private patients to the public facility, you use the public facility and pay for those facilities, collect your own charges and pay the government. (Senior Administrator, MOH).

Respondents fear that doctors might oppose the introduction of intramural practice. Efforts made in the past with doctors to debate and move towards the introduction of the intramural 
approach did not gain widespread support, particularly from dual practitioners. The degree of opposition from doctors encountered appears to have been sufficient to halt widespread implementation of this approach in the state.

\subsubsection{Allowing dual practice as the norm}

This section covers the topic coming up in many interviews of allowing dual practice as the norm. Related to this theme respondents talked of the danger to the public system, about how policymakers react to DP as the norm, and the challenges of allowing dual practice as what doctors routinely do.

In terms of dangers, respondents first, feared that unregulated dual practice would extend the market in private healthcare, and second, worried that dual practice aggravates the current near-crisis in the public system.

Respondents were of the view that unregulated dual practice will increase the size of the market for private healthcare. They suggested that such dual practice may lead to further multiplication of private clinics by government doctors and, in a bid to capture a sizable market share of patients, the diversion of public patient to the private sector might increase exponentially. The absence of regulation was already said to be leading to abuse of the public system, so that salaries were being collected for very little work.

It $[\mathrm{DP}]$ should be regulated. It would be abused if unregulated. Naturally, people will abuse it because they are sure their government wage will come, they will just go to their private settings and be making all the money, and every month they will collect their salary whether they do the work or not. (Executive Officer 2, Nigeria Medical Association)

Respondents also believed that unregulated dual practice would worsen the current situation in the under-resourced public system.

It will lead to a cascade of problem for all the stakeholders, including the patient. There will be total disarray as it is currently going on in our health system in Nigeria where doctors can use government time to go and practice in private health facilities. I remembered a state like X state where the governor frowns on $i t$. When he went to the state teaching hospital, doctors were not there, but when you go to their private facilities, they are there during government hours. Of course, that will not be good. (Senior Administrator, Medical Council) 
That sounds so deadly. The situation can be likened to an Igbo adage of asking a rat to help you keep a dry fish. Of course, you know what will happen. That illustration will tell you that it is no go area [laughter.] (Senior Official 1, State Heath Board)

According to respondents, this might lead to the withering away of public healthcare and a shift so that doctors focus entirely on private healthcare.

\subsubsection{Limiting types of services offered in the private sector}

The policy option of placing legal limits on the kinds of procedures that may be undertaken in the private sector is rarely discussed and did not gain much support from respondents in this study. Informants saw problems both in the notion of limiting types of services as acceptable way forward and practical difficulties in implementing such a policy.

\subsubsection{Negative effects of limiting types of services in the private sector}

Respondents said that limiting types of services offered in the private sector would unfairly disadvantage private hospital owners and full-time private practitioners who in many cases had an established track record of delivering many treatments that might be banned. They opined that such option might not be good for the private sector, which is a significant part of the Nigerian healthcare system.

No, you do not have to limit it. If someone decides to work in the private sector, why would you limit his or her practice? What could be done is that if this is your qualification, this is the limit of what you can do in the private sector rather than limiting it for every doctor irrespective of qualification and expertise. I think the limitation should be based on speciality. There are general cases a doctor should be able to do while more specialised cases could be limited to centres where you have specialists. If a hospital does not have a specialist, it should handle $X, Y, Z$. As I told you, some private hospitals are more equipped than the public ones. There are some private hospitals that train doctors even at postgraduate level. (Executive Officer 2, Nigeria Medical Association)

It sounds interesting, but again the risk there is over regulation because if I were a specialist in $O$ and $G$, and I choose to remain in full-time private practice, it will run contrary to good conscience for any government to do a policy that will not support my performing a CS or any gynaecological procedures in my practice. While it sounds a good option, I think it will be challenging to implement particularly in this developing and low resource setting. Let us look at care 
utilization, where do our people go for healthcare services, and why do they go to those places. There is more patronage of the private sector than the public sector, which suggests that there is some level of loss of trust in the public sector. Dual practice may be one of the contributing factors but certainly not all the factors. (Chair, House Committee)

Limiting the type of services provided privately may not be feasible in the Nigerian context because of the private sector's large role in the overall system, including medical education. As the public system is uneven in quality, with equipment shortages in some areas, a blanket ban on undertaking specified procedures in private facilities would cause considerable shortterm disturbance to Nigerian healthcare.

\subsubsection{Challenges in limiting types of services offered in the private sector}

Apart from the aforementioned problem, the challenges posed by limiting the services that can be offered in the private sector include implementing and monitoring the policy. According to informants such a policy additionally would not command wide support from those in authority.

If you were not monitoring them they would go out and do what they want to do. How would you know when they are doing it? That is the thing. It is only someone who is responsible, who thinks that the right thing should be done that can say I should not do this. Many people are just not like that. They feel that nobody should control them; they are free to do whatever they want. Otherwise, if people are self-disciplined, these things are very easy to do. Human beings are just something else. (Director, $\mathrm{MOH}$ )

The willpower of those in authority to implement to the letter the laid-down policy is lacking. We have this policy of intramural practice, but implementation has always been the problem. (Director, $\mathrm{MOH}$ )

Respondents catalogued a variety of drawbacks to this policy. Limiting the type of service offered in private practice may discourage the professionals who work in that sector. It may also affect future investment in the private sector. Poor monitoring of government policies remains a challenge and a lack of political will to implement the policy is another of the potential barriers. 


\subsubsection{Banning dual practice}

There has been debate over many years about the possibility of banning dual practice in Nigeria. Respondents in this study expressed a variety of views on this subject, which traversed various aspects of the issue. Among the topics on which they expressed opinions were: 1) conditions for banning 2) consequences of banning 3) policy makers' views on banning, and 4) opposition to banning.

\subsubsection{Conditions for banning}

For several of the informants banning would not be a feasible policy option until doctors' remuneration was addressed. Their view is that the present level of public sector medical pay is so poor that it would be unfair to doctors, and deeply damaging to their morale to cut off the possibility of additional private sector income.

The only thing doctors believe is that government is not paying them enough. If government will do that, it must pay them well so that the issue of dual practice ...That is just the issue. I know that could be a challenge. No matter how we try to check these things, if they are not paid well, they may also want to have a place where they can augment. That is the big problem. The government can ban it, but before a ban, it must think of how to beef up the remuneration of doctors and other health workers...like providing a car loan, housing loan and things like that. These are what will make people happy to work with commitment. These are what I think that government should be able to do. Giving a loan is not free money, the person that receives the loan pays gradually over a certain time and everybody is happy. (Director, $\mathrm{MOH}$ )

...we need to look at all those challenges that make a practitioner go for extramural practice. We must try to address those challenges. I did mention them such as remuneration, and the standard of health care facility have to be addressed. Once these are addressed, the practitioner has no reason to look outside. He will now look inside. (Senior Administrator, Medical Council)

As mentioned earlier in the chapter, the impact of a salary increase on dual practice is likely to depend on the percentage of pay uplift offered and whether such pay rises will be regular. If stretched public finances are unable to accommodate significantly higher pay for doctors then higher pay will not curtail dual practice, and in the eyes of many of the respondents government inability to raise pay also makes a direct ban on DP infeasible. 


\subsubsection{Consequences of banning dual practice in Nigeria}

Respondents foresaw two negative consequences if dual practice was banned. One is a further reduction in the availability of experienced specialist doctors in Nigeria, and the other is damage to the wider healthcare system.

The limited numbers of specialists in the public system is already a major problem in the Nigerian healthcare system, and respondents feared that a ban on DP would drive some specialists to the private sector or to seek overseas appointments.

In our own setting, we do not have the abundance of specialists, if you ban it; you may notice that some of the indices we monitor - infant mortality, maternal mortality, these diseases will deteriorate in Nigeria. It has been banned as I told you by the military and they saw that it was not beneficial to our system. It was repealed and now allowed only after the official contracted hours. Nigeria has trained less than three thousand specialists serving about 170 million Nigerians. (Executive Officer 2, Nigeria Medical Association)

Yes, we are short of specialists. We do not have that level of workforce to handle the number of the population we have in terms of specialists. By specialists, I mean the surgeons, pathologists, etc. You may go to a whole state and probably they have only one. Pathology is an issue because the public needs them especially up North where they are few, unlike the south, due to security challenges. Nobody will like to move up north. In that kind of environment, if you ban extramural practice, it could be an issue. That is one area to be investigated, you must look at the patient, as the centre of all these, are they going to get the care. So, trying to ban extramural practice and encourage intramural practice in some settings may not be ideal. From statistics available to the Council, we have few specialists compared with generalists, especially in critical areas like $O$ and $G$ where we have a lot of births. And of course, you know about the mortality rate that is quite high in the country because we do not have that level of manpower to take care of our women. The women are left to professionals in the lower cadre and when complication arises, the doctors are not available so, you do not want to ban extramural practice. (Senior Administrator, Medical Council)

Due to the variability of healthcare infrastructure and staffing in a nation occupying a large land area, and with an unevenly distributed population, any further loss of specialists from the public system may well compromise the government's ability to offer the full range of specialist services across the country. Given that poorer people can access private specialist 
services only with great difficulty, and perhaps not at all, the potential loss of specialists from the public system was seen by stakeholders as a critical issue.

Informants also predicted that a ban would cause wider damage to the healthcare system. One possibility mentioned was that dual practice might go underground and thus be even more difficult to regulate.

You know, I am one of those people that believe that if you want to bring in a change, it should be one thing at a time. A ban for me is very harsh especially in our environment. When you ban it, it might be more debilitating and worse than allowing it because people might be doing it without you knowing. It might lead to people doing what you cannot assess and then not able to curtail their practices and all that. (Senior Official 1, State Health Board)

Another prediction was that the links between doctors in full-time private practice and senior consultants or specialists in public hospitals would be weakened with deleterious consequences for clinical outcomes.

You may do that and make them to stay in the government hospital; I am more bothered about what will be happening to patients out there as not everybody goes to the public hospital. In the private hospital, expertise is scarce and if you have one doctor in the private hospital, that person will claim expertise of all areas of medicine; they cannot be surgeon, gynaecologist, paediatrician, etc. at the same time. We all have that general qualification but there will be a time of difficult cases that you will need someone who is specialised to come and assist. Yes, they can increase the money and restrict doctors practising in the public hospital but that could mean robbing them of their expertise and restricting the help they render to patients in the private sector. (Executive Officer 2, Nigeria Medical Association).

Any political move to ban dual practice would need to take account of the potential difficulties mentioned by respondents. Many returned to the point about the relatively high level of private practice in the Nigerian healthcare system. Given that over $70 \%$ of healthcare expenditure currently takes the form of out-of-pocket payments, changes in dual practice rules that may impact negatively on the private sector are likely to be approached with caution by politicians and policy makers. 


\subsubsection{Policy makers' views on banning dual practice}

Interestingly the policy makers in the study were divided into two opposed camps on this issue, either lining up against a ban or suggesting that a ban is realistic if dual practice were shown to be out of control.

There is a view from a section of the medical profession that a ban on dual practice would trespass on professional autonomy and would be contrary to the existing legal framework which defines the implicit agreement between the state and the doctors.

MDCN is a federal Act. Any law in any state that is contrary to the MDCN Act is, to that extent of inconsistency, null and void. Any laws that we are making at a state level is to give effect to the provisions of the Medical and Dental Council regulations. For instance, our regulatory framework must be consistent with the regulations made by the MDCN. So, what we do in terms of our regulatory policies, or even when we convert these policies to law is to substantiate, to domesticate and give effect to the provisions of the regulatory framework by the MDCN. The MDCN regulates the practice, we as employers now will say ok that doctors must come to work at 8 am and finish at 4pm and depending on the number of other doctors they work with, they can work twice or thrice in a week. So, the days you are not on call, we have no issues with you, and that is the consistency with the MDCN. (Chair, House Committee)

So, if a state decides to ban DP, it is going against the right of the doctor. It cannot say we are totally banning you from dual practice, what happens after office hours. I remember the time of the late Ransome Kuti [past Health Minister] that when this intramural /extramural practice regulation came into force in 1989 or thereabouts, doctors were given the privilege to work in private facilities after the closing hour. It is allowed for a doctor to earn some money after the closing hour but not during working hours. The state cannot ban dual practice as it is going against the rights of the doctor. However, a state can say, you should not use our time for your private work. (Senior Administrator, Medical Council)

The opposing group of policy makers believed that a ban on dual practice was entirely feasible if it was found to be out of control.

You know anything on health is on the concurrent list. That means that the state can legislate as it pleases about them. So, if you know what is good for you; you can do it. I do not think that MDCN is against a ban. If the state government says that this is what it wants you to do in the state, you must do it. Otherwise you are stopped from operating in the state. That is the issue. (Senior Administrator, $\mathrm{MOH})$. 
But I think that what the state government is doing at the end of the day may achieve a more lasting solution than what we were doing before. Outright ban, there is that pronouncement now. So, what is remaining is for us to get to the field, and very soon we will start the public service announcement that if you are involved in this, and government also attached a very stringent condition to it, that if you are caught you are dismissed on the spot. So, there is no question of giving a query. I think that when we hit the field it will give us a more lasting solution to the problem than what we are seeing now (Senior Administrator $\mathrm{MOH}$ ).

As I mentioned, government can stop DP. They can ban it if they find it difficult to control. But in banning it, they must also think of the interest of the doctors (Director, $\mathrm{MOH}$ ).

The final sentence in the above quotation points to the way that opinions on this issue may be entangled with interests, and particularly the strain that it introduces into relations between the state and the medical profession. On one side of the argument is the view that the state, with its democratic mandate, is entitled to set the terms on which medical professionals' practice, and as the employer of hospital doctors has a right to introduce changes that encourage them to work their contracted hours. The pro-ban stakeholders held strong views that doctors were not performing optimally in the public sector due to conflict of interest arising from private practice. On the other side is the profession's view that the existing legislative framework reflects an historical bargain between the state and the medical profession that grants the latter a high degree of autonomy in return for working under difficult conditions for modest salaries. They argue that it is therefore, unfair to prohibit work outside the public sector in what might be construed as a restraint of trade. This conflict between the public employers' interests and the doctors' interests plays out across a range of policies in the Nigerian healthcare system. It is an aspect of the unending political struggle between the state and medical professionals.

\subsection{Chapter summary/conclusion}

In this chapter, the motives for dual practice were examined using different categories of respondents. The outcome of the ranking of motives for DP by dual practitioners shows that income enhancement scored highest followed by the prestige and fringe benefits in the public system. Other motives such as professional development scored high among motives for 
engaging in DP. Therefore, the findings show that income enhancement is the major reason for dual practice among public-sector doctors besides others. Similarly, the FGD groups pointed out that financial gain and organizational shortcomings are the forces influencing the motives for dual practice among government doctors.

On the part of government-employed doctors, they overwhelmingly agreed that bureaucracy and civil service rules in the public sector motivate them to engage in private work besides, poor salary and poor working conditions. On the other hand, policy makers/stakeholders perceived bureaucracy in government hospitals, economic pressure and poor conditions of work as drivers of DP. Reducing the bureaucracy in government hospitals and improving working conditions in the public system were upheld by the doctors as what could make them give up dual practice, although, some were afraid that government might not be able make this happen.

It was investigated that non-dual practice doctors did not engage in dual practice for several reasons. They wanted to avoid stress associated with DP. Some felt they could not cope with doing two jobs simultaneously. Also, family issue was cited as barrier to engaging in DP. Doctors who were still in their earlier career development avoided DP to allow them concentrate in their training. What we do not know is whether this category of doctors would relapse into dual practice if the barriers holding them are over.

The challenges in combating dual practice in Nigeria were regarded as corruption, lack of will to enforce rules against DP, in addition to poor funding and logistics. Our study also shows that weak sanctions for rule breaking was noted as problematic because some rule breakers can easily go off the hook due to their connections with influential figures in the society.

The study found different mechanisms that could be used to manage dual practice in Nigeria, with the intramural approach as the most feasible. 


\section{Discussion}

\section{$9.1 \quad$ Introduction}

This chapter revisits the research questions that guided this study and considers the answers that emerge from the various study components (the FGDs, IDIs, and HH survey), and how they relate to previous studies. Then the main empirical and theoretical contributions of the thesis are examined to see what new knowledge has emerged. Areas for future research are noted. Certain limitations of the study are discussed. In the concluding section the author argues that the study findings have significant implications for policy makers, hospital managers, and regulators of medical practice in Nigeria.

\subsubsection{Revisiting the research questions in this study}

The overall aim of the research was to investigate DP by medical professionals working in public healthcare facilities in Nigeria, and their motivations and attitudes towards it. This was achieved by addressing several more specific research questions.

1. What are the benefits and disbenefits of DP in the health sector in Nigeria?

2. What is the extent of physician self-referral in public hospitals in Nigeria?

3. How does the perceived quality of healthcare in the public sector compare with that of healthcare in private healthcare facilities managed by dual practitioners?

4. What are the motives that lead Nigerian doctors to engage in DP, or refrain from doing so?

5. What are stakeholders' views on the implementation of existing regulations on DP, alternative regulatory mechanisms/options for controlling dual practice and the challenges for reform?

The findings relating to each of these research questions will be discussed in turn. 


\subsection{Overall findings of the thesis}

\subsection{Research question 1a: What are the benefits of DP in the health sector in Nigeria?}

All three components of the study - the FGDs, IDIs and HH survey - bear on this question and they provide slightly different, though overlapping results. The focus groups concentrated more on the benefits of DP from the standpoint of service users and responses centred on the three areas of the availability of payment by instalments in the private sector, a better doctor/patient relationship and the near-immediate availability of DP doctors in their private clinics. The results from the HH survey show similar benefits in areas such as less bureaucracy in private practice, speedier attention and better rapport of patients with DP doctors in private practice.

Three major sub-themes emerged from the IDIs, which focuses more on benefits from the perspectives of physicians. Doctors saw the major benefits of DP to themselves as coming in the two areas of extra income and professional development. Many claimed that there were also benefits to the public system in the two areas of reducing pressure on public hospitals and generating revenue for government. In their view, patients benefitted in the three respects of avoiding public-sector bureaucracy, establishing a better doctor/patient relationship and gaining quicker and better attention in the private sector. The findings from the focus groups, HH survey and IDIs, reflecting the respective views of service users and doctors, seem broadly consistent. Interestingly, the advantage of easier instalment payments was only mentioned by service users in the focus groups, and it was doctors more than patients who claimed benefits to the public system. Overall, data from these sources are arranged under seven themes.

\subsubsection{Speedier attention to private patients}

Private practice may offer improved access to healthcare for many reasons. The service user does not have to go through many levels of administrative 'checkpoints' as in the public sector. Instead, they have a direct access to the DP. Again, service users who go private are not subject to rules and regulations that apply in public hospitals and escape the delays that 
these can cause. Previous studies in other countries have shown how private practice improves access to healthcare for some groups (Gruen et al., 2002; Berman \& Cuizon 2004; García-Prado \& González, 2011; Vujicic et al., 2011). As in this study, previous research has shown that DP physicians give an improved service to private patients (Garcia-Prado \& Gonzalez, 2007; Hipgrave \& Hort, 2013). Since private practice is a business, the DP physician has an interest in differentiating the product sold by giving priority attention to private patients.

\subsubsection{Availability of DP physicians in private practice}

It was found that DP physicians are available to see private patients on a near-immediate basis. Patients can generally be confident of seeing the DP doctor with minimal delay and certainly as the time of any appointment arranged; they have the sense that the practitioner is always at their service. This business-like model of attending to private patients first can be seen as a way of enabling private practice to survive in the competitive private market. As many other researchers have observed this easy availability of doctors to private patients comes at a cost to patients in public hospitals (Chisholm, 2000; Chaudhury, et al., 2006; McPake, Russo \& Tseng, 2014). Availability of physicians plays an important role in increasing patients' trust in healthcare, and the public system will only gain credibility in the eyes of service users when it too can provide timely access to doctors.

\subsubsection{Enhanced doctor/patient relationship in private practice}

Both doctors and service users suggested that a stronger doctor/patient relationship develops over time in private practice. Without the periodic changes in personnel likely in the public hospital, doctor and patient get to know each other, so that there is better continuity of care and the management of the patient is, to an extent, personalised or customised. The author was unable to find previous studies that explored the enhanced quality of private doctor/patient relationship in a developing country context. However, Strong's (1979) study of NHS consultations includes some comparative data on US private practice that highlights how the 'private' framing of the medical encounter puts the paying US patient on a more equal footing with the physician than in the 'bureaucratically' framed NHS context. Yates 
(2000) found that some NHS consultants devoted an excessive amount of time to private patients, mainly for the financial rewards.

\subsubsection{DP helps patients to avoid bureaucracy in the public sector}

Both service users and doctors said the bureaucracy and administrative rules and regulations of the public sector were problems for patients. Patients avoid bureaucracy because they value time. Private practice, therefore, provides an alternative for patients free from administrative hurdles (Brugha and Pritze-Aliassime, 2003). It may be appropriate to reduce bureaucracy in public hospitals so as to allow the system to be more responsive to patient demand.

\subsubsection{Reduction of pressure in public hospitals}

Doctors and, to a lesser extent, service users said that DP benefits the public system by reducing the numbers utilising services in government hospitals. Government hospitals may be better able to treat complex cases because they have a range of medical specialists. Many respondents claimed that DP can help decongest the public system by handling more routine cases for those who can afford to pay, while leaving free care or specialist services available to those who need them in public hospitals. In a typical public/private partnership both sectors are complementary (Berman \& Cuizon, 2004; García-Prado \& González, 2011), but arguably Nigeria can make improvements in this area.

\subsubsection{Paying in instalments for private practice}

The study found that DP physicians allow payment in instalments for private patients who cannot afford to make a one-off payment. Given that the majority of the Nigerian population lacks insurance coverage, paying for private treatment at best imposes a significant burden and be a catastrophic event. By offering an instalment payment option, many Nigerian DP doctors give poorer patients a way to gain access to private treatment, albeit by falling into debt. Public hospitals typically require up-front payment ('cash and carry'), except for the few who are covered by national health insurance. The existing literature has had little to say about instalment payments. However, a recent Nigerian study by Onah and Govender (2014) found similar payment arrangements in Southeast Nigeria. The availability of payment by instalments may enable a wider range of SES groups to access private care by reducing the 
up-front cost. However, it was unclear whether DP doctors charge an extra fee or an interest payment for using this option, and patients who are left in debt may suffer future problems.

\subsubsection{Extra income for dual practitioners}

Dual practice is beneficial to doctors as it allows them to augment public salaries, which most say are poor. Private income helps doctors cushion themselves from the effects of economic recession and public-sector cutbacks (Roenen, et al., 1997; Prakongsai, et al., 2003; Humphrey \& Russell, 2004; Akwataghibe, et al., 2014), and extra income was reported by respondents as a major reason for engaging in DP. This is important for DP policy reform as the design of incentive packages may be an important factor in managing DP. Future research is needed to compare financial and non-financial incentives and how they can influence DP physicians to give up DP for exclusive public-sector contracts.

\subsubsection{Professional development of DP doctors}

Doctor informants suggested that DP can help them develop their careers by providing experience of the wider case-mix of patients found in practice across the two sectors. In Nigeria, wealthy patients who can afford certain complex procedures rarely use public hospitals, so that such procedures may only be performed rarely in that sector. In developing countries with a weak public sector, experience gathered in the private sector could be transferred to the public sector and vice-versa (Berman \& Cuizon, 2004; Humphrey \& Russell, 2004; LaGrone, et al., 2017). There is a view that the public sector tends to hide weak physicians who are supported by clinical teamwork, and that these may struggle in private sector where individual decision making is critical. Thus, some argued that experience of both teamwork and autonomous practice would produce better rounded professionals.

Overall, the government's priority should be to regulate DP in such a way that the poor and the public system are protected. The use of well-designed positive and negative incentives may help to change doctors' behaviours. 


\subsection{Research question $1 \mathrm{~b}$ : What are the disbenefits of dual practice in the health sector in Nigeria?}

Alongside benefits, the three main study components yielded data on disbenefits. As with benefits a number of key themes emerged.

\subsubsection{Low commitment to public patients}

The flip side of the greater attention to private patients mentioned above, was low commitment to public patients. Many service users and doctors believed that a conflict of interest arises when time is shared between private and public work, so that the public sector suffers. Respondents suggested that public patients may be neglected, face delayed access and find that the time available for them to see and discuss with public doctors is short. Although a disciplined dual practitioner may be able to straddle both sectors, without one infringing on the other, this study found that there are generally detrimental effects on public hospitals and their patients. This replicates the findings of earlier studies (Gonzalez, 2005; Hipgrave and Hort 2013).

\subsubsection{Late reporting and absence of doctors from work}

Late reporting and absence of public doctors from work was found to be widespread. There seems to be limited commitment to public sector work, particularly with many senior doctors. Some may report to work on designated days, while days of absence may be spent in private work. Again, this is not without consequences in the public healthcare system, both in terms of loss of capacity and patient experience. Previous studies have shown that neglect of public duties by DPs is endemic in some developing countries (Chaudhury, et al., 2006; Ligia, 2014). The finding that the problem persists in Nigeria suggests that both hospital managers and the Ministry of Health need to strengthen their supervisory roles. The present neglect of the problem tacitly supports the flow of patients to private practice in search of timely attention.

\subsubsection{DP affects medical training in government hospitals}

Contrary to the claim that DP assists professional development, some informants believed it had a detrimental effect on training in government tertiary hospitals. The consultants who are 
responsible for training are not available in the public system, so that patients requiring specialist treatments be pushed towards private facilities owned by the public-sector specialist. Thus, non-routine cases that would have assisted residents in training, go instead to the private sector where little training takes place. Teaching hospitals are left with a narrower case-mix, leaving the residents with less than optimal exposure to the full range of cases that will be important in their speciality. It was difficult to trace any study that confirms or contradicts this finding, although many studies have documented a negative impact of DP on care quality in government hospitals (Ferrinho, et al., 1998; Berman \& Cuizon, 2004; Ferrinho, et al., 2004b; Muula \& Adams, 2006). Given the dearth of empirical studies on DP in Nigeria, this is another topic for future research.

\subsubsection{Sharp practice in the private sector}

The study found that DP often results in sharp practices regarding the care patients receive in the private sector. Most DP doctors engage in solo practice, resulting in a lack of peer review of what are generally autonomous decisions about the patient with no inputs from colleagues. They may potentially dabble in areas of medicine they are not trained in to gain greater financial rewards. For example, it is common for DP paediatricians to accept gynaecological cases even though their expertise in this field is limited. Another risk comes from the neglect of the standard operating procedures (SOPs) that guide care in public hospitals. Since the private work of DPs is not subject to review from senior colleagues; this can compromise patient safety (Hannoravonghai, et al., 2000; Mills, 2002). The continual expansion of the private sector makes it imperative to undertake further research on the nature and extent of sharp practices in the private sector.

\subsubsection{Impacts of dual practice in terms of the burden on patients' finances}

The study examined patient expenditures for treatment in private hospitals/clinics as a result of self-referral, and the estimated mean cost of treatment in a public hospital for the same conditions. Private treatment expenditure for the referral group following referrals from the public sector was examined, and then the estimated mean costs of treatment for this group had they been treated in the public system were calculated. Self-assessed financial impact of 
self-referral for the patient group referred was recorded, and the coping strategies used to pay for private treatment by this group was also investigated.

\subsubsection{Expenditures for treatment in the private hospitals when referred from the public system}

Although the literature suggests that private sector costs are higher than costs in the public sector, the author found no published sources that compared the actual cost of care in the private practice with care in the public system for a patient group referred from the public sector to the private sector. This present study sought to determine what private treatment costs patients when they transfer from the public system.

The total mean expenditure for treatment in private hospitals for all patients who were referred from the public hospitals was higher than if they had remained in public care at a cash figure of N32,104 (Nigerian naira) (Table 10). There was a patterned increase across all individual cost categories used in the analysis, so that private care always cost more than remaining in the public system. This gives rise to a suspicion that patient welfare considerations come second to profit, and that DPs are seeking to augment their public salary by charging a higher fee-for-service than would otherwise have been payable. Earlier studies support the notion that DP can drive up healthcare costs (Gruen, et al, 2002; Brekke \& Sorgad, 2007; González \& Macho-Stadler, 2013).

This study did not specifically investigate why care costs more in the private sector. It might be that the DP has high recurrent and capital costs to fund, for example, rent, supplies and equipment. In the private sector, prices are rarely standardised; most practitioners set their prices at their own discretion. Because private treatments cost more Government should consider using measures to discourage self-referral of patients from the public system.

\subsubsection{Estimated mean cost of treatment in public hospitals for the same health conditions for which patients were referred to the private sector}

The cost of treatment in the public sector was found to be comparatively low. The total mean cost for all cost categories was N9,960 (Table 11). The same pattern of low cost was noticed in the cost variables. This result shows how much it would have cost to receive public 
treatment had the patients not been referred to the private sector. The referred patients went to the private sector for the same health conditions they presented in the public sector, which makes it easier for a comparative treatment cost analysis of the two sectors. Previous evidence shows that DP can raise healthcare costs even in the public sector, as a doctor may over-treat to gain a reputation that assists their private practice (Socha \& Bech, 2011; González \& Macho-Stadler, 2013). In a typical NHS-type system, the system would bear the cost of overtreatment. Such incentives do not apply in Nigeria as patients rather than the system would bear the cost of overtreatment because the main payment mechanism is OOP, even in public hospitals. No evidence was found that high costs in the public system are attributable to DP. Nigerian public hospitals use a standard schedule of treatment prices that makes the cost of services affordable, especially for the poor.

\subsubsection{The extent of self-rated financial impact of private treatment for the referral group}

The cost of self-referral for the patient group may indicate that there are serious or very serious financial impacts for this group. Self-referral was found to have a greater financial impact for certain poorer individuals in the patient group, but numbers were so small that it would be unsafe to infer any clear pattern for either referral or the impact of the cost of private treatment across the referred group. The hint that there might be a greater impact on poor patients may well have arisen from the presence of a few high-cost cases. The author therefore suggests caution in generalising from the situation of a small number of individuals who reported problems of this kind to financial impact across the population. The existing literature is scant on evidence on self-rated financial impact of self-referral. However, other past studies have suggested that cost was inevitably higher when patients were self-referred (Mitchell \& Scot, 1991; Swedlow, et al., 1992).

\subsubsection{Coping strategies for paying for private treatment by the referral group}

The individuals in the patient group coped differently with paying for private treatment after self-referral. There were four coping strategies that were used in this study: borrowed money, sold household movable assets, sold family land and bill was reduced by the doctor. It is not surprising that people borrow to cope with paying for healthcare especially in a health system 
like Nigeria where healthcare financing is mostly out-of-pocket payment. It might be that some members of this group were cash-strapped at the time they were self-referred. This confirms Onah and Govender (2014) findings that borrowing was a common mechanism for coping with healthcare bills. One may speculate that borrowing become more necessary as costs rise, and a number in the sample had presented with complex conditions. There is need for a financial protection measure in Nigeria for people across all SES groups to help them to cope when they face a high healthcare cost.

A small number in this group sold household movable assets to pay private healthcare bills. In Southeast Nigeria, where this study was conducted, it is normal for people to sell household movable assets to fund medical bills. This mostly affected those who were poor and had more serious health conditions and were not covered by health insurance. The affected patients might not have any capital that can be converted to cash and whatever marketable assets were available could be sold for cash. In the context of DP, the literature is scant on self-reported coping mechanisms to pay for private treatment when patients are self-referred, and, therefore, it is not easy to compare this finding with data from previous studies. However, Onah and Govender (2014) also found that poor female-headed households had to sell household movable assets to meet the cost of healthcare for family members.

\subsubsection{Impact of dual practice in terms of value of hours lost to the public system due to absences by dual practitioners from normal routine}

This section examines the value of hours lost to the public system due to absences by DP doctors. For the purpose of estimating loss, the author estimated the impact of DP on a single federal tertiary hospital, which employs approximately seven hundred doctors spread across the grades of consultants, registrars, medical officers, and house officers. The number of these doctors involved in DP is not known as there are no official records of DPs in the State and Federal Ministries of Health. Taking account of salaries across a mix of grades, the author created several hypothetical scenarios to represent weekly absences from routine duties using various parameters; the same process was applied to on call hours (Chapter 2 Methodology). The estimated value of hours lost are discussed below. The discussion is based on worst and best-case scenarios in both normal routine hours and on call hours. 


\subsubsection{Best-case and worst-case scenarios}

For the best-case scenario, it was assumed that just 5 hours per week per DP doctor was lost. Taking one extreme of the range and assuming that all doctors across different grades engage in DP would involve 4.9 million naira $(\$ 16,013)$ (Chapter 5, Figure 5), which is the equivalent of an annual value of hours lost to DP. As the percentage of doctors engaged in DP falls so does the magnitude of the value of hours lost to the public system. If the percentage of DPs was reduced to $90 \%$ for instance, the corresponding value of hours lost were reduced to 4.4 million naira $(\$ 14,379)$ per year. Similarly, if only $80 \%$ of doctors engage in DP the value of hours missed to the public system were pruned to 3.9 million naira $(\$ 12,745)$.

Taking a worst-case of loss of twenty hours per week produces an annual value of hours lost equivalent to 19.4 million naira $(\$ 63,399)$ if $100 \%$ of the doctors in the hospital is involved in private practice (Figure 5). Even a notional cash loss of half this figure from 50\% engagement in DP is sufficient to have a substantial negative impact on patient care in the public system. The extreme case scenario discussed above highlights the large value of hours lost represented by government salaries paid for work that is never done, a loss that becomes very large if the pattern is replicated across the wider health care system. Even in the bestcase scenario, the Government may have been paying salaries for large volumes of work not rendered in the public sector.

The author found no previous studies that estimated the value of hours lost to the public sector of DP absences in public sector work. However, past studies have shown that health worker absences are common in developing countries (Chaudhury, et al., 2006; Ligia, 2014), and have also commented on transfer of resources from the public to private sector due to competition for time (Macq, et al., 2001; Ferrinho, et al., 2004b). The present study adds value by using sensitivity analysis to predict the value of hours lost under the different scenarios. It remains the responsibility of the government to reduce the outflow of resources from the public to private sector. One policy approach would be to design incentive packages that discourage DP and reward doctors' presence in the public system. Government may have to think about intramural practice that would allow doctors to engage in DP in the hospital where they work and encourage them to remain there for their contracted hours. 


\subsubsection{Impact of dual practice in terms of value of hours lost to the public system due to absences by dual practitioners from on call hours}

Apart from regular duty hours, doctors in government hospitals are required to be on call for certain periods, and the analysis was extended to consider the value of working hour losses arising from absences affecting this aspect of the work. Again, two scenarios are presented - a worst-case scenario and best-case scenario.

\subsubsection{Best-case and worst-case scenarios}

For the best-case scenario in relation to on-call duties in the selected tertiary care hospital a five-hour absence was again assumed. If $100 \%$ of hospital doctors were involved in DP. This would mean a notional value of hours lost equivalent to 1.8 million naira. If the number of doctors involved is reduced to $50 \%$, the value of hours lost to the selected public hospital from on-call hours would be reduced to 0.9 million naira $(\$ 2,941)$ per year. A further reduction of this number to $10 \%$, could reduce the value of hours lost to just a hundred thousand naira (\$327) a year.

Going to a worst-case scenario, a situation where ten on-call hours per week are lost due to absences while on call would result in a notional value of hours lost equivalent to 3.6 million naira (\$11765) (Figure 6). A 10-hour weekly loss due to absences across the full range of doctors' grades/levels is almost certain to reduce the early availability of expert assessment and could be especially detrimental to the quality of care in emergency medicine. This not only motivates patients to move to the private sector, but again represents a significant loss to the public hospital in terms of payment for work not undertaken. Problems in staffing on-call hours may lead such cover to be reduced and may incentivize paying patients to seek care outside the public system (Ferrinho, 1998).

\subsection{Research question 2: What is the extent of physician self-referral in public hospitals in Nigeria?}

This section deals with the findings that emerged from the survey instrument, focus groups and IDIs with policymakers and doctors, focusing on key themes. 


\subsubsection{Age group of patients referred from public hospitals}

In terms of who gets referred, age appears to be an important factor. Patients aged 60-80 years are on average the most likely to be referred followed by the 18-38 age group, although the result is not statistically significant $\mathrm{F}(\mathrm{P}<0.51)$. In Nigeria, many elderly persons do not visit the hospital except at a crisis point. The reasons for delaying a hospital visit can range from cost of care (especially for poor families) to poor service delivery, and many are not covered by any health insurance policy. Social care provision in Nigeria is minimal and does not cater for the elderly. Instead, older people depend on their social capital-family, relatives, or friends for their care needs. In times of crisis, immediate intervention is necessary but may not be available in the public system due to bureaucracy and delays, so treatment in a private facility may be the only option. This is the type of case most likely to be referred in public hospitals but whether it is based on patient welfare or the chance for opportunistic DPs to profit remains unknown. The investigator could find no empirical study that examined the age profile of diverted patients. Past studies discuss diversion of patients but without this level of detail (Biglaiser \& Ma, 2007; Garcia-Prado \& Gonzalez, 2011; Abera et.al, 2017).

\subsubsection{Proportion of male and female referred from public hospitals}

The study found that more males than females were diverted. This is in line with Nigerian cultural norms that cast males in more economically independent roles than females, and probably reflects a perception on the part of DP doctors that males will be better able to pay for private care. In Nigerian society women's ability to pay medical bills will in many cases be dependent on the support of a male partner or relative. Whatever the precise reason, this study found that more females (278) than males (129) attended a public hospital and more males (18) than females (16) were diverted to the private sector. A study of public-on-private dual practice in Ethiopia recorded a higher number of males than females admitted in zonal hospitals but did not show which gender was more likely to be referred (Abera et al., 2017). The result from the present study differs because it found that more females than males were attending public hospitals, with more males than females being diverted. 


\subsubsection{Direct diversion of patients}

Diversion of patients to the private sector can take different forms. The two major strategies were the doctor speaking to patients and advising them to go to a particular private hospital/clinic or making use of nurses to make a similar recommendation (Figure 9). Such interactions often involve negotiation with the patient and are usually couched as professional advice that in many cases may genuinely be in the interest of the patient. Patients believe that the doctors and nurses concerned are disinterested agents who have their interests in mind and will always protect them, but this is often not the case. The result of diversion may be that a patient who would have low cost treatment in a public facility now faces a hefty bill for private services, and in some cases that quality of care may be worse rather than better. Patient diversion has been reported in several different national settings (Abera et al., 2017; Garcia-Prado \& Gonzalez, 2011; Rokx et al, 2010). The diversion rate seems lower in this study than that found in the Ethiopian research (Abera et al., 2017). The referral rate in their study was $19.2 \%$, whereas the present study recorded $8.4 \%$.

\subsubsection{Indirect diversion of patients}

Indirect diversion of patients also takes different forms. Nurses could be used as middlemen to push patients in more subtle ways to visit the consultant's private practice. This is often cleverly done when the nurse indirectly prods the patient to take the consultant's telephone number and suggests they arrange to see him elsewhere as he might not be available for their next appointments in the hospital. Another form of indirect diversion is to make things difficult for patients in the public hospital so that some patients themselves ask if they can see the consultant in his/her private practice. By creating delays or hurdles in the public hospital, consultants and their staff can make patients seek private care even when they did not anticipate using it. At other times the mere non-availability of a sought-after consultant, who makes no effort to make himself accessible within the public sector will be enough to move some patients to ask if he can be seen in his private practice. Indirect diversion has not been described in detail in the literature on DP. As far as the author knows only one previous study touches briefly on the issue of how physician ownership of a facility could lead to indirect inducements that channelled patients towards using it (Mitchell \& Sass, 1995). In this 
instance, the DPs may be creating demand for their private care indirectly and at a higher cost for unsuspecting public patients.

\section{Research question 3: How does the perceived quality of healthcare in the public sector compare with quality of healthcare in private healthcare facilities managed by dual practitioners?}

The findings that emerged in this section were from a focus group with service users and Likert-type survey questions. Again, certain key themes emerged.

\subsection{Structure element of quality}

\subsubsection{Better infrastructure/ equipment and availability of drugs in public hospitals than in private practice}

Generally, government hospitals were perceived as having better infrastructure, equipment and drug availability than most private facilities. Most government facilities are housed in substantial permanent buildings in accessible locations. Unlike most private practices, government facilities are rarely located in a rented building. Some smaller private offices are set up in rented flats in residential buildings, and the physical setting can be less than ideal. For example, the waiting room may be too small to contain patients, while the seating provided may be inadequate for the numbers attending, resulting in some patients queuing outside while waiting for their turn to see the doctor. Items such as oxygen cylinder, suction machine or defibrillator may be missing in the treatment room. Although there are often also equipment deficiencies in government hospitals, high-level hospitals will normally have medical equipment of this kind.

Conversely some established consultant DP physicians have better equipment in their facilities than the lower-level public hospitals that are supposed to act as gatekeepers. This means that service users may be uncertain as to which of the sectors has better equipment and drug availability (Siddiqi, Hamid, Rafique, \& Ali, 2002). While many believe that government hospitals have better equipment, others hold the reverse view. The area where clear differences do emerge is the tertiary hospitals and certain specialist medical centres, 
which clearly do have more sophisticated equipment than even the larger DP doctor-owned hospitals.

Even though drug availability is generally better in public hospitals than in private practices, as found in this study, the overall picture is far from straightforward. The drug supply chain in government hospitals may be bureaucratized, leading to periodic shortages and delays in availability. In contrast the private sector is less affected by administrative hiccups and so may have better drugs supplies at certain times (Gilson, Alilio, \& Heggenhougen, 1994; Sima, Heywood, Sandy, \& Garner, 2011). In reality, the dual practitioner can ask for a supply of drugs from their pharmaceutical representatives at any time needed and supply may be made without much delay. The lower-level public hospitals may experience drug non-availability more than the tertiary level due to the supply chain management challenges that arise when managers at the higher level decide when to supply drugs to facilities at the lower level, although, the Drug Revolving Fund (DRF) could be used to counter drug shortages in some lower-level facilities where it operates.

\subsubsection{Extended business hours, but fewer qualified healthcare staff in private practice than in the public hospital}

The private sector offers extended business hours, but usually fewer qualified healthcare staff. The opening and closing times seem to favour service users. Private practitioners compete for business by providing flexible access at convenient times. This practice of extending business hours could mean serving more clients in need by bringing care closer to the people (Basu, et al., 2012). Public hospitals are run as civil service organizations with an OPD closing time of $2.00 \mathrm{pm}$, meaning that patients visiting public hospitals later than that can usually only see a doctor via the Emergency Unit. It has been suggested that the hours of availability of DP physicians may be greater in their private practice than in their public work place (Berman \& Cuizon, 2004). But while the extension of business hours may improve access for private patients, DP time spent away from the public hospital means worse access for patients relying on that service.

Poor staffing in private practice sometimes has a direct effect on clinical quality. To keep labour costs down many DP doctors employ only a few staff, perhaps in smaller office only a single unqualified nurse. Some of the clinics, depending on how busy they are, may have one 
trained nurse who doubles as pharmacy attendant and receptionist. Russell (2005) goes as far as to describe the typical private sector staff complement as an incompetent workforce. The public system insists on proper qualifications and meets all professional licensing requirements. Moreover, public doctors working in a clinical team are subject to nearcontinuous peer review. By contrast the widespread use of unqualified personnel in private facilities is reported to have resulted in poor diagnostic accuracy and sub-optimal medical management (Basu, et al., 2012).

\subsection{Process element of quality}

\subsubsection{Better rapport with doctors in private practice}

The findings from the process element of quality indicate that respondents believed there was a more positive relationship with the doctor in private practice, including in areas such as confidentiality. There is a perception that doctor and patient interact more closely, and that this leads to a better understanding of the patient's needs and to individually customized management. Doctors make care plans and guide patients through the system. In terms of Strong's (1979) analysis of the 'ceremonial order' of the clinic, DP physicians use the 'private' format, which has contrasting qualities to the 'bureaucratic' format widely found in public healthcare. There seems to be a difference in the style of selling private medicine compared with providing public medicine. The public system with its larger patient flows, standardized policies and bureaucratic procedures is less responsive to individual preferences and struggles to build the same kind of trust that patients say they have in their private physician.

\subsubsection{Less waiting time in private practice/ more time to explain things in a way patient could understand}

As explained in section 4.9.3 respondents reported that waiting time to be seen in private practice is shorter compared with the public system, largely because of the absence of bureaucracy. In private practice, the patient typically goes straight to a consultation with the physician. Given the fact that relatively few can afford private healthcare, there are rarely the long queues seen in the public system. The pattern of shorter waiting times in the private 
compared to the public sector was also reported in an earlier study (Basu, et al., 2012). In Nigeria, a private facility may be preferred among rural dwellers who need to travel for medical treatment for this reason (Brugha \& Pritze -Aliassime, 2003). Patients, therefore, may be more satisfied with the process element of quality in private practice than they are in the public sector.

\subsection{Outcome element}

\subsubsection{Better health outcomes in private practice}

The results from the Likert analysis suggest that private practice run by DP doctors achieve better outcomes than do public hospitals. Health outcomes may be regarded as the effects of healthcare received, but it is unclear whether service users are able to assess the technical quality of the treatments provided. Service users generally regard a quality treatment as one that produces a positive experience during the treatment process, while a negative experience may denote poor quality. A majority of respondents suggested that private practice offers better health outcomes in terms of quicker recovery and improvement in health status. Others might see good health outcomes as something not particular to private hospitals, but also available in public practice, having experienced both sectors.

Past studies show little agreement on which sector produces superior outcomes, either in terms of measurable clinical outcomes or patient perceptions of quality care. For example, Soeung, et al., (2008) report that outcomes in the public sector were generally superior, while Siddiqi, et al., (2002) found no consensus among service users as to which sector was best. The respondents in the present study come down on the side of the private sector providing better outcomes, even though many of them opt to use the public sector for such reasons as the availability of medical equipment and more qualified healthcare personnel. This means that a degree of ambivilance remains, and individual patients may make different judgements according to such factors as the nature of the condition to be treated and the ease with which specialist care can be accessed in their local healthcare facilities.

This comparison shows that in terms of respondents' perceptions, private healthcare is generally regarded as being better than public healthcare in respect of both the process and 
outcome elements of quality, while public practice does better in the structure element of quality.

\subsection{Research question 4: What are the motives that lead Nigerian doctors to engage in dual practice, or refrain from doing so?}

Data used to address this question came from the FGDs (service users), the IDIs (doctors and policy makers) and the ranking of motives (doctors). As well as investigating DP doctors' motives for engaging in DP, the research also examined non-DP doctors' reasons for nonengagement (Chapter 8). The following themes emerged.

\subsubsection{Motives for DP}

Income enhancement was ranked as the primary reason for DP. Government doctors use DP to support their public income. As reported in section 8.4.2 most DP doctors engage in private practice for extra income. This applies at all grade levels. Poor pay seems to be the major reason for private practice in many developing countries (Ferrinho et al., 1998; Humphrey et al., 2004; Jumpa, et al., 2007). However, even doctors in high income countries seek to top-up public salaries, so the situation found in Nigeria may not be simply a function of low salaries in a developing country, but rather the near-universal attraction of extra marginal income. Government policy on DP reform may need to take this into account.

Of course, DP is not just about private practice, but also why doctors choose to retain their public-sector employment alongside private work. Two related factors cited by respondents were the prestige and fringe benefits that come with work in public hospitals. Public employment brings doctors prestige and a sense of pride, especially if their hold a position in tertiary and specialist hospitals. DP doctors may use this prestige to advance their private work. Indeed, in many cases, the government appointment provided the platform to initiate DP, both in enhancing credibility with patients and providing a baseline income that reduced the personal risk associated with starting a business. Previous studies have shown that although public salary could be poor, it can grant credibility, prestige and job security for doctors (Roenen et al., 1997). 
The ranking of motives for DP shows that professional development ranks third. Doctors who work in both sectors are believed to have a well-rounded career experience, that is seen as an advantage for career progression (Chisholm, 2000; Heikkilä, et al., 2014). This may aid promotion or access to research funds in the public sector and create opportunities for those with entrepreneurial ambitions. In Nigeria, the private sector accounts for a larger share of the healthcare market than the public sector and owning one of the larger private facilities may itself be an important milestone in a successful medical career (Jamshidi \& Reilly, 2008).

\subsection{Perception of service users of doctors engaged in DP}

Service users believe that DP physicians engage in private practice mainly out of financial self-interest; it is seen as a practice that benefits the practitioner alone (Muula, 2006). There was a perception that commitment to public sector work has diminished in recent years, perhaps partly due to economic retrenchment and constraints on government expenditure. Many respondents saw DP in the context of a wider failure of the public welfare system. However, although there was a recognition that many doctors were pushed towards DP by poor salaries, there was also a view that the profession was too quick to engage in strike action that put its own interests before those of the general public.

\subsection{Doctors' views of private practice}

Doctors saw private practice as an escape route from bureaucracy in the public sector. Respondents suggested that apart from erecting hurdles to access for patients, the rules in government hospitals could affect doctors' clinical decision-making power and limit the range of treatments that could be authorised. Doctors value professional autonomy (Kankaanranta et al., 2007), and some feel that they are forced into a kind of civil servant role, in which their decision-making space is curtailed (Humphrey \& Russell, 2004). There were also complaints from respondents about poor working conditions in government hospitals, which has featured in previous studies and been described as a push-factor encouraging private practice (Hipgrave et al., 2013). Many respondents saw private practice as an arena that allowed greater autonomy and a more individualised treatment.

The policy makers/stakeholders also saw avoiding bureaucracy as a key motive for private 
work, followed by economic pressures, which mean that poorly paid doctors inevitably take this path. They also acknowledged that work conditions in government hospitals may affect doctors' performance. Several informants attributed the administrative challenges affecting the public sector to poor management. Mention was made of the lack of accountability for service delivery, and limited commitment to the public service ethic.

\subsubsection{What conditions could make a doctor work exclusively in the public system?}

Many of the DP physicians interviewed said that there were conditions that would lead them to give up their private work. Reducing bureaucracy and increasing physician autonomy were mentioned as among the most important. Some doctors said that improving the state of public facilities and the availability of necessary medical equipment might encourage them to opt for exclusive public-sector work. Although rarely mentioned first increasing doctors' remuneration came up in most interviews. What remained unclear though was whether salary increase in itself would remove the attraction of extra income. Past studies have not shown that increased salaries result in increased output by doctors (Gabric \& Lazic, 2013; Mossialos et al., 2005), and so it is unclear that this would result in cessation of DP.

\subsection{Reasons for an exclusive public work by non-dual practice physicians}

Some doctors may prefer to work exclusively within the public sector. This group of doctors may be regarded as mission-oriented public servants who place the public service ethos above self and have implicitly committed to support the public sector (Sochas \& Bech, 2012). A number of doctors interviewed claimed that they could manage with their existing public salary. Some in this group were critical of colleagues who perceived as being motivated by money rather than any sense of mission. Interestingly they in turn were criticized by DP colleagues who suggested that non-DP colleagues were generally the doctors who lacked necessary skills and experience to compete in the private sector.

Doctors' reasons for avoiding DP could be manifold, but generally involved a calculation that, overall, the cost of DP to personal life, wellbeing and career outweighed the financial 
benefits. Some doctors who had briefly tried private practice in the past had concluded that coping as they were was a better option than making the lifestyle changes and accepting the pressures that extra part-time work would entail. The commercial aspects of private practice were unattractive to some of this group. They preferred to avoid the stress involved in managing private clinics and pointed to the complications that might arise from moving between sites and juggling the competing demands of patients in the two sectors. Time spent on this curtails the time available for family, friends and other personal interests.

It was found that family commitments were the major reason most female doctors do not take up private work. Their maternal responsibilities such as daily child care, the school-run and involvement in their children's social activities, meant that few had time for DP. The cultural norms about the role of women in family life in Nigeria may result in a pattern where more men than women doctors undertake private practice. This may also show that noninvolvement in DP does not always indicate professional altruism but may depend on cultural and individual-level factors.

Despite what was said earlier about the career advantages that experience of DP may bring, many doctors who avoid DP take a contrary view and believe that DP may actually hinder early career progression. Career development in medicine may involve study, additional professional examinations, academic publications, engagement in activities such as seminars and conferences, and involvement in professional associations, and time spent on private practice may be a huge distraction. Some younger doctors prefer to spend off-duty hours studying and engaging in the activities necessary to secure progression to higher career grade. Studies in other national contexts suggest that career ambitions and professional development needs can cause doctors to limit their private practice (Heikkilä et al., 2014; Kankaanranta, 2007).

Overall it can be seen that any over-simple attempt to label DPs as knaves and non-DPs as knights (altruists) would ignore the multiplicity of motives present on both sides, and the fact that some doctors do genuinely perceive DP as a benefit to the healthcare system. 


\subsection{Research question 5: What are stakeholders' views on the implementation of existing regulations on DP, alternative regulatory mechanisms /options for controlling dual practice and the challenges for reform?}

In-depth interviews were used to explore this research question and the findings are discussed below.

\subsubsection{Stakeholders' views on challenges in combating dual practice in Nigeria}

Many of the stakeholders mentioned corruption as a factor that makes it difficult to regulate the worst aspects of DP. Corruption in the healthcare system takes many forms, some of which involve circumventing the rules on DP. A common tactic used by some doctors is to use connections to politically-influential people to block any disciplinary action. Top-down intervention from such actors undermines the position of administrators responsible for enforcing the rules. It results in a situation where some rule breakers are punished while others get off scot-free. Senior medical administrators such as chief executives and medical directors in the tertiary hospitals are in positions of authority to deal with rule breaking, but rarely seem to intervene to limit DP. There is a suspicion that professional norms that are tolerant of the practice extend to senior medical managers, who may themselves have financial interests in private facilities. The Nigerian healthcare system has not yet been affected by the kind of general management reforms common in the West, and the medical profession continues to have a dominant position, whether in policy making, service delivery, or supervision. In the area of DP, the profession does not yet appear ready to regulate itself.

Funding and logistical challenges were found to constitute additional impediments to curtailing DP. Against a background of constrained budgets, the supervision of government doctors by the overseeing central departments became problematic, as many facilities cannot be visited, especially the hard-to-reach areas. Consequently, the extent of truancy and absences is hard to determine. What looks worrisome is that the authorities do not seem to recognise the importance of enforcing this rule and neglecting it means shielding bad behaviour. 
A lack of will to enforce the rules limiting DP means that regulation is uneven and largely ineffective. Informants suggested that some organisational superiors who were responsible for policing dual practice regulations may themselves have been breaking the rules. The private sector is shielded by the cultural authority of the medical profession, which at the present time, is firmly opposed to any curtailment of DP. The private healthcare sector is a formidable interest group and it would take determination from the government and civil service to implement policies that regulate its behaviour. Those in charge of enforcement might have functioning private hospitals that run contrary to DP policy in Nigeria. Regulating DP when those involved in the enforcement of rules are themselves rule breakers remains a huge challenge to the system.

\subsubsection{Weak sanctions for rule-breakers}

It is worth returning to consider whether the weak sanction for rule-breaking doctors is related to the dominant position of the medical profession. In the Nigeria healthcare system medical professionals seem to be strongly in control of both policy making and service delivery. The different structures under the Ministry of Health are headed mostly by persons with a medical qualification. The Policy Development and Planning Directorate, Hospital Management Board, the State Health Board, the District Health Board and the Local Health Board are all headed by medical doctors. The members of the supervisory teams also consist of medics. The supervisory teams visit facilities in the state to ensure compliance with medical practice. Officials and senior doctors share a common background and may well be members of shared social networks. It would be unsurprising if this partially explains the leniency in applying sanctions against fellow professionals in DP who give excessive time to their private businesses. Some of the doctors in the management cadre may themselves own wellestablished private hospitals, which raises suspicions that those responsible for punishing offenders may be offenders themselves.

\subsubsection{Poor remuneration of doctors}

At face value, low Nigerian medical salaries make the successful regulation of DP difficult. Doctors who feel that their remuneration is inadequate may not readily comply with 
regulations. Especially in state and local government-run facilities, where salaries are lower than in federal hospitals, DP is commonly used to top-up incomes. Some respondents suggested that introducing a common medical pay scale across the three tiers of government would reduce the distributional imbalance between levels and provide a better foundation for subsequent reform.

\subsection{Perspectives on mechanisms/options to manage DP in Nigeria and challenges for reform}

\subsubsection{Incentive approach}

Other respondents argued for an immediate across the board salary increase. The use of an incentive approach, especially a salary increases, as way to reduce DP has been tried in several countries (Hanson \& Jack, 2010; Sæther, 2003), albeit with mixed results (Carr et al., 2011; Yang, 2006). If we are to believe respondents' accounts then improved pay would encourage some Nigeria doctors to cease DP, while for others, especially the senior doctors, the decision might depend largely on whether the government pay rise would equal the lost income from private practice. Other professional groups also have grievances about public sector pay and would regard a large rise of doctors as a sign of the undue influence of the medical profession. An attempt to give a pay rise to one group without others would be likely to lead to disaffection and industrial action by other professional groups. An across-the-board rise in public sector salaries is probably not feasible in the current situation, and the Nigeria public finances may not be ready to face the ripple effects a pay rise for doctors could trigger.

As an alternative, some respondents suggested managing DP by offering non-monetary incentives. This would involve improving the working conditions of doctors and providing equipment that can enhance doctors' performance. However, as with a pay rise, the government's restricted fiscal space is unlikely to permit significant investment in improved medical facilities.

\subsubsection{Intramural approach}

An intramural approach was commended by several respondents as a way of keeping doctors in public hospitals and gaining some revenue from their private patients treated in the 
premises. They argued that introducing the intramural approach would raise doctors' income to a reasonable level, while patients could choose between public or private care without leaving the public hospital. There is a reduced risk of negligence and malpractice for the patient as the doctor's work in teams as against solo practice in the traditional private practice, and, above all, the patient does not have to wait too long due to the non-availability of government doctors. The public system may benefit from this arrangement in the form of increased revenue and the curbing of extramural practice among doctors (Kiwanuka et al., 2011; Rickman \& McGuire, 1999). There seems to be fairly broad support among policy makers, practicing doctors, and other senior administrators, for this approach.

The present study found some challenges of intramural practice in Nigeria. The terms of reference that define the agreement between doctors and public hospitals may be problematic. The first issue concerns how the prices for private treatment would be set. This critically affects the income of the private practitioner and the degree of attractiveness of his part-time private work. However, the basic parameters of the intramural arrangement, including patient's charges and the sharing of fee and cost between the doctor and the public facility, would be likely to be determined by the host facility, which might be a source of conflict. A second issue concerns how well the government would be able to manage a resultant increase in facility utilisation that might be difficult to estimate in advance. Moreover, an intramural system attractive to patients might not function effectively without updating equipment and infrastructure to improve standards.

Most significantly many of the practising doctors interviewed opposed the intramural model. One important matter to be determined would be the medical grades allowed to operate intramurally in government hospitals. If the arrangement only allocates beds to consultants, then lower ranking doctors would probably continue with extramural practice but might in some cases feel unhappy about being excluded. Moreover, unless the income from intramural work was at least equal to past income from private outside work, many consultants who would be eligible to use public beds would be likely to opt out. 


\subsubsection{Allowing dual practice as the norm}

Allowing DP as the norm was rejected by the policy makers as an approach that would damage the public system. This is the scenario where the regulation of DP is abandoned, and doctors can take on private work without restriction. There is a fear that unregulated DP could further extend private healthcare in what might be termed as a 'mad rush' to capture a sizeable market share of patients. Respondents worried that unregulated DP would worsen the current situation in the public system.

Currently in Nigeria, DP seems to be outside the formal rules but implicitly permitted. The de facto tolerance of DP seems to be based on an acknowledgement that public salaries are poor and allows part-time private work as an avenue to make additional income (Ligia, 2014, Roenen et al., 1997). Although the authorities have no plans to liberalise DP further, they make no serious effort to stamp it out.

\subsubsection{Limiting types of services offered in the private sector}

The policy option of limiting type of services offered in the private sector, so as to limit its attractiveness to service users, was not supported by the policy makers interviewed. This option was opposed for a number of reasons. Limiting the type of procedures offered in the private market would affect the commercial viability of many private facilities and thus damage a sector that it important for the healthcare system, including for medical education. Such a restriction would affect future investment in the sector and have a detrimental effect on the morale of those who worked in it. Overall, the challenges in adopting this approach are many and there is little political support for such a policy. This approach is better suited to systems where the bulk of services are provided publicly and funded through social insurance, as, for example, is the case with the Canadian system (Flood \& Archibald, 2001).

\subsubsection{Banning}

A simple ban on dual practice was another policy option opposed by the stakeholders. Most regarded it as infeasible, given the problem of recruiting and retaining doctors on the present low public salaries and the difficulty of raising pay rates. Informants suggested that a ban would probably result in a brain drain of experienced specialist doctors, with damaging 
consequences for the wider healthcare system. It was argued that a ban would be likely to drive DP underground, where it would be even more difficult to control (compare: Bian et al., 2003). Several stakeholders were themselves medical professionals and concern was also expressed about encroachment on the professional autonomy of doctors, who believe that it is their legal right to practice medicine outside the government contracted hours. Because of the higher percentage of total healthcare spending going to the private sector in Nigeria, any policy change capable of affecting the private sector needs to be handled with caution.

There was disagreement among stakeholders regarding the power of the national government to ban DP. While some opposed a ban on the ground that it would violate the existing legislative bargain between the state and the medical profession, others were of the view that, since it is within the powers of the legislature to reform health policy at any time, the state has the power to ban DP if it is seen to be damaging the healthcare system.

\subsubsection{Limitations}

Like most doctoral studies that are carried out with limited resources this study has certain limitations.

First, in terms of the methodology, computed salary for the sensitivity analysis was based on gross pay and not the net pay. Although, the net pay would have produced a more precise estimate of the value of hours lost to DP, gross pay was used to avoid revealing the characteristics of the salary earners. One problem was that the net pay slip contains a scanned photograph of the employee, grade level and the amount earned, information which is considered confidential. The decision to rely on gross pay may have led the researcher to overestimate the value of hours lost compared with the value if net pay had been used. Similarly, the hourly-loss scenarios for doctors' absences used in this study were based on doctors' incomes estimated from the known salary scales for staff at different incremental points. They were not based on empirical data on the actual distribution of staff across the scale. This new salary structure, known as the Consolidated Medical Salary Structure (CONMESS), applies to all medical and dental officers employed in Federal health institutions in Nigeria. The salary structure includes specialist allowance, which applies only to medical and dental officers on grade level 5 and above who are employed as consultants. 
Other allowances include call-duty allowance, which is payable to medical/dental officers and earned only when an officer would have performed call-duty based on the existing call duty roaster. Additional allowances are the health professional non-clinical duty allowance, teaching allowance and hazard allowance, which are paid across-the-board to all medical and dental officers. Thus, the calculation of salary income received by a given doctor is complex and may change over time. To simplify matters the estimates of value of hours lost due to absences in the present study are based on a calculation of average salary across the points on the scale and not the actual salaries. The calculation also did not factor in the actual number of doctors engaged in DP, as such data do not exist. Instead, different percentage scenarios are presented. Replicating this exercise using actual salaries based on the real mix of grades in a hospital, and with accurate data on the proportion of doctors engaged in DP, would give a more solid basis for planning.

Second, the study did not include any respondents from rural areas, which makes the sample urban biased. Since rural dwellers also access the tertiary hospitals that featured in the study, it would have been interesting to find out if urban-based individuals were diverted more than those from rural areas. The tertiary hospitals are all located in the Enugu Urban area and they attract patients from both urban and rural areas in the state. Given the fact that some private hospitals and clinics are in rural areas, which are also owned by government doctors, understanding the rate of patient diversion in rural areas would be desirable. However, because of limited time and travel funding as well as the logistical problems of assembling focus groups of rural dwellers, this option was not pursued.

Third, allocation of respondents to the SES groups used for the study depends on self-reported status, which might have introduced social desirability bias and could make some respondents either over or under report their socio-economic status. There may well have been outliers in respect of the measures of household asset holding, living conditions and household weekly food consumption obtained from the household questionnaire that were used to identify socioeconomic status group within our survey sample. There was no way of verifying whether the weekly food consumption pattern of the household was what they claimed. For instance, those who live below the poverty line could report an increase in their weekly food expenditure to avoid being classified as among the poorest. 
Fourth, this study focused on one LGA, which was randomly selected. The information gathered in this study might have introduced location bias. If the study was done in another location that is either more deprived or better-off economically, it is possible that the extent of patient diversion and the way it is perceived may have been different. Theoretically, one location might have yielded limited information. For instance, the study LGA is the smallest with a land mass of $67 \mathrm{~km}^{2}$, and the least economically developed of the three LGAs. It has a population size of 259,000 based on a 2016 population projection. It also has many residential areas that are semi-urban but has no residential area considered as 'posh'. In contrast, the other LGAs are more densely populated. Enugu East, for example, has $383 \mathrm{~km}^{2}$ land mass and a population size of 374,100 with more of semi-urban than urban areas. It has only one affluent residential area where a relatively higher SES groups live. On the other hand, Enugu North has $106 \mathrm{~km}^{2}$ land mass and a population size of 326,900 . It has many well-developed and more affluent residential areas where higher socio-economic groups live. Potentially, there may be a higher concentration of private facilities in the larger and richer LGAs, and consequently, the rate of patient diversion may differ across the LGAs. According to Mitchell \& Scott (1992), their study shows higher utilisation of clinical laboratory tests, diagnostic imaging and physical therapy in affluent arears with a concentration of private facilities. Therefore, the rate of patient diversion may well depend on the population size of an LGA and the number of privately-owned facilities. Where there is a higher number of physicianowned facilities self-referral to private practice from public hospitals may be higher.

Fifth, the lack of existing literature and data on DP in the Nigerian context means that there are few points of comparison that this study can build on. In effect this is an exploratory study that examines an under-researched topic as far as DP in Nigeria is concerned. For instance, there are no reliable data on the number of DP doctors in government hospitals or the number of government doctors who own private facilities. The lack of information on the various aspects of DP means that presently the phenomenon is not well understood in Nigeria, so that research is urgently needed to inform policy.

Sixth, the residential areas in a typical urban centre may be differentiated by class differences and other socio-demographic characteristics. The two purposively selected residential areas were based on their accessibility for questionnaire administration. There were no other socio- 
demographics considered for their selection and the class-mix of people living in these areas may not be typical of other residential areas. For instance, people choose where they live based on income or status. This might have implication on hospital utilisation (private or public) as well as the rate of self-referral. In a place inhabited mainly by the poor, for example, the number of physician-owned facilities may be reduced and consequently selfreferral to private facilities may be minimal, after all, a DP will not self-refer a patient who has no ability to pay. However, this study did not take into consideration the social status differences that might exist across different residential areas and may suffer from clustering effects (see 2.5.4). This limitation poses a difficulty in generalising the result to other residential areas within the Enugu Urban area.

Seventh, the findings from this study may not be generalisable to other states in Nigeria. It is a case study of one state, which may not reflect the context and socio-demographics of other states. Given that the study collected no data from other Nigerian states, one cannot be sure that similar results will be found there. For example, every state in Nigeria has power to legislate over health matters, so it might not be feasible to use data from one state to apply to other states. The state ministries have devolved power to determine many aspects of local health policy and their monitoring and supervisory capacity varies. There is a future need for a multiple case study investigation across states to find out the general pattern of DP in Nigeria, which allows comparative analysis. In the same way, the results of the present study may not be generalisable to other types of district and secondary care hospitals. This level of care may offer services for specialities such as general medicine, general surgery, paediatrics, obstetrics and gynaecology as well as general community health services. Therefore, the basis for generalising of results from tertiary seems quite limited as the administrative structure, level of personnel, rate of utilisation, and the mandate of each level of care differs considerably.

Eighth, the study relied on patient perceptions of healthcare quality. The technical quality of healthcare was not measured directly in the quality assessment, and that could bias the study findings. It would have been very difficult to arrange measurement of technical quality across the diverse range of health conditions presented by the respondent patients. Self-reported 
outcomes were a pragmatic alternative but introduces a degree of subjectivity linked to personal opinions and different degrees of understanding of medical knowledge. This introduces some uncertainty about the robustness of the comparison of treatment outcomes in the public and private sectors.

Ninth, the number of patients diverted to the private sector was small in the study area $(n=34)$. There must be doubt about whether this under-report the true extent of patient diversion in the Enugu Urban area. An assessment of sub-group data from different residential areas in the Urban area could generate more accurate and reliable results on the extent of patient diversion.

Tenth, recall bias is a major issue in studies that rely on self-reporting. When people remember past events, they do not always give an accurate or complete picture of what happened. In this study, patients' recall of cost of treatment in private hospital up to one year before and their estimates of what this would have cost them in the public sector must be treated with some caution. In a future study, the recall period could be reduced to limit the extent of recall bias and increase the accuracy of treatment costs estimates. The use of real records data on treatment given and its cost of treatment would be more reliable, but almost impossible to obtain in a study with such a diverse respondent sample.

\subsection{Main contributions of the thesis to knowledge}

In outline, this study uses triangulated data from focus groups, in-depth interviews, HH survey, ranking and sensitivity analysis to shed light on the under-research area of dual practice in Nigeria and the policy discourse surrounding it.

One novel aspect of the research is the use of sensitivity analysis, comparing multiple scenarios, to highlight the value of hours lost to the public system of absences and truancy by DP doctors. A similar large flow of resources out of public healthcare was reported in Macq and colleagues (2001) study. However, the present analysis goes further by quantifying the value of paid hours lost to the public system in cash terms and this serves to emphasize the magnitude of the problem that DP represents. Measuring the cost in this way could make a 
valuable contribution to the policy discourse on DP, particularly in developing countries. This innovative method is a tool that policy makers could use. By estimating the number of hours lost to absences under different scenarios, government can then set itself a target to reduce the number of doctors engaged in DP, and over time reduce the number of hours lost and the consequent loss of value to the public system.

Similarly, the analysis of Likert items from the HH survey helped to generate an important insight by unpacking the elements that make up quality of care using the Donabedian threepath model of quality of care. Using this model, the study compares the quality of care in the government sector with that offered by private providers, in terms of structure, process and outcomes. It established that the public system has better structure (i.e. physical) element of quality than the private sector, whereas the process and outcome elements are seen as being better in private practice. Much of the existing literature suggests that quality of healthcare in developing countries is higher in private facilities than in the public system (Sima, Heywood, Sandy, \& Garner, 2011) and the present study found that — apart from the structure element of care - the private system was rated higher for both the process and outcome elements of quality.

Patient diversion seems to be common in healthcare systems where DP exists, whether in developed (Humphrey \& Russell, 2004) or developing (Abera, et al. 2017) countries. However, the literature on this topic tells us little about exactly how patients are diverted to private practice. The present study found that a pattern of referral exists in Nigeria whereby older adults are more likely to be diverted than younger adults, and more males than females are diverted. This appears to be based mainly on DP doctors' perceptions of ability to pay but may also sometimes reflect the necessity for immediate intervention for the treatment of the elderly, which may not be readily available in the public system due to bureaucracy and delays. The study found that both direct and indirect forms of diversion exist, suggesting that this is an organized practice in which DP doctors and supporting nurses cooperate. Past work has identified a need for evidence on the prevalence and effects of DP on health providers, consumers and private service (Berman \& Cuizon, 2004). The present study has shown that DP doctors often encourage diversion using both direct and indirect tactics and makes a start in mapping out the pattern of diversion in Nigeria. 
Additionally, the author has demonstrated the importance of the tactic used by DP doctors of attracting patients by offering the option of payment in instalments, which contrast with the need for up-front payment in public hospitals. It might be argued that a flexible payment approach of this kind opens access to health care by making private care available to those who ordinarily could not afford it. However, the benefit of spreading payment over times must be balanced against the consequences of higher payments and indebtedness.

The study suggests that those non-DP doctors who opt exclusively for public work do so for varied reasons, not limited only to a strong public service ethic. The literature might encourage a perception that this group consists of mission-oriented public servants, but individual circumstances rather than altruism can be a decisive factor. Some have engaged in DP in the past and found it incompatible with their personal schedules, career development or tolerance for the stress of running a business. For the female doctors, the family obligation of looking after children was often a major obstacle to DP. The study thus adds to the literature by highlighting the variety of motivations found when doctors avoid DP.

Moreover, the study provides new insights on how DP affects medical education in Nigeria. While supporters of DP argue otherwise, the respondents interviewed for this study pointed to clear negative impacts on medical training in tertiary hospitals. The informants point to how most of the trainers had moved their focus to private clinics and how informal peer review in clinical teams has been weakened. Even trainees spend much of their time in private settings, although without such team support. DP has created a situation where a sizable number of patients now prefer to patronize both the trainers and the trainees in their private practices, thereby diminishing the number of cases that could have been available for optimum training in the public sector. The study suggests that DP has highly negative implications for medical education in Nigeria.

While the relevance of the theory of professional dominance and power has been well documented around the world, this study provides further evidence of its applicability to Nigeria. It provides a case study for understanding the tacit agreement between the state and the medical profession. Drawing on this theory, we can see that the standing of a profession depends on the implicit bargain it has negotiated with the state, and the terms on which this 
allows it to exercise authority. The agreement between the state and the profession typically includes a 'regulatory bargain' that establishes the balance between self-regulation and regulation by external agencies (Stacey, 2000). In line with Freidson's (1970b) classic analysis, the Nigerian medical profession has remained as the dominant profession in the healthcare division of labour, exercising substantial control over the activities of cognate professions and substantially shaping policy in this field. Medically-qualified persons largely monopolize senior positions in the Federal and State Ministries of Health, as well as senior management positions in delivery organizations, which means that the profession is well placed to resist unwelcome policy changes. Indeed, it is argued that the reason some doctors leave clinical practice to become managers is not to collude to erode the profession's power, but to ensure that challenges to that power are resisted. In Nigeria, 'strata' of managementoriented doctors have taken over all major administrative and management positions in the healthcare system to the extent that they are in-charge at all levels. This suggests that attempts to increase compliance with the rules regulating DP in Nigeria are likely to be constrained by this pervasive professional power.

\subsection{Policy implication of findings}

In this section, specific policy implications of the findings for regulators of medical practice, Federal and State Ministries of Health, hospital managers, and public-sector patients are outlined.

The study findings have shown negative effects of DP on the public system, including financial losses through diversion of patients and value of hours lost through doctors' absences and truancy. DP affects the public system in such a way that training is compromised, and the quality of the trainees may be suboptimal. In the author's view this suggests a clear need for the Medical Council (the regulator of medical practice in Nigeria) to strengthen its capacity to monitor and sanction rule breaking, and more importantly to consider introducing additional incentives to wean doctors off DP. It is suggested that the use of incentives will have lower transaction costs than the legal restrictions that must go with monitoring (Kumaranayake, 1997). Different incentives designs may help to regulate the behaviour of doctors depending on what level of doctors are targeted. For instance, non- 
monetary incentives such as providing support (in terms of time and guidance) for career development might be preferred by some doctors to monetary incentives. The financial strength of the Directorate of Medical Services at the State level needs to be enhanced to collaborate effectively with the Medical Council. However, caution must be exercised in the use of financial as opposed to non-financial incentives to remedy DP. As mentioned earlier, this strategy has not been effective in other countries, and may be especially problematic in Nigeria because of current economic problems that include double-digit inflation and rising national debt. Currently many states in Nigeria find themselves unable to pay the minimum wage of N18000 (USD 59) per month. The current political uncertainty in the country is also challenging. The poor budget allocation to health is indicative of the poor commitment to the health sector. Successive governments have failed to match the 2001 pledge of the African Heads of State to allocate $15 \%$ of domestic budgets to health, let alone achieving the higher target of the recent 15\%+Campaign (APHA, 2010).

Furthermore, the Federal Ministry of Health, the employer of doctors at federal level and the State Ministry of Health at the state level can reduce the magnitude of the value of hours lost to the public system through closer monitoring of doctors' working practices, especially regarding the standard 40 hours per week contract. The sensitivity analysis shows that the value of lost hours to the public system is quite high, especially in a worst-case scenario, so that any reduction in the proportion of doctors working in DP represents a significant financial benefit to the system. This again suggests that devising incentives to keep doctors working mainly within the public system is a key area for policy development. The provision of the necessary attendance monitoring systems, necessary medical equipment and a workfriendly environment could enhance performance and boost attendance and punctuality. Furthermore, the appointment of hospital board members and CMDs of tertiary hospitals is frequently entangled with politics. In Nigeria, these appointments are often done to favour persons loyal to the ruling party, and especially those who are 'politically connected'. Such appointments do not result in a hospital board that properly represent its local community or members with the qualities needed for good governance. Boards appear ineffective in ensuring that doctors comply with rules and regulations governing DP. 
Hospital managers need to be alert to diversion of patients from the public system. The present study suggests that a significant minority of cases may be affected. An $8.4 \%$ of the sample was diverted by direct means to the private sector, and additional indirect diversions also took place. This aligns with other studies that found diversion of a significant proportion of patients (Garcia-Prado \& Gonzalez, 2011; Biglaiser \& Ma 2007). Diversion of patients is predatory behaviour, which is often presented in a manner that misleads patients. Although, it is technically illegal and an abuse of professional trust, the system seems to encourage it. Hospital managers find it difficult to eradicate patient diversion as those engaged in the act are the street level bureaucrats who by the nature of their work deal directly with patients. Since diversion takes place through a network involving doctors, nurses and perhaps the laboratory scientists, it is a shared secret and usually escapes the administrative radar. An alternative model that can be considered is to create a referral advisory committee in all units in the hospital. The role of this committee would be to ensure that any person who enters the hospital as a patient (from the GOPD) or begins an episode of care by arriving at an inpatient ward cannot be diverted at the consultant's discretion alone. Instead, there could be a joint review of an individual case between the consultant and the committee and if a referral is necessary then the committee can recommend a referral hospital where the patient will receive appropriate care.

This study found that doctors and nurses were the two common agents who instigated patient diversion. Their tactics are often direct but could also be subtle and devious. The implication of this for policy makers is that the welfare of public patients' needs to be safeguarded. Diversion increases the cost of care for service users and reduces the user fees flowing into the public system. Unnecessary referrals often occur because of a classic principal-agent problem associated with asymmetry of information between the health care provider and patient. For example, Chapter 6 (6.5) described the case of a diverted patient who was told by her GP that her case was too complex to be managed in the public hospital, thus necessitating her referral to the private practice of a doctor colleague. The patient accepted the referral without question and paid extra for the private treatment. This is the plight of many patients who trust the doctor to act on their behalf but find themselves paying a substantial amount for 
treatment that was available in the public hospital. The protection of patients is difficult to guarantee in a system where the power of the medical profession is overwhelming.

The present professional regulatory system in the shape of the MDCN Disciplinary panel appears to operate more to protect doctors than to safeguard patient interests. Currently, bureaucratic regulation of the healthcare system is centralised at the federal level, with the regulatory agency lacking sufficient resources to carry out its duties at the state and local government levels, and little effective oversight of DP policy. Government needs to expand regulatory capacity in the local government areas, with regulatory organisations comprising both doctors and non-doctors, and having a clear mandate to control DP. Importantly, public representative should be given a place in this expanded regulatory network, both through places on oversight committees and opportunities to provide information on experiences when investigations are undertaken by regulators. Suggestions for possible change by creating a new oversight organisation are put forward under 'Recommendations' in Chapter 10. 


\section{Conclusions and recommendations}

This thesis explored the DP of medical professionals who have full-time contracts in public hospitals and who also engage in private practice. This was achieved using a mixed-methods approach that employed a range of tools to generate knowledge about the benefits and disbenefits of DP in the health sector. The quantitative and qualitative methods helped to provide evidence on the perspectives of all the stakeholders about DP in the public sector in Nigeria. The research highlights the value of hours lost to the public system and shows how DP increases the cost of care for diverted patients. This work raises the important question of what the real contribution of DP to the public sector is and contends that the overall impact of DP on public system is negative rather than positive.

The evidence collected shows that DP brings benefits to service users mainly in the form of quicker access to private practice, and a better doctor/patient relationship. It can be argued that the availability of payment by instalments opens up access to care for those with insufficient funds to pay public hospital user fees upfront, but this must be balanced against higher prices and indebtedness.

Despite the benefits of DP for service users, the evidence from this study shows that the value of the resources flowing from the public system to private practices is substantial, particularly in respect of paid hours lost to the public sector when DP doctors do private work and lost fees that would have been paid to public hospitals if diversion did not occur.

The author argues that the pervasive presence of DP in Nigeria must be seen in the context of the long period of hegemony of the medical profession. The profession has achieved substantial cultural authority, economic power and political influence (Starr, 1982). It is the effective "controller" of the major institutions of the healthcare system, and its influence network extends into high-level federal and state political and civil service circles. This means that it has effectively 'captured' the system of professional regulation so that it is able to ensure that the rules on DP are not changed in ways that will disadvantage doctors. As a result, defaulters generally escape sanction and regulations are widely ignored. With the medical profession in such a dominant position, its members occupy almost all the key 
administrative and management positions in the healthcare system and are well placed to block unwelcome reforms. Many public hospital managers responsible for controlling and sanctioning DP, themselves have interests in private hospitals. This raises questions about whether the present system of professional self-regulation is working in the public interest, or if external regulation is required. The short-changing of the public system as a result of DP may be undermining the standing of the profession in the eyes of the public and leading to calls for greater accountability. However, as the stakeholders interviewed in the study indicated, it is not easy for politicians to hold the medical profession to account.

Patient diversion increases the cost of treatment for the diverted patients, so that for the poor, in particular, paying private treatment bills can be catastrophic, often necessitating borrowing, which may be difficult without collateral.

The evidence from this study shows that the quality of healthcare provided is not uniformly higher in public practice. Using Donabedian's three-quality elements, it was shown that the process and outcome quality elements actually scored higher in the private system than in public practice. Where public practice scored much higher than the private system was the structure aspect of quality - existence of better physical infrastructure, more qualified healthcare personnel, and a better availability of drugs. Private medicine could offer a more individualised form of care than the standardised approach of the public hospital.

It has been shown that the motives for dual practice are varied, but one common denominator is that most DP doctors seek an extra income to top-up their public salaries. This should not mask other genuine motives for engaging in dual practice such as professional development or skills utilisation but is generally there in the background. The financial motive seems to be common to DP physicians across different healthcare systems where DP is practised. However, the pursuit of extra income may not be a simple reflection of poor salaries. Since DP is practised even in countries with generous public sector pay (Humphrey \& Russell, 2004), it may be the attraction of marginal income at all levels rather than escape from a low income that motivates many. 
Moreover, the study found that public sector doctors who work exclusively for the public sector are not necessarily all mission-oriented public servants. Instead, they had various reasons based on individual-level factors as to why they could not do private work. These include personal reasons, such as career development ambitions or family commitments that lead them to avoid taking on part-time private practice.

Finally, among the different mechanisms and options proposed for managing DP in Nigeria, intramural practice appeared to command most support from policy makers and other senior stakeholders. They believed it to be feasible based on its potential to benefit the public system and to minimise absences due to the extramural practice. Additionally, intramural practice could help to reduce the revenue lost through patient diversion in the public system, and save patients from the risk of solo practice, which is characteristic of DP. The difficulty of pursuing this solution is that significant numbers of practising doctors, particularly those engaged in DP, oppose intramural practice, mainly because they perceive it would reduce their income and autonomy. Although this model exists in some European countries, how it would work in a national context where the regulation of extramural practice may be ineffective remains worrisome. However, despite the lack of support from front-line doctors this appears the most feasible of the available options for reducing the damage done to the public system by DP. The best chance of success would come if policy support for the expansion of intramural practice is accompanied by a framework of incentives that gives doctors at all grade levels a stake in intramurality, and rewards commitment to DP within public hospitals with improved opportunities for career development and collegial support. 


\subsection{Recommendations}

This study has generated new knowledge on the effects of dual practice in the public healthcare system through the exploration of the perspectives of key stakeholders. In light of the findings and other issues arising from the study, the following recommendations are put forward.

1. Public-sector doctors should be required to register details of any second place of work, and this mandatory registration information should be shared between their employing institution and the Federal and State Ministries of Health. This would improve on the current situation where the Federal and State Ministries do not keep any record of the extent of DP. Such data will assist in monitoring and policy development in this area. At present, knowledge of which doctors engage in DP depends on local knowledge of such things as which doctors run private clinics registered in their own names. Registration would be a prerequisite for approval to undertake private work while on a government contract. Accurate assessment of the extent of DP through such a register would help the planning and designing of incentive packages. Government hospitals could maintain regular communication with the private health facilities where their doctors undertake private work. This measure would help government hospitals to exercise better oversight of DPs during their contracted hours in the public sector. The fear would be that DPs may well collude with their private workplaces to provide inaccurate information on who engages in private work and for how long. There might also be strong opposition to this plan from a medical profession intent on protecting the interests of its members. However, the author believes that the Federal and State ministries of health must take a strong stance and push through meaningful change. This should include the introduction of penalties for any private workplace that hides information requested about a dual practitioner during routine checks. This is not so much a state intrusion into the affairs of private enterprises but as a reasonable measure to ensure that the full contract hours paid for by the public sector are utilised. 
2. Chief Medical Directors of hospitals should take steps to make patient diversion difficult. It should only be authorised officially by hospital management when such a referral is required for sound clinical or logistical reasons. A mechanism should be created to receive patient complaints in cases where they perceive that they are being pressured to transfer to private practice. This should include the possibility of complaining about nurses when they are involved in encouraging diversion. Although the CMDs - as managers of hospitals - have the power to enforce rules governing DP, it remains unclear how such rules can be enforced effectively when there is conflict of interest. For instance, several flourishing private hospitals and clinics in the Enugu Urban area belong to senior consultants on full-time public-sector contracts. The author believes that Hospital Management Board needs to control this conflict of interest by creating oversight bodies that take review of dubious practice out of the hands of individual CMDs and managers. Public hospitals should establish a referral advisory committee, which includes civil society and patient representatives, to work alongside the medical team in reviewing all cases meant for referral and sanction doctors who refer without consulting the advisory committee.

3. Given the enormity of the problem of doctors' non-availability in public hospitals as found in this study, an incentive that encourages physical presence on site should be put in place. Those who are able to achieve timely reporting and closing of work could receive a bonus at the end of the month paid as an attendance allowance alongside the main salary. Positive incentives may change behaviour where poorly enforced sanctions have failed. Many doctors hardly complete their weekly 40-hour contracts. They take advantage of the poor monitoring and supervision system in the Ministry of Health to shirk their public work. Even where monitoring and supervision are used regularly, some consultants have reached a status where they no longer obey rules governing their practice. This monitoring system must be strengthened, both in terms of stronger, top-down disciplinary action, and use of technology to record attendance, perhaps by utilising commercial time and attendance recording systems. Additionally, the public healthcare system could structure its promotion requirements to include among other things 'attendance reputation' achieved from early reporting and closing 
of work as a key performance indicator. Junior doctors seeking promotion would be required to achieve a certain number of recorded hours of work per week per year.

4. The government should discourage solo practices among dual practitioners. This is to avoid the sharp practices evidenced in DP due to the absence of peer review of clinical decisions made by DPs. This study shows that sharp practices among DPs are common and put patients at risk. DPs can then join in twos or threes to run a private practice. This is likely to improve patient safety by ensuring that difficult treatment decisions or unexpected complications are visible to other colleagues, whose advice can be sought, and mean that at least some team learning is available to juniors working in private facilities. Again, there would probably be opposition to such a change from medical professionals. However, the federal and state governments could offer certain incentives to make multi-doctor practices more attractive, for example, by offering material advantages to new start-ups through cheap land, soft loans, and free registration of business premises among others.

5. A process should be established to agree to the terms of reference for intramural practice between hospital management and the doctors in each tertiary hospital. This would include levels of private fees and bed space allocation and would be consolidated into a Memorandum of Understanding that would be subject to annual review. Necessary additional resources would be put in place by the hospital before the scheme started operation, and these requirements would also be subject to annual review. Admittedly, the potential challenges are many. For instance, reaching a mutually rewarding agreement on private fees and bed space allocation acceptable to both hospital management and doctors may not be easy. Splitting private fees in a fair way between consultants, senior registrars and other cadres would be a tricky matter. From the other side, hospital management will need to monitor arrangements closely to avoid any tendency to 'private bed first before public bed'. There is also the issue of the additional resources needed to upgrade the hospitals before the take-off of the scheme. The substantial financial investment needed to upgrade the infrastructure and procure equipment for the scheme, to make public hospitals attractive to private 
patients, could delay its operation, especially if rolled out across most tertiary hospitals at the same time. However, to overcome the financial constraint of a large roll out of the plan across the country, government could plan the scheme in phases. It could use a pilot hospital to assess how the scheme can operate and gradually extend it to other states to contain the financial costs associated with simultaneous implementation across the nation.

6. As mentioned in Chapter 9, there should be a way of protecting public patients from the predatory behaviour of doctors and nurses involved in patient diversion. The present regulatory system is ineffective. The MDCN Disciplinary Panel appears to subjugate patient interests to those of doctors. Furthermore, the introduction of the Service Compact (SERVICOM), a public service reform, by the federal government as a response to the poor service delivery practice in the public sector has not changed the culture of poor service delivery in the healthcare sector. In the author's view it would be advantageous to establish an independent "Health Watch" organisation comprising civil rights and patient groups, which would have a better chance of safeguarding patient rights. This group should neither be an appendage of the public sector nor under the control of the government hospitals. It should comprise a central umbrella organisation linked to a network of local Healthwatch organisations. These might link to oversight committees in larger health service units (hospitals). The involvement of public representatives alongside some professionals and managers within this new network would provide opportunities to feedback experiences when investigations are undertaken by regulators and create an accessible platform through which to lodge complaints. It would enhance the public 'voice' in the DP regulatory framework and influence how services are delivered and monitored in both the public and private sectors. It would counterbalance the power of the professionals to 'capture' the regulatory system and empower service users as the 'trusted guards" of their own healthcare. This 'bottom-up' approach would place medical practice under the public watch and help build trust in the health sector. 


\subsection{Postscript}

Following the 2016 Presidential election there was a peaceful transfer of power to a new president, Muhammadu Buhari, on May 29th. Shortly afterwards the Registrar of the Medical and Dental Council of Nigeria was replaced with a new appointed registrar for the Council. However, there is no evidence of any policy shift regarding dual practice in Nigeria from the time that the study started until the present.

\subsection{Future research}

This study has generated useful information for understanding dual practice in the Nigerian context. It has estimated the extent of patient diversion from the public sector to private practice and the resultant financial losses. The findings provide useful information about the impact of DP on the public system. Nevertheless, the research is subject to some limitations as indicated in Chapter 9.14.6. Some suggestions are therefore made for future research that would address these weaknesses.

This study presents a snapshot of the benefits and disbenefits of DP based on the views of service users, doctors and stakeholders, but benefits and disbenefits can also be measured using other methods and tools. Future studies using a range of methods would help determine how far the findings of this study apply more widely, and whether the combination of methods used in the present study misses dimensions picked up by other approaches. The central theme for future research should be who benefits from DP, and how it affects access to healthcare for the poor. The answer to these important research questions will help to clarify the benefits (if any) to the public system and public patients, particularly the poor.

Second, future studies should investigate the impact of DP on public facilities at the district health system level. It would be useful to explore the effects of DP at this lower level, which is in the domain of local health authorities. Most existing literature on DP describes activities at the central level. Research at the local level will show whether similar patterns are found there. 
Third, this study drew its sample from one location, so that its findings may not be generalizable to other areas. In effect it is a case study of one state rather than a survey that samples many locations across Nigeria. There is a role both for additional case studies that investigate whether the patterns found here hold across different settings, and across sectional survey studies that have a wider national focus.

Fourth, the present study used hypothetical scenarios of doctors' likely hours of absence to estimate the value of hours lost to the public system of DP. If a future study could obtain data on individual salaries rather than salary scales and the proportion of doctors engaging in DP, we could determine whether the approach used here is sufficiently accurate to be used as a management planning tool. Quantifying the value of doctor absences to the public system is important for planning purposes. The approach used here is relatively quick and easy to apply, but a detailed (and more expensive) study based on the real distribution of salaries between DP and non-DP doctors in a typical tertiary hospital would tell us whether the cheaper scenario approach is accurate enough to be useful. 


\section{REFERENCES}

Abera, G. G., Alemayehu, Y. K., \& Henry, J. (2017). Public-on-private dual practice among physicians in public hospitals of Tigray National Regional State, North Ethiopia: perspectives of physicians, patients and managers. BMC Health Services Ressearch 17(713).

Adeloye, D., David, R. A., Olaogun, A. A., Auta, A., Adesokan, A., Gadanya, M., . . . Iseolorunkanm, A. (2017). Health workforce and governance: the crisis in Nigeria. Human Reseources for Health, 15(32).

Adepoju, O., Opafunso, Z., \& Ajayi, M. (2018). Primary Health Care in South West Nigeria: Evaluating service quality and patients' satisfaction. African Journal of Science, Technology, Innovation and Development, 10(1).

Adesanya, T., Olayinka, G., Obadah, G., Marisa, M., Patel, B., Verma, R., \& Wong, H. (2012). Exploring the responsiveness of public and private hospitals in Lagos, Nigeria. Journal of Public Health Research 1(2).

Akinyemi, O., \& Atilola, O. (2012). Nigerian resident doctors on strike: Insights from and policy implications of job satisfaction among resident doctors in a Nigerian teaching hospital. Int J Health Plann Mgmt, 28, e46-e61.

Akwataghibe, N., Samaranayake, D., Lemiere, C., \& Dieleman, M. (2013). Assessing health workers' revenues and coping strategies in Nigeria - a mixed methods study. BMC Health Services Research, 13(387).

Alaref, J., Awwad, J., Araujo, E., Samira, L., Hillis, A., \& Özaltin, E. (2017). To Ban or Not to Ban? Regulating Dual Practice in Palestine. Health Systems and Reform, 3(1).

Alonge, O., Lin, S., Igusa, T., \& Peters, D. (2017). Improving health systems performance in low -and middle-income countries: a system dynamics model of the pay-forperformance initiative in Afghanistan. Health Policy And Planning, 32(10), 14171426.

Alubo, O. (1986). The political economy of doctors' strikes in Nigeria: a Marxist interpretation. Soc Sci Med., 22(4), 467-477.

Alubo, O. (1992). Health Services and Military Messianism in Nigeria (1983-1990). Journal of Social Development in Africa 7(1), 45-65.

Alubo, O. (2001). The promise and limit of private medicine: health policy dilemmas in Nigeria. Health Policy and Planning 16(3), 313-321.

Alubo, O., \& Hunduh, V. (2017). Medical Dominance and Resistance in Nigeria's Health Care System. International Journal of Health Services, 47(4), 778-798.

Anuro, W.B. (2014). Safeguarding human rights through combating Corruption, a research paper on the effects of corruption on human health. Retrieved from http://kenyalaw.org/kl/index.php?id=4542

Anyaehie, U. S. B., Nwakoby, B. A. N., Chikwendu, C., Dim, C. C., Uguru, N., Oluka, C. P. I., \& Ogugua, C. (2014). Constraints, Challenges and Prospects of Public -Private Partnership in Health Care delivey in a Developing Economy Annals of Medical \& Health Sciences Research, 4(1).

Arah, O. A., Gert , P. W., Jeremy, H., \& Niek, S. K. (2006). A conceptual framework for the OECD Health Care Quality Indicators Project. International Journal for Quality in Health Care; September 2006: pp. 5-13. 
Ashmore, J. (2013). Going private': a qualitative comparison of medical specialists' job satisfaction in the public and private sectors of South Africa. Human Reseources for Health 11(1).

Aveyard, H. (2014). Doing a Literature Review in Health and Social Care, a practical guide. NY: Open University Press.

Awofala, A. A., \& Ogundele, O. E. (2016). HIV epidemiology in Nigeria. Saudi Journal of Biological Sciences doi:https://doi.org/10.1016/j.sjbs.2016.03.006, 25(4), 697-703

Baah-Boateng, W., Adjei, P., \& Abena, D. O. (2013). Determinants of moonlighting in Ghana: an empirical investigation. African Review of Economics and Finance, 4 (2), 176-186.

Badejogbin, R. E. (2007). Law lecturers and private practice Review of Nigerian Law and Practice (Vol. 1, pp. 11). Lagos: Nigeria Law School, Lagos Campus.

Barnes, J., Chandani, T., \& Feeley, R. (2008). Nigeria private sector assessment : Private sector partnership-One project, Abt Associates Inc. Bethesda, 5-24.

Barnett, J., Barnett, P., \& Kearns, R. (1998). Declining professional dominance?: trends in the proletarianisation of primary care in New Zealand. Soc. Sci. Med., 46(2), 193-207.

Basu, S., J., A., Kishore, S., Panjabi, R., \& Stuckler, D. (2012). Comparative performance of private and public health systems in low and middle income countries: A systematic review PLoS Medicine, 9(6).

Berman, P., \& Cuizon, D. (2004). Multiple public-private job holding of health care providers in developing countries: An exploration of theory and evidence (pp. 6-36): British Government's Department for International Develpoment (DFID)

Bian, Y., Sun, Q., Jan, S., Yu, J., \& Meng, Q. (2003). Dual Practice by public health providers in Shandong and Sichuan Provinces, China Health Economics and Financing Programme working paper (pp. 1-36). London: LSHTM.

Biglaiser, G., \& Ma, C. A. (2007). Moonlighting: public service and private practice. The RAND Journal of Economics, 38(4), 1113-1133. doi: 10.1111/j.07416261.2007.00128.x

Blaauw, D., Erasmus, E., Pagaiya, N., Tangcharoensathein, V., Mullei, K., Mudhune, S., . . . Lagarded, M. (2010). Policy interventions that attract nurses to rural areas: A multicountry discrete choice experiment. Bull World Health Organ, 88, 350-356.

Blair, R. D., \& Rubin, S. (1980). Regulating the professions. USA: D.C. Heath and Company.

Bloom, G., Standing, H., \& Lloyd, R. (2008). Market, information asymmetry and health care: Towards new social contracts. Social Science and Medicine, 66, 2076-2087.

Bloor, K., Maynard, A., \& Freemantle, N. (2004). Variation in activity rates of consultant surgeons and the influence of reward structures in the English NHS. Journal of Health Services Research \& Policy 9(76).

Bowers, M. R., Swan, J. E., \& Koehler, W. F. (1994). What attributes determine quality and satisfaction with health acre services? Health Care Management Review 19(4).

Braun, V., \& Clarke, V. (2006). Using thematic analysis in psychology Quarterly Research in Psychology, 3, 77-101.

Brekke, K., \& Sørgard, L. (2007). Public versus private health care in a national health service. Health Economics, 16(6), 579-601.

Brinkerhoff, D., \& Bossert, T. (2008). Health Governance: Concepts, Experience, and Programming Options. Retrieved from 
https://www.hfgproject.org/health-governance-concepts-experience-programmingoptions/

Brook, R. H., McGlynn, E. A., \& Shekelle, P. G. (2000). Defining and measuring quality of care: a perspective from US researchers. International Journal for Quality in Health Care, 12(4), 281-295.

Bruce, J. (1990). Fundamental elements of the quality of care : A simple framework. Studies in Family Planning 21(2), 61-91.

Brugha, R., \& Pritze -Aliassime, S. (2003). Promoting safe motherhood throgh the private sector in low and middle income countries Bull World Health Organ, 81, 616-623.

Bryman, A. (2008). Social Research Methods. Oxford: Oxford Univsersity Press

Bustreo, F., Harding, A., \& Axelsson, H. (2003). Can devloping countries achieve adequate improvements in child health outcomes without engaging the private sector? . Bull World Health Organ, 81, 886-895.

Campbell, S. M., Roland, M. O., \& Buetow, S. A. (2000). Defining health care quality Soc Sci Med, 51, 1611-1625.

Carr, S., Leggatt-cook, C., Clarke, M., MacLachlan, M., Papola, T., Pais, J., . . Normand, C. (2011). What is the evidence of the impact of increasing salaries on improving the performance of public servants, including teachers, doctors/nurses, and mid-level occupations, in low- and middle-income countries: Is it time to give pay a chance? London: EPPI-Centre, Social Science Research Unit, Institute of Education, University of London, pp.1-19.

Chaudhury, N., Hammer, J., Kremer, M., Muralidharan, K., \& Rogers, H. F. (2006). Missing in action: teachers and health wokers absence in developing countries. Journal of Economics perspectives, 20(1), 91-116.

Cheng, T. C., Haisken-DeNew, J. P., \& Yong, J. (2015). Cream skimming and hospital transfers in a mixed public-private system. Social Science \& Medicine, 132, 156-164. doi:https://doi.org/10.1016/j.socscimed.2015.03.035

Chengsorn, N., Bloss, E., Anekvorapong, R., Anuwatnonthakate, A., \& Wattanaa-mornkiat, W. e. a. (2009). Tuberculosis services and treatment outcomes in private and public health care facilities in Thailand, 2004 - 2006 Int J Tuberc Lung Dis 13, 888-894.

Chisholm, C. D. (2000). The moonlighting paradox. Ameican Jounal of Emergency Medicine, $18(2), 224-226$.

Collier, R. (2012). Professionalism: The privilege and burden of self-regulation. Canadian Medical Association 184(14), 1559-1560.

Creswell, J. W. (2014). Research design: Qualitative, Quantitative \& Mixed Methods Approach. London: SAGE.

Creswell, J. W. (2015). A Concise Introduction to Mixed Methods Research. London: SAGE.

Cruess, S., \& Cruess, R. (2005). The Medical Profession and Self-Regulation: A Current Challenge. Ethics Journal of the American Medical Association, 7(4).

Das, J., Holla, A., Mohpal, A.(2016). Quality and Accountability in Health Care Delivery: Audit-Study Evidence from Primary Care in India. American Economic Review, 106(12):3765-3799.

Davies, H. T. O., \& Crombie, I. K. (1995). Assessing the quality of care. Br. Med. J., , 311, 766. 
Davies, M. (2014). The future of medical self-regulation in the United Kingdom Renegotiating the state-profession bargain? . Medical Law International, 14 (4), 236265.

Denscombe, M. (2014). The Good Research guide (Fifth ed.). England: Open University Press

Diara, B. C. D., \& Nche, G. C. (2013). European and American Christian Missions and Nigeria's National Development (1840-1960). Journal of Educational and Social Research, 3(10), 89-99.

Dickey, H., Watson, V., \& Zangelidis, A. (2011). Is it all about money? An examination of the motives behind moonlighting. Applied Economics, 43, 3767-3774.

Dieleman, M., Cuong, P., Anh, L., \& Martineau, T. (2003). Identifying factors for job motivation of rural health workers in North Vietnam. Human Reseources for Health, $1(10), 1-10$.

Dolgin, J. L., \& Dieterich, K. R. (2012). Social and legal debate about the Affordable Care Act. University of Missouri at Kansas City Law Review 80, 45-90.

Donabedian, Wheeler, J. R. C., \& Wyszewianski, L. (1982). Quality, cost, and health: An integrative model. Med. Care, 20, 975 -992.

Donabedian, A. (1980). The Definition of Quality and Approaches to its Assessment. Vol 1. Explorations in Quality Assessment and Monitoring Ann Arbour, MI, Health Administration Press, 1-164.

Donabedian, A. (1992). Quality assurance in healthcare: a consumers' role. Quality in HealthCare, 1, 247-251.

Donabedian, A. (1997). The quality of Care: How can it be assessed? Arch Pathol Lab Med, $121,1743-1748$.

Donabedian, A. (2005). Evaluating the Quality of Medical Care. The Milbank Quarterly Vol. 83(No. 4), (pp. 691-729).

Doyle, Y., \& Bull, A. (2000). Role of private sector in United Kingdom health care system. BMJ, 321, 563-565.

Eggleston, K., \& Bir, A. (2006). Physician dual practice. Health Policy, 78(2-3), 157-166. doi: 10.1016/j.healthpol.2005.09.007

Ensor, T., \& Weinzierl, S. (2007). Regulating health care in low- and middle -income countries: Broadening the policy response in resource contrained environments Social Sceinece and Medicine 65, 355-366.

Eze, B. S., Ochonma, O. G., Ajuba, M., \& Obikeze, E. (2015). Contextual access challenges to the antiretroviral therapy for HIV-infected persons in southeast Nigeria. American Journal of Research Communication, 3(11).

Fapohunda, BM, \& Orobaton, NG (2013). When Women Deliver with No One Present in Nigeria: Who, What, Where and So What? PLOS One, 8(7).

Farrell, C. (2004). Patient and Public Involvement in Health: The Evidence for Policy Implementation. London: Department of Health, 1-11.

Federal Ministry of Health. (2005). National Policy on Public Private Partnerships for Health in Nigeria. Abuja, Nigeria. Retrived from http://cheld.org/wpcontent/uploads/2012/04/National-Policy-on-Public-and-Private-Partnership-forHealth-in-Nigeria.pdf. 
Constitution of the Federal Republic of Nigeria (1999). Certain Consequential Repeals Decree No 63 of 1999 Laws of the Federation of Nigeria. Retrieved from http://www.nigerialaw.org/Constitution\%20of\%20the\%20Federal\%20Republic\%20of \%20Nigeria\%20(Certain\%20Consequential\%20Repeals)\%20Decree\%201999.htm

Ferrinho, P., Larberghe, W. V., Julie, M.R., Fresta, E., Gomes, A., Dias, F., Gonçalves, A., Backstrom, B. (1998). How and why public doctors engage in private practice in Portuguese-speaking African countries. Health Policy And Planning, 13(3), 332-338.

Ferrinho, P., Lerberghe, W. V., Fronteira, I., Hipolito, F., \& Biscaia, A. (2004b). Dual practice in the health sector: review of the evidence. Human Resource for Health, 2(14) doi: doi: 10.1186/1478-4491-2-14

Ferrinho, P., Omar, M. C., Fernandes, M., Blaise, P., Bugalho, A. M., \& Lerberghe, W. V. (2004a). Pilfering for survival : How health workers use access to drugs as a coping strategy. Human Resource for Health, 2(4).

Filmer, D., \& Pritchett, L. (2001). Estimating wealth effects without expenditure data - or tears: an application to educational enrollments in states of India. Demography 38, 115-132.

Flood, A. B., Scott, R. W., Ewy, W., \& Forrest, W. H. (1982). Effectiveness in Professional Organizations: The Impact of Surgeons and Surgical Staff Organization on the Quality of Care in Hospitals. Health Services Research, 17(4), 341-373.

Flood, C., \& Archibald, T. (2001). The illegality of private health care in Canada. CMAJ, 164(6), 825-830

FMOH. (2011). A Directory of Health Facilities in Nigeria. Federal Ministry of Health (pp. 269-289). Abuja , Nigeria.

France, G., \& Donatini, A. (2005). The Italian health-care system. Health Economics, 14, 187-202.

Freidson, E. (1970a). Profession of Medicine. Chicago: University of Chicago Press.

Freidson, E. (1970b). Professional Dominance Chicago Aldine Publishing Company.

Freidson, E. (1994). Professionalism reborn: Theory, prophecy and policy. Chicago University of Chicago Press.

Gabric, N., \& Lazic, R. (2013). Private sector medicine-Increasing excellence and viable alternative to state sector. Medicina Academica Mostariensia, 1(1), 41- 44.

Garcia-Prado, A., \& Gonzalez, P. (2007). Policy and regulatory responses to dual practice in the health sector. Health Policy, 84(2-3), 142-152. doi: 10.1016/j.healthpol.2007.03.006

Garcia-Prado, A., \& Gonzalez, P. (2011). Whom do physicians work for? An analysis of dual practice in the health sector. J Health Polit Policy Law, 36(2), 266-294. doi: $10.1215 / 03616878-1222721$

Giaimo, S. (1995). Health Care Reform in Britain and Germany: Recasting the Political Bargain with the Medical Profession. Governance, 8(3), 354-379.

Gilson, L., Alilio, M., \& Heggenhougen, K. (1994). Community satisfaction with primary health care services: an evaluation undertaken in the Morogoro region of Tanzania. Soc. Sci Med, 39, 767-780.

Giuffrida, A., Gravelle, H., \& Roland, M. (1999). Measuring quality with routine data: avoiding confusion between performance indicators and health outcomes. Br. Med. J., 319, 94- 98 . 
Gladstone, D. (2000). Regulation, Acccountability, and Healthcare, Pp. 1-7 in Gladstone , D. (ed) Regulating Doctors. London: Institute for the study of Civil society.

Godager, G., \& Luras, H. (2009). Dual job holding general practitioners: the effect of patient shortage. [Research Support, Non-U.S. Gov't]. Health Econ, 18(10), 133-145. doi: $10.1002 /$ hec. 1418

Gonzalez, P. (2004). Should physicians' dual practice be limited? An incentive approach. Health Economics, 13, 505-524.

González, P., \& Macho-Stadler, I. (2013). A theoretical approach to dual practice regulations in the health sector. Journal Of Health Economics, 32(1), 66-87. doi: 10.1016/j.jhealeco.2012.08.005

Green, J., \& Thorogood, N. (2014). Qualitative Methods for Health Research (Third ed.). London: SAGE.

Gruen, R., Anwar, R., Begum, T., Killingsworth, J. R., \& Normand, C. (2002). Dual job holding pratitioners in Bangladesh: An exploration. Social Science and Medicine, 54 267-279.

Gulliford, M., Figueroa-Munoz, J., Morgan, M., Hughes, D., Gibson, B., Beech, R, \& Hudson, M. (2002). What does 'access to health care' mean? Journal of Health Services Research \& Policy, 7(3), 186-188.

Hamilton, S. M., Letourneau, S., Pekeles, E., Voaklander, D., \& Johnston, D. W. C. (1997). The Impact of Regionalization on a Surgery Program in the Canadian Health Care System. Archives of Surgery, 132, 605-611.

Hammersley, M. (1989). The dilenma of Qualitative Method. London: Routledge.

Hanae, I. E. H., Mohamed, L., \& Noureddine, R. (2013). Quality of care between Donabedian model and ISO9001V2008. International Journal for Quality Research 17, 7(1), 17 30 .

Hanson, K., Gilson, L., Goodman, C., Mills, A., Smith, R., Feachem, R., . . . Kinlaw, H. (2008). Is Private Health Care the Answer to the Health Problems of the World's Poor? PLOS Medicine, 5(11), e233, 1528-1532 doi: 10.1371/journal.pmed.0050233

Hanson, K., \& Jack, W. (2010). Incentives Could Induce Ethiopian Doctors And Nurses To Work In Rural Settings. Health Affairs 29(8), 1452-1460. doi: 10.1377/hlthaff.2009.0164, 1452-1460.

Hanvoravongchai, P., Letiendumrong, J., Teerawattananon, Y., \& Tangcharoensathien, V. (2000). Implications for private practice in public hopsitals on the cesarean section rate in Thailand. Human Resources for Health Development Journal, 4, 1-2.

Harrison, S., Hunter, D. J., \& Pollitt, C. (1990). The Dynamics of British Health Policy. London: Unwin.

Harrison, S., \& Pollitt, C. (1994). Controlling Health Professionals: The Future of Work and Organisation in the NHS (Vol. 80). Buckingham: Open University Press.

Health Careers, (2017). Pay for doctors. Retrieved from https://www.healthcareers.nhs.uk

Heather, L., \& Richard, H. G. (2011). Do hospitalist physicians improve the quality of inpatient care delivery? A systematic review of process, efficiency and outcome measures. BMC Medicine, 9(58), 2-22. 
Heikkilä, T. J., Hyppölä, H., Aine, T., Halila, H., Vänskä, J., Kujala, S., . . . Mattila, K. (2014). How do doctors choose where they want to work? Motives for choice of current workplace among physicians registered in Finland 1977-2006. Health Policy, 114(2), 109-117. doi: 10.1016/j.healthpol.2013.12.001

Helen, R. M., Joanna, L., Willetts, M. S., Tin-Chi, L., Melanye, J. B., \& Santosh, K. V. (2014). Work in Multiple Jobs and the Risk of Injury in the US Working Population. American Journal of Public Health 104(1), 134-142.

Henderson, L., \& Tulloch, J. (2008). Incentives for retaining and motivating health workers in Pacific and Asian countries. Human Resorces for Health 6(18), 1-20.

Hipgrave, D. B., \& Hort, K. (2013). Dual practice by doctors working in South and East Asia: A review of its origins, scope and impact, and the options for regulation. Health Policy And Planning, 54(2), 267-279.

Hipple, S. F. (2010). Multiple jobholding during the 2000s. Monthly Labour Review, 21-32.

Hoa, N. Q., Ohman, A., Lundborg, C. S., Thi Kim Chuc, N. (2007). Drug use and healthseeking behaviour and challenges for childhood illnes in Vietnam - a qualitatve study. Health Policy 82, 320-329.

Hongoro, C., \& Kumaranayake, L. (2000). Do they work? Regulating for-profit providers in Zimbabwe. Health Policy and Planning 15(4), 368-377.

Horton, R., \& Clark, S. (2016). The perils and possibilities of the private health sector. The Lancent 388.

Hughes, D. (2014) Health administration, In Cockerham, W.C., Dingwall, R. and Quay, S.(eds) The Wiley-Blackwell Encyclopedia of Health, Illness, Behavior and Society. Oxford: Wiley-Blackwell.

Hughes, D. (1993). General practitioners and the new contract: promoting better health through financial incentives. Health Policy, 25, 39-50.

Hughes, D. R., Behargavan, M., \& Sunshine, J. H. (2010). Imaging self-referral associated with higher costs and limited impact on duration of illness. Health Affairs, 29(12), 2244-2251.

Humphrey, C., \& Russell, J. (2004). Motivation and values of hospital consultants in southeast England who work in the national health service and do private practice. Social Science \& Medicine 59(6), 1241-1250.

Ichoku, H., Fonta, W. M., \& Ataguba, J. E. (2013). Political economy and history: making sense of health financing in sub-Saharan Africa. Journal of International Development, 25, 297-309.

Institute of Medicine. (2001). Crossing the quality chasm: A new health system for the 21st century. Washington, DC: National Academy Press

Isaac, T., Zazlavsky, A. M., Cleary, P. D., \& Landon, B. E. (2010). The relationship between patients' perception of care and measures of hospital quality and safety. Health Services Rserach. 45(4):1024-40.

Isah, E. C., Omorogbe, V. E., Orji, O., \& Oyovwe, L. (2008). Self-reported absenteeism among Hosipital workers in Benin City Nigeria. Ghana Medical Journal 42(2), 2-7.

Iyi, E. A. (2014). A Review of Enugu (Enugu State, Nigeria) Urban Growth and Development. Journal of Research in Environmental and Earth Sciences, 1(3), 44-51.

Izugbara, C. O., \& Wekesah, F. (2017). What does quality maternity care mean in a context of medical pluralism? Perspectives of women in Nigeria. Health Policy and Planning, $33,1-8$. 
Jacobs, J. (1988). Doctors and Rules: A Sociology of Professional Values. London: Routledge.

Jamshidi, R., \& Reilly, L. (2008). Surgical Residents' Clinical Activity During Research: Shedding Light on Moonlighting practices. Journal of Surgical Education, 65(6), 48693

Jan, S., Bian, Y., Jumpa, M., Meng, Q., Nyazema, N., Prakongsai, P., \& Mills, A. (2005). Dual job holding by public sector health professionals in highly resource-constrained settings: problem or solution? [Research Support, Non-U.S. Gov't]. Bull World Health Organ, 83(10), 771-776. doi: /S0042-96862005001000014

Johannessen, K. A., \& Hagen, T. P. (2014). Physician engagement in dual practices and the effects on labor supply in public hospitals: results from a register-based study. [journal article]. BMC Health Services Research, 14:299. doi: 10.1186/1472-6963-14-299, 111.

Joseph, J. G., Emmons, C., Kessler, R. C., Wortmann, C. B., O'Brien, K., Hocker, W. T., \& Shaefer, C. (1984). Coping with the threat of AIDS: An approach to psychosocial assessment . American Psychologist, 39(11), 1297-1302.

Jumpa, M., Jan, S., \& Mills, A. (2007). The role of regulation in influencing incomegenerating activities among public sector doctors in Peru. Human Resources For Health, 5:5.

Kamorudeen, A., \& Abdulkareem, S. B. (2012). Corruption in the Nigerian public health care delivery system. Sokoto Journal of the Social Sciences 2 (2), 98-114.

Kanji, N., Kilima, P., Lorenz, N., \& Garner, P. (1995). Quality of primary outpatient services in Dar-es Salaam: a comparison of govenment and voluntary providers . Health Policy And planning, 10 (2).

Kankaanranta, T., Nummi, T., Vainiomaki, J., Halila, H., Hyppola, H., Isokoski, M., Kujala S, Kumpusalo E, Mattila K, Virjo I, Vänskä J, \& Rissanen, P. (2007). The role of job satisfaction, job dissatisfaction and demographic factors on physicians' intention to switch work sector from public to private. Health Policy, 83(1), 50-64.

Kaufmann, D., Kraay, A., \& Mastruzzi, M. (2006). Governance matters V: Aggregate and individual governance indicators for 1996-2005. Retrieved from http://siteresources.worldbank.org/DEC/Resources/GM5.pdf.

Kiwanuka, SN. Kinengyere, AA. Nalwadda, C., Ssengooba, F., Okui, O., Pariyo, GW. Effects of interventions to manage dual practice. Cochrane Database of Systematic Reviews 2010, Issue 3. Art. No.: CD008405. DOI: 10.1002/14651858.CD008405.

Kiwanuka, S. N., Rutebemberwa, E., Nalwadda, C., Okui, O., Ssengooba, F., Kinengyere, A. A., \& Pariyo, G. W. (2011). Interventions to manage dual practice among health workers. [Review]. Cochrane Database Syst Rev(7), CD008405. doi: 10.1002/14651858.CD008405.pub2

Klein, R. (2006). The New Politics of the National Health Service: From Creation to Reinvention (5th edn ). Oxford: Radcliffe.

Kohn, L., Corrigan, J., \& Donaldson, M. (eds.) (1999). To Err is Human: Building a Safer Health System. Washington, DC: National Academy Press.

Kouri, B. E., Parsons, R. G., \& Alpert, H. R. (2002). Physician self-referral for diagnostic imaging: Review of the empiric literature. AJR, 179, 843-850. 
Kruk, M., E., \& Freedman, L. P. (2008). Assessing health system performance in developing countries: A review of the literature. Health Policy, 85, 263-276.

Kumaranayake, L. (1997). The role of regulation: Influencing private sector activity within health sector reform. Journal of International Development 9(4), 641-649.

Kumaranayake, L., Lake, S., Mujinja, P., Hongoro, C., \& Mpembeni, R. (2000). How do countries regulate the health sector? Evidence from Tanzania and Zimbabwe. Health Policy and Planning, 15(4), 357-367.

LaGrone, L., Isquith-Dicker, L., Egoavil, E., Herrera-Matta, J., Fuhs, A., Checa, D., Revoredo, F., Rodriguez Castro, M.J.A. Mock, C. (2017). Surgery and trauma care providers' perception of the impact of dual-practice employment on quality of care provided in an Andean country. BJS 104 (6), 704-709. doi:10.1002/bjs.10478.

Larry, R. H., Jeffrey, A., Alexander, I. F., \& Joanna, J. H. (2008). How do hospital organizational structure and processes affect quality of care? : A critical review of research methods. Med Care Res Rev 65 (3), 259-299.

LaVela, S., \& Gallan, A. (2014). Evaluation and measurement of patient experience. Patient Experience Journal, 1(1), 28-36.

Lavrakas, P. (ed.) (2008). Encyclopedia of Survey Research Methods. Retrived from

http://methods.sagepub.com/reference/encyclopedia-of-survey-research-methods

Lerberghe, W., Conceic, ã o, C., Damme, W., \& Ferrinho, P. (2002). When staff is underpaid: dealing with the individual coping strategies of health personnel. Bulletin of the World Health Organization 80(7), 581-584.

Li, J., Tabor, R., \& Martinez, M.. (2000). Survey of Moonlighting Practices and Work Requirements of Emergency Medicine Residents. American Journal, of Emergency Medicine, 18(2), 147-151.

Light, D. W. (1991). Professionalism as a Countervailing Power. Journal of Health Politics, Policy \& Law 16(3), 499-506.

Ligia, P. B. (2014). Dual practice in Kampala, Uganda: A mixed methods study of management and policy (Unpublished PhD Thesis). Johns Hopkins University, USA.

Lindelow, M., \& Serneels, P. (2006). The performance of health workers in Ethopia : results from qualitatve research. Soc Sci Med, 62(2006), 2225-2235.

Lisa, G. K, Eddie, K., Arur, A., \& Sanjana, P. (2009). Nigeria Health System Assessment 2008. Abt Associates Inc. Health Systems 20/20 (pp. 135).

Loh, L. C., Ugarte-Gilb, C., \& Darko, K. (2013). Private sector contributions and their effect on physician emigration in the developing world. Bull World Health Organ, 91, 227233. doi: 10.2471/BLT.12.110791

Low, J.(2013). Unstructured and semi-structured interviews in Health Research . Pp. 88-102 in Saks, M \& Allsop, J. (eds.). Researching Health: Qualitative , Quantitative and Mixed methods ( $2^{\text {nd }}$ ed) London: SAGE.

Macq, J., Ferrinho, P., Brouwere, V. D., \& Lerberghe, W. V. (2001). Managing Health Services in Developing Countries: Between the Ethics of the Civil Servant and the Need for Moonlighting: Managing and Moonlighting. Human Resources for Health Development Journal (HRDJ) 5 (1-3), 17-24.

Mariynn, M. R. (1992). Growth of private medicine in Sweden:The new diversity and the new challenge. Health Policy, 21(2), 155-166. 
Mattke, S., Epstein, A. M., \& Leatherman, S. (2006). The OECD Health Care Quality Indicators project: history and background. International Journal for Quality in Health Care, 18 Suppl 1: 1-4.

Maxwell, J. A., \& Mittapalli, K. (2010). SAGE Handbook of Mixed Methods in Social \& Behavioral Research ( $2^{\text {nd }}$ ed.). Thousand Oaks, California: SAGE Publications, Inc. Retrieved from http://methods.sagepub.com/book/sage-handbook-of-mixed-methodssocial-behavioral-research-2e.doi: 10.4135/9781506335193

Maxwell, R. J. ( 1984). Quality assessment in health. Br. Med. J, 288, 1470-1472.

Mays, N., \& Pope, C. (2000). Assessing quality in qualitative research. BMJ 2000, 320, 5052.

McCoy, D., Bennett, S., Witter, S., Pond, B., Baker, B., Gow, J., Chand, S., Ensor, T. \& McPake, B. (2008). Salaries and incomes of health workers in sub-Saharan Africa. The Lancet, 371(9613), 675-681. doi:https://doi.org/10.1016/S01406736(08)60306-2

McKenzie, A., Sokpo, E., \& Ager, A. (2014). Bridging the policy-implementation gap in federal health systems: lessons from the Nigerian experience. Journal of Public Health in Africa, 5(381), 82-87.

McPake, B., Russo, G., \& Tseng, F.M. (2014). How do dual practitioners divide their time? The cases of three African capital cities. Social Science \& Medicine, 122, 113-121. https://doi.org/10.1016/i.socscimed.2014.10.040

MDCN. (2008). The Code of Medical Ethics in Nigeria. Abuja: Medical and Dental Council of Nigeria.

Mills, A., Brugha, R., Hanson, K., \& McPake, B. (2002). What can be done about the private health sector in low-income countries? Bulletin of the World Health organization, 80(4), 325-330.

Mitchell, J. M., \& Scott, E. (1992). Physician ownership of physical therapy services. Effects on charges, utilization, profit, and service characteristics. The Journal of the American Medical Association, 268(15), 2055-9.

Mitchell, J. M. (2005). Effects of physician-owned limited-service hospitals: evidence from Arizona. Health affairs (Project Hope), Suppl Web Exclusives, W5-481-490. doi: 10.1377/hlthaff.w5.481

Mitchell, J. M., \& Sass, T., M. (1995). Physician ownership of ancillary services: Indirect demand inducement or quality assurance. Journal of Health Economics, 14, 263-289.

Moghri, J., Arab, M., Rashidian, A., \& Sari, A. A. (2016). Physician Dual Practice: A Descriptive Mapping Review of Literature. Iran J Public Health, 45(3), 278-288.

Mohammed, S., Souares, A., Bermejo, J. L., Sauerborn, R., \& Dong, H. (2014). Performance evaluation of a health insurance in Nigeria using optimal resource use: health care providers perspectives. BMC Health Services Rsearch 14(127).

Morakinyo, O., \& Fagbamigbe, A. (2017). Neonatal, infant and under-five mortalities in Nigeria: An examination of trends and drivers (2003-2013). PLOS ONE, 12(8), 1-21. https://doi.org/10.1371/journal.pone.0182990.

Morgan, D. L. (1997). Focus Groups as Qualitative Research (2nd ed. Vol. 16). London: SAGE.

Morris, S., Bob, E., Ma, A., McConnachie, A., Rice, N., Skatun, D., \& Sutton, M. (2008). Analysis of consulatants' NHS and private incomes in England in 2003/4. J R Soc Med, 101, 372-380. doi: 10.1258/jrsm.2008.080004 
Mossialos, E., Allin, S., \& Davaki, K. (2005). Analysing Greek health system: A tale of fragmentation and inertia. Health Economics, 14, S151- S168.

Murphy, E., Dingwall, R., Greatbatch, D., Parker, S., \& Watson, P. (1998). Qualitative research methods in health technology assessment: a review of the literature. Health Technology Assessment 2(16)

Musa, B. M., \& Musa, A. G. (2014). The burden of non-communicable diseases in Nigeria in the context of globalization. Ann Afr med 13, 1-10.

Muula, A. S., \& Maseko, F. G. (2006). How are health professionals earning their living in Malawi. BMC Health Services Research, 6(97).

National Population Commission, (2006). National Census, Abuja, Nigeria.

National Population Commission (2013). Nigeria Demographic and Health Survey Primary Report. Abuja, Nigeria.

NHS Foundation Trust. (2013). The Mid Staffordshire NHS Foundation Trust Public Inquiry (ISBN: 9780102981476). London.

Nitararumphong, S., Mills, A., Pongsupap, Y., \& Tancharoensatien. (2005). What is talked about less in Health Care Reform? Thailand: National Security Office.

Numerato, D., Salvatore, D., \& Fattore, G. (2012). The impact of management on medical professionalism: a review. Sociology of Health \& Illness, 34(4), pp. 626-644.

Obikeze, E., Onwujekwe, E., Uzochukwu, B., Chukwuogo, O., Uchegbu, E., Eze, S., \& Uguru, N. (2013). African Journal of Health Economics, 0002, 16.

Odigwe, C. (2012). Lagos state doctors are sacked for striking over pay. BMJ (344) doi: doi: 10.1136/bmj.e3512

Ogunbekun, I., Ogunbekun, A., \& Orobaton, N. (1999). private health care in Nigeria: walking the tightrope. Health Policy and Planning, 14(2), 174-181.

Ogundeji, Y. K., Jackson, C., Sheldon, T., Olubajo, O., \& Ihebuzor, N. (2016). Pay for performance in Nigeria: The influence of context and implementation on results. [Article]. Health Policy and Planning, 31(8), 955-963. doi: 10.1093/heapol/czw016

Okoronkwo, I. L., Onwujekwe, E. O., \& Ani, F. O. (2014). The long walk to universal health coverage: patterns of inequities in the use of primary healthcare services in Enugu, Southeast Nigeria. BMC Health Services Research 14(132) doi: 10.1186/1472-6963$14-132$

Olejaz, M., Juul , N., Rudkjøbing, A., Okkels, B., Krasnik, A., \& Hernández-Quevedo, C. (2012). Denmark: Health System Review. Health Systems in Transition. Retrieved from

http://www.euro.who.int/_data/assets/pdf_file/0004/160519/e96442.pdf?ua=1

Oliveira, M., \& Pinto, C. (2005). Health care reform in Portugal: an evaluation of the NHS experience. Health Economics, 14, S203-S220.

Ojo, T., \& Akinwumi, A. (2015). Doctors as managers of healthcare resources in Nigeria: Evolving roles and current challenges. Journal of the Nigeria Medical Association 56(6), 375-380. doi: http://doi.org/10.4103/0300-1652.171614

Olowookere, A. A., Fatiregun, A. A., Ladipo, M. M., \& Akenova, Y. A. (2011). Reducing waiting time at a Nigerian HIV Treatment Clininc: Opinions from and the satisfaction of People Living with HIV/AIDS. Journal of the International Association of Physicians in AIDS Care (JIAPAC), 11(3), 188-191. 
Onah, M. N., \& Govender, V. (2014). Out-of-pocket payment, health care access and utilization in south-eastern Nigeria: a gender perspective. PLOS ONE, 9(4), 1-11. doi: doi:10.1371/journal.pone.0093887

Onwujekwe, O., Hanson, K., \& Uzochukwu, B. (2012). Examining Inequities in Incidence of Catastrophic Health Expenditures on Different Healthcare Services and Health Facilities in Nigeria. PLoS ONE, 7(7), 1-6. doi: e40811. doi:10.1371/journal.pone.0040811

Onwujekwe, O., Hanson, K., Uzochukwu, B., Ezeoke, O., Eze, S., \& Dike, N. (2010). Geographic inequities in provision and utilization of malaria treatment services in southeast Nigeria: Diagnosis, providers and drugs. Health Policy, 94, 144-149.

Onwujekwe, O. E., Ojukwu, J., Ezumah, N., Uzochukwu, B., Dike, N., \& Eze, S. (2006). Socio-economic differences in preferences and willingness to pay for different providers of malaria treatment in southeast Nigeria. Am. J. Trop. Med. Hyg.,, 75(3), 421-429.

Onyeji, E. (2017, Nov. 10). 2018 Budget: Again, Nigeria falls short of AU health funding commitment. Retrived from

https://www.premiumtimesng.com/news/headlines/248909-2018-budget-nigeria-fallsshort-au-health-funding-commitment.html.

Orubuloye, I. U., \& Oni, J. B. (1996). Health transition research in Nigeria in the era of the Structural Adjustment Programme. Health Transition Review, Supplement 6, 301-324.

Osuagu, E. M. (2010). Ethics and Medico legal Aspects of Medical Practice. Enugu, Nigeria: Jaron Industries Ltd.

Ovretveit, J. (1985). Medical dominance and the development of professional autonomy in physiotherapy. Socioiogy of Health and Illness 7 (1), 77-93.

Palmer, N., Mills, A., Wadee, H., Gilson, L., \& Schneider, H. (2003). A new face for private providers in developing countries: what implications for public health? Bulletin of the World Health Organization 81( 4), 292-296.

Parasuraman, A., Zeithaml, V., \& Berry, L. (1985). A conceptual model of service quality and its implication for future research. Journal of Marketing 49(1), 41-50.

Perryman, E. (2017). UK private healthcare market to grow by $2.8 \%$ a year to 2025 Retrieved from https://www.healthinsurancedaily.com/healthinsurance/product-area/pmi/article482713.ece

Peters, T. (1987). Thriving on Chaos: Handbook for a Management Revolution. . New York: Knopf. Inc.

Pietro, C. D. (2006). Private medical services in the Italian public hospitals: The case for improving HRM. Health Policy 78(1), 56-69.

Pongsupap, Y., \& Van Lerberghe, W. (2006). Choosing between public and private or between hopsital and primary care: responsiveness, patient-centredness and prescribing patterns in outpatint consultations in Bangkok. Trop Med Int Health, 11, 81-89.

Pope, C., \& Mays, N. (1995). Reaching the parts other methods cannot reach: an introduction to qualitative methods in health and health services research. BMJ:British Medical Journal, 311(6996), 42-45.

Powell, M., \& Miller, R. (2014). Framing privatisation in the English National Health Service. Journal of Social Policy, 43(03), pp 575 - 594. 
Qadeer, I., \& Reddy, S. (2013). Medical tourism in India: Perceptions of physicians in tertiary care hospitals. Philosophy, Ethics and Humanities in Medicine, 8(20), 1-10.

Rajkotia, Y., Zang, O., Nguimkeu, P., Gergen, J., Djurovic , I., Vaz, P., Mbofana, F. \& Jobarteh, K. (2017). The effect of a performance-based financing program on HIV and maternal/child health services in Mozambique - an impact evaluation. Health Policy and Planning, 32(10), 1386-1396.

Raleigh, V. S., \& Catherine, F. (2010). Getting the measure of quality, Opportunities and challenges. ISBN: 978185717590 5, The King's Fund: UK.

Ramsaran-Fowdar, R. (2005). Identifying health care quality attributes. J Health Hum Serv Adm., 27(4), 28-43.

Ran, L., Luo, K., Wu, Y., Yao, L., \& Feng, Y. (2013). An analysis of China's physician salary payment system. J Huazhong Univ Sci Technol [Med Sci], 33(2), 309-314.

Rao, J.N.K., and Scott, A.J. (1981). The Analysis of Categorical Data from Complex Sample Surveys: Chi-Squared Tests for Goodness of Fit and Independence in Two-Way Tables. Journal of the American Statistical Association. 76(374), pp. 221-230.

Rickman, N., \& McGuire, A. (1999). Regulating providers' reimbursement in a mixed market for health Care. Scottish Journal of Political Economy, 46(1), 53-71.

Robert, H. B., Elizabeth, A., McGlynn, \& Paul, G. S. (2000). Defining and measuring quality of care: a perspective from US researches. International Jounal of Quality in Healh Care, 12(4), 281-295.

Roenen, C., Ferrinho, P., Dormael, M. V., \& Conceicao, M. C. (1997). How African doctors make ends meet: an exploration. Tropical Medicine and International Health, 2(2), 127-135.

Rogers, A., Hassell, K., \& Nicholaas, G. ( 1999). Demanding Patients? Analysing the Use of Primary Care. Oxford: Oxford University Press.

Rokx, C., Giles, J., Satriawan, E., Marzoeki, P., Harimurti, P., \& Yavuz, E. (2010). New Insights into the Provision of Health Services in Indonesia: A Health Services in Indonesia: A Health Workforce Study. Directions in Development ; Human Development. Washington, DC: The World Bank.

Royce, R. (2009). A Study of the Dynamics of the Private Health Care Market in the United Kingdom with Particular Reference to the Impact of British United Provident Association (BUPA) Provider and Benefit Initiatives (Unpublished Ph.D. Thesis ). Swansea University, UK.

Russel, S. (2005). Treatment-seeking behaviour in uban Sri-lanka: trusting the state, trusting the private providers. Soc Sci Med, 61, 1396-1407.

Russo, G., McPake, B., Fronteira, I., \& Ferrinho, P. (2013). Negotiating markets for health: an exploration of physicians' engagement in dual practice in three African capital cities. Health Policy and Planning, 29, 774-783. doi: 10.1093/heapol/czt071

Sæther, M. E. (2003). A Discrete Choice Analysis of Norwegian Physicians' Labour Supply and Sector Choice Health Economics Research Programme at the University of Oslo. Working Paper 2003: 19 Oslo.

Saldana, J. (2013). The Coding Manual for Qualitative Researchers (Second ed.). London: SAGE.

Sale, D. (2005). Understanding Clinical Governance and Quality Assurance: Making It Happen. New York: Palgrave Macmillan. 
Salter, B. (2000). Change in the governance of medicine: the politics of self-regulation. Pp. 8-27 in Gladstone, D. (ed) Regulating Doctors. London: Institute for the Study of Civil Society.

Sankore, R. (2010). A Call for a Massive Paradigm Shift from Just Health Financing to Integrated Health, Population and Social Development Investment in Africa (The Case for Progressing from Only 15\% to 15\%+) presented at the AU Continental Conference on Maternal, Infant and Child Health in Africa, And the Maputo Plan of Action (MPoA) on Sexual and Reproductive Health and Rights (SRHR). 19-21 April.

Schuftan, C., \& Unger, J. P. (2011). The Rockefeller Foundation's "Public Stewardship of Private Providers in Mixed Health Systems": A point-by-point critique. Social Medicine 6(2), 128-136.

Scott-Emuakpor, A. (2010). The evolution of health care system in Nigeria: Which way forward in the twenty- first century. Niger. Med. J., 51(2)

SHOP Project, (2016). Nigeria private Health Sector Project: Final Report 2009-2016. Bethesda, MD: SHOPS Project, Abt Associates Inc.

Siddiqi, S., Hamid, S., Rafique, G. A., Chaudhry, S. A., Ali, N., Shahab, S., Sauerborn, R. (2002). Prescription practices of public and private health care providers in Attock District of Parkistan. Int Health Plann Manage, 17, 23-40.

Siddiqi, S., Tayyeb, I. M., Sania, N., P., D. H., Belgacem, S., Khalif, M. B., \& Mohamed, A. J. (2009). Framework for assessing governance of the health system in developing countries: Gateway to good governance. Health Policy 90, 13-25.

Silverman, D. (2011). Interpreting Qualitative Data (4th ed.). London: Sage.

Sima, B., Heywood, P., Sandy, O., \& Garner, P. (2011). Quality of private and public ambulatory health care in low-and-middle income countries: systematic reveiw of comparative studies. PLoS Medicine, 8(4)

Smith Conway, K \& Kimmel, J.. (1998). Male labor supply estimates and the decision to moonlight. Labour Economics 5(1998), 135-166.

Socha, K., \& Bech, M. (2011). Physician dual practice: A review of literature. Health Policy, 102(1). doi: 10.1016/j.healthpol.2010.10.017

Socha, K., \& Bech, M. (2012). Dual practitioners are as engaged in their primary job as their senior colleagues. Danish Medical Journal, 59(2), A4375-A4375.

Soeung, S. C., Grundy, J., Morn, C., \& Samnang, C. (2008). Evaluation of immunization knowledge, practice, and service -delivery in the private sector in Cambodia. $J$ Health Popul Nutr, 26, 95-104.

Special Military Tribunal Act, (1984). Regulatd and Other Professions (Private Practice Prohibition Act). CAP, 390 P. 13915-13920.

Stacey, M. (2000). The General Medical Council and professional self-regulation. Pp. 28-39 in Gladstone , D. (ed) Regulating Doctors. London: Institute for the Study of Civil Society.

Starr, P. (1982). The Social Transformation of American Medicine. New York: Basic Books.

Stata (2013). Survey Data Reference Manual Release 13. College Station, Texas: Stata Press.

Steven, D., \& Bazzoli, G. (1985). The moonlighting decisions of resident physicians. Journal of Health Economics, 4(283-292)

Strong, P. (1979). The Ceremonial Order of Clinic. Parents, Doctors and Medical Bureaucracies. London: Routledge and Kegan Paul. 
Swan, B., \& Boruch, R. (2004). Quality of evidence: usefulness in measuring the quality of health care Medical Care 42(2 Suppl II12-20)

Swedlow, A., Gregory, J., Smithline, N., \& Milstein. (1992). Increased costs and rates of use in the Califonia workers' compensation system as a result of self-referral by physicians. The New England Journal of Medicine 327(21), 1502-1506.

Thorne, T. (2016). How could the Quality and Outcomes Framework (QOF) do more to tackle health inequalities? London Journal of Primary Care, 8(5), 80-84. doi: 10.1080/17571472.2016.1215370

Umar, I., Ochem, O., \& Umar, A. S. (2011). Patient waiting time in a tertiary health institution in Northern Nigeria. Journal of Public Health and Epidemiology, 3 (2), 78 82.

UNDP (2016). Human Development Report: Human Development for Everyone. Retrieved from http://hdr.undp.org/sites/default/files/2016_human_development_report.pdf

Uzochukwu, B., Onwujekwe, O., \& Ezumah, N. (2014). The district health system in Enugu State, Nigeria: An analysis of policy development and implementation. African Journal of Health Economics 3(1), 14.

Vries, H., Sanderson, P., Janta, B., Rabinovich, L., Archontakis, F., Sharif, I., Klautzer, L., Marjanovic, S., Patruni, B., Puri, S. \& Tiessen, J. (2009). International Comparism of Ten Medical Regulatory Systems. Cambridge, United Kingdom. Technical Report. Rand Europe., PP.83-91.

Vujicic, M., Shengelia, B., Alfano, M., \& Thu, H. B. (2011). Physician shortages in rural Vietnam: using a labor market approach to inform policy. Social Science \& Medicine 73(7), 970-977. doi: 10.1016/j.socscimed.2011.06.010

Vyas, S., \& Kumaranayake, L. (2006). Constructing socio-economic status indices: how to use principal components analysis. Health Policy and Planning. 21(6), 459-68. doi: 10.1093/heapol/czl029

Walker, S., Mason, A., Claxton, K., Cookson, R., Fenwick, E., Fleetcroft, R., \& Sculpher, M. (2010). Value for money and the Quality and Outcomes Framework in primary care in the UK NHS. British Journal of General Practice. 60(574), e213-20. doi: 10.3399/bjgp10X501859

World Data Atlas, (2012). Health expenditure as a share of GDP in Nigeria. Retrieved from (https://knoema.com/atlas/Nigeria/Health-expenditure-as-a-share-of-GDP

WHO, (2000). The World Health Report Health Systems: Improving Performance. Geneva, Switzerland.

WHO, (2007). Everybody's business: strengthening health systems to improve health outcomes. WHO's Framework for Action (pp. 2-7). Geneva, Switzerland.

WHO, (2016). WHO Key Country Indicators:Nigeria key Indicators. Retrieved from http://apps.who.int/gho/data/node.cco.ki-NGA?lang=en,

Wiley, M. (2005). The Irish health system: developments in strategy, structure, funding and delivery since 1980. Health Economics 14(S1), 169-186. https://doi.org/10.1002/hec.1034.

Wilson, I. B., Landon, B.E., V., Marsden, P.V., Hirschkorn, K., McInnes, K., Ding, L., \& Cleary, P. D. (2007). Correlations among measures of quality in HIV Care in the United states: Cross sectional study. British Medical Journal.335(7629), 1085.

Winter, A. (2003). Comparing The Mix Of Patients In Various Outpatient Surgery Settings. HEALTH AFFAIRS. Vol.22 (6), 68-75 
https://doi.org/10.1377/hlthaff.22.6.68.

Yang, J. (2006). The privatisation of professional knowledge in the public health care sector in China. Health Sociology Review 15(1), 16-28.

Yates, J. (1987). Why Are We Still Waiting? An Analysis of Hospital Waiting Lists Oxford: Oxford University Press.

Yates, J. (2000). Consulatants' Contracts, Politicians' Dilemma and Patients' Inequity : Select Committe on Health Minutes of Evidence. Retrieved from https://publications.parliament.uk/pa/cm199900/cmselect/cmhealth/586/0061504.htm, 2015.

Yawson, A., Biritwum, R., \& Nimo, P. (2012). Effects of consumer and provider moral hazard at a municipal hospital out-patient department on Ghana's national health insurance scheme. Ghana Medical Journal 46(4), 200-210.

Zhang, X. (2015). Understanding the Current Situation and Challenges in the Public Private Mix (PPM) of Human Resources for Health (HRH) in Selected Areas in Egypt (Unpublished Master of Science Degree). Duke University, USA. 


\section{APPENDICES}

\section{Appendix 1: Focus group discussion guide}

\section{Introduction}

Good morning/afternoon.

I'm (Name of PI) from Swansea University, United Kingdom. I am in Nigeria to carry out a research on dual practice of medical professionals in southeast, Nigeria. The aim of this research is to carry out an economic and policy analysis of dual practice of medical professionals in public health facilities in southeast Nigeria. This is for academic purposes only.

The reason why we have called for this meeting is to discuss your perspectives, as important stakeholders in health care, what your views on dual practice are, how DP enhances or constrains health care services you receive in public health facilities. In doing this, we shall discuss your experiences in receiving health care services from the public sector and private practice managed by dual practitioners.

Before, we start I just want to make a final check that everybody is happy to go ahead, and that we do have your consent to participate.

If you are all happy we will go around the table and get people to introduce themselves.

\section{Major question:}

\section{Perceptions of dual practice of government doctors}

1. What are your perceptions of dual practice of medical professionals in Nigeria? (The investigator explains the term 'dual practice' to participants)

\section{Benefits and disbenefits of dual practice}

2. What are the benefits of dual practice? - to service users? (access to doctor any time, quicker services, flexibility of time of visit, deferred payment, benefits to government hospitals?

-Probe for dis-benefits: to service users? - Probe for unavailability of doctors in the public service when needed, probe for self-referral, cost of services in private practice, probe for time spent with doctor in government hospital, probe for disbenefits to public system?

\section{Hospital visit experiences}


3. How would you compare your experiences in public hospitals and private practices managed by dual practitioners? - probe for ease of access to services, cost/affordability of services, proximity, child delivery/ maternity services, quality of service, availability of doctors /time of availability, responsive to your health care needs etc.

4. How would you compare costs of services between government hospitals and private practices managed by dual practitioners? For instance, in malaria treatment, antenatal services, childhood treatment, child delivery, outpatient, inpatient services, any other areas of interest?

Probe for fee exemptions, reduced cost, deferred payments,

\section{Self-referral}

5. What are your perceptions of self-referral by public doctors who own private clinics? (Investigator explains the term self-referral)

- probe whether they have been self-referred from public to private practice

- probe for how respondents were self-referred (pattern used, use of business cards, using a third party, etc.)

6. Does self-referral affect services you suppose to obtain in the public facility? How? - probe for cost increase, lack of interest in public facility, accessing public facility only when the disease is too severe,

\section{Quality of care}

7. How do you assess quality of health care?

8. What characteristics do you use to assess quality of care and why?

- Probe for structure element of care, process and outcome

(Physical infrastructure and equipment- number of human resources, availability of staff, etc.- Probe for process (description of what was actually done - interpersonal) e.g. waittime, courtesy of provider, confidentiality, privacy, history taken, etc. - Probe for outcome element (effect of care on health status of patient), recovery, re-admission, morbidity, improvement in patient knowledge about the disease treated for - Probe for experiences where they had high quality care and poor quality care 9) How would you compare quality of healthcare in the public and in the private practice managed by dual practitioner 10). How do you differentiate good quality service from bad quality service? 11. Overall, how would you assess your care satisfaction between public facility and private practice managed by dual practitioner.

Conclusion.

Thank you 


\section{College of Health and Human Sciences}

Swansea University, United Kingdom

Project Title: Dual practice of medical professionals in public hospitals in Nigeria: An economic and policy analysis

Introduction: My name is Bartholomew $\mathrm{S}$. Eze, a $\mathrm{PhD}$ research student at Swansea University, United Kingdom. I am in Nigeria to carry out a study on the above project title. I would like to formally invite you to take part in this study. But before you decide, please take time to read the information below carefully. Please feel free to ask questions if anything is unclear or if you would like further information. Take time to decide whether or not you wish to take part.

Thank you for taking time to read it.

Purpose of the project: This study seeks to investigate dual practice of medical professionals who engage in public and private sector work simultaneously. Dual practice is a situation where public sector doctors also engage in private practice. The study will explore the benefits and disbenefits of this practice to service users. It will also examine service users' perceptions of this practice. Furthermore, it will gather opinions from policy makers, medical professionals and other stakeholders about current regulatory mechanisms for dual practice in Nigeria and options for policy reform.

Why have I been selected/do I have to take part? You have been selected based on your suitability for this study. It is up to you to decide whether or not to take part. You will not suffer any disadvantage if you decide not to participate. If you do decide to take part, you will be given this information sheet to keep and will be asked to sign a consent form. It is important to note that if you decide to take part, you are still free to withdraw at any time without giving a reason.

What will happen to me if I take part? You will be asked to complete a questionnaire. The completion of a questionnaire will take approximately 20 minutes.

Will my participation be kept confidential? We will not include your name or any other information that might identify you in the questionnaire. The questionnaire will be destroyed after the contents have been analysed and the findings written up.

What are the potential risks to you in taking part? There is no anticipated potential risk involved in this study. However, you will be required to take some time off your busy schedule to answer our questions. What happens next? If you are happy to be involved in 
this project, you will be asked to sign a consent form to confirm willingness. Researcher contact details: Bartholomew S. Eze, College of Health and Human Sciences, Swansea University, Singleton Park, Swansea, SA2 8PP, UK. sbeze09@gmail.com, OR 09092511083

\section{Appendix 3: Interview guide for policy makers/stakeholders}

\section{In-depth interview guide for policy makers/ stakeholders}

\section{Introduction}

\section{Section A: Interviewee background}

1. Socio-demographic characteristics of respondent

-Occupation,

- Highest educational level

- Present position

- Duration in present position

\section{Section B}

2. What are your responsibilities as the

3. How would you describe the current policy/regulation of dual practice of medical professionals in Nigeria?

4. How would you describe the benefits /disbenefits of dual practice in the public sector in Nigeria?

-Probe for any conflict of interest between public and private work due to dual practice, absences

-Probe for self-referral, quality of health care in the public and private practice managed by dual practitioners, absence, etc.

5. What mechanisms have been used to regulate or manage dual practice among public sector doctors in Nigeria?

6. What challenges have emerged from the existing mechanisms to regulate dual practice? - Probe for implementation challenges

7. What regulatory mechanisms /options would you suggest for dual practice in Nigeria, why? Any other issue relevant for DP in Nigeria?

Conclusion

Thanks

Appendix 4a: In-depth interview guide for dual practitioners (PART 1)

\section{Introduction}




\section{Section A: Interviewee background}

1. Socio-demographic characteristics of respondent

-Occupation,

- Highest educational level

- Present position

- General practice/consultant,

- Area of specialty,

- Duration in present position

\section{Section B. Major Questions}

1. Could you please describe your responsibilities here in this facility?

2. What are your views about dual practice in Nigeria? _ probe for benefits and disbenefits of dual practice (patient, DPs, public system?) - Probe whether dual practice poses a challenge to public healthcare in Nigeria

3. How would you describe your experience in the public health care system?

- Probe for challenges (number of working hours, salary, bureaucracy/administrative challenges, others? -Probe for experience in private practice (challenges working in both sectors. 4. Motives for working in both sectors? 5. How do you carry out patient referral? What factors do you consider in patient referral? - Probe - what conditions will prompt referral from private to public / and from - public to private, what are the rules for referral? What conditions might prompt self-referral? 7. What conditions would make you decide to work only for the public / private sector? - Probe for salary increase, non-financial, etc.

8. Any other issue relevant to DP in Nigeria?

Conclusion

\section{Appendix 4b: Ranking on motives for DP by dual practice doctors $\quad$ PART 2}

Please rank the following factors in order of importance from 1 to 10 where 10 is most important to you and 1 is least important to you

Control of work place - whom/ how many to treat in private practice

Avoiding bureaucracy /admin rules in public system

Income enhancement due to poor salary in the public sector

Professional development/ opportunities

Patient shortage in the public sector
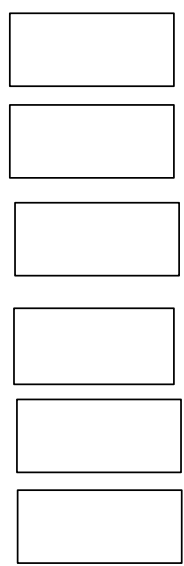
Prestige, fringe benefits and job security in the pub sector

Lucrativeness of the private sector

Not having enough hours to work in the public sector

Other (Please specify)

\section{Appendix 5: In-depth interviews guide for non-dual practitioners}

\section{Section A. Interviewee background}

1. Socio-demographic characteristics of respondent

-Occupation,

- Highest educational level

- General practice/Consultant

- Present position

- No of years in present position

- Area of specialty,

\section{B. Major questions}

1. Could you please describe your responsibilities here in this facility?

2. What are your views about dual practice in Nigeria? - Probe whether dual practice poses a challenge to public health care in Nigeria - probe for benefits and disbenefits of dual practice

3. Why do some doctors choose to work in both public and private health care simultaneously? 4. Why do some doctors like you choose not to work in both public and private sector at the same time? - Probe for reasons for not engaging in dual practice e.g. family pressure, enough income from public sector work, other secondary income generating activity apart from private practice, etc.

5. Any other issue relevant to DP in Nigeria?

Conclusion 


\section{Appendix 6: Questionnaire on dual practice of medical professionals in public}

health facilities in Nigeria

\section{Dear Respondent,}

I am a $\mathrm{PhD}$ research student from Swansea University, United Kingdom. I am in Nigeria to carry out a study on " Dual practice of medical professionals in public hospitals in Nigeria: An economic and policy analysis'. The overall aim of this study is to explore the practice when doctors work at the same time in public hospitals and private practice, so called dual practice. This practice is common in many health systems including Nigeria. However, we need to know how this practice constrains or enables health care services in public health facilities in Nigeria. The results of the research could be used to inform future policy on dual practice of public sector doctors in Nigeria.

You have been selected to complete this questionnaire because you have been identified as someone who is suitable to participate in this study.

Your participation is voluntary, and you are at liberty to bypass any question you do not wish to answer. Before we commence, I want to assure you that all information supplied in this questionnaire would be treated with utmost confidentiality during and after the study. If you have any questions, you may please ask them now or at the end of the interview. This study was approved by the Ethics Research Committee of the University of Nigeria Teaching Hospital, Ituku-Ozalla, and the Enugu State Ministry of Health, Enugu.

\section{Interview information}

This part should be filled by the investigator before the interview

Date: 1

\section{Respondent ID:}

Time interview started: Time interview ended:

\section{SECTION A: BACKGROUND INFORMATION AND CONSENT}

(Information to be filled by the investigator)

CONSENT: (Please before beginning the interview explain the consent to the respondent)

\section{Questions}

\section{Code}

\begin{tabular}{|l|l|l|}
\hline 1. & Has the respondent given consent to \\
complete the questionnaire? & & \\
Yes=1 & & \\
No=0 & \\
If yes, go to Q2, if No end the interview & \\
\hline
\end{tabular}




\begin{tabular}{|c|c|c|}
\hline 2. & $\begin{array}{l}\text { Are you currently on the National Health } \\
\text { Insurance Scheme (NHIS)? } \\
\text { Yes }=1 \\
\text { No=0 } \\
\text { If yes, end the interview; if no proceed to Q3 }\end{array}$ & ] \\
\hline 3. & $\begin{array}{l}\text { Have you or any member of your household } \\
\text { first visited a government hospital and then } \\
\text { gone to a private hospital or clinic in the last } \\
12 \text { months? } \\
\text { If Yes, go to Question number } 4 \text {, and if No } \\
\text { end the interview. } \\
\text { Yes }=1 \\
\text { No }=0\end{array}$ & $\begin{array}{l}\text { Note: Only tertiary hospitals, general } \\
\text { hospitals, polyclinics, and cottage } \\
\text { hospitals are accepted here [ ] }\end{array}$ \\
\hline 4. & $\begin{array}{l}\text { How many times have you or any member } \\
\text { of your household visited a public hospital } \\
\text { and private hospital in the last } 12 \text { months? } \\
\text { Yes }=1 \\
\text { No }=0\end{array}$ & $\begin{array}{l}\text { visit to government hospital does not } \\
\text { include primary health care centres or } \\
\text { maternity homes } \\
\text { Insert number of times in words } \\
\text { 4a. Public hospital [ ] no of times[ } \\
\text { ] } \\
\text { 4b. Private hospital [ ] no of times[ } \\
\text { ] }\end{array}$ \\
\hline
\end{tabular}

\section{SECTION B: SOCIO-DEMOGRAPHIC DETAILS OF RESPONDENT}

In this section, we would like to ask you some questions about you and your household

\section{Questions}

Code

\begin{tabular}{|c|c|c|}
\hline 5. & Sex of respondent? & $\begin{array}{l}\text { 1=Male } \\
2=\text { Female } \quad[\quad]\end{array}$ \\
\hline 6. & $\begin{array}{l}\text { What is your status in your household? } \\
\text { (insert number code only) } \\
1=\text { Yes } \\
0=\text { No } \\
\text { (One 'Yes' response only) }\end{array}$ & $\begin{array}{l}\text { 6a. Male head of household [ ] } \\
\text { 6b. Female head of household [ ] } \\
\text { 6c. Son/Daughter [ ] } \\
\text { 6d. Refuse to answer [ ] } \\
\text { 6e. Other [ ] } \\
\text { Please Specify }\end{array}$ \\
\hline 7. & $\begin{array}{l}\text { How many are in your household } \\
\text { including you }\end{array}$ & {[} \\
\hline 8 & What is your age at last birthday? & ] years \\
\hline 9. & What is your marital status & \\
\hline
\end{tabular}




\begin{tabular}{|c|c|c|}
\hline & $\begin{array}{l}\text { (insert code number only) } \\
1=\text { Yes } \\
\text { No=0 } \\
\text { (one 'Yes' response only) }\end{array}$ & 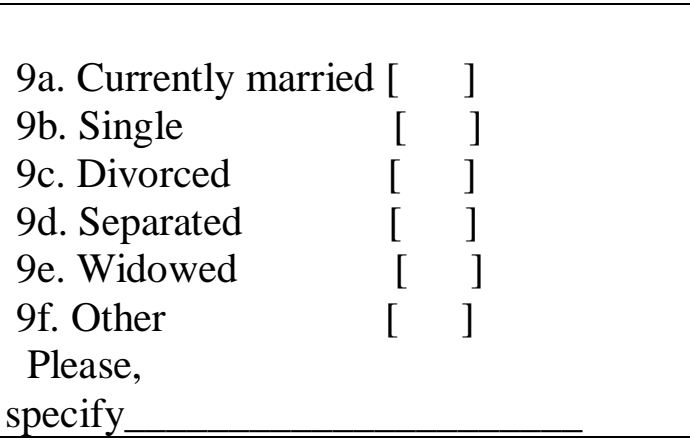 \\
\hline 10. & $\begin{array}{l}\text { Did you have a formal education? } \\
\text { Yes=1 } \\
\text { No=0 } \\
\text { If yes go to Q11 if No go to Q12 }\end{array}$ & ] \\
\hline 11. & $\begin{array}{l}\text { What was the highest level of education } \\
\text { completed } \\
\text { (Insert code number only) } \\
\text { Yes }=1 \\
\text { No }=0 \\
\text { (One 'Yes' response only) }\end{array}$ & $\begin{array}{l}\text { 11a. Primary school [ ] } \\
\text { 11b. Junior Secondary [ ] } \\
\text { 11c. Senior Secondary school [ ] } \\
\text { 11d. Ordinary National Diploma [ ] } \\
\text { 11e. Higher National Diploma [ ] } \\
\text { 11f. Bachelor degree [ ] } \\
\text { 11g. MSc and above [ ] } \\
\text { 11h. Other [ ] } \\
\text { Please specify }\end{array}$ \\
\hline 12. & $\begin{array}{l}\text { What occupational group is your major } \\
\text { source of income? } \\
\text { (Insert code number only) } \\
\text { Yes }=1 \\
\text { No=0 } \\
\text { (One 'Yes' response only) }\end{array}$ & $\begin{array}{l}\text { 12a. Government worker [ ] } \\
\text { 12b. Employed in the private sector[ ] } \\
\text { 12c. Self-employed [ ] } \\
\text { 12d. Artisan/petty trading [ ] } \\
\text { 12e. Student [ ] } \\
\text { 12f. Unemployed [ ] } \\
\text { 12g. Other [ ] } \\
\text { Please specify }\end{array}$ \\
\hline
\end{tabular}

\section{SECTION C: HOSPITAL VISIT EXPERIENCES AND EXTENT OF SELF- REFERRAL}

In this section I will ask you about your hospital visit experiences and how you have been referred from a government hospital to a private hospital

\begin{tabular}{|l|l|l|}
\hline 13. & $\begin{array}{l}\text { What made you or a member of your } \\
\text { household to decide visiting a } \\
\text { government hospital FIRST } \\
\text { Yes }=1\end{array}$ & $\begin{array}{l}\text { Refer to Q3 } \\
\text { 13a. Lower cost of care in government } \\
\text { hospital [ ] } \\
\text { (One 'Yes' response only) }\end{array}$ \\
& $\begin{array}{l}\text { 13b. Many specialists in government } \\
\text { hospital [ ] } \\
\text { 13c. Availability of health staff at all-time } \\
\text { [ ] } \\
\text { 13d. Proximity of government hospital to } \\
\text { your home [ ] } \\
\text { 13e. A family member / friend influenced }\end{array}$ \\
\hline
\end{tabular}




\begin{tabular}{|c|c|c|}
\hline & & $\begin{array}{l}\text { me to attend the government hospital [ ] } \\
\text { 13f.Other, } \quad \text { please } \quad \text { specify }\end{array}$ \\
\hline 14. & $\begin{array}{l}\text { Was your visit or that of a member of } \\
\text { your household to a private hospital } \\
\text { for the same health problem that first } \\
\text { took you to a public hospital? } \\
\text { Yes }=1 \\
\text { No=0 }\end{array}$ & {[} \\
\hline 15. & $\begin{array}{l}\text { How sure are you that the doctor who } \\
\text { treated you or a member of your } \\
\text { household in the private hospital or } \\
\text { clinic also works in a government } \\
\text { hospital? } \\
\text { Yes }=1 \\
\text { No=0 } \\
\text { (One 'Yes' response only) }\end{array}$ & $\begin{array}{l}\text { 15a. Certainly sure [ ] } \\
\text { 15b. Not too sure [ ] } \\
\text { 15c. Don't know [ ] }\end{array}$ \\
\hline 16. & $\begin{array}{l}\text { Have you or a member of your } \\
\text { household been referred from a } \\
\text { government hospital to a private } \\
\text { hospital/clinic by a health worker in } \\
\text { the government hospital? } \\
\text { Yes }=1 \\
\text { No=0 } \\
\text { If yes, Go to Q17 If no GO to section } \\
\mathbf{E}\end{array}$ & $\begin{array}{l}\text { Only those referred by a health worker } \\
\text { that works in the government hospital can } \\
\text { answer this question } \\
\text { Note: formal and informal referral } \\
\text { [ ] }\end{array}$ \\
\hline 17. & $\begin{array}{l}\text { Who referred you or a member of your } \\
\text { household from a government hospital } \\
\text { to a private hospital/clinic? } \\
\text { (insert number codes) } \\
\text { Yes }=1 \\
\text { No }=0 \\
\text { (One 'Yes' response only) }\end{array}$ & $\begin{array}{l}\text { 17a. A doctor [ ] } \\
\text { 17b. A nurse [ ] } \\
\text { 17c. Other [ ] } \\
\text { Please Specify }\end{array}$ \\
\hline 18 & $\begin{array}{l}\text { How many times have you or a } \\
\text { member of your household been } \\
\text { referred from a government hospital to } \\
\text { a private hospital/ clinic? } \\
\text { Yes }=1 \\
\text { No=0 } \\
\text { (One 'Yes' response only) }\end{array}$ & $\begin{array}{l}\text { 18a. Once [ ] } \\
\text { 18b. Twice [ ] } \\
\text { 18c. Thrice [ ] } \\
\text { 18d. More than four times [ ] }\end{array}$ \\
\hline 19. & $\begin{array}{l}\text { How were you or a member of your } \\
\text { household referred from a government } \\
\text { hospital to a private hospital/clinic? } \\
\text { Yes }=1 \\
\text { No=0 }\end{array}$ & $\begin{array}{l}\text { 19a. Doctor spoke to me and advised me to } \\
\text { go to a particular private hospital/clinic } \\
\text { ] } \\
\text { 19b.Doctor handed over his or her } \\
\text { business card to me [ ] }\end{array}$ \\
\hline
\end{tabular}




\begin{tabular}{|c|c|c|}
\hline & $\begin{array}{l}\text { (Insert code number only) } \\
\quad \text { Multiple answers are allowed }\end{array}$ & $\begin{array}{l}\text { 19c.Doctor gave me a mobile phone } \\
\text { number to call [ ] } \\
\text { 19d.Advised by a nurse to visit a specific } \\
\text { private hospital/clinic in town [ ] } \\
\text { 19e.Doctor gave me a referral letter [ ] } \\
\text { 19f.Other [ ] } \\
\text { Please specify_ }\end{array}$ \\
\hline 20. & $\begin{array}{l}\text { For what type of health problem were } \\
\text { you or a member of your household } \\
\text { referred from a government hospital to } \\
\text { a private hospital/clinic? } \\
\text { (Insert code number only) } \\
\text { Yes }=1 \\
\mathrm{No}=0 \\
\text { Multiple answers are allowed }\end{array}$ & $\begin{array}{l}\text { 20a. Malaria [ ] } \\
\text { 20b. Childhood diseases [ ] } \\
\text { 20c. Heart disease [ ] } \\
\text { 20e. Respiratory disease [ ] } \\
\text { 20d. Pregnancy /child delivery related [ ] } \\
\text { 20f. Eye problem [ ] } \\
\text { 20g. Surgery [ ] } \\
\text { 20h. Stroke [ ] } \\
\text { 20i. Don't know [ ] } \\
\text { 20j. Refuse to say [ ] } \\
\text { 20k. Other [ ] } \\
\text { Please } \\
\text { specify }\end{array}$ \\
\hline 21. & $\begin{array}{l}\text { Was the illness an emergency case? } \\
\text { Yes }=1 \\
\text { No }=0\end{array}$ & P \\
\hline 22. & $\begin{array}{l}\text { Were you or a member of your } \\
\text { household admitted as an inpatient or } \\
\text { outpatient to a private hospital when } \\
\text { you or your household member was } \\
\text { referred from a government hospital? } \\
\text { Yes }=1 \\
\text { No }=0 \text { (One 'Yes' response only) }\end{array}$ & $\begin{array}{l}\text { 22a. Inpatient [ ] } \\
\text { 22b. Outpatient [ ] }\end{array}$ \\
\hline 23. & $\begin{array}{l}\text { Did a health worker in the government } \\
\text { hospital seek your opinion on whether } \\
\text { to be treated in the government } \\
\text { hospital or to move you to a private } \\
\text { hospital/clinic before you were } \\
\text { referred from the government } \\
\text { hospital? } \\
\text { Yes }=1, \text { No=0 } \\
\text { If yes Go to Q24, if no Go to Q25 }\end{array}$ & ] \\
\hline 24. & $\begin{array}{l}\text { What reason (s) was given for } \\
\text { referring you or a member of your } \\
\text { household from the government to a } \\
\text { private hospital/clinic? } \\
\text { Yes }=1, \mathrm{No}=0\end{array}$ & $\begin{array}{l}\text { 24a. Lack of equipment in government } \\
\text { Hospital [ ] } \\
\text { 24b.Lack of specialists in government } \\
\text { Hospital [ ] }\end{array}$ \\
\hline
\end{tabular}




\begin{tabular}{|c|c|c|}
\hline & (Insert code number only) & $\begin{array}{l}\text { 24c. Emergency case that needed urgent } \\
\text { attention [ ] } \\
\text { 24d. No reason was given [ ] } \\
\text { 24e. Other [ ] } \\
\text { Please specify }\end{array}$ \\
\hline 25. & $\begin{array}{l}\text { How does your referral from a } \\
\text { government hospital to private } \\
\text { hospital/clinic deprive you of services } \\
\text { you receive in government hospital? }\end{array}$ & $\begin{array}{l}\text { 25a. Deprives me of lower cost of care in } \\
\text { government hospital [ ] } \\
\text { 25b. Deprives me of some free medical } \\
\text { services in government hospital [ ] } \\
\text { 25c. Not having access to specialists in } \\
\text { government hospital [ ] } \\
\text { 25d. Makes me to avoid government } \\
\text { hospital [ ] } \\
\text { 26e. Other [ ] } \\
\text { Please } \\
\text { specify }\end{array}$ \\
\hline
\end{tabular}

\section{SECTION D: COSTS INCURRED AS A RESULT OF SELF-REFERRAL}

In this section, I will ask you about the costs you or your household incurred when you were referred from a government hospital to a private one?

26. Read out the different items in the cost category to the respondent and record the cost of each item in naira (Investigator, refer the respondent to Q20)

\begin{tabular}{|c|c|c|}
\hline & Category of cost & Amount in Naira \\
\hline $26 \mathrm{a}$ & Transport going to the private hospital & \\
\hline $26 \mathrm{~b}$ & Transport return from the private hospital & \\
\hline $26 \mathrm{c}$. & Cost of hospital card/registration & \\
\hline $26 \mathrm{~d}$ & Consultation fee & \\
\hline $26 \mathrm{e}$ & Cost of drugs & \\
\hline $26 f$ & Cost of laboratory test & \\
\hline $26 \mathrm{~g}$ & Cost of X-ray if any & \\
\hline \multirow[t]{2}{*}{$26 \mathrm{~h}$} & $\begin{array}{l}\text { Other } \\
\text { Please }\end{array}$ & \\
\hline & & Total cost (Add up) [ \\
\hline \multirow[t]{2}{*}{27.} & $\begin{array}{l}\text { If you or any member of your household were } \\
\text { to receive the same treatment for the same } \\
\text { health problem in a government hospital, how } \\
\text { much would it cost? }\end{array}$ & \\
\hline & & Amount in Naira \\
\hline
\end{tabular}




\begin{tabular}{|c|c|c|}
\hline & Category of costs & \\
\hline $27 \mathrm{a}$ & Transport going to the government hospital & \\
\hline $27 \mathrm{~b}$ & Transport return from government hospital & \\
\hline $27 \mathrm{c}$ & Cost of hospital card/registration & \\
\hline $27 d$ & Consultation fee & \\
\hline $27 \mathrm{e}$ & Cost of drugs & \\
\hline $27 f$ & Cost of laboratory test & \\
\hline $27 \mathrm{~g}$ & Cost of X-ray if any & \\
\hline $27 \mathrm{~h}$ & $\begin{array}{l}\text { Other } \\
\text { Specify }\end{array}$ & Total costs Add up [ \\
\hline 28. & $\begin{array}{l}\text { What mode of payment did you or a member } \\
\text { of your household use to pay for services } \\
\text { received in the private hospital? } \\
\text { Yes }=1, \mathrm{No}=0 \\
\text { (One 'Yes' response only) }\end{array}$ & $\begin{array}{l}\text { 28a. Cash [ ] } \\
\text { 28b. Instalment payment [ ] } \\
\text { 28c. In kind [ ] } \\
\text { 28d. Other [ ] } \\
\text { Please } \\
\text { specify }\end{array}$ \\
\hline 29. & $\begin{array}{l}\text { How did you or a member of your household } \\
\text { source the funds to pay for services received } \\
\text { from the private hospital/clinic? } \\
\text { (insert number codes only) } \\
\text { Yes }=1, \mathrm{No}=0 \\
\text { (Multiple answers are allowed) }\end{array}$ & $\begin{array}{l}\text { 29a. Own money [ ] } \\
\text { 29b. Borrowed money/took Loan[ ] } \\
\text { 29c. Sold household movable assets } \\
\text { [ ] } \\
\text { 29d. Sold family land [ ] } \\
\text { 29e. Someone else paid [ ] } \\
\text { 29f. Bill was reduced by the doctor } \\
\text { [ ] } \\
\text { 29g. Other [ ] } \\
\text { Please specify }\end{array}$ \\
\hline 30. & $\begin{array}{l}\text { To what extent has your referral or that of a } \\
\text { member of your household from a } \\
\text { government hospital to a private } \\
\text { hospital/clinic affected your household } \\
\text { financially? } \\
\text { Yes=1, No=0 (One 'Yes' response only) }\end{array}$ & $\begin{array}{l}\text { 30a. No impact [ ] } \\
\text { 30b. Moderate impact [ ] } \\
\text { 30c. Serious impact [ ] } \\
\text { 30d. Very serious impact [ ] }\end{array}$ \\
\hline
\end{tabular}

\section{SECTION E: SERVICE USERS' PERCEPTONS OF PUBLIC SECTOR DOCTORS WHO ENGAGE IN PRIVATE PRACTIC}

In this section I want to ask you what you think about public sector doctors who also engage in private practice

31. What do you think about the following statements? (Please tick one box for each statement) 


\begin{tabular}{|c|c|c|c|c|c|}
\hline & $\begin{array}{c}\text { Strongly } \\
\text { disagree } \\
1\end{array}$ & $\begin{array}{c}\text { Disagree } \\
2\end{array}$ & $\begin{array}{l}\text { Don't } \\
\text { know } \\
3\end{array}$ & $\begin{array}{c}\text { Agree } \\
4\end{array}$ & $\begin{array}{c}\text { Strongly } \\
\text { agree } \\
5\end{array}$ \\
\hline $\begin{array}{l}\text { 31a). Doctors employed in } \\
\text { government hospitals engage } \\
\text { in private practice for their } \\
\text { own financial gains }\end{array}$ & & & & & \\
\hline $\begin{array}{l}\text { 31b). Doctors employed in } \\
\text { government hospitals engage } \\
\text { in private practice to raise their } \\
\text { poor salary }\end{array}$ & & & & & \\
\hline $\begin{array}{l}\text { 31c). Strikes in government } \\
\text { hospitals encourage public } \\
\text { hospital patients to move to } \\
\text { private hospitals where } \\
\text { government doctors also work }\end{array}$ & & & & & \\
\hline $\begin{array}{l}\text { 31d). Delays in paying public } \\
\text { hospital doctors' salaries make } \\
\text { them engage in private practice }\end{array}$ & & & & & \\
\hline $\begin{array}{l}\text { 31e). Lack of equipment in } \\
\text { government hospitals make } \\
\text { government doctors engage in } \\
\text { private practice }\end{array}$ & & & & & \\
\hline $\begin{array}{l}\text { 31f). Government doctors } \\
\text { engage in private practice } \\
\text { because government hospitals } \\
\text { are poorly supervised and } \\
\text { monitored }\end{array}$ & & & & & \\
\hline $\begin{array}{l}\text { 31g). Please specify any other } \\
\text { factor that you believe make } \\
\text { doctors engage in dual } \\
\text { practice. }\end{array}$ & & & & & \\
\hline
\end{tabular}

\section{BENEFITS AND DISBENEFITS OF PRIVATE PRACTICE BY PUBLIC SECTOR DOCTORS}

32. I would like to ask you what you think are the benefits to you and your household of government doctors engaged in private practice in Nigeria 


\begin{tabular}{|c|c|c|c|c|c|}
\hline & $\begin{array}{c}\text { Strongly } \\
\text { disagree } \\
1\end{array}$ & $\begin{array}{c}\text { Disagree } \\
2\end{array}$ & $\begin{array}{c}\text { Don't } \\
\text { know } \\
3\end{array}$ & $\begin{array}{c}\text { Agree } \\
4\end{array}$ & $\begin{array}{c}\text { Strongly } \\
\text { agree } \\
5\end{array}$ \\
\hline \multicolumn{6}{|l|}{$\begin{array}{l}\text { 32a). Government doctors give } \\
\text { speedier attention to patients in } \\
\text { their private practice than in } \\
\text { government hospital }\end{array}$} \\
\hline \multicolumn{6}{|l|}{$\begin{array}{l}\text { 32b). Access to government } \\
\text { doctors in government hospital } \\
\text { is quicker than in their private } \\
\text { practice }\end{array}$} \\
\hline \multicolumn{6}{|l|}{$\begin{array}{l}\text { 32c). Government doctors } \\
\text { engaged in private practice } \\
\text { have more functioning medical } \\
\text { equipment than those in } \\
\text { government hospitals }\end{array}$} \\
\hline \multicolumn{6}{|l|}{$\begin{array}{l}\text { 32d). There is a better quality } \\
\text { of care when treated by a } \\
\text { government doctor in a } \\
\text { government hospital than in his } \\
\text { private practice }\end{array}$} \\
\hline \multicolumn{6}{|l|}{$\begin{array}{l}\text { 32e). Government doctors } \\
\text { engaged in private practice use } \\
\text { their private practice to save } \\
\text { lives during strikes in } \\
\text { government hospitals }\end{array}$} \\
\hline \multicolumn{6}{|l|}{$\begin{array}{l}\text { 32f). There is quicker access to } \\
\text { a specialist in his private } \\
\text { practice than in his government } \\
\text { hospital }\end{array}$} \\
\hline \multicolumn{6}{|l|}{$\begin{array}{l}\text { 32g). Instalment payment may } \\
\text { be allowed in a private practice } \\
\text { than in government hospital }\end{array}$} \\
\hline \multicolumn{6}{|l|}{$\begin{array}{l}\text { 32h). There is a shorter wait } \\
\text { time to see a government doctor } \\
\text { in a government hospital than } \\
\text { in his own private practice }\end{array}$} \\
\hline \multicolumn{6}{|l|}{$\begin{array}{l}\text { 32i). There is less bureaucracy } \\
\text { (protocol) in private practice } \\
\text { than } \\
\text { in the government hospital }\end{array}$} \\
\hline $\begin{array}{l}32 \mathbf{j} \text { ). Patient/ doctor } \\
\text { relationship is stronger in a } \\
\text { government hospital than in a } \\
\text { private practice }\end{array}$ & & & & & \\
\hline
\end{tabular}




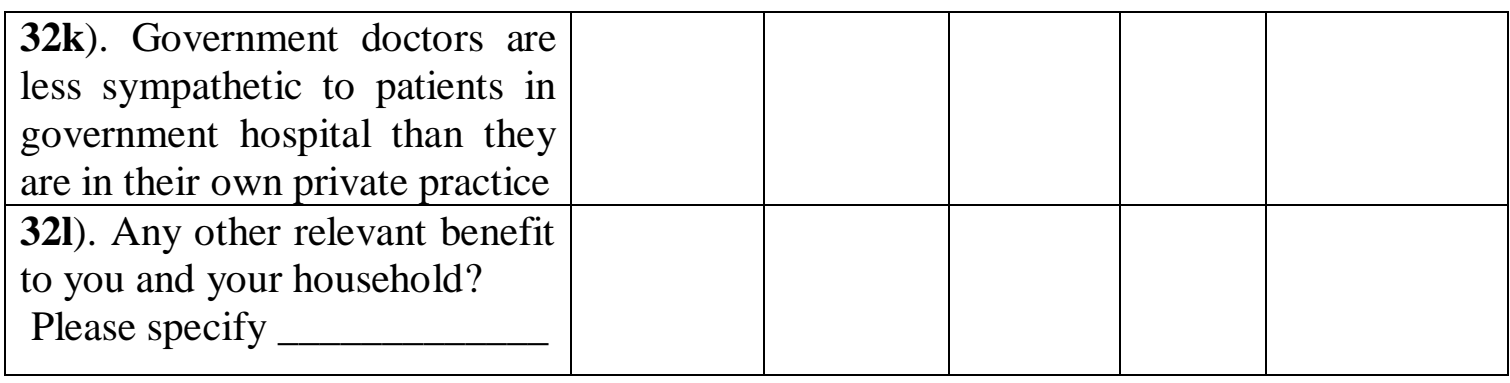

33. Here, I want to know what you think are the disbenefits/disadvantages to you and your household of government doctors engaged in private practice in Nigeria

\begin{tabular}{|c|c|c|c|c|c|}
\hline & $\begin{array}{c}\text { Strongly } \\
\text { disagree } \\
1\end{array}$ & $\begin{array}{c}\text { Disagree } \\
2\end{array}$ & $\begin{array}{c}\text { Don't } \\
\text { know } \\
3\end{array}$ & $\begin{array}{c}\text { Agree } \\
4\end{array}$ & $\begin{array}{c}\text { Strongly agree } \\
5\end{array}$ \\
\hline $\begin{array}{l}\text { 33a). Government doctors } \\
\text { engaged in private practice } \\
\text { come late to work in } \\
\text { government hospital }\end{array}$ & & & & & \\
\hline $\begin{array}{l}\text { 33b). Government doctors } \\
\text { engaged in private practice are } \\
\text { usually absent from work in } \\
\text { government hospital }\end{array}$ & & & & & \\
\hline $\begin{array}{l}\text { 33c). Doctors engaged in dual } \\
\text { practice divert patients from the } \\
\text { government hospital to their } \\
\text { private practice }\end{array}$ & & & & & \\
\hline $\begin{array}{l}\text { 33d). Government doctors } \\
\text { engaged in private practice give } \\
\text { poor service to patients in } \\
\text { government hospitals }\end{array}$ & & & & & \\
\hline $\begin{array}{l}\text { 33e). Cost of care is not } \\
\text { affordable in private practice of } \\
\text { government doctors }\end{array}$ & & & & & \\
\hline $\begin{array}{l}\text { 33f). Government doctors spend } \\
\text { shorter time to see patients in a } \\
\text { government hospital than in } \\
\text { their private practice }\end{array}$ & & & & & \\
\hline $\begin{array}{l}\text { 33g). Government doctors first } \\
\text { attend to patients in their private } \\
\text { practice before coming to } \\
\text { government hospital }\end{array}$ & & & & & \\
\hline $\begin{array}{l}\text { 33h). Any other relevant } \\
\text { disadvantage to you and your } \\
\text { household } \\
\text { please specify }\end{array}$ & & & & & \\
\hline
\end{tabular}




\section{SECTION F: COMPARING QUALITY OF PUBLIC HEALTH CARE AND PRIVATE HEALTH PRACTITIONERS}

34. In this section, I would want you to recall your hospital visit experiences in both public hospitals anc government doctors also work and respond to the questions below. Please read the statements below and inc with them. $1=$ Strongly Disagree, $2=$ Disagree, 3= Don't Know, $4=$ Agree, $5=$ Strongly Agree (tick one box o

\begin{tabular}{|c|c|c|}
\hline $\begin{array}{l}\text { Structural element of quality } \\
\text { (Physical attributes where care takes place) }\end{array}$ & $\begin{array}{c}\text { Strongly } \\
\text { disagree } \\
1 \\
\end{array}$ & $\begin{array}{l}\text { Disagree } \\
2\end{array}$ \\
\hline \multicolumn{3}{|l|}{$\begin{array}{l}\text { 34a. Better physical infrastructure (building) exists in public hospitals than in } \\
\text { private practices set up by government doctors }\end{array}$} \\
\hline \multicolumn{3}{|l|}{$\begin{array}{l}\text { 34b. There is better availability of equipment in public hospitals than in } \\
\text { private practices of government doctors }\end{array}$} \\
\hline \multicolumn{3}{|l|}{$\begin{array}{l}\text { 34c. There are fewer qualified health care staff in private practices of } \\
\text { government doctors compared with public facilities }\end{array}$} \\
\hline \multicolumn{3}{|l|}{$\begin{array}{l}\text { 34d. Government doctors engaged in private practice have better opening } \\
\text { and closing hours than public hospitals }\end{array}$} \\
\hline \multicolumn{3}{|l|}{$\begin{array}{l}\text { 34e. There is better availability of drugs in private practice of government } \\
\text { doctors than in government hospitals }\end{array}$} \\
\hline \multicolumn{3}{|l|}{$\begin{array}{l}\text { Process element of quality } \\
\text { (What is actually done in the process of receiving care) }\end{array}$} \\
\hline $\begin{array}{l}\text { 34f. The waiting time to see a doctor in public hospitals is less than the } \\
\text { waiting time to see him in his private practice }\end{array}$ & & \\
\hline
\end{tabular}




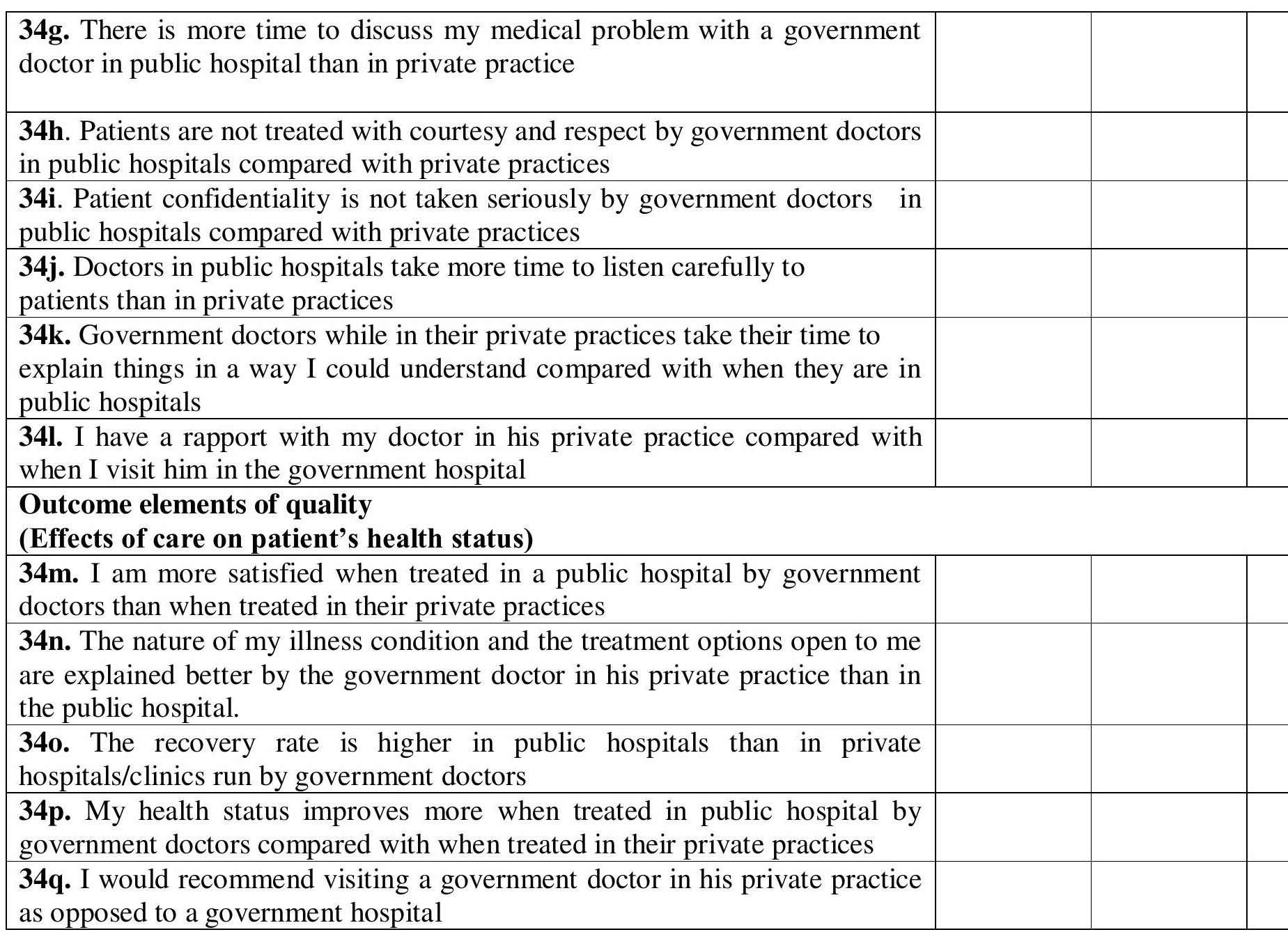




\section{SECTION G: HOUSEHOLD ASSET HOLDING AND LIVING CONDITIONS}

I will now ask you about some of the items your household owns. This will help us to determine your socio-economic status.

\begin{tabular}{|c|c|c|c|}
\hline \multirow[t]{15}{*}{35.} & \multicolumn{3}{|c|}{$\begin{array}{l}\text { Could you please tell me if your household has any of the following functional items? } \\
\text { Yes }=1, \mathrm{No}=0\end{array}$} \\
\hline & & Item & Present and functional \\
\hline & $35 \mathrm{a}$ & Bicycle & {$[\quad]$} \\
\hline & $35 b$ & Radio & ] \\
\hline & $35 c$ & Refrigerator/Freezer & ] \\
\hline & $35 \mathrm{~d}$ & Colour Television & ] \\
\hline & $35 \mathrm{e}$ & Gas cooker & ] \\
\hline & $35 f$ & Motorcycle & ] \\
\hline & $35 \mathrm{~g}$ & Car & ] \\
\hline & $35 \mathrm{~h}$ & Generator & ] \\
\hline & $35 \mathrm{i}$ & Electric Iron & ] \\
\hline & $35 \mathrm{j}$ & Electric fan & ] \\
\hline & $35 \mathrm{k}$ & Air-conditioner & ] \\
\hline & 351 & A plot of land in a city & ] \\
\hline & $35 \mathrm{~m}$ & Satellite dish & ] \\
\hline 36. & $\begin{array}{l}\text { What is } \\
\text { drinking } \\
\text { Yes }=1 \\
\text { No=0 } \\
\text { (One Yes }\end{array}$ & $\begin{array}{l}\text { our household's main source of } \\
\text { ater? } \\
\text { response only) }\end{array}$ & $\begin{array}{l}\text { 36a. Tap water [ ] } \\
\text { 36b. Bore hole [ ] } \\
\text { 36c. Dug well [ ] } \\
\text { 36d. Rainwater [ ] } \\
\text { 36e. Surface water (river/ stream) [ } \\
\text { 36f. packaged sachet [ ] } \\
\text { 36g. Refuse to answer [ ] } \\
\text { 36h. Other [ ] } \\
\text { Specify }\end{array}$ \\
\hline 37. & \multicolumn{2}{|c|}{$\begin{array}{l}\text { What kind of toilet facility do you have in } \\
\text { your home? } \\
\text { Yes }=1 \\
\text { No=0 } \\
\text { (One Yes response only) }\end{array}$} & $\begin{array}{l}\text { 37a. Shared flush toilet (water system) [ } \\
\text { ] } \\
\text { 37b. Private flush toilet [ ] } \\
\text { 37c. Shared pit latrine [ ] } \\
\text { 37d Private pit latrine [ ] } \\
\text { 37e. Bush [ ] } \\
\text { 37f. Refuses to answer [ ] } \\
\text { 37g Other [ ] } \\
\text { Please }\end{array}$ \\
\hline 38. & \multicolumn{2}{|c|}{$\begin{array}{l}\text { What type of fuel does your household } \\
\text { mainly use for cooking? } \\
\text { Yes }=1, \mathrm{No}=0\end{array}$} & 38a. Electricity [ ] \\
\hline
\end{tabular}




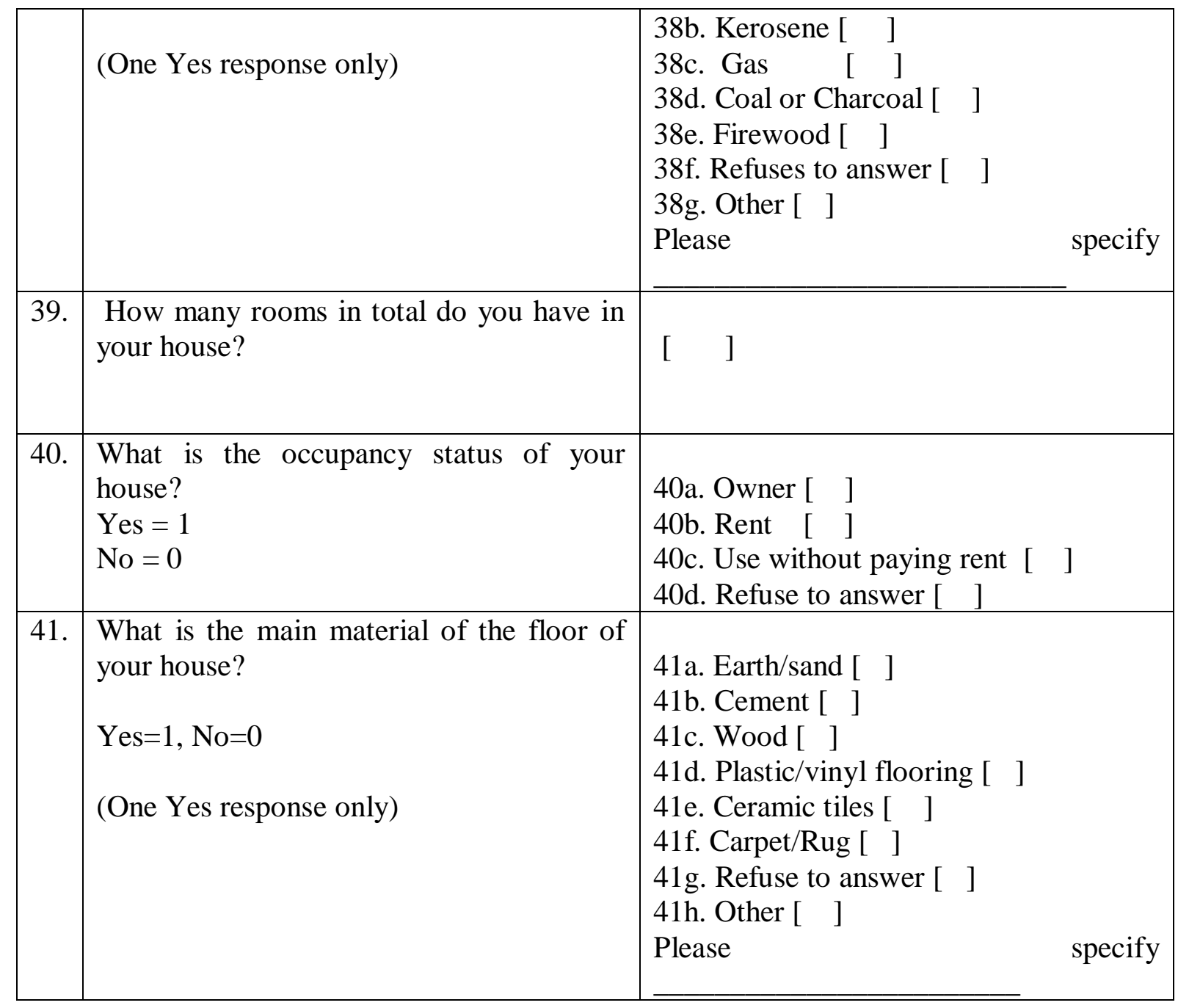

\section{SECTION H: EXPENDITURE ON FOOD}

This section will be used to obtain information that will help to determine your socio-economic status. Therefore, I will ask you a few questions on how much your household spends on food and other supplies.

42. Please could you tell me whether you and your household consumed these items in the past week and how much they spent on them? (Record only the food items that were purchased and consumed in the past week. You may consult other members of the household to get accurate expenses when necessary).

\begin{tabular}{|l|l|l|}
\hline Items & Quantity bought & $\begin{array}{l}\text { Cost (Amount in Naira) If } \\
\text { cost is not known insert 00 }\end{array}$ \\
\hline 42a. Garri & & \\
\hline 42b. Beans & & \\
\hline 42c. Cassava (Akpu) & & \\
\hline 42d. Rice & & \\
\hline 42e. Yam & & \\
\hline
\end{tabular}




\begin{tabular}{|l|l|l|}
\hline 42f. Fish & & \\
\hline 42g. Meat & & \\
\hline 42h. Vegetables & & \\
\hline 42i Cooking oil & & \\
\hline 42j. Other, specify & & \\
\hline 42k. Grand total & Add total food costs [ ] \\
\hline
\end{tabular}

43. If the food items that your household produced and consumed in the past one week were bought from the market, how much did they cost? (If no item is produced, rule the table and write NOT applicable)

\begin{tabular}{|l|l|l|}
\hline Items & Quantity bought & $\begin{array}{l}\text { Cost Amount in Naira) If } \\
\text { cost is not known insert 00 }\end{array}$ \\
\hline 43a. Garri & & \\
\hline 43b. Beans & & \\
\hline 43c. Cassava (Akpu) & & \\
\hline 43d. Rice & & \\
\hline 43e. Yam & & \\
\hline 43f. Fish & & \\
\hline 43g. Meat & & \\
\hline 43h. Vegetables & & \\
\hline 43i Cooking oil & & Add total food costs \\
\hline 43j. Other, specify & & [ ] \\
\hline 43k. Grand total & & \\
\hline
\end{tabular}

44. Total food value (Add 42k+43k)

Thank You 
Appendix 7: Visual representation of a theme on perception of quality healthcare (Focus group dis

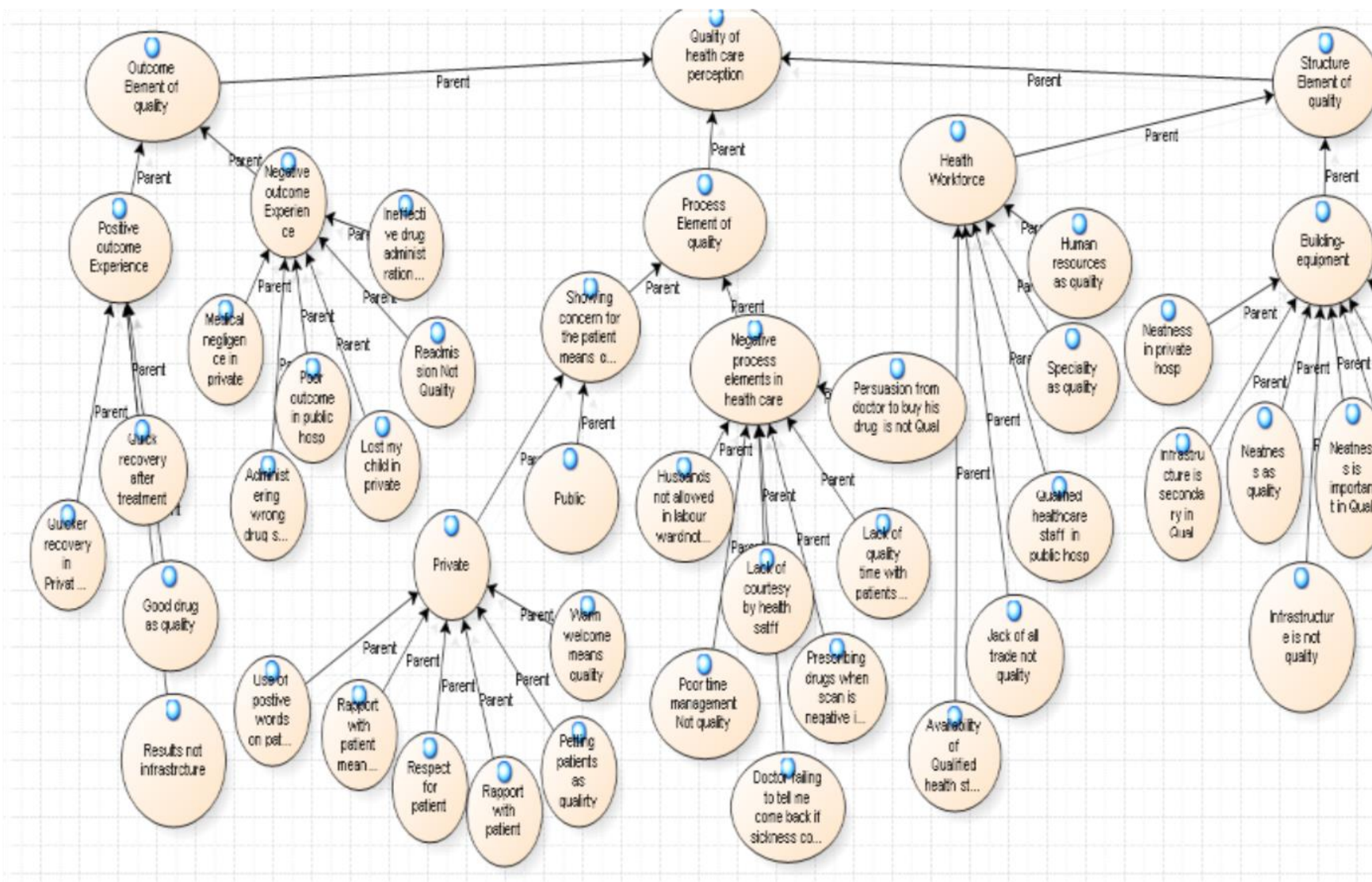


Appendix 8: Visual representation of a theme on intramural practice (policy makers' interview)

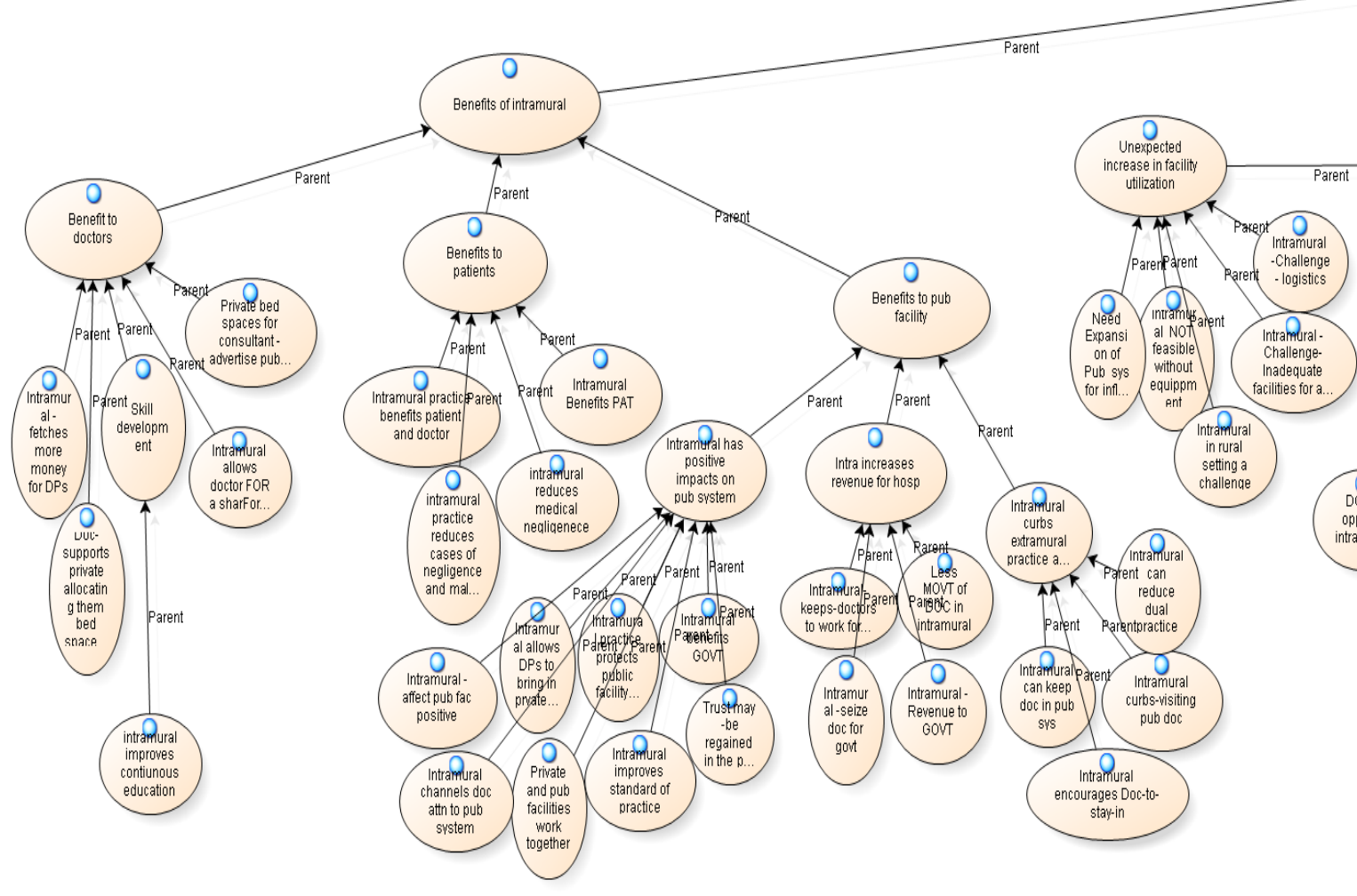


Appendix 9: Visual representation of a theme on motives for dual practice (doctors' interviews)

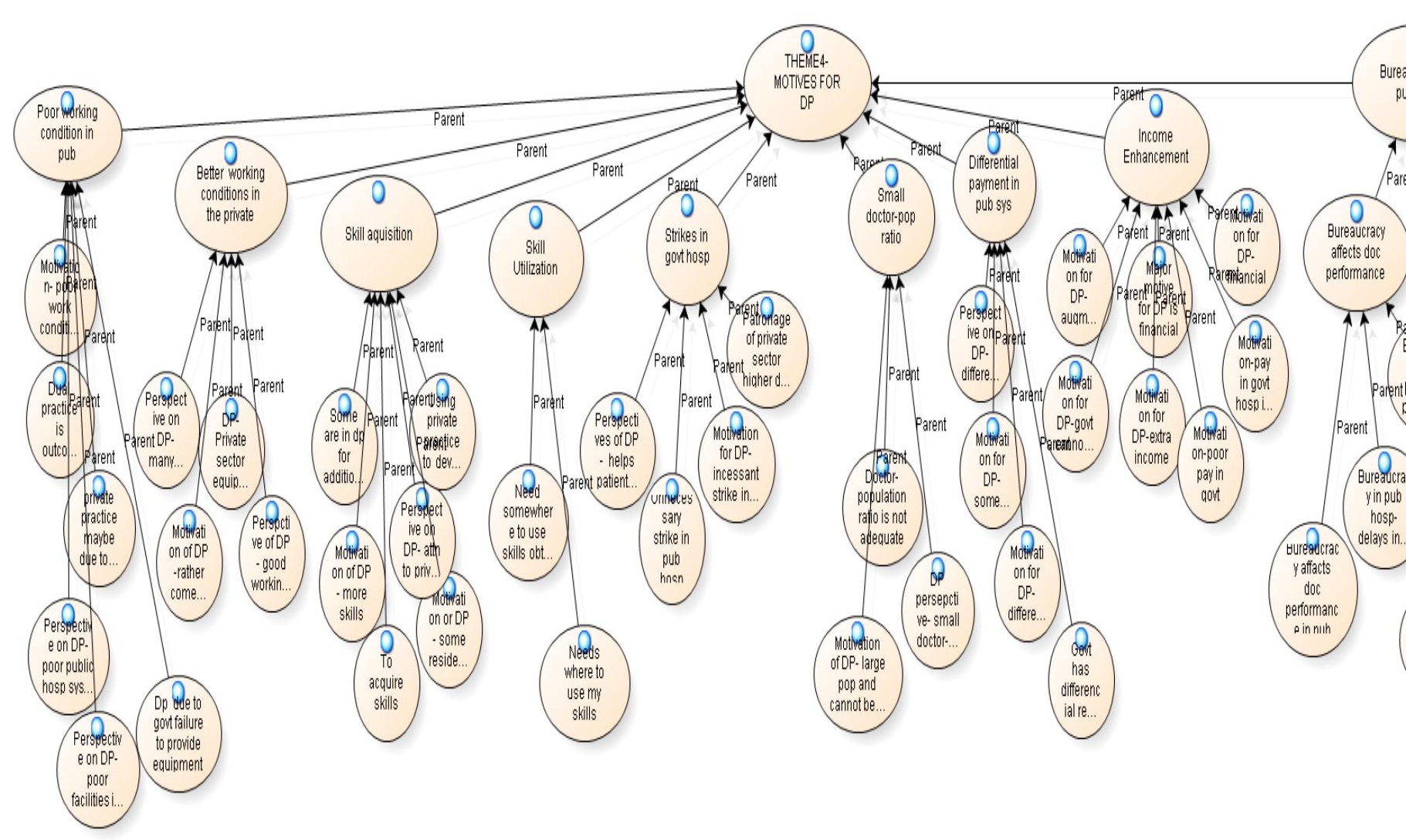




\section{Appendix 10: Consolidated Medical Salary Structure (doctors' salary with other a}

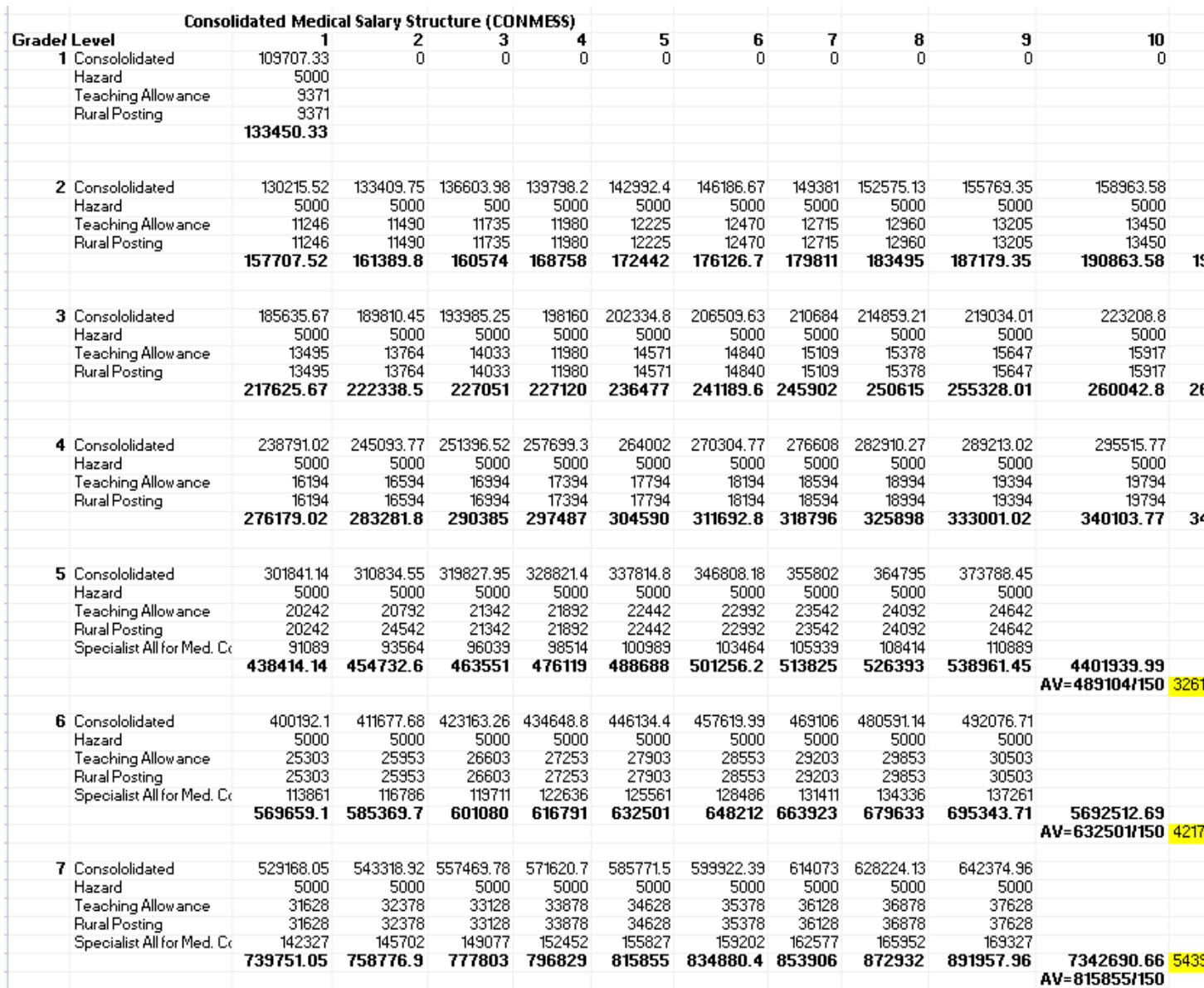


Appendix 11: Computation of different hypothetical scenarios using Excel Worksheet (Example)

\begin{tabular}{|l|l|l|l|l|l|l|l|l|l|l|}
\hline $\begin{array}{l}\text { Grade } \\
\text { Levels }\end{array}$ & $\begin{array}{l}\text { Basic } \\
\text { Hours }\end{array}$ & $\begin{array}{l}\text { AV } \\
\text { monthly } \\
\text { salary }\end{array}$ & $\begin{array}{l}\text { AV. } \\
\text { monthl } \\
\text { yrs } \\
(150)\end{array}$ & $\begin{array}{l}\text { hourly } \\
\text { pay }\end{array}$ & $\begin{array}{l}\text { Hours } \\
\text { lost } \\
\text { per } \\
\text { week }\end{array}$ & $\begin{array}{l}\text { Value } \\
\text { of } \\
\text { hours } \\
\text { lost } \\
\text { per } \\
\text { week } \\
(5)\end{array}$ & $\begin{array}{l}\text { Value of } \\
\text { hours } \\
\text { lost per } \\
\text { year }(5)\end{array}$ & $\begin{array}{l}\text { Value of } \\
\text { hours } \\
\text { lost per } \\
\text { year } \\
\text { @ 10\% } \\
\text { of DP }\end{array}$ & $\begin{array}{l}\text { Value } \\
\text { of } \\
\text { hours } \\
\text { lost per } \\
\text { year @ } \\
\mathbf{2 0 \%} \\
\text { DP of }\end{array}$ & $\begin{array}{l}\text { Value of } \\
\text { hours } \\
\text { lost per } \\
\text { year } \\
\text { @o\% } \\
\text { of DP }\end{array}$ \\
\hline 1 & 40 & 133450 & 150 & 890 & 5 & 4448 & 231313 & 23131 & 46263 & 69394 \\
\hline 2 & 40 & 175718 & 150 & 1171 & 5 & 5857 & 304578 & 30458 & 60916 & 91373 \\
\hline 3 & 40 & 240768 & 150 & 1605 & 5 & 8026 & 417331 & 41733 & 83466 & 125199 \\
\hline 4 & 40 & 311693 & 150 & 2078 & 5 & 10390 & 540268 & 54027 & 108054 & 162080 \\
\hline 5 & 40 & 489104 & 150 & 3261 & 5 & 16303 & 847780 & 84778 & 169556 & 254334 \\
\hline 6 & 40 & 632501 & 150 & 4217 & 5 & 21083 & 1096335 & 109634 & 219267 & 328901 \\
\hline 7 & 40 & 815855 & 150 & 5439 & 5 & 27195 & 1414149 & 141415 & 282830 & 424245 \\
\hline Total & & & & & & $\mathbf{9 3 3 0 3}$ & $\mathbf{4 8 5 1 7 5 4}$ & $\mathbf{4 8 5 1 7 5}$ & $\mathbf{9 7 0 3 5 1}$ & $\mathbf{1 4 5 5 5 2 6}$ \\
\hline
\end{tabular}

This example shows only 5 hours weekly lost

Appendix 12: Five hours weekly loss to absences by DPs per year 
Outputs from the sensitivity analysis of Normal routine hour loss and \% of doctors

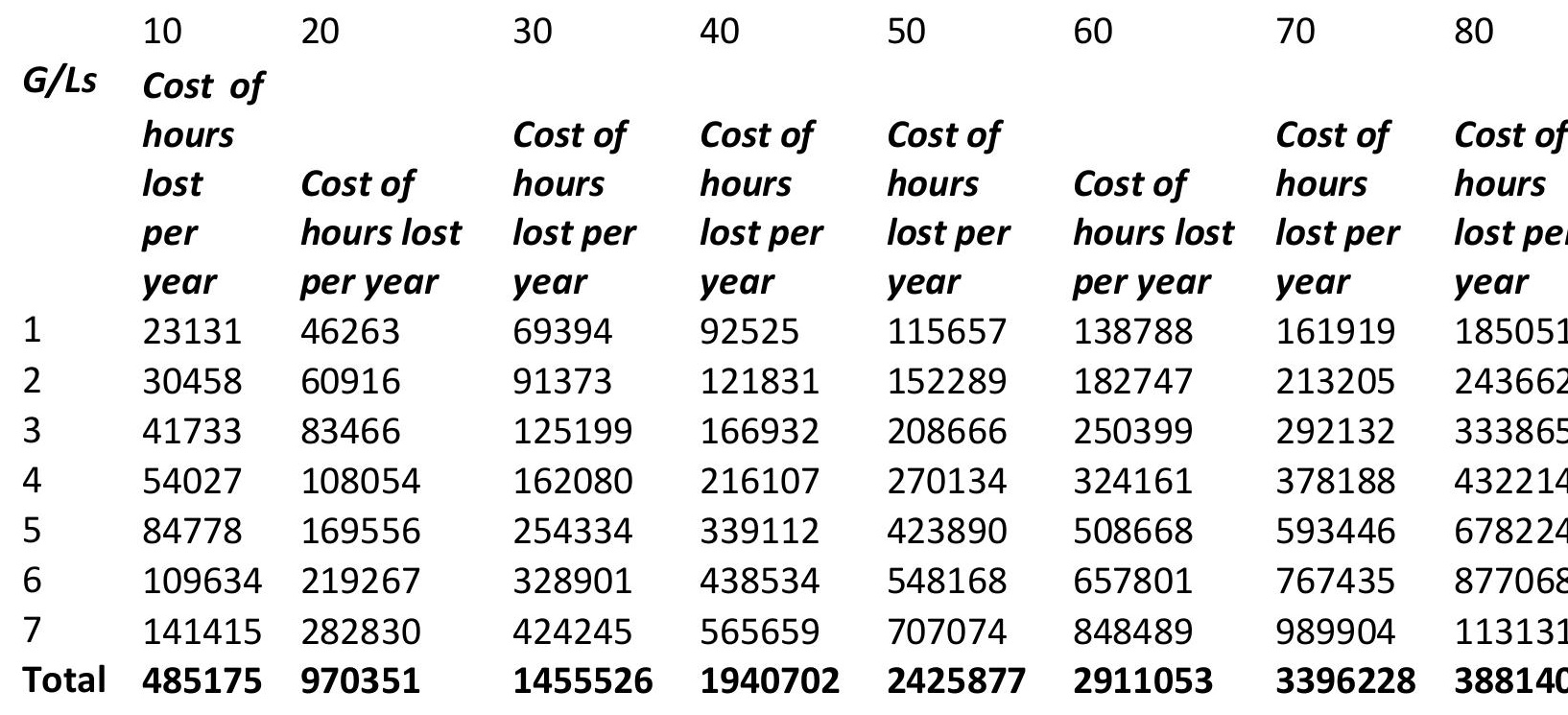


Appendix 13: Eight hours weekly loss to absences by DPs per ye

$\begin{array}{cccccccc}\text { G/Levels } & 10 & 20 & 30 & 40 & 50 & 60 & 70 \\ 1 & 37010 & 74020 & 111030 & 148041 & 185051 & 222061 & 259071 \\ 2 & 48732 & 97465 & 146197 & 194930 & 243662 & 292395 & 341127 \\ 3 & 66773 & 133546 & 200319 & 267092 & 333865 & 400638 & 467411 \\ 4 & 86443 & 172886 & 259329 & 345771 & 432214 & 518657 & 605100 \\ 5 & 135645 & 271290 & 406935 & 542579 & 678224 & 813869 & 949514 \\ 6 & 175414 & 350827 & 526241 & 701654 & 877068 & 1052482 & 1227895 \\ 7 & 226264 & 452528 & 678791 & 905055 & 1131319 & 1357583 & 1583847 \\ \text { Total } & \mathbf{7 7 6 2 8 1} & \mathbf{1 5 5 2 5 6 1} & \mathbf{2 3 2 8 8 4 2} & \mathbf{3 1 0 5 1 2 3} & \mathbf{3 8 8 1 4 0 3} & \mathbf{4 6 5 7 6 8 4} & \mathbf{5 4 3 3 9 6 5}\end{array}$

Appendix 14: 10 hours weekly loss to absences by DPs per year

$\begin{array}{lccccccc}\text { G/Levels } & 10 & 20 & 30 & 40 & 50 & 60 & 70 \\ 1 & 46263 & 92525 & 138788 & 185051 & 231313 & 277576 & 323839 \\ 2 & 60916 & 121831 & 182747 & 243662 & 304578 & 365493 & 426409 \\ 3 & 83466 & 166932 & 250399 & 333865 & 417331 & 500797 & 584264 \\ 4 & 108054 & 216107 & 324161 & 432214 & 540268 & 648321 & 756375 \\ 5 & 169556 & 339112 & 508668 & 678224 & 847780 & 1017336 & 1186892 \\ 6 & 219267 & 438534 & 657801 & 877068 & 1096335 & 1315602 & 1534869 \\ 7 & 282830 & 565659 & 848489 & 1131319 & 1414149 & 1696978 & 1979808 \\ \text { Total } & \mathbf{9 7 0 3 5 1} & \mathbf{1 9 4 0 7 0 2} & \mathbf{2 9 1 1 0 5 3} & \mathbf{3 8 8 1 4 0 3} & \mathbf{4 8 5 1 7 5 4} & \mathbf{5 8 2 2 1 0 5} & \mathbf{6 7 9 2 4 5}\end{array}$


Appendix 15: 12 hour's weekly loss to absences by DPs per year

$\begin{array}{ccccccccr}\text { G/Levels } & 10 & 20 & 30 & 40 & 50 & 60 & 70 & 8 \\ 1 & 55515 & 111030 & 166546 & 222061 & 277576 & 333091 & 388606 & 444 \\ 2 & 73099 & 146197 & 219296 & 292395 & 365493 & 438592 & 511691 & 584 \\ 3 & 100159 & 200319 & 300478 & 400638 & 500797 & 600957 & 701116 & 801 \\ 4 & 129664 & 259329 & 388993 & 518657 & 648321 & 777986 & 907650 & 103 \\ 5 & 203467 & 406935 & 610402 & 813869 & 1017336 & 1220804 & 1424271 & 162 \\ 6 & 263120 & 526241 & 789361 & 1052482 & 1315602 & 1578722 & 1424271 & 210 \\ 7 & 339396 & 678791 & 1018187 & 1357583 & 1696978 & 2036374 & 2375770 & 271 ! \\ \text { Total } & \mathbf{1 1 6 4 4 2 1} & \mathbf{2 3 2 8 8 4 2} & \mathbf{3 4 9 3 2 6 3} & \mathbf{4 6 5 7 6 8 4} & \mathbf{5 8 2 2 1 0 5} & \mathbf{6 9 8 6 5 2 6} & \mathbf{7 7 3 3 3 7 5} & \mathbf{9 3 1 !}\end{array}$

Appendix 16: 15 hours weekly loss to absences by DPs per year

G/Levels

$\begin{array}{lcccccccc} & 10 & 20 & 30 & 40 & 50 & 60 & 70 & 80 \\ 1 & 69394 & 138788 & 208182 & 277576 & 346970 & 416364 & 485758 & 555152 \\ 2 & 91373 & 182747 & 274120 & 365493 & 456867 & 548240 & 639614 & 730986.9 \\ 3 & 125199 & 250399 & 375598 & 500797 & 625997 & 751196 & 876396 & 1001595 \\ 4 & 162080 & 324161 & 486241 & 648321 & 810402 & 972482 & 1134563 & 1296643 \\ 5 & 203467 & 508668 & 763002 & 1017336 & 1271670 & 1526004 & 1780339 & 2034673 \\ 6 & 328901 & 657801 & 986702 & 1315602 & 1644503 & 1973403 & 2302304 & 2631204 \\ 7 & 424245 & 848489 & 1272734 & 1696978 & 2121223 & 2545468 & 2969712 & 3393957 \\ \text { Total } & \mathbf{1 4 0 4 6 5 9} & \mathbf{2 9 1 1 0 5 3} & \mathbf{4 3 6 6 5 7 9} & \mathbf{5 8 2 2 1 0 5} & \mathbf{7 2 7 7 6 3 1} & \mathbf{8 7 3 3 1 5 8} & \mathbf{1 0 1 8 8 6 8 4} & \mathbf{1 1 6 4 4 2 1}\end{array}$


Appendix 17: 20 hour's weekly loss to absences by DPs per year

$\begin{array}{ccccccccc} & 10 & 20 & 30 & 40 & 50 & 60 & 70 & 80 \\ 1 & 92525 & 185051 & 277576 & 370101 & 462627 & 555152 & 647677 & 740203 \\ 2 & 121831 & 243662 & 365493 & 487325 & 609156 & 730987 & 852818 & 974649 \\ 3 & 166932 & 333865 & 500797 & 667730 & 834662 & 1001595 & 1168527 & 1335460 \\ 4 & 216107 & 432214 & 648321 & 864429 & 1080536 & 1296643 & 1512750 & 1728857 \\ 5 & 339112 & 678224 & 648321 & 1356448 & 1695561 & 2034673 & 2373785 & 2712897 \\ 6 & 438534 & 877068 & 1315602 & 1754136 & 2192670 & 2034673 & 3069738 & 3508272 \\ 7 & 565659 & 1131319 & 1696978 & 2262638 & 2828297 & 3393957 & 3959616 & 4525276 \\ \text { Total } & \mathbf{1 9 4 0 7 0 2} & \mathbf{3 8 8 1 4 0 3} & \mathbf{5 4 5 3 0 9 0} & \mathbf{7 7 6 2 8 0 7} & \mathbf{9 7 0 3 5 0 9} & \mathbf{1 1 0 4 7 6 7 9} & \mathbf{1 3 5 8 4 9 1 2} & \mathbf{1 5 5 2 5 6 1 4}\end{array}$


Appendix 18: Consolidated Medical Salary Structure (on-call hour allowance fo

\begin{tabular}{|c|c|c|c|c|c|c|c|c|c|c|c|}
\hline Grade/Level & & & $\begin{array}{r}\text { Consolidated } \\
2\end{array}$ & $\begin{array}{l}\text { Medical salary } \\
3\end{array}$ & y structure (CO & DNMESS) & $\begin{array}{r}\text { CALL HOUR A } \\
6\end{array}$ & TLOWANCE 7 & 8 & 9 & 10 \\
\hline & Consololidat & 109707.33 & $\overline{0}$ & 0 & 0 & 0 & 0 & 0 & 0 & 0 & \\
\hline & $\begin{array}{l}\text { Hazard } \\
\text { Call Duty }\end{array}$ & $\begin{array}{r}5000 \\
60120\end{array}$ & & & & & & & & & \\
\hline & Teacl & 9371 & & & & & & & & & \\
\hline & & 193570.33 & & & & & & & & & \\
\hline 2 & $\begin{array}{l}\text { Consololidat } \\
\text { Hazard } \\
\text { Call Duty } \\
\text { Teaching All c } \\
\text { Rural Posting }\end{array}$ & $\begin{array}{r}130216 \\
5000 \\
67160 \\
11246 \\
11246 \\
\mathbf{2 2 4 8 6 7 . 5 2}\end{array}$ & $\begin{array}{r}133410 \\
5000 \\
70120 \\
11490 \\
11490 \\
\mathbf{2 3 1 5 0 9 . 7 5}\end{array}$ & $\begin{array}{r}136604 \\
500 \\
73080 \\
11735 \\
11735 \\
\mathbf{2 3 3 6 5 3 . 9 8}\end{array}$ & $\begin{array}{r}139798 \\
5000 \\
76040 \\
11980 \\
11980 \\
\mathbf{2 4 4 7 9 8 . 2 1}\end{array}$ & $\begin{array}{r}142992 \\
5000 \\
79000 \\
12225 \\
12225 \\
\mathbf{2 5 1 4 4 2 . 4 4}\end{array}$ & $\begin{array}{r}146187 \\
5000 \\
81960 \\
12470 \\
12470 \\
\mathbf{2 5 8 0 8 6 . 6 7}\end{array}$ & $\begin{array}{r}149381 \\
5000 \\
84920 \\
12715 \\
12715 \\
\mathbf{2 6 4 7 3 0 . 9}\end{array}$ & $\begin{array}{r}152575 \\
5000 \\
87920 \\
12960 \\
12960 \\
\mathbf{2 7 1 4 1 5 . 1 3}\end{array}$ & $\begin{array}{r}155769 \\
5000 \\
90880 \\
13205 \\
13205 \\
\mathbf{2 7 8 0 5 9 . 3 5}\end{array}$ & $\begin{array}{r}15896 \\
500 \\
93840 \\
13450 \\
13450 \\
\mathbf{2 8 4 7 0 3 . 5}\end{array}$ \\
\hline 3 & $\begin{array}{l}\text { Consololidat } \\
\text { Hazard } \\
\text { Call Duty } \\
\text { Teaching Allc } \\
\text { Rural Posting }\end{array}$ & $\begin{array}{r}185636 \\
5000 \\
95320 \\
13495 \\
13495 \\
\mathbf{3 1 2 9 4 5 . 6 7}\end{array}$ & $\begin{array}{r}189810 \\
5000 \\
98440 \\
13764 \\
13764 \\
\mathbf{3 2 0 7 7 8 . 4 5}\end{array}$ & $\begin{array}{r}193985 \\
5000 \\
101560 \\
14033 \\
14033 \\
\mathbf{3 2 8 6 1 1 . 2 5}\end{array}$ & $\begin{array}{r}198160 \\
5000 \\
104680 \\
11980 \\
11980 \\
\mathbf{3 3 1 8 0 0 . 0 4}\end{array}$ & $\begin{array}{r}202335 \\
5000 \\
107800 \\
14571 \\
14571 \\
\mathbf{3 4 4 2 7 6 . 8 4}\end{array}$ & $\begin{array}{r}206510 \\
5000 \\
110920 \\
14840 \\
14840 \\
\mathbf{3 5 2 1 0 9 . 6 3}\end{array}$ & $\begin{array}{r}210684 \\
5000 \\
114040 \\
15109 \\
15109 \\
\mathbf{3 5 9 9 4 2 . 4 2}\end{array}$ & $\begin{array}{r}214859 \\
5000 \\
117160 \\
15378 \\
15378 \\
\mathbf{3 6 7 7 7 5 . 2 1}\end{array}$ & $\begin{array}{r}219034 \\
5000 \\
120280 \\
15647 \\
15647 \\
\mathbf{3 7 5 6 0 8 . 0 1}\end{array}$ & $\begin{array}{r}223200 \\
5000 \\
123400 \\
15917 \\
15917 \\
\mathbf{3 8 3 4 4 2 . 8}\end{array}$ \\
\hline 4 & $\begin{array}{l}\text { Consololidat } \\
\text { Hazard } \\
\text { Call Duty } \\
\text { Teaching All c } \\
\text { Rural Posting }\end{array}$ & $\begin{array}{r}238791 \\
5000 \\
124960 \\
16194 \\
16194 \\
\mathbf{4 0 1 1 3 9 . 0 2}\end{array}$ & $\begin{array}{r}245094 \\
5000 \\
128320 \\
16594 \\
16594 \\
\mathbf{4 1 1 6 0 1 . 7 7}\end{array}$ & $\begin{array}{r}251397 \\
5000 \\
131680 \\
16994 \\
16994 \\
\mathbf{4 2 2 0 6 4 . 5 2}\end{array}$ & $\begin{array}{r}257699 \\
5000 \\
135040 \\
17394 \\
17394 \\
\mathbf{4 3 2 5 2 7 . 2 7}\end{array}$ & $\begin{array}{r}264002 \\
5000 \\
138400 \\
17794 \\
17794 \\
\mathbf{4 4 2 9 9 0 . 0 2}\end{array}$ & $\begin{array}{r}270305 \\
5000 \\
141760 \\
18194 \\
18194 \\
\mathbf{4 5 3 4 5 2 . 7 7}\end{array}$ & $\begin{array}{r}276608 \\
5000 \\
145120 \\
18594 \\
18594 \\
\mathbf{4 6 3 9 1 5 . 5 2}\end{array}$ & $\begin{array}{r}282910 \\
5000 \\
148480 \\
18994 \\
18994 \\
\mathbf{4 7 4 3 7 8 . 2 7}\end{array}$ & $\begin{array}{r}289213 \\
5000 \\
151840 \\
19394 \\
19394 \\
\mathbf{4 8 4 8 4 1 . 0 2}\end{array}$ & $\begin{array}{r}29551 \\
5000 \\
155200 \\
1979 \\
1979 \\
\mathbf{4 9 5 3 0 3 . 7}\end{array}$ \\
\hline 5 & $\begin{array}{l}\text { Consololidat } \\
\text { Hazard } \\
\text { Call Duty } \\
\text { Teaching Allc } \\
\text { Rural Posting } \\
\text { Clinical Duty } \\
\text { Specialist All }\end{array}$ & \begin{tabular}{|r|}
301841 \\
5000 \\
156880 \\
20242 \\
20242 \\
25285 \\
91089 \\
$\mathbf{6 2 0 5 7 9 . 1 4}$ \\
\end{tabular} & $\begin{array}{r}310835 \\
5000 \\
161400 \\
20792 \\
24542 \\
26528 \\
93564 \\
\mathbf{6 4 2 6 6 0 . 5 5} \\
\end{array}$ & $\begin{array}{r}319828 \\
5000 \\
165920 \\
21342 \\
21342 \\
27770 \\
96039 \\
657240.95 \\
\end{array}$ & \begin{tabular}{|r|}
328821 \\
5000 \\
170440 \\
21892 \\
21892 \\
29012 \\
98514 \\
$\mathbf{6 7 5 5 7 1 . 3 6}$ \\
\end{tabular} & $\begin{array}{r}337815 \\
5000 \\
174960 \\
22442 \\
22442 \\
30254 \\
100989 \\
\mathbf{6 9 3 9 0 1 . 7 7}\end{array}$ & $\begin{array}{r}346808 \\
5000 \\
179480 \\
22992 \\
22992 \\
31496 \\
103464 \\
\mathbf{7 1 2 2 3 2 . 1 8}\end{array}$ & \begin{tabular}{|r|}
355802 \\
5000 \\
184000 \\
23542 \\
23542 \\
32738 \\
105939 \\
$\mathbf{7 3 0 5 6 2 . 5 9}$ \\
\end{tabular} & $\begin{array}{r}364795 \\
5000 \\
188520 \\
24092 \\
24092 \\
33980 \\
108414 \\
\mathbf{7 4 8 8 9 3}\end{array}$ & $\begin{array}{r}373788 \\
5000 \\
193040 \\
24642 \\
24642 \\
35222 \\
110889 \\
767223.45 \\
\end{array}$ & 174960 \\
\hline 6 & $\begin{array}{l}\text { Consololidat } \\
\text { Hazard } \\
\text { Call Duty } \\
\text { Teaching Allc } \\
\text { Rural Postins } \\
\text { Clinical Duty } \\
\text { Specialist All }\end{array}$ & $\begin{array}{r}400192 \\
5000 \\
190800 \\
25303 \\
25303 \\
27967 \\
113861 \\
\mathbf{7 8 8 4 2 6 . 1}\end{array}$ & $\begin{array}{r}411678 \\
5000 \\
196240 \\
25953 \\
25953 \\
29460 \\
116786 \\
\mathbf{8 1 1 0 6 9 . 6 8}\end{array}$ & $\begin{array}{r}423163 \\
5000 \\
201680 \\
26603 \\
26603 \\
30952 \\
119711 \\
\mathbf{8 3 3 7 1 2 . 2 6} \\
\end{array}$ & $\begin{array}{r}434649 \\
5000 \\
207120 \\
27253 \\
27253 \\
32445 \\
122636 \\
\mathbf{8 5 6 3 5 5 . 8 3}\end{array}$ & \begin{tabular}{r|}
446134 \\
5000 \\
212560 \\
27903 \\
27903 \\
33938 \\
125561 \\
$\mathbf{8 7 8 9 9 9 . 4 1}$
\end{tabular} & $\begin{array}{r}457620 \\
5000 \\
218000 \\
28553 \\
28553 \\
35430 \\
128486 \\
\mathbf{9 0 1 6 4 1 . 9 9}\end{array}$ & $\begin{array}{r}469106 \\
5000 \\
223440 \\
29203 \\
29203 \\
36923 \\
131411 \\
\mathbf{9 2 4 2 8 5 . 5 7}\end{array}$ & $\begin{array}{r}480591 \\
5000 \\
228880 \\
29853 \\
29853 \\
38416 \\
134336 \\
\mathbf{9 4 6 9 2 9 . 1 4}\end{array}$ & $\begin{array}{r}492077 \\
5000 \\
234320 \\
30503 \\
30503 \\
39908 \\
137261 \\
\mathbf{9 6 9 5 7 1 . 7 1} \\
\end{array}$ & 212560 \\
\hline 7 & $\begin{array}{l}\text { Consololidat } \\
\text { Hazard }\end{array}$ & $\begin{array}{r}529168 \\
5000\end{array}$ & $\begin{array}{r}543319 \\
5000\end{array}$ & $\begin{array}{r}557470 \\
5000\end{array}$ & $\begin{array}{r}571621 \\
5000\end{array}$ & $\begin{array}{r}585772 \\
5000\end{array}$ & $\begin{array}{r}599922 \\
5000\end{array}$ & $\begin{array}{r}614073 \\
5000\end{array}$ & $\begin{array}{r}628224 \\
5000\end{array}$ & $\begin{array}{r}642375 \\
5000\end{array}$ & \\
\hline
\end{tabular}


Appendix 19: Five hours weekly loss to absences by DPs on on-call hou

Outputs from the computation of On-call hour loss and \% of doctors engage

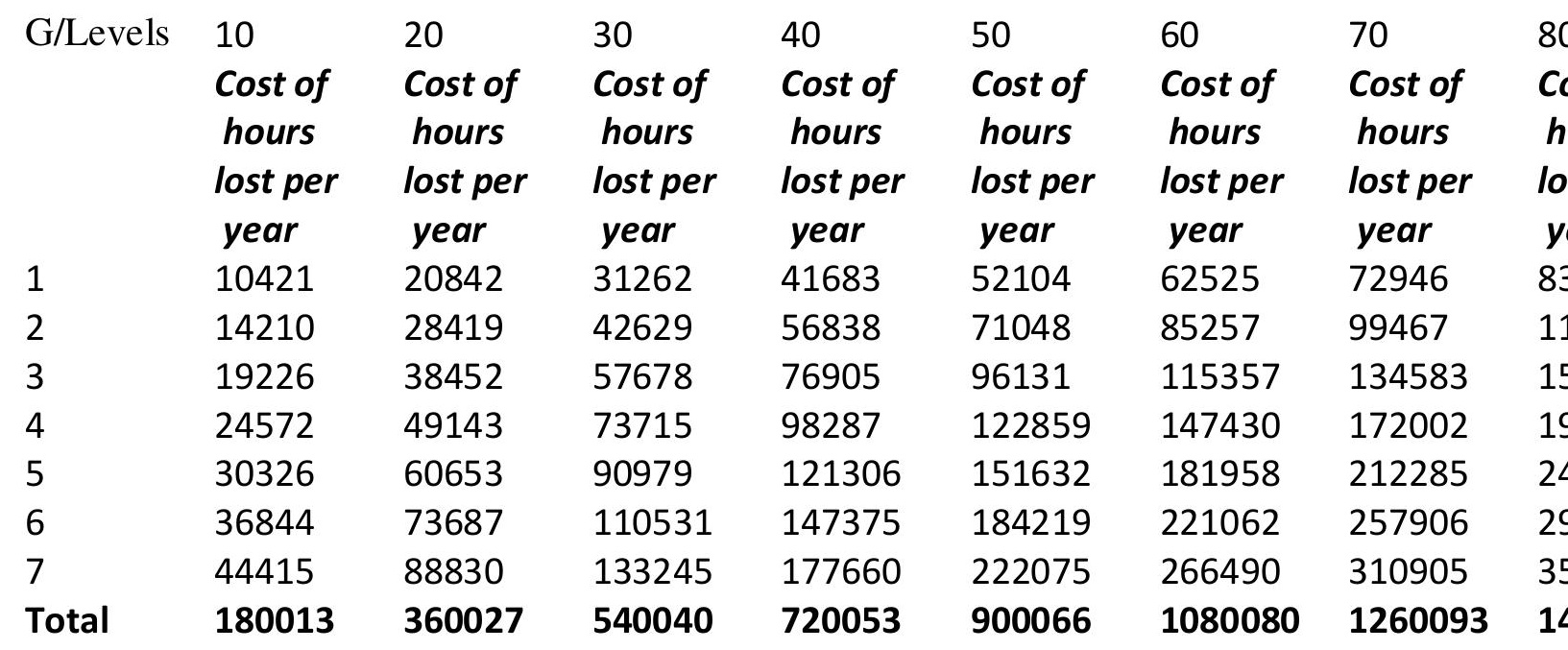


Appendix 20: Six hours weekly loss to absences by DPs on on-call hour per

$\begin{array}{cccccccccc}\text { G/Levels } & 10 & 20 & 30 & 40 & 50 & 60 & 70 & 80 & \\ 1 & 12505 & 25010 & 37515 & 50020 & 62525 & 75030 & 87535 & 100040 & 11 \\ 2 & 17051 & 34103 & 51154 & 68206 & 85257 & 102309 & 119360 & 136412 & 15 \\ 3 & 23071 & 46143 & 69214 & 92285 & 115357 & 138428 & 161500 & 184571 & 20 \\ 4 & 29486 & 58972 & 88458 & 117944 & 147430 & 176916 & 206403 & 235889 & 26 \\ 5 & 36392 & 72783 & 109175 & 145567 & 181958 & 218350 & 254742 & 291133 & 32 \\ 6 & 44212 & 88425 & 132637 & 176850 & 221062 & 265275 & 309487 & 353700 & 39 \\ 7 & 53298 & 106596 & 159894 & 213192 & 266490 & 319788 & 373085 & 426383 & 47 \\ \text { Total } & \mathbf{2 1 6 0 1 6} & \mathbf{4 3 2 0 3 2} & \mathbf{6 4 8 0 4 8} & \mathbf{8 6 4 0 6 4} & \mathbf{1 0 8 0 0 8 0} & \mathbf{1 2 9 6 0 9 6} & \mathbf{1 5 1 2 1 1 2} & \mathbf{1 7 2 8 1 2 8} & \mathbf{1 9 6}\end{array}$

Appendix 21: Seven hours weekly loss to absences by DPs on on-call hour pe

G/Levels

$\begin{array}{cccccccccr} & 10 & 20 & 30 & 40 & 50 & 60 & 70 & 80 & 9 \\ 1 & 14589 & 29178 & 43767 & 58356 & 72946 & 87535 & 102124 & 116713 & 131 \\ 2 & 19893 & 39787 & 59680 & 79573 & 99467 & 119360 & 139254 & 159147 & 179 \\ 3 & 26917 & 53833 & 80750 & 107666 & 134583 & 161500 & 188416 & 215333 & 242 \\ 4 & 34400 & 68801 & 103201 & 137602 & 172002 & 206403 & 240803 & 275203 & 309 \\ 5 & 42457 & 84914 & 127371 & 169828 & 212285 & 254742 & 297199 & 339656 & 382 \\ 6 & 51581 & 103162 & 154744 & 206325 & 257906 & 309487 & 361069 & 412650 & 464 \\ 7 & 62181 & 124362 & 186543 & 248724 & 310905 & 373085 & 435266 & 497447 & 559 \\ \text { Total } & \mathbf{2 5 2 0 1 9} & \mathbf{5 0 4 0 3 7} & \mathbf{7 5 6 0 5 6} & \mathbf{1 0 0 8 0 7 4} & \mathbf{1 2 6 0 0 9 3} & \mathbf{1 5 1 2 1 1 2} & \mathbf{1 7 6 4 1 3 0} & \mathbf{2 0 1 6 1 4 9} & \mathbf{2 2 6 8}\end{array}$


Appendix 22: Eight hours weekly loss to absences by DPs on on-call hour pe

$\begin{array}{ccccccccc}\mathrm{G} / \mathrm{L} & 10 & 20 & 30 & 40 & 50 & 60 & 70 & 80 \\ 1 & 16673 & 33347 & 50020 & 66693 & 83366 & 100040 & 116713 & 133386 \\ 2 & 22735 & 45471 & 68206 & 90941 & 113676 & 136412 & 159147 & 181882 \\ 3 & 30762 & 61524 & 92285 & 123047 & 153809 & 184571 & 215333 & 246095 \\ 4 & 39315 & 78630 & 117944 & 157259 & 196574 & 235889 & 275203 & 314518 \\ 5 & 39315 & 97044 & 145567 & 194089 & 242611 & 291133 & 339656 & 388178 \\ 6 & 58950 & 117900 & 176850 & 235800 & 294750 & 353700 & 412650 & 471600 \\ 7 & 71064 & 142128 & 213192 & 284256 & 355319 & 426383 & 497447 & 568511 \\ \text { Total } & \mathbf{2 7 8 8 1 4} & \mathbf{5 7 6 0 4 3} & \mathbf{8 6 4 0 6 4} & \mathbf{1 1 5 2 0 8 5} & \mathbf{1 4 4 0 1 0 6} & \mathbf{1 7 2 8 1 2 8} & \mathbf{2 0 1 6 1 4 9} & \mathbf{2 3 0 4 1 7 0}\end{array}$


Appendix 23: Nine hours weekly loss to absences by DPs on on-call hour per

$\begin{array}{ccccccccr}\mathrm{G} / \mathrm{L} & 10 & 20 & 30 & 40 & 50 & 60 & 70 & 80 \\ 1 & 18757 & 37514.88 & 56272.32 & 75029.76 & 93787.2 & 112544.6 & 131302.08 & 15005 \\ 2 & 25577 & 51154.39 & 76731.58 & 102308.8 & 127886 & 153463.2 & 179040.35 & 20461 \\ 3 & 34607 & 69214.08 & 103821.1 & 138428.2 & 173035.2 & 207642.2 & 242249.28 & 27685 \\ 4 & 44229 & 88458.24 & 132687.4 & 218350.1 & 221145.6 & 265374.7 & 309603.84 & 3538 \\ 5 & 54588 & 109175 & 163762.6 & 218350.1 & 272937.6 & 327525.1 & 382112.64 & 43670 \\ 6 & 66319 & 132637.4 & 198956.2 & 265274.9 & 331593.6 & 397912.3 & 309603.84 & 53054 \\ 7 & 79947 & 159893.8 & 239840.6 & 319787.5 & 399734.4 & 479681.3 & 559628.16 & 6395 \\ \text { Total } & \mathbf{3 2 4 0 2 4} & \mathbf{6 4 8 0 4 7 . 8} & \mathbf{9 7 2 0 7 1 . 7} & \mathbf{1 3 3 7 5 2 9} & \mathbf{1 6 2 0 1 2 0} & \mathbf{1 9 4 4 1 4 3} & \mathbf{2 1 1 3 5 4 0 . 2} & \mathbf{2 5 9 2 1}\end{array}$


Appendix 24: 10 hours weekly loss to absences by DPs on on-call hours per y

\begin{tabular}{|c|c|c|c|c|c|c|c|}
\hline $\mathrm{G} / \mathrm{L}$ & 10 & 20 & 30 & 40 & 50 & 60 & 70 \\
\hline 1 & 20841.6 & 41683.2 & 62524.8 & 83366.4 & 104208 & 125049.6 & 145891.2 \\
\hline 2 & 28419.1 & 56838.21 & 85257.31 & 113676.4 & 142095.5 & 170514.6 & 198933.72 \\
\hline 3 & 38452.27 & 76904.53 & 147430.4 & 153809.1 & 192261.3 & 230713.6 & 269165.87 \\
\hline 4 & 49143.47 & 98286.93 & 147430.4 & 196573.9 & 245717.3 & 294860.8 & 344004.27 \\
\hline 5 & 60652.8 & 121305.6 & 181958.4 & 242611.2 & 303264 & 363916.8 & 424569.6 \\
\hline 6 & 73687.47 & 147374.9 & 221062.4 & 294749.9 & 368437.3 & 442124.8 & 515812.27 \\
\hline 7 & 88829.87 & 177659.7 & 266489.6 & 355319.5 & 444149.3 & 532979.2 & 621809.07 \\
\hline Total & 360026.6 & 720053.1 & 1112153 & 1440106 & 1800133 & 2160159 & 2520186 \\
\hline
\end{tabular}


Appendix 25: Ethics from UNTH, Ituzu-Ozalla

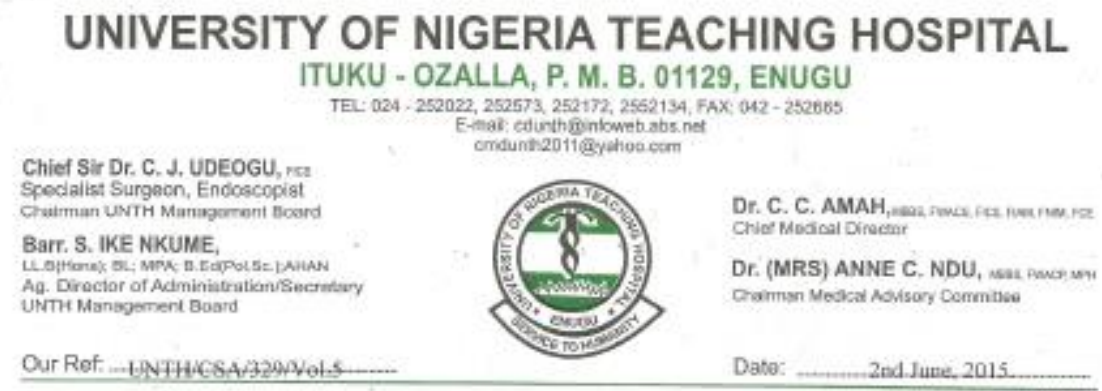

NHREC/05/O1/2008B - FWA00002458 - IREO0002323

EIHICAL CIFADANCF CFDTIFICATE

TOPIO: DUAL PRACTICE OF MEDICAL PROFESSIONALS IN

PUBLIC HEALTH FACILITIES IN NIGERIA:

AN ECONOMIC AND POLICY ANALYSIS

sY:

BARTHOLOMEW S. EZE

rOR:

A Ph.D THESIS OF THE COLLEGE OF HEALTH AND HUMAN SCIENCES SWANSEA UNIVERSITY, UNITED KINGDOM

This reseanch perject on the above bopic was reviewed nnd approved by the Liniversity of Nigeria Hezlth Research Ethics Conmitioe. This certiflese is valid for oue year from date of tssue. 


\section{ENUGU STATE MINISTRY OF HEALTH ETHICAL COMMITTEE ON RESEARCH PROJECTS}

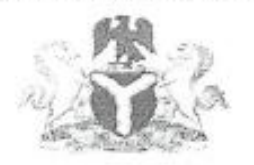

Ref. No.: $M H / M S D / E C / 0181$

Date: $22^{\text {nd }}$ June 2015.

The Researcher,

Bartholomew S. Eze,

College of Health and Human Sciences,

Swansea University,

United Kingdom.

$\underline{\text { Re-Dual practice of medical professionals in Nigeria: An economic and policy }}$

I refer to your request for permission to carry out a study / research on the above health issue and to inform you that approval has been granted to you.

Ethical Guideline

1. You are to keep to the principles of informed consent by obtaining a signed/ thumb printed informed consent of subjects, parents/ legally accepted representative.

2. You are to deposit two (2) copies of the result of your study to the ethical committee of the State Ministry of Health.

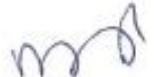

Dr. Ejeh M.N.

Chairman, Ethical Committee on Research 
Appendix 27: Ethics from the College of Human and Health Sciences

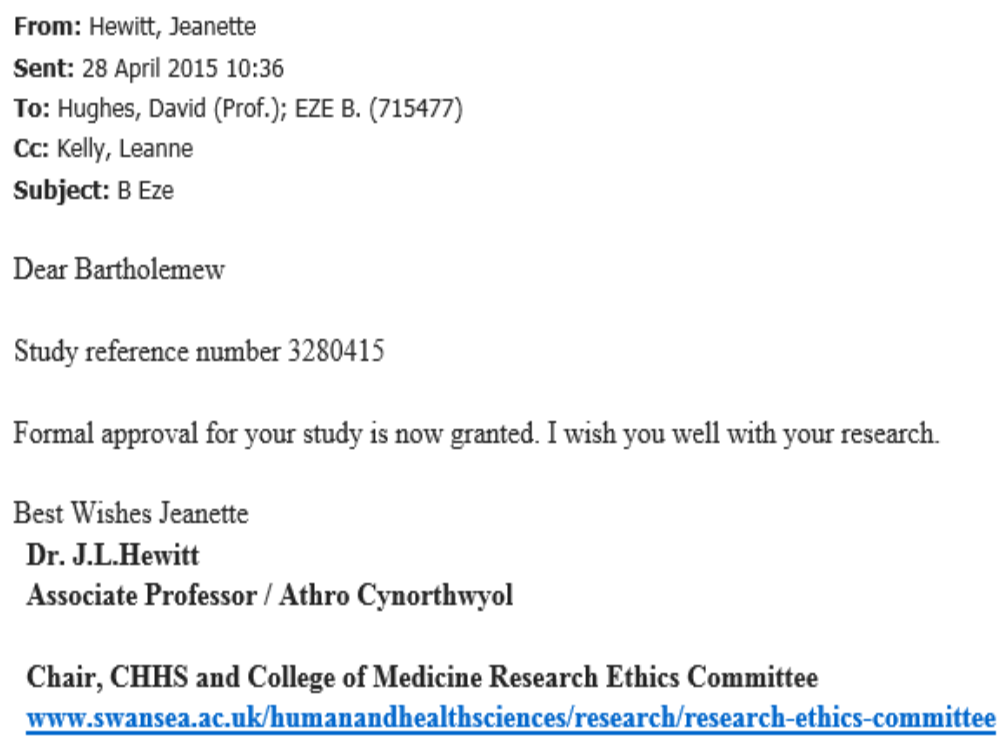

Department of Philosophy, History \& Law / Uwch Ddarlithydd, Adran Athroniaeth, Hanes a'r Gyfraith College of Human and Health Sciences / Coleg y Gwyddorau Dynol ac Iechyd Swansea University / Prifysgol Abertawe

Singleton Park / Parc Singleton

Swansea / Abertawe

Wales / Cymruc

SA2 SPP

www.swansea.ac.uk | www.abertawe.ac.uk 
Appendix 28: Participant consent form

\section{Participant consent form}

\section{Title of project}

Dual practice of medical professionals in public hospitals in Nigeria: An economic and policy analysis

\section{Please tick box}

- I confirm that I have read and understand the information sheet for the above study and have had the opportunity to ask questions.

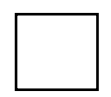

- I confirm that the investigator has explained the aim of the study to the focus group participants

- I understand that my participation is voluntary and that I am free to withdraw from the project at any time, up to the point of completion, without having to give a reason and without any consequences. If I exercise my right to withdraw and I don't want my data to be used, any data which have been collected from me will be destroyed.

- I understand that any information recorded in the investigation will remain confidential and no information that identifies me will be made

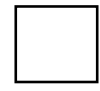
publicly available.

- I consent to be a participant in the project

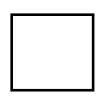

Name of participant

Date

Signature

Bartholomew S. Eze

Name of investigator

Date

Signature 
Appendix 29: An overview of some of the main studies reviewed

\begin{tabular}{|c|c|c|c|c|c|}
\hline $\mathbf{S} / \mathbf{N}$ & Author/date & Aim of study/paper & $\begin{array}{l}\text { Type of study } \\
\text { information }\end{array}$ & Main findings/conclusions & $\begin{array}{l}\text { Strengths } \\
\text { limitations }\end{array}$ \\
\hline 1. & $\begin{array}{l}\text { Morris et al. } \\
2008 \\
J R \text { Soc Med, } \\
\text { Analysis of } \\
\text { consultants NHS } \\
\text { and private } \\
\text { income in } \\
\text { England in } 2003 \text { ' }\end{array}$ & $\begin{array}{lr}\text { The } & \text { paper } \\
\text { investigates the } \\
\text { magnitude } \\
\text { determinants and } \\
\text { consultants, NHS } \\
\text { and private income }\end{array}$ & $\begin{array}{l}\text { Secondary data } \\
\text { analysis } \\
\text { (Quantitative) }\end{array}$ & $\begin{array}{l}\text {-The } 10 \% \text { rule was } \\
\text { exceeded by the full-time } \\
\text { NHS consultants } \\
\text {-The younger consultants } \\
\text { earned higher private } \\
\text { income than other older } \\
\text { age group } \\
\text {-Conditional mean income } \\
\text { was highest among certain } \\
\text { specialty (plastic surgery) } \\
\text {-Total NHS and private } \\
\text { income vary by age, } \\
\text { specialty and Health } \\
\text { Authority }\end{array}$ & $\begin{array}{l}\text { - the self- } \\
\text { income upo } \\
\text { the analys } \\
\text { based ca } \\
\text { misleading } \\
\text {-cos the } \\
\text { income cou } \\
\text { come fron } \\
\text { sources ap } \\
\text { private clinic }\end{array}$ \\
\hline 2 & 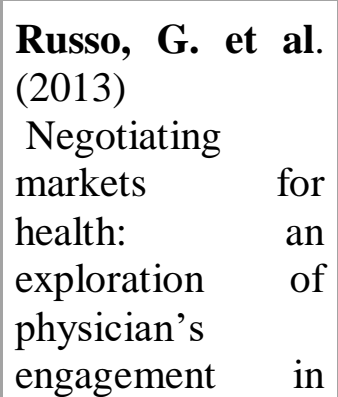 & $\begin{array}{l}\text { The paper looks at } \\
\text { the dual practice } \\
\text { pattern in three } \\
\text { African cities to } \\
\text { investigate the } \\
\text { influence of local } \\
\text { determinants on } \\
\text { private practice }\end{array}$ & $\begin{array}{l}\text { Questionnaire } \\
\text { and qualitative } \\
\text { interview }\end{array}$ & $\begin{array}{l}\text {-many types of DP exist } \\
\text {-Prevalence of dual } \\
\text { practice in the three cities } \\
\text {-Regulating and (Health } \\
\text { systems governance) } \\
\text { implementing institution } \\
\text { was strong in Cape Verde } \\
\text {-weak in Guinea Bissau }\end{array}$ & $\begin{array}{l}\text {-though an } \\
\text { paper tha } \\
\text { beyond mode } \\
\text { to collect } \\
\text { primary data } \\
\text { But failed to } \\
\text { detail how } \\
\text { contributed }\end{array}$ \\
\hline
\end{tabular}




\begin{tabular}{|c|c|c|c|c|c|}
\hline & $\begin{array}{l}\text { dual practice in } \\
\text { three African } \\
\text { capital cities }\end{array}$ & & & $\begin{array}{l}\text {-mixed strength in } \\
\text { Mozambique } \\
\text {-market structure shows } \\
\text { that Cape Verde are paid } \\
\text { higher (Russo, et al., } \\
2013 \text { b) ranging from USD } \\
903 \text { and } 1802 \text { with pension } \\
\text { benefits } \\
\text { While remunerative } \\
\text { exclusivity contract awaits } \\
\text { those in key political post } \\
\text { who would forgo DP } \\
\text {-In Guinea B. the pay for } \\
\text { physicians are very low } \\
\text { (315 and } 344 \text { USD with } \\
\text { limited allowances }\end{array}$ & $\begin{array}{l}\text { health syster } \\
\text { study areas } \\
\text { did it sh } \\
\text { economic ir } \\
\text { the practice. } \\
\text { - }\end{array}$ \\
\hline 3 & $\begin{array}{l}\text { Paul Ferrinho et } \\
\text { al. (1998). How } \\
\text { and why public } \\
\text { sector doctors } \\
\text { engage in private } \\
\text { practice } \\
\text { HP and Planning }\end{array}$ & $\begin{array}{l}\text {-investigate types } \\
\text { side-line activities } \\
\text { undertaken by DPs, } \\
\text { motivation for DP } \\
\text { and reasons why } \\
\text { public doctors in } \\
\text { PSAC have not left } \\
\text { the public sector }\end{array}$ & $\begin{array}{l}\text { Questionnaire } \\
\text { study }\end{array}$ & $\begin{array}{l}\text {-income generating } \\
\text { activities include - extra } \\
\text { income from non-medical } \\
\text { sources } \\
\text {-extra income from direct } \\
\text { professional activity } \\
\text {-support by the family } \\
\text { (how?) } \\
\text { - median equivalent of } \\
\text { one-month public sector } \\
\text { salary i.e. equivalent to just }\end{array}$ & $\begin{array}{l}\text { method wo } \\
\text { concentrated } \\
\text { the public } \\
\text { which carry } \\
\text { matters- the } \\
\text { of those ha } \\
\text { effect on } \\
\text { attending } \\
\text { hospitals } \\
\text { - that is the } 1 \\
\text { where DP ac }\end{array}$ \\
\hline
\end{tabular}




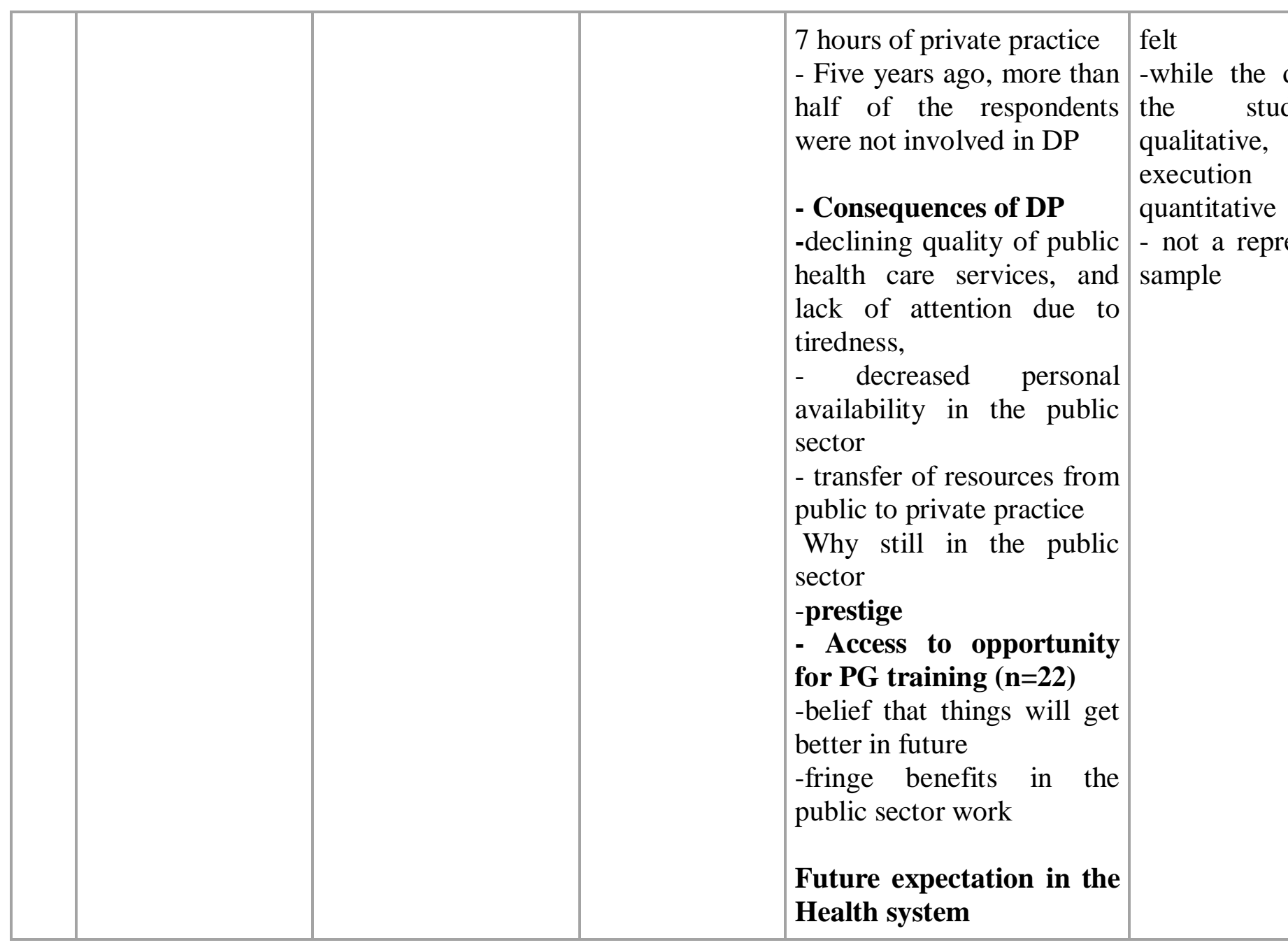




\begin{tabular}{|c|c|c|c|c|c|}
\hline & & & & $\begin{array}{l}\text {-majority favour increase in } \\
\text { pay rise for health } \\
\text { personnel }\end{array}$ & \\
\hline 4 & $\begin{array}{l}\text { Macq Jean et al. } \\
\text { Human Res. for } \\
\text { Health in Deve. } \\
\text { Jour. 2001 }\end{array}$ & $\begin{array}{l}\text {-Reports on income } \\
\text { generation and work } \\
\text { mix among civil } \\
\text { servants in public } \\
\text { health services in } \\
\text { deve. countries }\end{array}$ & $\begin{array}{l}\text { Questionnaire } \\
\text { study }\end{array}$ & $\begin{array}{l}\text {-Respondents have median } \\
\text { salary of } 3800 \text { USD PPP } \\
\text { annually with fringe } \\
\text { benefits } \\
\text {-DP helps allows for a } \\
\text { standard of living that can } \\
\text { attract and keep doctors in } \\
\text { the public service } \\
\text { - source of motivation to } \\
\text { keep managing public } \\
\text { sector include } \\
\text { - social responsibility, self- } \\
\text { realization, } \\
\text { - time and conflict of } \\
\text { interest appear prominent } \\
\text { in this study } \\
\text {-Competition for time is } \\
\text { results in transfer of salary- } \\
\text { sources out of public sector } \\
\text { through reduced } \\
\text { availability- salary mass of } \\
27 \% \text { hidden out flow of } \\
\text { - hidfice } \\
\text { resources include office }\end{array}$ & $\begin{array}{l}\text {-use of mai } \\
\text { yielded a lo } \\
\text { rate } \\
\text {-rationale fo } \\
\text { size and } \\
\text { methodology } \\
\text { clear } \\
\text { - sample frar } \\
\text { MD not spec }\end{array}$ \\
\hline
\end{tabular}




\begin{tabular}{|c|c|c|c|c|c|}
\hline & & & & $\begin{array}{l}\text { infrastructure, public } \\
\text { sectors means of transport } \\
-\quad \text { and diagnostics and } \\
\text { therapeutics resources }\end{array}$ & \\
\hline 5 & $\begin{array}{l}\text { Chaud hury, et } \\
\text { al. } 2006 \\
\text { Jour of Econ } \\
\text { perspective }\end{array}$ & $\begin{array}{lr}\text {-Health } & \text { worker } \\
\text { absence } & \text { in } \\
\text { developing countries }\end{array}$ & $\begin{array}{l}\text { Questionnaire } \\
\text { based study }\end{array}$ & $\begin{array}{l}\text {-above } 40 \% \text { absence } \\
\text { among health workers in } \\
\text { India, Bangladesh 35\%, } \\
\text { Indonesia 40\%, Peru } 25 \\
\text { and Uganda 37\% }\end{array}$ & $\begin{array}{l}\text { - not able } \\
\text { whether } \\
\text { means engag } \\
\text { DP } \\
\text { - unannounce } \\
\text { the health fa } \\
\text { ethical } \\
\text { - reasons for } \\
\text { not verified } \\
\text { whether abs } \\
\text { officially aut }\end{array}$ \\
\hline
\end{tabular}




\begin{tabular}{|c|c|c|c|c|c|}
\hline 6. & $\begin{array}{l}\text { Humphrey et al. } \\
2004 \\
\text { Soc. sc. and } \\
\text { Medicine }\end{array}$ & $\begin{array}{l}\text {-Investigated the } \\
\text { reason for DP } \\
\text { among consultants in } \\
\text { the English NHS, } \\
\text { views about the two } \\
\text { systems and how } \\
\text { they deal with any } \\
\text { conflict of interests }\end{array}$ & $\begin{array}{l}\text { Qualitative } \\
\text { (interviews) }\end{array}$ & $\begin{array}{l}\text { - Money was responsible } \\
\text { for engaging in private } \\
\text { practice } \\
\text { - strategic influence - the } \\
\text { doctor is the king in private } \\
\text { practice - not under } \\
\text { bureaucratic administrative } \\
\text { rule } \\
\text {-Personal considerable } \\
\text { control over whom to treat } \\
\text { and how many to treat. } \\
\text {-Status and recognition } \\
\text { increase with private work } \\
\text {-professional opportunities } \\
\text { to practice new skills in the } \\
\text { private sector- cases that } \\
\text { may not come to the public } \\
\text { can be handled in the } \\
\text { private sector } \\
\text { Balancing NHS work and } \\
\text { private } \\
\text { - A few have not attempted } \\
\text { to leave NHS for fear of } \\
\text { losing private referral from } \\
\text { NHS colleagues }\end{array}$ & $\begin{array}{l}\text { - Revealed } \\
\text { seated reasor } \\
\text { - }\end{array}$ \\
\hline
\end{tabular}




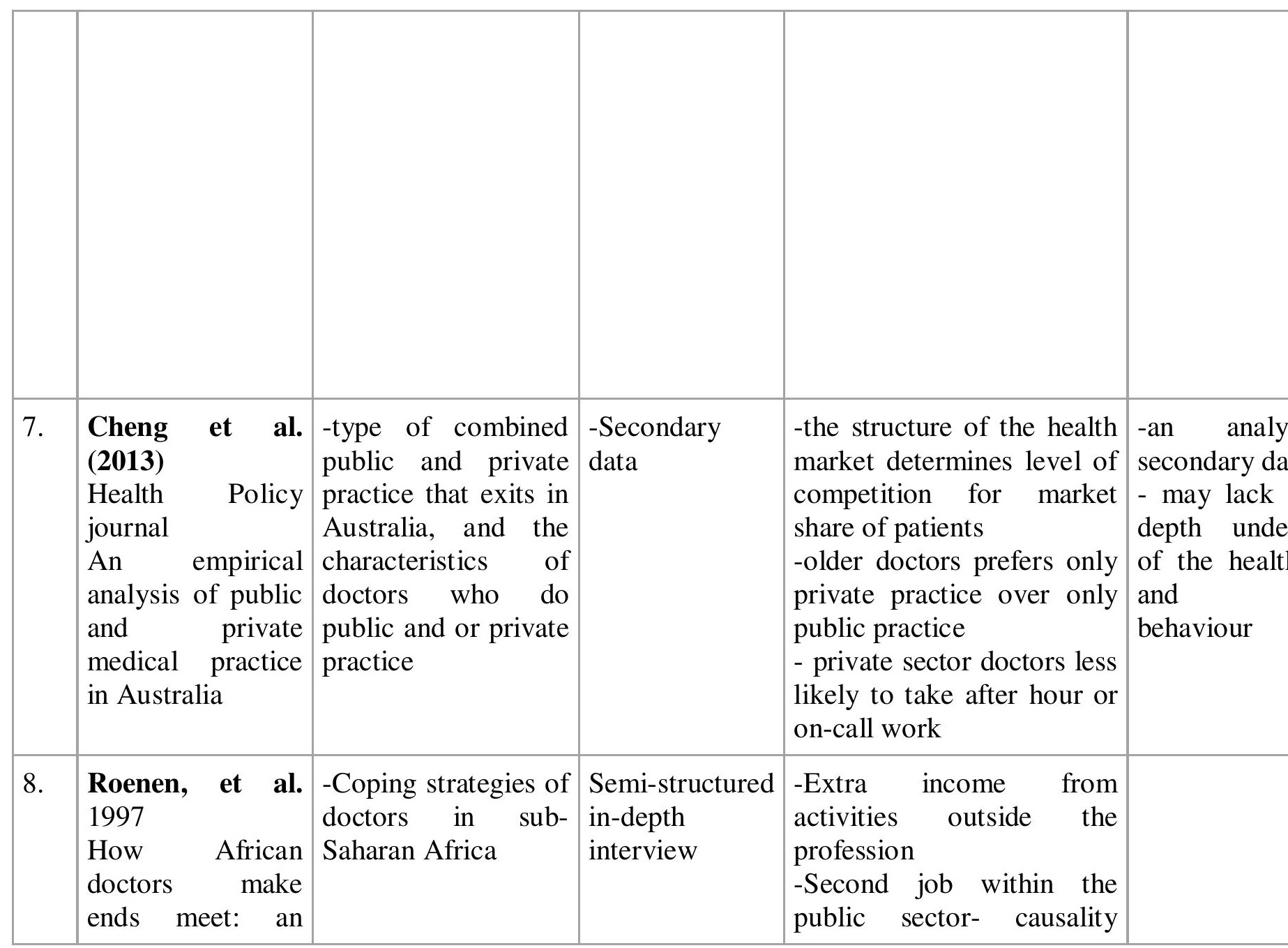




\begin{tabular}{|l|l|l|}
\hline exploration & $\mid \begin{array}{l}\text { shift }- \text { while this was not } \\
\text { possible in the past } \\
\text {-Allowances and per diem } \\
\text { as resource person } \\
\text {-Private practice and } \\
\text { selling drugs along private } \\
\text { practice } \\
\text {-presents and social support } \\
\text { from patients } \\
\text { the coping strategies } \\
\text { employed was purely in } \\
\text { search of decent living } \\
\text { standard }\end{array}$ \\
\hline
\end{tabular}

